\title{
Experimental investigation on repair and self-healing of tension-induced cracks in reinforced concrete panels
}

\author{
By: \\ Alireza Ahmadi \\ Bachelor of Science in Civil Engineering, \\ Shiraz University, Shiraz, Iran, 2016
}

\begin{abstract}
A Thesis
presented to Ryerson University

in partial fulfillment of the requirements for the degree of Master of Applied Science in the program of Civil Engineering
\end{abstract}

Toronto, Ontario, Canada, 2020

(C) Alireza Ahmadi 2020 


\section{AUTHOR'S DECLARATION}

I hereby declare that I am the sole author of this thesis. This is a true copy of the thesis, including any required final revisions, as accepted by my examiners.

I authorize Ryerson University to lend this thesis to other institutions or individuals for the purpose of scholarly research.

I further authorize Ryerson University to reproduce this thesis by photocopying or by other means, in total or in part, at the request of other institutions or individuals for the purpose of scholarly research.

I understand that my thesis may be made electronically available to the public. 


\title{
Experimental investigation on repair and self-healing of tension- induced cracks in reinforced concrete panels
}

\author{
Alireza Ahmadi \\ Master of Applied Science, 2020 \\ Department of Civil Engineering \\ Ryerson University, Toronto, Canada
}

\begin{abstract}
This research concentrates on repair and self-healing of tension cracks in RC panels subjected to direct tensile loading and hydrostatic water pressure by incorporating high-performance materials including High-strength epoxy, Glass Fiber Reinforced Polymer (GFRP) laminate, Engineered Cementitious Composite (ECC) slag, and ECC fly ash. The test setup simulates repairing internal surface of a cracked wall segment of a containment exposed to internal hydrostatic pressure. The study showed the applicability of ECC fly ash in repairing RC specimens with the ability to restore the structural strength along with reduced cracking, leakage, and enhanced self-healing behavior. GFRP exhibited high effectiveness in waterproofing and repair of damaged RC structures. Applicability of epoxy injection in local repair of cracked RC specimens was confirmed, while brittle failure of epoxy resulted in development of a large crack and high leakage rate. Moreover, GFRP was found to be the most economical technique for repair of RC containments.
\end{abstract}




\section{ACKNOWLEDGEMENTS}

The author would like to express his sincere gratitude to his supervisor Dr. Reza Kianoush for having provided this great research opportunity. Without his continuous guidance, suggestions, and encouragement this study would not have been accomplished. The author is also deeply grateful to the to the postdoctoral fellows of the Civil Engineering Department, Dr. Mehdi Moslemi and Dr. Hocine Siad, for their brilliant ideas and great help for casting concrete in the Structures and Concrete laboratories.

Thanks are extended to Mr. Nidal Jaalouk, and Mr. Bilal Baradie for their kind assistance in conducting the experimental tests.

Special thanks to my examining committee members, Dr. Khandaker M. A. Hossain, and Dr. Homayoun Abrishami for their helpful advice and suggestions.

Finally, I am deeply and forever indebted to my parents, for their love, encouragement and support throughout my entire life. 


\section{Dedication}

To my parents

who sacrificed their life for my greatest success 


\section{Table of Contents}

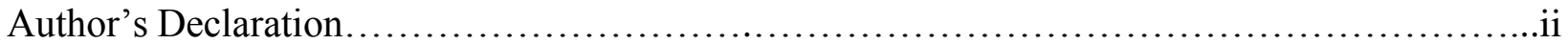

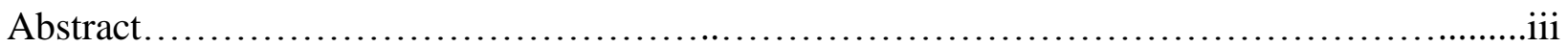

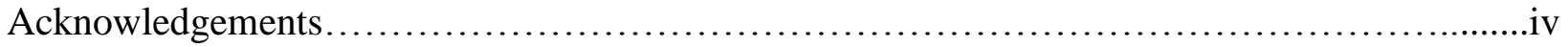

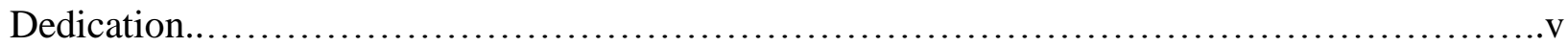

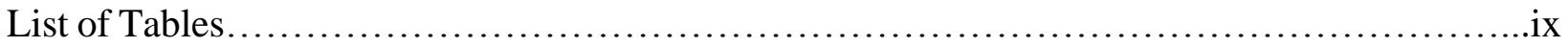

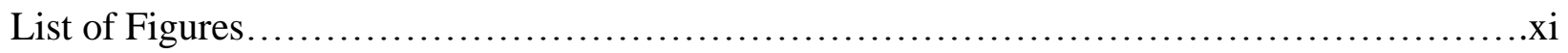

List of Symbols and Abbreviations........................................................

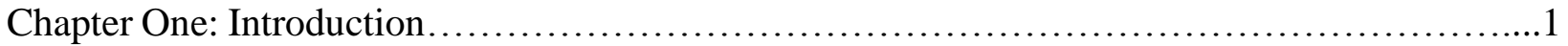

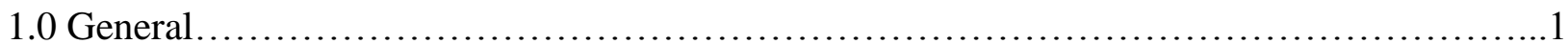

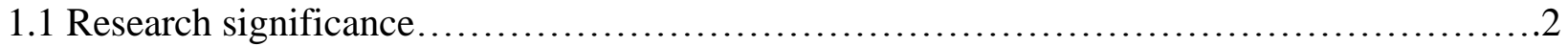

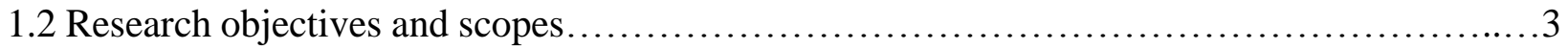

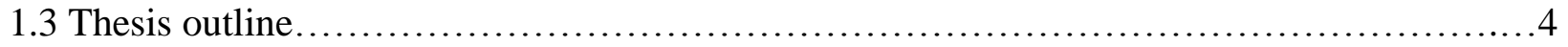

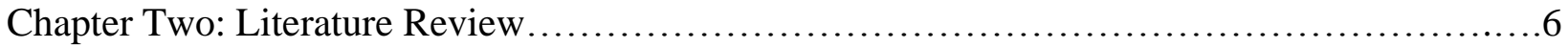

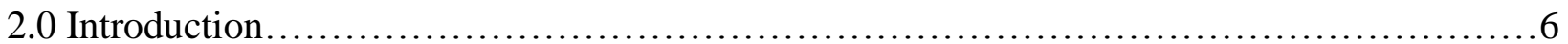

2.1 Engineered Cementitious Composite (ECC) mix design and material properties...............8

2.1.1 ECC mix design and mechanical properties.......................................... 8

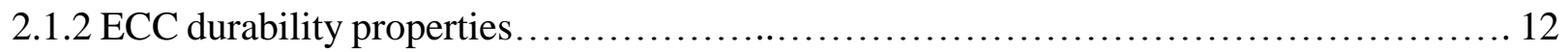

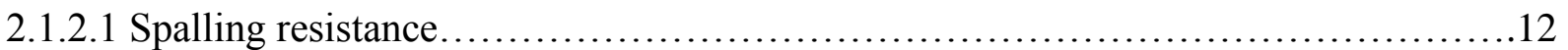

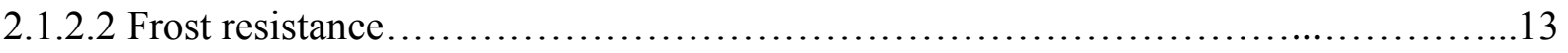

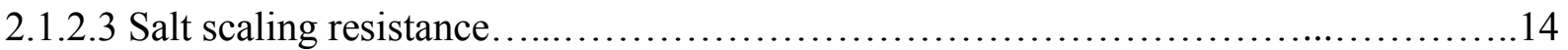

2.1.2.4 Durability under tropical climate exposure.......................................16

2.1.2.5 Alkali-silica reaction resistance................................................... 16

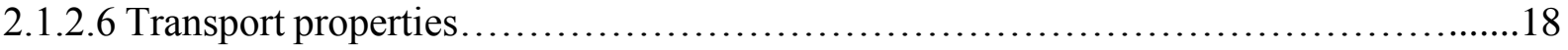

2.1.2.6.1 Water permeation........................................................... 19

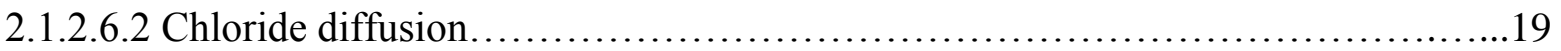




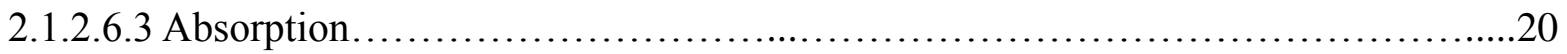

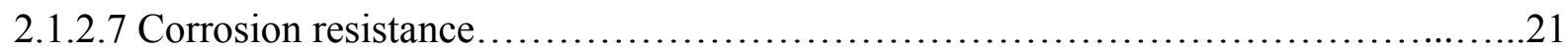

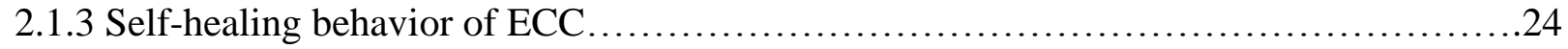

2.2 Application of ECC in construction and repair of full-scale structures ......................... 31

2.3 Various techniques of repair and strengthening of RC members.......................... 37

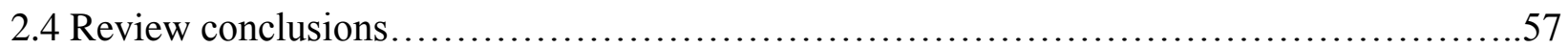

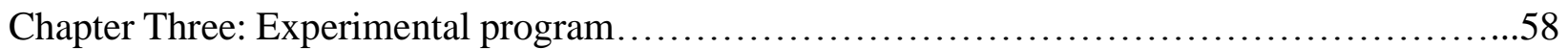

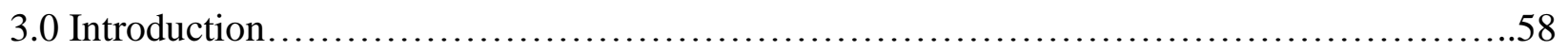

3.1 Geometric dimensions of specimens and reinforcement details.........................58

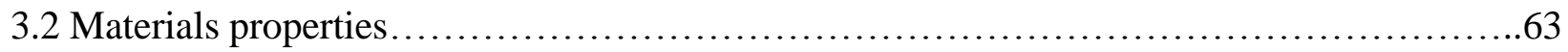

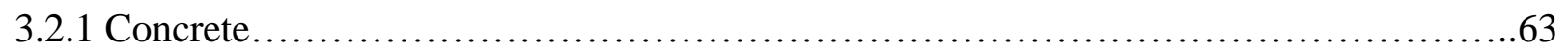

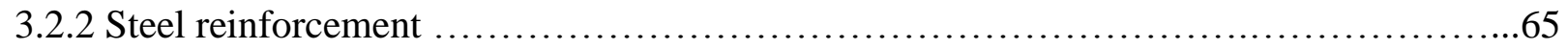

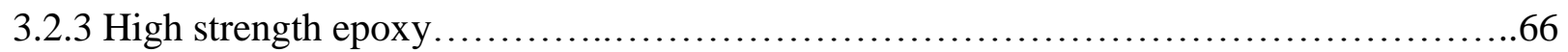

3.2.4 Glass Fiber Reinforced Polymer (GFRP) laminates.................................67

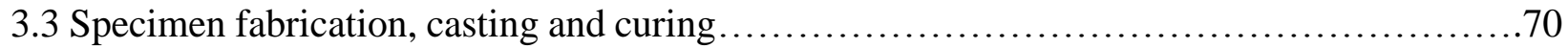

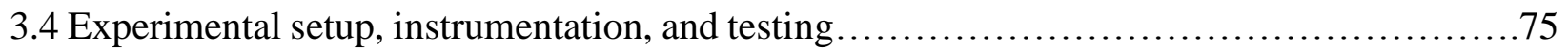

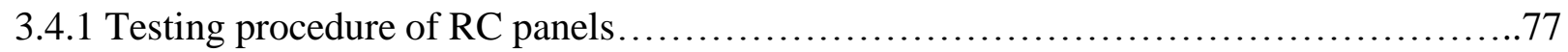

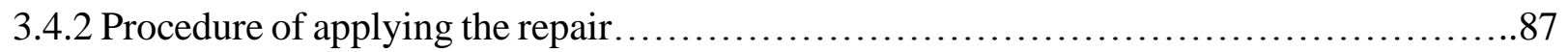

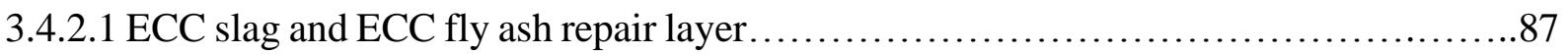

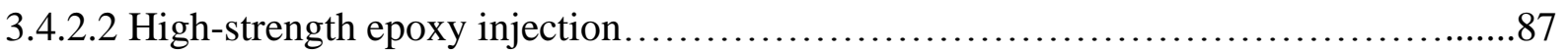

3.4.2.3 Glass Fiber Reinforced Polymer (GFRP) laminate.............................99

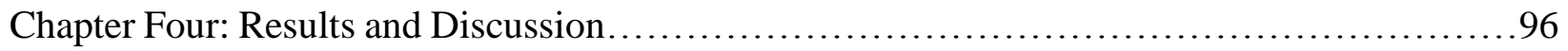

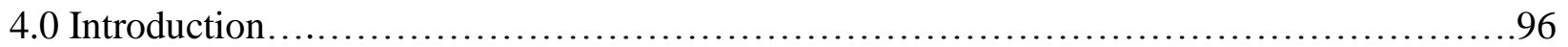

4.1 Performance of RC panels before repair............................................. 96

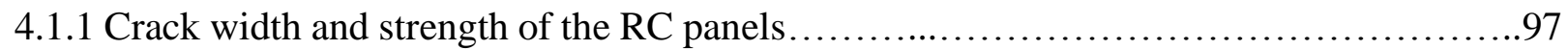

4.1.2 Strain in steel and load versus displacement in panels.................................98

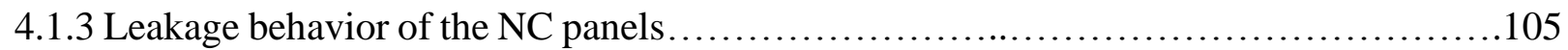


4.1.4 Self-healing of the NC panels.......................................................... 108

4.2 Performance of the repaired RC panels ............................................. 110

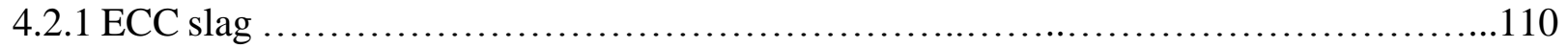

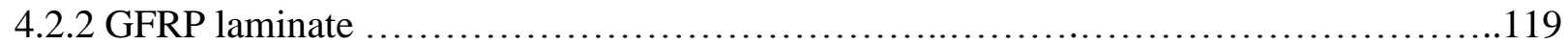

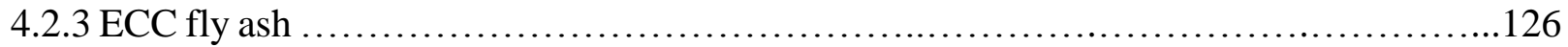

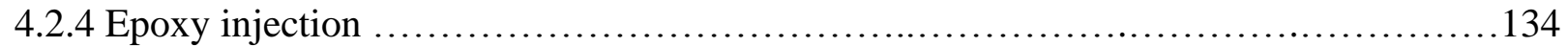

4.3 Comparison of the efficiency of different repair materials................................. 143

4.4 Comparison of the self-healing behavior before and after repair using ECC ..................147

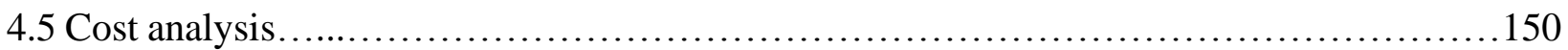

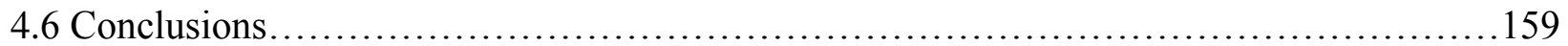

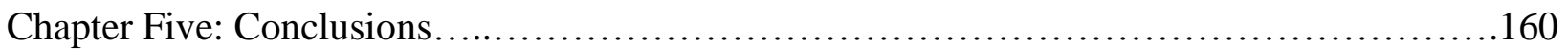

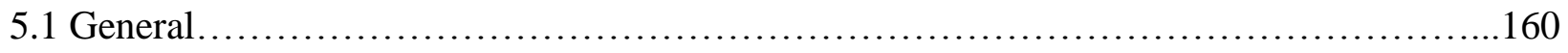

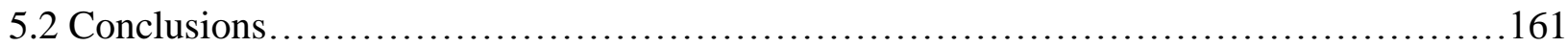

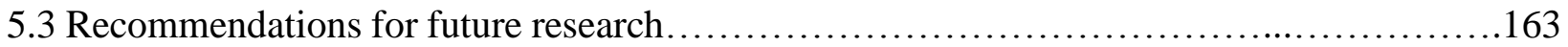

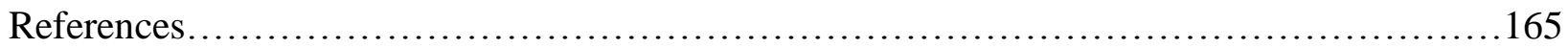




\section{List of Tables}

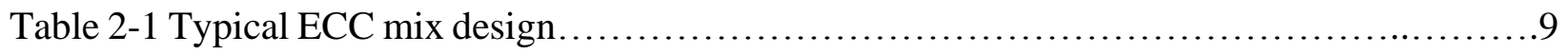

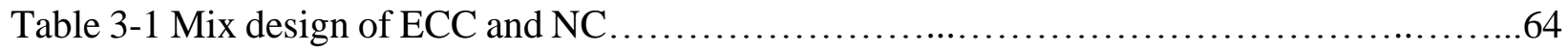

Table 3-2 Properties of steel reinforcement........................................65

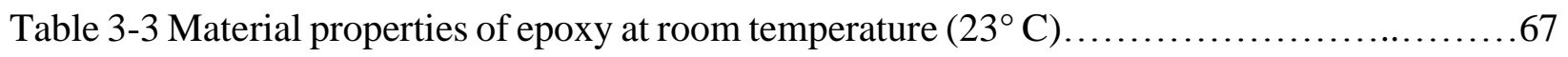

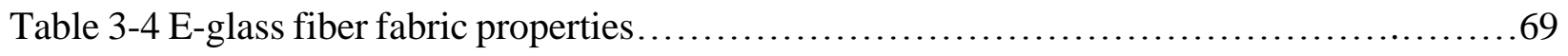

Table 3-5 Properties of the resin, hardener, and epoxy resin system.......................69

Table 3-6 Cured laminate properties after standard curing (7 days at 21 to $24^{\circ} \mathrm{C}$ )............69

Table 3-7 Concrete compressive, flexural, and tensile strength..........................75

Table 4-1 Load at leakage initiation and average cracks width of NC specimens...............98

Table 4-2 Leakage rate of the NC samples under different water pressures (before repair).......106

Table 4-3 Summary of the load, crack width, and leakage rate in NC panels..................108

Table 4-4 Tensile load and crack width in NC panel and ECC slag repair layer................111

Table 4-5 Performance of NC1 before and after repair using ECC slag.......................118

Table 4-6 Summary of the load and crack width of NC2 after repair using GFRP..............120

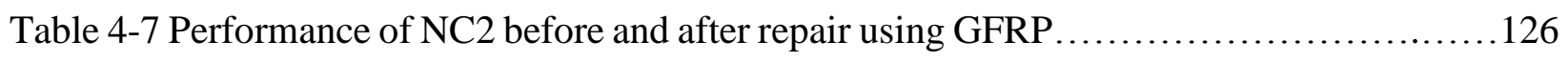

Table 4-8 Tensile load and crack width in NC panel and ECC fly ash repair layer..............128

Table 4-9 Performance of NC3 before and after repair using ECC fly ash....................133

Table 4-10 Summary of the load and crack width of NC4 after repair using epoxy.............135

Table 4-11 Summary of the tensile load, crack width, and leakage rate of the high-strength epoxy repaired NC4 panel under various water pressures............................................ 140

Table 4-12 Performance of NC4 before and after repair using high-strength epoxy.............143

Table 4-13 Summary of the performance of different repair materials.......................146 
Table 4-14 Calculation of repair cost for tank capacities between 10500 and 65000 Liter.......153

Table 4-15 Calculation of repair cost for tank capacities between 107000 and 260000 Liter.....154

Table 4-16 Calculation of repair cost for tank capacities between 315000 and 850000 Liter.....155

Table 4-17 Calculation of repair cost for tank capacities between 950000 and 4000000 Liter....156 


\section{List of Figures}

Figure 2-1 Typical uniaxial stress-strain curve and crack width development of ECC.............10

Figure 2-2 ECC beam behavior under flexural loading ................................. 11

Figure 2-3 Failure modes of ECC and NC slabs.......................................

Figure 2-4 ESEM micrograph of the self-healing products over the crack inside the ECC specimen after 30 days of exposure to the alkaline (sodium hydroxide) solution.......................... 18

Figure 2-5 Reinforced ECC and mortar samples after exposure to accelerated corrosion: (a): R/ECC prisms after 300 hours of accelerated corrosion, (b): R/mortar prisms after 75 hours of accelerated corrosion, (c): R/ECC cylinder samples after 350 hours of accelerated corrosion, (d): $\mathrm{R} /$ mortar samples after 95 hours of accelerated corrosion ................................. 24

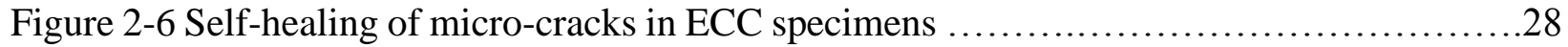

Figure 2-7 Self-healing products within the crack exposed to a) $20^{\circ} \mathrm{C}$ water b) $60{ }^{\circ} \mathrm{C}$ water c)

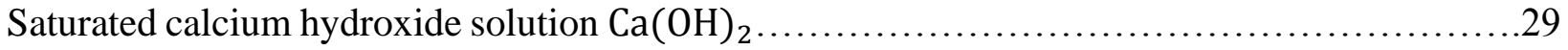

Figure 2-8 Deterioration due to Alkali-silica reaction (ASR) in a gravity retaining wall in Gifu,

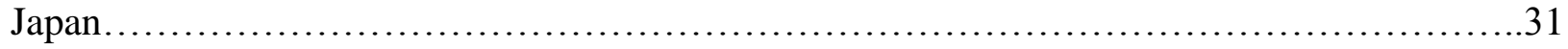

Figure 2-9 Repair of a gravity retaining wall using ECC (a) Condition of the wall after the repair program (b) Cracking behavior of the wall after the repair at 12 months (c) Cracking behavior of

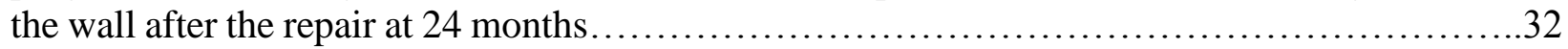

Figure 2-10 ECC repair application (a) Patch repair of bridge deck of the Curtis Road over M-14 in Southern Michigan (b) Crack width development versus time in ECC and concrete repair

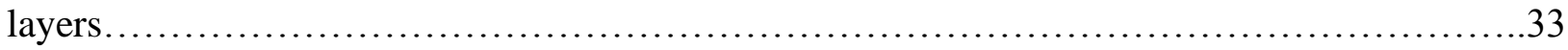

Figure 2-11 Mihara Bridge in Hokkaido, Japan ...................................... 34

Figure 2-12 Water-proofing of Mitaka Dam in Hiroshima-Prefecture, Japan in 2003 by spraying ECC repair layer................................................................ 34

Figure 2-13 ECC link slab of Grove Street Bridge, Michigan, USA a) ECC casting b) Finished

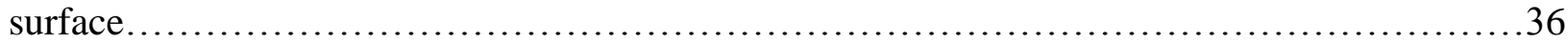

Figure 2-14 Schematic of using ECC coupling beams (in yellow) in high-rise buildings.........37

Figure 2-15 Premature failure due to CFRP strip peeling .................................

Figure 2-16 Failure of the beams repaired with epoxy a) Failure of the type 1 epoxy at original cracked section b) Failure of the type 2 and type 3 epoxies at different location from original fracture line 
Figure 2-17 Failure of the beam-column joint under cyclic loading a) Failure occurance at the joint before repair b) Failure occurance outside of the joint after repair with epoxy injection..........43

Figure 2-18 Failure of the bam-column joint under cyclic loading a) Failure occurance at the joint before repair b) Failure occurance outside of the strengthened area after repair with epoxy

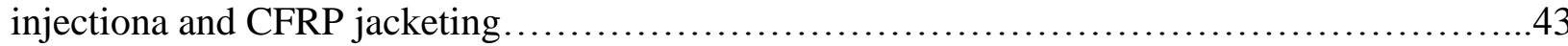

Figure 2-19 Repair of RC beams using low-pressure epoxy injection a) Sealing the cracked surface and installing the injection ports b) Low-pressure epoxy injection.......................... 45

Figure 2-20 Failure of the beam-column joint under lateral cyclic loading a) Formation of plastic hinge at the joint location b) Formation of the plastic hinge in the beam member away from the

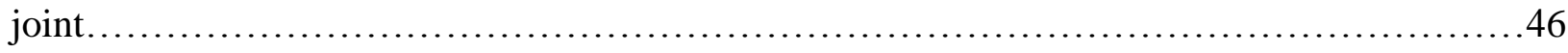

Figure 2-21 Strengthening with ECC at plastic hinge region............................. 47

Figure 2-22 Strengthening with ECC layer on the tension side............................48

Figure 2-23 Failure mode of the strengthened RC beams with BFRP grid and ECC a) BFRP tensile rupture and concrete crushing b) BFRP tensile rupture and debonding of the strengthening layer. .50

Figure 2-24 Cross-section of the strengthened RC beams with $40 \mathrm{~mm}$ thick U-shape ECC

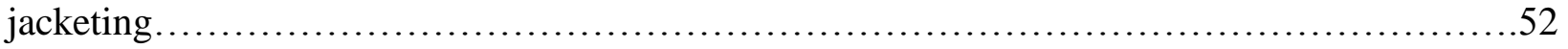

Figure 2-25 Strengthening methods of RC beam specimens with ECC layer in flexure............53

Figure 2-26 Column strengthening with ECC and BFRP textile a) Basalt fiber mesh textile b) Confined column details...........................................................55

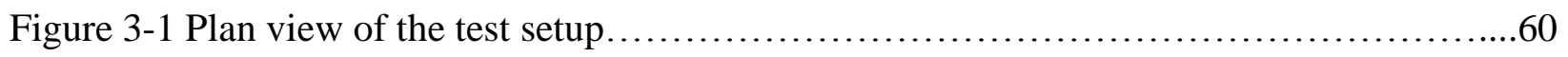

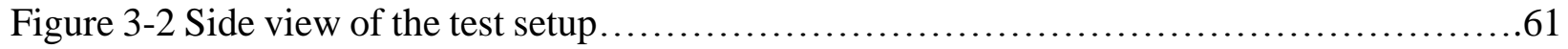

Figure 3-3 Details of the water pressure chamber.....................................62

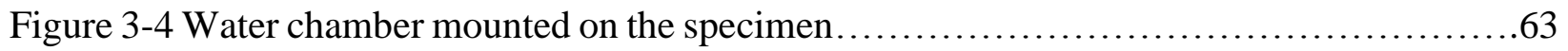

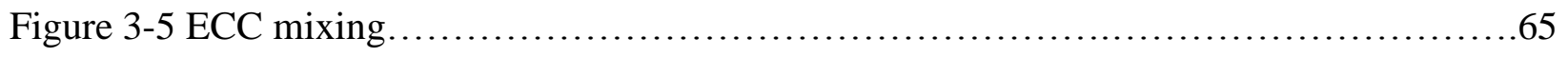

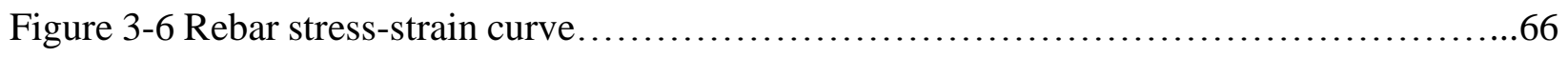

Figure 3-7 Low viscosity of the high-strength epoxy ................................67

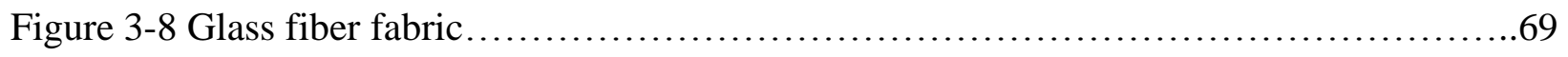

Figure 3-9 Typical RC panel form-work ........................................69

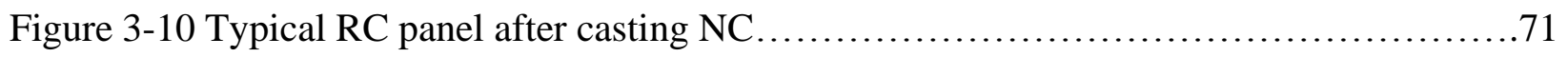


Figure 3-11 Moist curing of the RC panels after casting with NC

Figure 3-12 Repair layer formwork on top of the RC panel..............................73

Figure 3-13 ECC repair layer after casting with control samples..........................74

Figure 3-14 Hardened cylinder specimens after grinding a) ECC- fly ash b) NC c) ECC-slag......75

Figure $3-15$ Actual side view of the specimen........................................ 76

Figure 3-16 Arrangement of steel strain gauges on the rebar..............................77

Figure $3-17$ plan view of the specimen and loading test setup $\ldots \ldots \ldots \ldots \ldots \ldots \ldots \ldots \ldots \ldots \ldots . \ldots \ldots$

Figure 3-18 Water pressure chamber mounted on NC panel.............................78

Figure 3-19 Crack formation in NC panel under direct tension a) Bottom face b) Side face........79

Figure 3-20 Extreme leakage through a major crack in one of the NC panels (before repair)......80

Figure 3-21 Figure 3-21: Releasing the tensile load and applying the repair a) NC specimen after releasing the tensile load and removing the water chamber and rubber pad b) NC specimen after applying a $50 \mathrm{~mm}$ ECC repair layer.................................................... 81

Figure 3-22 NC specimen repaired with a) ECC overlay b) GFRP laminates...................82

Figure 3-23 Top and bottom surfaces of a NC specimen repaired with high-strength epoxy injection at mid-span crack a) Bottom surface b) Top surface $\ldots \ldots \ldots \ldots \ldots \ldots \ldots \ldots \ldots \ldots \ldots \ldots . \ldots \ldots 2$

Figure 3-24 Repaired NC specimen in the re-load test setup $\ldots \ldots \ldots \ldots \ldots \ldots \ldots \ldots \ldots \ldots \ldots \ldots . \ldots \ldots$

Figure 3-25 NC specimen repaired with a $50 \mathrm{~mm}$ layer of ECC slag.........................85

Figure 3-26 NC specimen repaired with a $50 \mathrm{~mm}$ layer of ECC fly ash......................85

Figure 3-27 NC specimen repaired with a) GFRP laminates b) High-strength epoxy injection......86

Figure 3-28 Procedure of drilling the NC panel and removing the dust using vacuum cleaner and

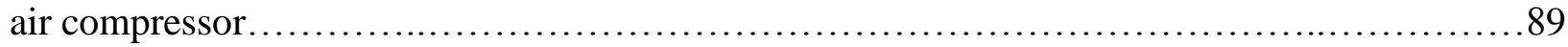

Figure 3-29 Installation of the injection ports on top of the holes and sealing the bottom surface of

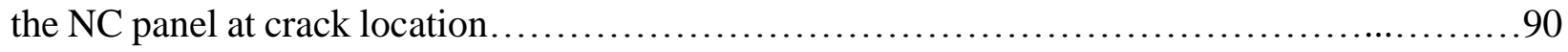

Figure 3-30 High-pressure epoxy injection..........................................

Figure 3-31 Finished surfaces of the NC panel after high-pressure epoxy injection a) Bottom

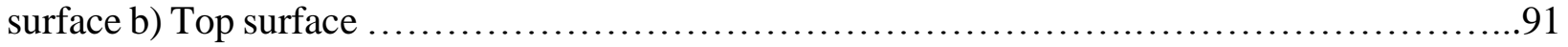

Figure 3-32 Prepared surface of the RC panel for applying GFRP $\ldots \ldots \ldots \ldots \ldots \ldots \ldots \ldots \ldots \ldots . . . \ldots 3$ 
Figure 3-33 Mixing the resin and hardener and applying the epoxy resin system on the top surface

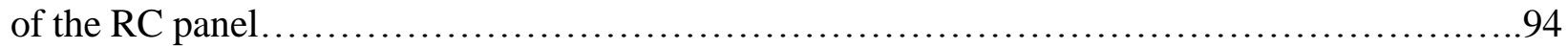

Figure 3-34 Finished surface of the RC after applying the GFRP ..........................95

Figure 4-1 NC specimen cracked at mid-span a) Bottom face b) Side face......................98

Figure 4-2 Arrangement of steel strain gauges on the rebar................................ 99

Figure 4-3 Steel strain development at typical strain gauge in a) NC1 b) NC2 c) NC3 d) NC4

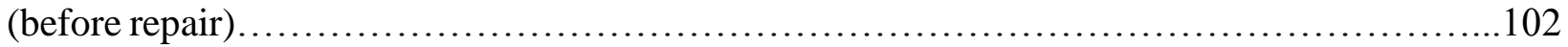

Figure 4-4 Location of LVDT at the middle of the specimen................................103

Figure 4-5 Load- relative horizontal displacement at mid-span of a) NC1 b) NC2 c) NC3 d) NC4

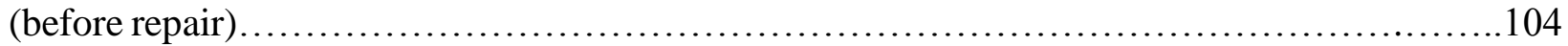

Figure 4-6 Leakage rate of the NC panels under different water pressures.....................105

Figure 4-7 Extensive leakage in NC specimens at crack initiation (before repair) ...............107

Figure 4-8 Short-term self-healing of NC2 sample (before repair).........................109

Figure 4-9 Long-term self-healing of NC2 sample (before repair).........................110

Figure 4-10 Leakage behavior of NC1 before and after repair using ECC slag..................112

Figure 4-11 Leakage of the NC1 specimen repaired using ECC slag under 8 psi water pressure

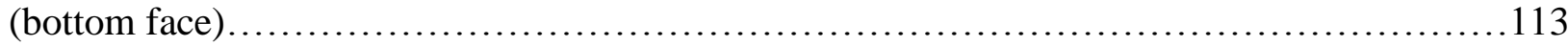

Figure 4-12 Tensile steel strain development in NC1 repaired using ECC slag.................114

Figure 4-13 Tensile load versus relative horizontal displacement of the repaired NC1 panel with

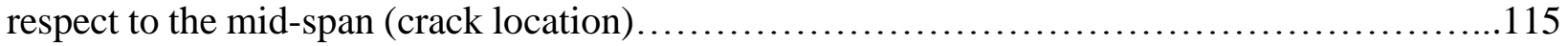

Figure 4-14 Short-term self-healing of the NC1 repaired using ECC slag.....................116

Figure 4-15 Long-term self-healing of the NC1 repaired using ECC slag......................117

Figure 4-16 Leakage of the NC panel before and after repair using ECC slag a) Before repair b)

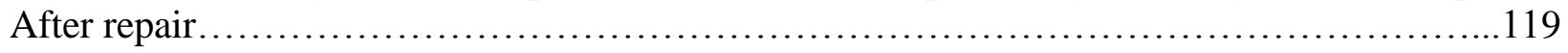

Figure 4-17 Tensile load versus crack width of the NC2 specimen repaired using GFRP

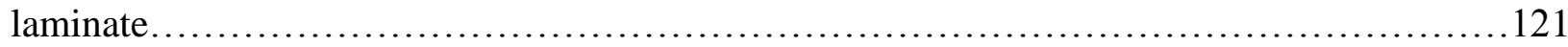

Figure 4-18 Mid-span crack width in NC2 panel before and after repair with GFRP.............123

Figure 4-19 Tensile strain development in the rebar in GFRP repaired NC2 panel...............124 
Figure 4-20 Tensile load versus horizontal displacement of the GFRP repaired NC2 specimen with

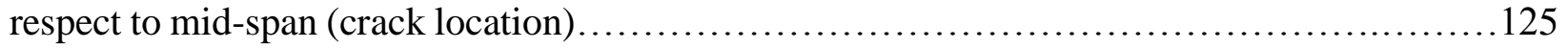

Figure 4-21 Leakage behavior of NC3 before and after repair using ECC fly ash.................128

Figure 4-22 Leakage of the NC3 specimen repaired using ECC fly ash under 8 psi water pressure

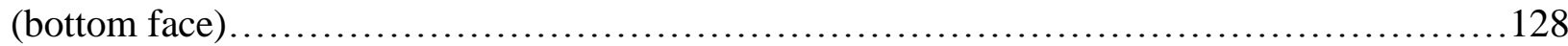

Figure 4-23 Tensile steel strain development in NC3 repaired using ECC fly ash................129

Figure 4-24 Tensile load versus relative horizontal displacement of the NC3 panel with respect to

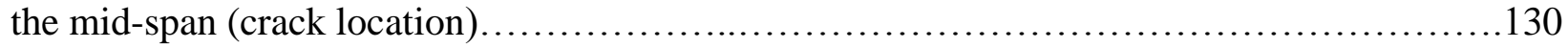

Figure 4-25 Short-term self-healing of NC3 panel repaired using ECC fly ash..................131

Figure 4-26 Long-term self-healing of NC3 panel repaired using ECC fly ash...................132

Figure 4-27 Leakage of the NC panel before and after repair using ECC fly ash a) Before repair b)

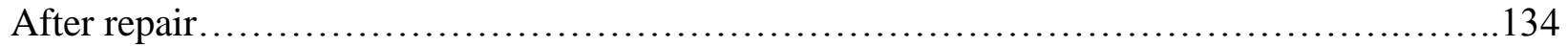

Figure 4-28 Tensile load and crack width of the NC4 specimen repaired using epoxy...........136

Figure 4-29 Comparison of the leakage behavior of NC4 before and after repair using epoxy...138

Figure 4-30 Brittle failure of the epoxy at crack location (mid-span) and high leakage rate.......138

Figure 4-31 Tensile steel strain development in NC4 panel repaired using high-strength epoxy..141

Figure 4-32 Tensile load versus relative horizontal displacement of the NC4 panel with respect to

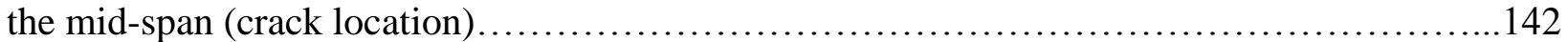

Figure 4-33 Comparison of the self-healing behavior of the NC panel before and after repair using ECC slag and ECC fly ash.............................................................

Figure 4-34 Different shapes of the tanks a) Circular b) Rectangular c) Spherical d) Iznte e) Circular with conical bottom......................................................... 150

Figure 4-35 Classification of tank based on placement condition a) Resting on the ground b)

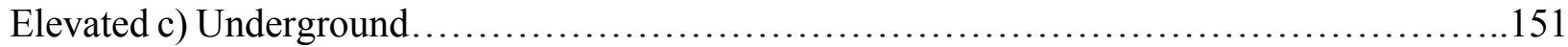

Figure 4-36 Total repair cost for commercial and industrial tanks.......................... 157

Figure 4-37 Total repair cost for residential tanks.................................... 158 


\section{List of Symbols and Abbreviations}

RC: Reinforced concrete

NC: Normal concrete

ECC: Engineered cementitious composite

HPFRCC: High-performance fiber reinforced cementitious composite

GFRP: Glass fiber reinforced polymer

CFRP: Carbon fiber reinforced polymer

BFRP: Basalt fiber reinforced polymer

$f_{c}^{\prime}$ : Cylinder compressive strength of concrete

$f_{t}$ : Cylinder tensile strength of concrete

$f_{y}$ : Yielding strength of the steel rebar

$\varepsilon_{s}:$ Strain in the rebar

$\varepsilon_{y}:$ Yielding strain

$A_{s}:$ Area of the reinforcement

$\rho_{s}:$ Steel reinforcement ratio

$d_{b}$ : Diameter of the rebar

B: Width of concrete cross section

$\mathrm{H}$ : Depth of the concrete cross section

SCMs: Supplementary cementitious materials

LCS: Liquid containing structure

$\mathrm{C}_{2} \mathrm{~S}$ : Dicalcium silicate

$\mathrm{C}_{3} \mathrm{~S}$ : Tricalcium silicate

CSH: Calcium silicate hydrate

$\mathrm{Ca}(\mathrm{OH})_{2}$ : Calcium hydroxide

$\mathrm{OH}^{-}$: Hydroxide ions

$\mathrm{Ca}^{2+}:$ Calcium ions

$\mathrm{SiO}_{2}$ : Silicone dioxide

$\mathrm{CO}_{3}^{2-}$ : Carbonates

$\mathrm{HCO}_{3}^{-}$: Bio-carbonates 
$\mathrm{CaCO}_{3}$ : Calcium carbonate

ESEM: Environmental scanning electron microscope

RF: Resonant frequency

CRC: Conventionally reinforced concrete

MDOT: Michigan department of transportation

PP: Poly propylene

PVA: Polyvinyl alcohol

FA/C: Fly ash/cement ratio

ASR: Alkali silica reaction

FRCM: Fiber reinforced cementitious mortar

TRM: Textile reinforced mortar

SHCC: Strain hardening cementitious composite

HSRS: High-strength reinforcing steel

GGBS: Ground granulated blast furnace slag

HRWR: High range water reducer 


\section{Chapter One: Introduction}

\section{1-0 General}

During the 20s century, most of the civil infrastructure in industrialized countries have been built using normal concrete (NC). However, due to low tensile strength and high permeability of NC, civil infrastructures have been experiencing various modes of deterioration during the last decades. In most cases, partial or complete demolition and reconstruction of the deteriorated structure is neither economical, environmental friendly, nor practical. Therefore, the need for repair and rehabilitation seems to be inevitable. Reinforced concrete members usually crack due to structural loads or restrained shrinkage and thermal deformations which are usually inevitable in practice (Wittmann, 2002; Mihashi and De Leite, 2004). Crack formation is identified as the starting point of the deterioration in reinforced concrete structures. Concrete cracking is a major concern in structures that are designed under serviceability limit states including liquid containing structures. Prevention or control of the crack width is of prime importance in such structures as it causes leakage from the container as well as providing a pathway for aggressive agents to penetrate to the concrete, causing corrosion of reinforcement and other forms of deterioration, which affects both functionality and durability of the liquid containing structures.

Tremendous progress in concrete industry during the last decades resulted in the development of a class of concrete materials capable of strain hardening behavior as High-Performance Fiber Reinforced Cementitious Composites (HPFRCC), as a fundamental solution for durability problems corresponding to the cracking and brittle nature of $\mathrm{NC}$. By considering the need for applicability, constructability, and cost-effectiveness, and by optimizing the mix design, 
Engineered Cementitious Composites (ECC) was introduced as a ductile HPFRCC with special characteristics including strain hardening, high tensile strain capacity, multiple cracking behavior, and high self-healing ability (Li, 1993; Li, 2019).

$\mathrm{NC}$ is characterized by low tensile strength along with brittle nature, while ECC is commonly known as high ductile material, with tight crack width and moderate tensile strength from $2 \mathrm{MPa}$ to $6 \mathrm{MPa}(\mathrm{Li}, 1993$; Fischer et al., 2003). ECC exhibits tensile strain capacity in the range of 3-5\% through continuous development of closely spaced microcracks. Such a multiple cracking behavior in ECC results in strain capacity 300 to 500 times higher than NC. After formation of these microcracks, ECC is still capable of increasingly carrying the load resulting in strain hardening behavior similar to many ductile metals. The most distinctive characteristics separating ECC from NC and other types of HPFRCC are high ductility under uniaxial tensile loading in the range of 3$7 \%$ along with tight crack width of around $60-100 \mu \mathrm{m}$, with a relatively low fiber content of $2 \%$ by volume, resulting in reducing the permeability and improving the durability properties (Wang and Li 2007; Sahmaran and Li 2009; Sahmaran et al. 2011; Li, 2019).

Superior mechanical and durability properties make ECC an ideal choice for both construction and repair of RC structures, offering a fundamental solution to overcome durability problems associated with cracking and brittle nature of NC. Use of ECC results in facilitating the casting process, due to its self-compacting capacity, as well as improving the fresh-states, mechanical, and durability properties of RC structures.

\section{1-1 Research significance}

Extensive research has been performed on material properties of ECC for tailoring improved mechanical and durability properties. ECC has also been used for several real-world structural 
applications over the years. A number of investigations have been conducted on the application of ECC in repair and strengthening of RC members under various loading conditions. However, there is no research available in the literature focusing on the repair of liquid containing structures using ECC. This study is warranted and will investigate the applicability of ECC in repairing liquid containing structures by evaluating the ECC performance in repairing NC panels subjected to direct tensile loading under hydrostatic water pressure. Moreover, ECC efficiency is compared to currently used repair materials in industry including high-strength epoxy and FRP laminates in terms of strength, cracking and leakage behavior, and self-healing ability. The findings of this research can be directly applicable to structures that are designed under serviceability limit states including liquid containing structures, in which crack control, water tightness and leakage behavior are of prime importance. The outcomes and recommendations of this research are expected to benefit engineers, builders, and local authorities when constructing and repairing liquid containing structures.

\section{1-2 Research objectives and scopes}

The main objectives of this research program are to:

- Study the cracking and leakage behavior of normal concrete (NC) panels under direct tensile loading which simulates the wall segment of a tank under an internal hydrostatic pressure. Analyze the performance based on the load at which leakage initiated, crack width, as well as leakage rate under different water pressures corresponding to the various water heights in the tank.

- Compare the efficiency of different repair materials in terms of strength, cracking and leakage behavior under direct tensile loading. Materials used in the repair program include a low-viscosity 
high-strength epoxy, Glass Fiber Reinforced Polymer (GFRP) laminates, ECC using slag, and ECC using fly ash.

- Study the self-healing of the tension cracks in NC panels before and after repair with ECC slag and ECC fly ash.

\section{1-3 Thesis outline}

The thesis consists of 5 chapters including experimental investigation on repair and self-healing of tension induced cracks in RC panels by incorporating high-performance materials including high-strength low viscosity epoxy, GFRP laminates, ECC slag, and ECC fly ash.

Chapter 1 presents general remarks about deterioration of RC structures and need for repair, followed by research significance and objectives of this study.

Chapter 2 presents a comprehensive literature review on various techniques of repair and strengthening of RC members concentrating on epoxy injection, FRP laminates, ECC, and combined application of ECC with FRP grids. Moreover, research performed on structural applications of reinforced ECC (R/ECC) members is presented. Literature on materials, mix design and properties of ECC is also reviewed. It also presents research conducted on self-healing behavior of ECC.

Chapter 3 describes the experimental program by detailing the material properties and geometrical dimensions of the concrete panels, casting and curing of samples, test-setup, instrumentation and testing procedure.

Chapter 4 reports the results of the experimental investigations on the repair of full-scale NC panels under direct tensile loading. The performance of ECC slag, ECC fly ash, high-strength 
epoxy, and GFRP laminates in repairing NC panels exposed to direct tensile loading under hydrostatic pressure is described based on experimental results. Load versus horizontal displacement responses, strain development in the steel rebar, crack development and crack width, and leakage rate under various water pressures are analyzed to evaluate the performance of each repair material in terms of tensile load capacity, cracking and leakage behavior. Self-healing behavior of the concrete materials is also investigated.

Chapter 5 points out the main findings of the research program and makes suggestion for future research. 


\section{Chapter Two: Literature Review}

\section{2-0 Introduction}

Reinforced concrete deterioration and its durability are two correlated concepts. Durability of a reinforced concrete $(\mathrm{RC})$ structure is a major concern and largely depends on how dense the microstructure is. A dense concrete mixture leads to higher strength and lower permeability and also reduces the rebars corrosion rate through limiting the ingress of the aggressive agents to the concrete. This can be achieved by proper selection of the concrete mix ingredients including addition of fibers and use of low water to binder (W/B) ratio (Mehta, 1986), well graded aggregates (Hwang et al.,1996), and supplementary cementitious materials such as silica fume, slag, and fly ash (Chang et al., 2001). Reinforced concrete members usually crack due to structural loads or restrained shrinkage and thermal deformations which are usually inevitable in practice (Wittmann, 2002; Mihashi and De Leite, 2004). Crack formation which threatens the durability of reinforced concrete structures is of prime importance. These cracks provide the pathway for aggressive agents including chloride, oxygen, and carbonation agents to penetrate to the concrete and cause reinforcement corrosion and other forms of deterioration. The main cause of concrete cracking and consequently durability problems is the low tensile strength of the normal concrete (NC) and its brittle nature. Therefore, a fundamental solution is required to overcome the problem of brittle nature of the NC. By incorporating the new generation of High-Performance Fiber Reinforced Cementitious Composites (HPFRCC) which exhibits superior fresh-state, mechanical, and durability properties including high tensile ductility and strain hardening behavior, problems regarding concrete deterioration resulted from cracking can be solved (Li and Stang, 2004). 
Addition of fibers to the concrete is not a new phenomenon and dates back to the Egyptian times by adding straws or horsehair to mud bricks. Moreover, straw mats were also used as the reinforcement in the housing construction by Japanese and Chinese in the past. Later on, numerous attempts were made to develop HPFRCC materials with desirable properties by adding continuous aligned fibers (Aveston et al., 1971; Krenchel \& Stang, 1989), and discontinuous fibers (Allen, 1971; Lankard, 1986; Naaman, 1992) for achieving high levels of ductility. A class of concrete materials capable of strain hardening behavior was eventually developed as High-Performance Fiber Reinforced Cementitious Composites (HPFRCC). By considering the need for applicability, constructability, and cost-effectiveness, and by optimizing the mix design, Engineered Cementitious Composites (ECC) was introduced as a ductile HPFRCC with special characteristics including strain hardening, high tensile capacity, multiple cracking behavior, and self-healing ability (Li, 1993; Chu, 2014; Li, 2019). Such a superior performance makes ECC an appropriate choice for both construction and repair of RC structures. Use of ECC results in facilitating the casting process, due to its self-compacting capacity, as well as improving the fresh-states, mechanical, and durability properties of RC structures.

The chapter presents the following: (a) mix design and material properties of ECC (b) practical applications of ECC in construction and repair of RC structures, (c) research studies conducted on the repair and strengthening of RC members using ECC, FRP laminates, and epoxy injection, (d) self-healing behavior of ECC (e) summary to illustrate that the proposed experimental program is needed to contribute to the existing knowledge of the technology. 


\section{2-1 ECC mix design and material properties}

\section{2-1-1 ECC mix design and mechanical properties}

ECC which was originally invented at the University of Michigan in the early 1990s ( $\mathrm{Li} 1993 ; \mathrm{Li}$, 2019), was introduced as a solution to the brittle nature of NC and as a material exhibiting multiple micro-cracking behavior, high tensile ductility and strain hardening behavior along with selfhealing ability (Nawy, 2008). The most distinctive characteristics separating ECC from NC and other types of HPFRCC is high ductility under uniaxial tensile loading in the range of 3-7\% along with tight crack width of around 60-100 $\mu \mathrm{m}$, with a relatively low fiber content of $2 \%$ by volume, resulting in reducing the permeability and improving the durability properties (Wang and Li, 2007; Sahmaran and Li, 2009; Sahmaran et al., 2011; Li, 2019). Moreover, ECC exhibits tensile strain capacity in the range of 3-5\% through continuous development of closely spaced microcracks. Such a multiple cracking behavior in ECC results in strain capacity 300 to 500 times higher than NC. After formation of these microcracks, ECC still can increasingly carry the load resulting in strain hardening behavior similar to many ductile metals. Special characteristics of ECC such as strain hardening, multiple cracking behavior, and high ductility are achieved through micromechanical tailoring of the ingredients including cementitious materials, aggregates, and fibers (Li, 1998; Lin et al., 1999; Li et al., 2001; Li, 2003; Li, 2019). The typical ECC mix components are cement, fine aggregates, fibers, water, and supplementary cementitious materials as a partial replacement of the cement such as fly ash and slag. Fibers are added to the ECC mix to improve the tensile strength, ductility, toughness, and durability. The typical fibers which are usually used in ECC mix are polypropylene (PP), glass (GF), carbon (CF), and polyvinyl alcohol (PVA) (Cavdar, 2012). The most common used fiber in ECC mix is Polyvinyl alcohol (PVA) with 
a length of 6-12 mm and diameter of $39 \mu \mathrm{m}$ (Li et al. 2001; Kunieda and Rokugo 2006). Performance of the fibers in the ECC mix depends on the mix properties as well as fiber properties including fiber type, size, volume, and dispersion, along with the interfacial properties between the mix and fibers. In order to maintain adequate flowability and workability of the ECC mix, while keeping the water to cementitious materials ratio as low as 0.25 to 0.3 , a High Range Water Reducer (HRWR) is usually used in ECC mix. A typical ECC mix is presented in Table 2-1.

Table 2-1: Typical ECC mix design (Sahmaran and Li, 2009)

\begin{tabular}{|c|c|}
\hline Material & Proportion \\
\hline Cement & 1 \\
\hline Water & 0.54 \\
\hline Fly ash & 1.2 \\
\hline Fine aggregates & 0.54 \\
\hline Fiber (Vol.) $\%$ & 2 \\
\hline Superplasticizer & 0.013 \\
\hline
\end{tabular}

One major difference between the mix design of ECC and conventional concrete is the elimination of the coarse aggregates and consequently incorporating higher cement content. Higher cement content helps to improve fiber dispersion, workability and flowability, and strain hardening behavior. Due to economical and environmental purposes, as well as improving the mix properties, supplementary cementitious materials (SCMs), which are usually by-products of other industries such as fly ash and slag, are used in ECC mix as a partial replacement of the cement. In case of aggregates, silica sand (with average particle size of 100-200 $\mu \mathrm{m}$ ), crush sand or gravel sand (with average particle size of 1 to $2 \mathrm{~mm}$ ) are used. Presence of coarse aggregates in the mix may negatively affect the performance of the fiber reinforcement through hindrance of microscale 
interaction between the fibers and the mix. Moreover, low matrix fracture toughness is also improved by elimination of coarse aggregates (Lepech et al., 2008).

Strain hardening, defined as the ability of the mix to keep carrying the load even after the formation of the first crack, and usually found in ductile metals such as steel, can be achieved in ECC by incorporating of relatively low content of $2 \%$ by volume of PVA fiber. Figure 2-1 illustrates a typical uniaxial tensile stress-strain curve with high strain capacity of around 5\%. ECC shows high tensile ductility, strain hardening behavior, as well as strain capacity up to several hundred times of normal concrete. Crack width in ECC remains below $80 \mu \mathrm{m}$ even at ultimate load. Micro cracking behavior of the ECC is independent of the type of loading and fiber content (Özbay et al., 2013; Yeganeh, 2013; Li, 2019).

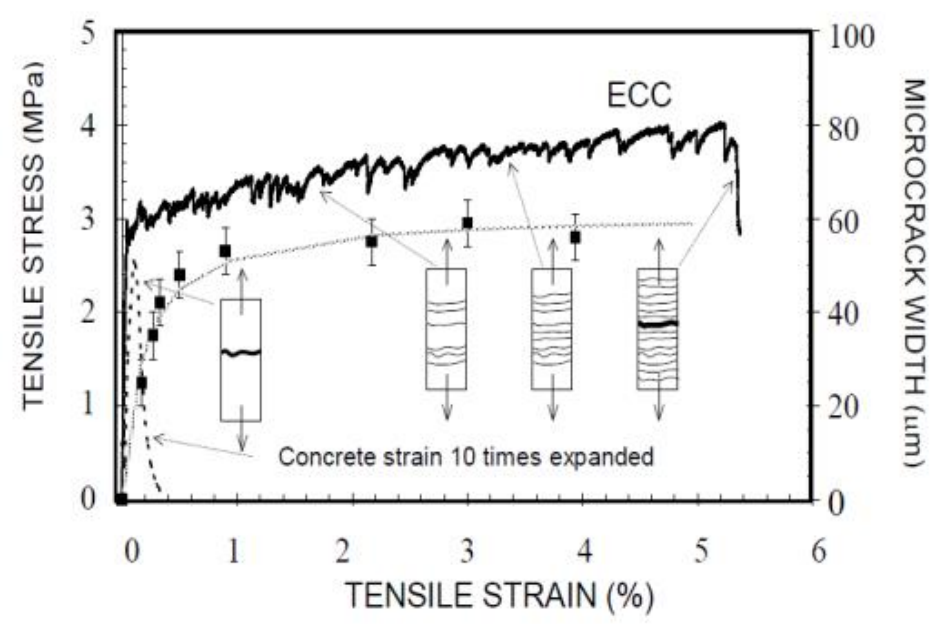

(a)

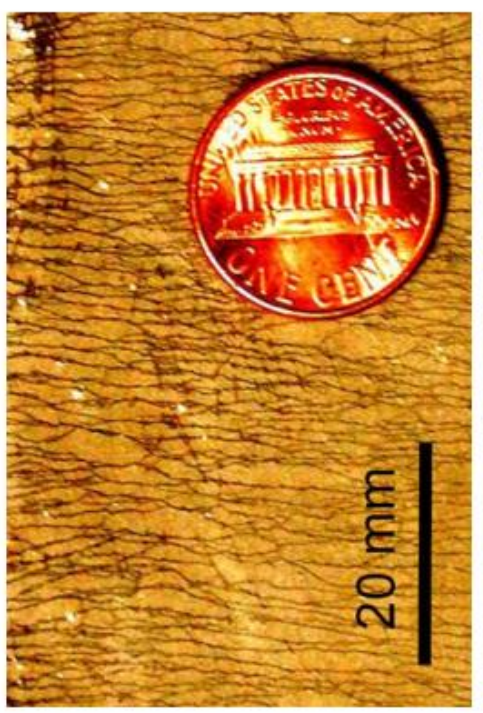

(b)

Figure 2-1: ECC mechanical properties a) Typical uniaxial stress-strain curve b) Crack width development (Weimann and $\mathrm{Li}, 2003$ )

Under flexural loading, the ECC beam behaves the same as a ductile metal in its plastic range of deformation, which is shown in Figure 2-2. Moreover, ECC behavior in compression is similar to a high strength concrete reaching around $60 \mathrm{MPa}$ (Lepech and Li, 2008; Li, 2019). 
Mechanical properties of ECC were investigated by Şahmaran et al. (2009) by incorporating different aggregate types (with different maximum aggregate size), and fly ash/cement ratios. In this experimental program, different types of aggregate including micro-silica sand (lowest maximum size of aggregate), dolomite limestone crushed sand (intermediate maximum size of

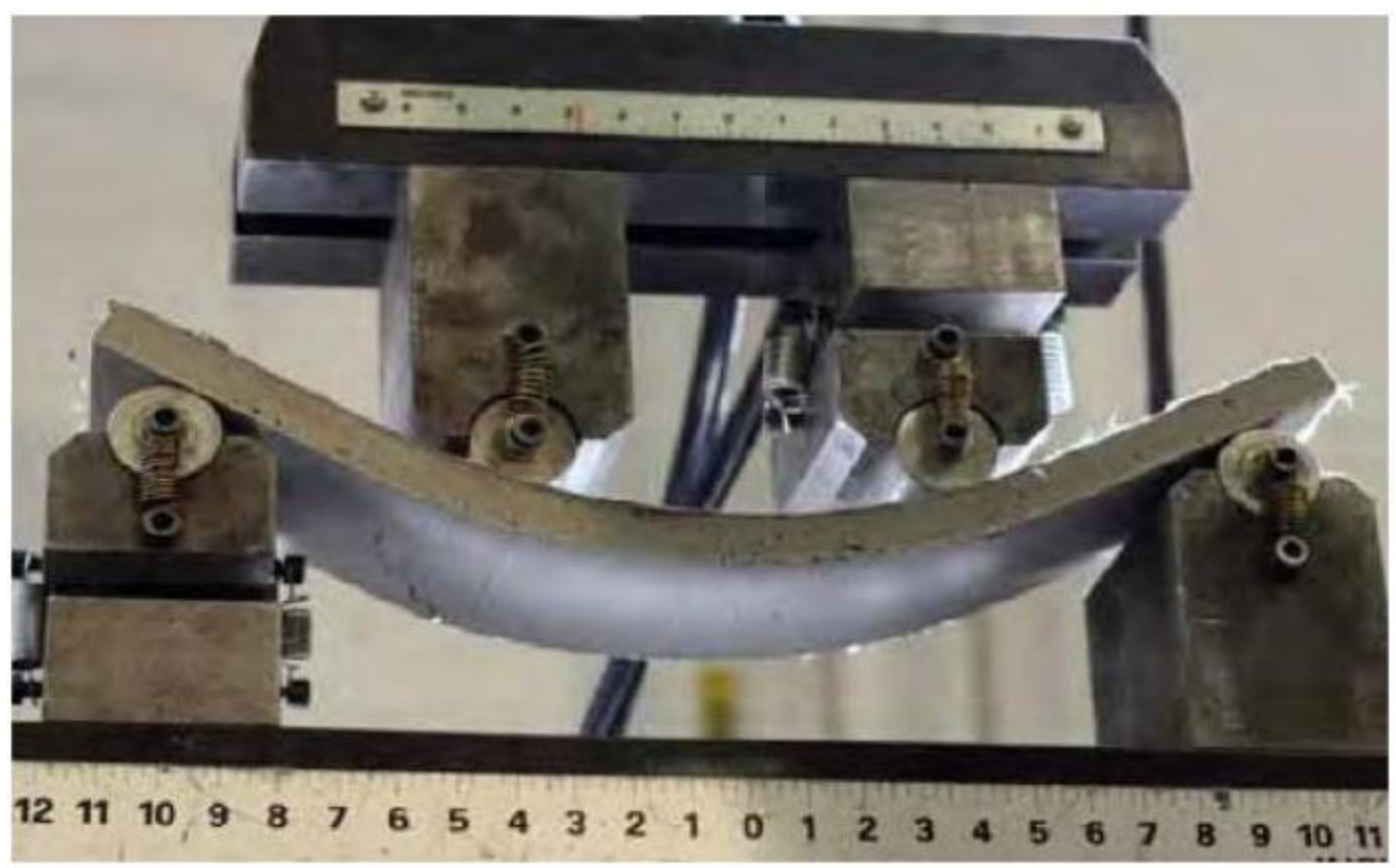

Figure 2-2: ECC beam behavior under flexural loading (Li, 2011)

aggregate), and gravel sand (highest maximum size of aggregate) were used. Also, fly ash/cement (FA/C) ratio as another variable was changing from 1.2 to 4.2 in this study. Based on the compressive strength test results, increasing fly ash percentage reduced the compressive strength of the ECC. Moreover, incorporating micro-silica resulted in higher compressive strength compared to crushed sand and gravel sand. However, it was concluded by Şahmaran et al. (2009) that unlike NC, aggregates surface texture and aggregate size does not significantly affect the compressive strength of ECC. Moreover, it was observed that by increasing the FA/C ratio, the tensile strength regarding formation of first crack decreased. However, increasing the fly ash 
content, enhanced the tensile strain capacity (Wang \& Li, 2007). It was found by Şahmaran et al. (2009) that enhancement of fly ash content reduced the crack width in ECC, while aggregate size did not affect the ECC crack width significantly. Moreover, under flexural loading microcracks developed at midspan of ECC samples on the tension face propagating outward. ECC samples with FA/C ratio of 1.2 reached flexural strength up to $12.75 \mathrm{MPa}$, while by increasing the FA content, flexural strength decreased. Using gravel sand (highest maximum size of aggregates) reduced the ductility of the ECC samples that can be explained by negative effects of higher maximum size aggregates on the fiber dispersion and interfacial properties between the fibers and the mix.

Fresh state, mechanical, and durability properties of ECC have been the subject matters of intense research over the years and resulted in development of greener and cost-effective ECC by incorporating locally available crushed sand/ mortar sand aggregates instead of micro-silica sand and use of supplementary cementitious materials including fly ash/slag/metakaolin/volcanic ash as a partial replacement of cement (Sahmaran et al., 2009; Mavani, 2012; Ozbay et al., 2012; Sherir, 2012; Hossain and Anwar, 2014; Sherir et al., 2014; Sherir et al., 2015). Although each material affected the fresh state, mechanical, and durability properties of ECC, but similar responses were achieved based on experimental results.

\section{2-1-2 ECC durability properties}

\section{2-1-2-1 Spalling resistance}

Spalling resistance is an important durability property of the reinforced concrete structures. Among different modes of deterioration in RC members, reinforcement corrosion is defined by degradation of the rebar due to an electrochemical reaction with its environment. When the rebar 
corrodes, the rust forms on top of the rebar occupies a bigger volume causing a tensile circumferential stress state (hoop stress) on the concrete cover. More rebar corrosion and rust formation results in applying higher tensile stress on the concrete cover and eventually exceeding the tensile strength of the concrete which leads to cracking, propagation of the crack, and concrete spalling. Kanda et al. (2003) and Miyazato and Hiraishi (2005) investigated the spalling resistance by pushing a tapered steel into a hole in ECC and NC slabs. Effort has been made to simulate the tensile circumferential stress on the concrete generated by corroded rebar (rebar + rust). Based on the results, ECC experienced a ductile failure mode by formation of radial micro-cracks, while NC slabs failed in brittle nature by formation and propagation of one or several major cracks. At the same compressive strength level, ECC slabs tolerated significantly higher loads $(30 \mathrm{kN})$ compared to NC sample (7 kN). Failure modes of ECC and NC slabs are illustrated in Figure 2-3.

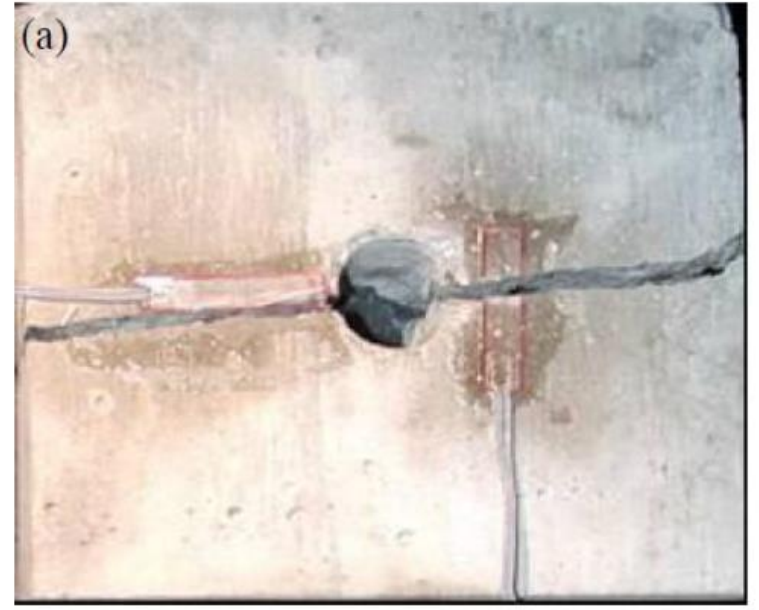

a) $\mathrm{NC}$

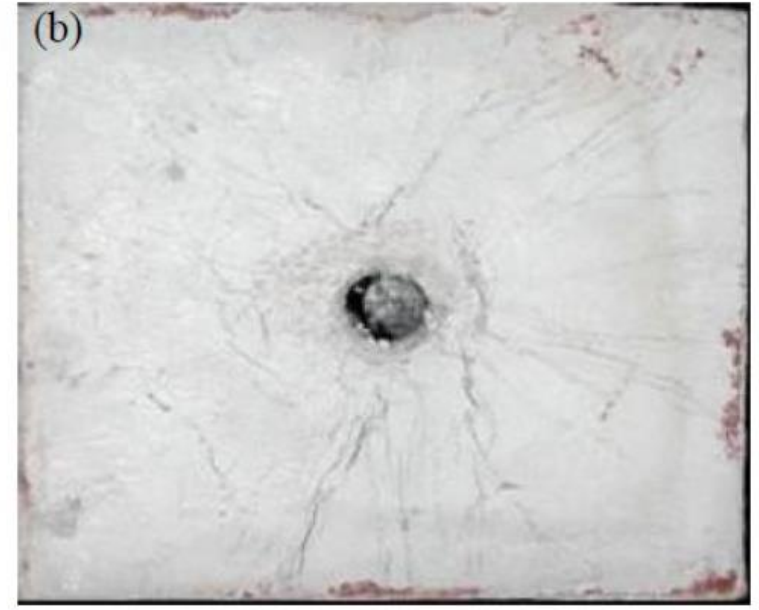

b) ECC

Figure 2-3: Failure modes of the specimens a) NC b) ECC

(Sahmaran and Li, 2010; Mavani, 2012)

\section{2-1-2-2 Frost resistance}

Another important durability property of the concrete is frost resistance, which is defined by the concrete ability to tolerate repeated cycles of freezing and thawing. Transition of water from liquid 
to ice results in increasing its volume by $9 \%$. Those pores inside the concrete, which are filled with water, will apply tensile stress to the concrete as a result of this volume increase which may cause cracking and eventually concrete spalling. In general, an air-entraining system is introduced as a solution for those normal concrete structures exposed to the freezing and thawing cycles including concrete pavements, bridge decks, and parking. Such an intentionally created air system inside the concrete provides a sufficient volume of pores, not filled with water, to accommodate water expansion during the freezing and thawing cycles. Li et al., (2003) investigated the performance of non- air entrained ECC samples under repeated cycles of freezing and thawing in accordance with ASTM C666 (2008). The experimental program was conducted on non-air-entrained NC and ECC prisms over the period of 14 weeks with 300 cycles. NC samples experienced severe deterioration during the first 5 weeks (110 cycles) and based on the testing standard were removed from the freezing and thawing machine. However, all ECC samples experienced no degradation of dynamic modulus after 300 cycles. According to ASTM C666 (2008), a durability factor of 100 was assigned to ECC compared to 10 to NC (Li, 2008; Mavani, 2012; Li 2019). Li et al., 2003 performed uniaxial tensile tests on the ECC samples after exposure to 300 cycles of freezing and thawing and was compared to the wet cured ECC samples at the same age. The results showed no significant decrease in strain capacity of the freeze thaw exposed and wet cured samples, both exhibiting strain capacity of 3\%. Moreover, it was found by Yun and Rokugo (2012) that after 300 freezing and thawing cycles, ECC retained $97 \%$ of the initial dynamic modulus (Li, 2008; Li, 2019).

\section{2-1-2-3 Salt scaling resistance}

Supplementary cementitious materials (SCMs) including slag and fly ash are widely used as a partial replacement of cement due to their economical, and environmental benefits. It has been 
indicated by previous test data that concrete samples made with high volumes of fly ash are more susceptible to cracking and spalling when exposed to freezing and thawing cycles in presence of de-icing salts. As in ECC mix design, around two third of Portland cement is replaced with fly ash $\mathrm{F}$, its performance should be evaluated in terms of exposure to freeze thaw cycle in presence of de-icing salts. Sahmaran and Li (2007) investigated the performance of non- air-entrained sound (uncracked) and mechanically preloaded (cracked) ECC samples during 50 cycles of freezing and thawing combined with de-icing salts and compared to non-air-entrained specimens according to ASTM C672 (2003). After exposure of the sample to 50 freeze thaw cycles in presence of de-icing salt, surface condition visual rating was performed and total mass of the scaling residue was calculated. ECC samples even with high fly ash content exhibited high durability by remaining within the acceptable limits of ASTM C672 (2003). The same satisfactory behavior was observed for cracked ECC samples even up to high deformations. In contrast, severe deterioration was observed in concrete samples in the same testing condition. Furthermore, increasing the fly ash content resulted in higher levels of deterioration in concrete samples in exposure to freeze thaw cycles in presence of de-icing salts. Sahmaran and Li (2007) also evaluated the tensile strength and ductility of the sound (uncracked) and preloaded (cracked) ECC samples after exposure to 25 and 50 cycles of freezing and thawing combined with de-icing salts. Based on the results, negligible loss of ductility was observed in preloaded samples. Preloaded samples also exhibited multiple cracking behavior as well as high strain capacity of more than 3\%. It was found by Sahmaran and $\mathrm{Li}$ (2007) that micro-cracks formed on the mechanically preloading stage of the ECC samples were healed during the exposure to the freezes thaw cycles in presence of salt, retaining the initial stiffness of the ECC samples. Results of this experimental program also confirm high levels of durability in both virgin (uncracked) and preloaded (cracked) ECC specimens in spite of exposure 
to repeated cycles of freezing and thawing in presence of de-icing salts (Li, 2008; Mavani, 2012; Li, 2019).

\section{2-1-2-4 Durability under tropical climate exposure}

In addition to investigating the ECC behavior in winter conditions with repeated freeze thaw cycles, it is important to evaluate the ECC performance in long-term exposure to hot and humid conditions. Li et al, 2004 performed hot water immersion on individual fibers, single fibers embedded in ECC matrix, and composite ECC material specimens to simulate the exposure to hot and humid conditions. Specimens were cured for 28 days in room temperature followed by immersion in hot water at $60^{\circ} \mathrm{C}$ for up to 26 weeks. After 26 weeks of immersion in hot water, pull out test was performed and Li et al, 2004 observed small change in fiber properties including strength, elastic modulus, and elongation. ECC samples experienced a reduction in tensile strain capacity from $4.5 \%$ (at early age) to $2.75 \%$, which is still 250 greater than normal concrete tensile strain capacity and is within the acceptable range for most civil infrastructure application, after 26 weeks immersion in hot water, equivalent to 70 years of natural weathering (Li, 2008; Mavani, 2012; Li, 2019).

\section{2-1-2-5 Alkali-silica reaction resistance}

Reinforced concrete structures are sometimes exposed to high alkaline environments that may affect the microstructure and composite properties through alkali-silica reactions (ASR). ECC performance was evaluated in terms of exposure to alkali- silica reaction by Sahmaran and Li (2008). This experimental program included measuring the length change of the ECC bar specimens after immersion in alkali solution at $80{ }^{\circ} \mathrm{C}$ for 30 days according to ASTM C1260 (1994). Fly ash C and fly ash F were used as a partial replacement of Portland cement in ECC mix. 
Based on the test results, ECC samples showed no significant expansion due to 30 days immersion in alkali solution. This can be explained by use of high-volumes of fly ash, which reduces the $\mathrm{pH}$ through the pozzolanic reaction resulting in less probability of alkali-silica reaction, as well as presence of PVA fibers in the ECC mix, which decreases the expansion resulted from ASR. Moreover, increasing the fly ash to cement ratio (FA/C) from 1.2 to 2.2 caused a reduction in length change in ECC samples (Li, V.C., 2008). Moreover, mechanical properties of virgin (uncracked) and preloaded (cracked) ECC sample were investigated by Sahmaran and Li, 2008 in exposure to high alkaline environments. The test included preloading of ECC specimens up to different strain levels, followed by immersion in the alkaline solution at $38{ }^{\circ} \mathrm{C}$ for 3 months and reloading up to the failure of the samples. Based on the results, preloaded (cracked) ECC samples exhibited small reduction in tensile strength and ductility, while capable of showing multiple micro-cracking behavior and retaining tensile strain capacity of more than $2 \%$, which is almost 200 times greater than normal concrete tensile strain capacity. It was also found by Sahmaran and Li, 2008 that self-healing of micro-cracks resulted in restoring the initial stiffness and ability of keep carrying the tensile stress and strain. An environmental scanning electron microscope (ESEM) observation of the self-healed crack inside the ECC specimen is shown in Figure 2-4, which confirms self- healing of crack after one month of exposure to the alkaline (sodium hydroxide) solution (Mavani, 2012; Li, 2019). 


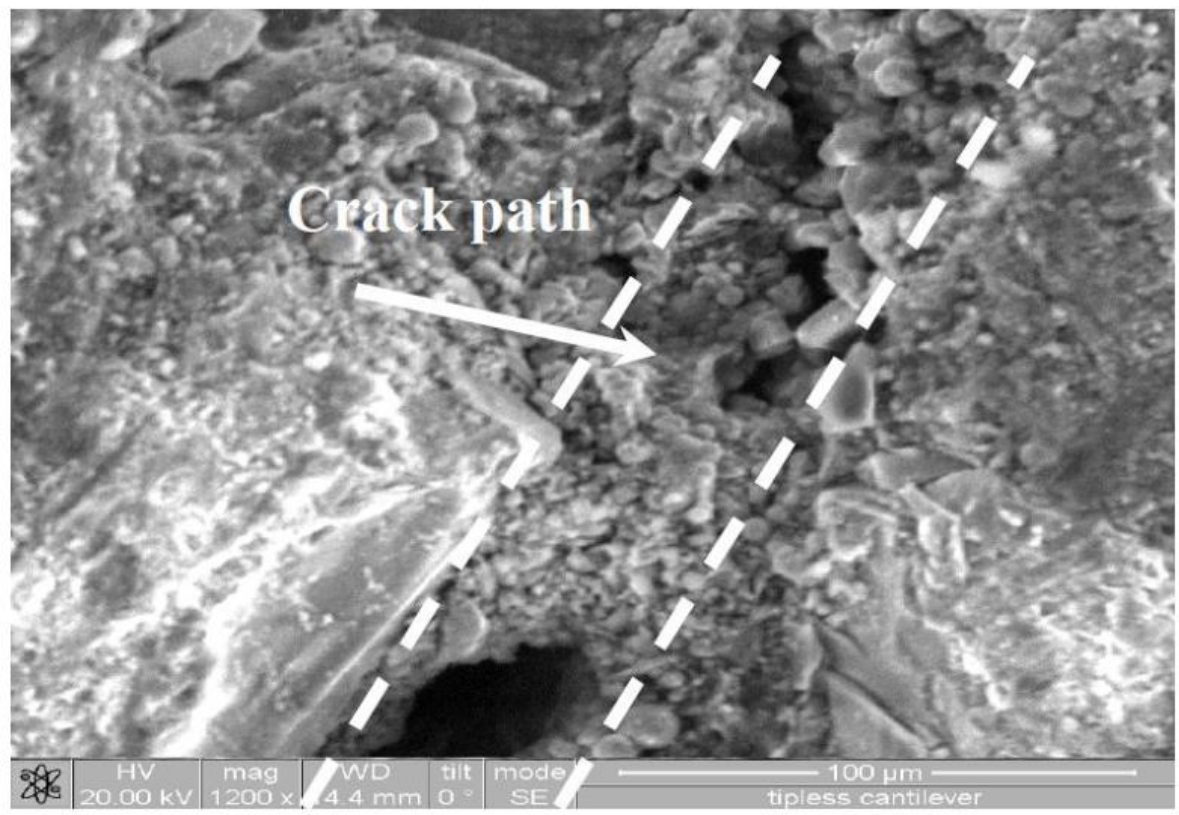

Figure2-4: ESEM micrograph of the self-healing products over the crack inside the ECC specimen after 30 days of exposure to the alkaline (sodium hydroxide) solution (Sahmaran et al., 2007; Mavani, 2012)

\section{2-1-2-6 Transport properties}

Reinforced concrete structures often experience cracking either intentionally to reach full tensile capacity of the rebars, or due to excessive structural loading, and restrained shrinkage or thermal deformations. Presence of cracks provides the opportunity for the liquids, gases, and ions to penetrate through the concrete causing various modes of deterioration. Transportation of these agents to the concrete mainly occur through three main mechanisms including permeation, diffusion, and capillary suction. Permeation which is an important phenomenon in liquid containing structures and reservoir dams is governed by presence of a hydraulic pressure (Lepech and Li, 2005a; Lepech and Li, 2006; Sahmaran and Li, 2008). Capillary pore suction is the governing mechanism when the unsaturated concrete is exposed to liquid. Diffusion occurs when there is an ion concentration gradient between the concrete surface and the ion solution such as chloride ion, which may happen in bridge decks, salt tanks and marine environments. 


\section{2-1-2-6-1 Water permeation}

Water permeability of the mechanically loaded ECC and reinforced mortars was investigated by Lepech and Li (2005a). For this purpose, specimens were loaded up to $1.5 \%$ tensile deformation causing different crack patterns along with various crack widths and number of cracks for ECC and reinforced mortar samples. Then, water permeability of the specimens was measured by applying a hydraulic head and calculating the coefficient of permeability. It was observed by Lepech and $\mathrm{Li}$ (2005a) that ECC samples exhibited micro-cracking behavior with maximum crack width of $60 \mu \mathrm{m}$, regardless of applied level of tensile deformation, while reinforced mortars experienced crack widths larger than $150 \mu \mathrm{m}$ under same loading. Reinforced mortars showed significantly higher water permeability than ECC samples due to development of larger cracks.

Interestingly, ECC exhibited same water permeability $\left(\mathrm{k} \sim 5 \times 10^{-11} \mathrm{~m} / \mathrm{s}\right)$ as the sound (uncracked) mortars.

\section{2-1-2-6-2 Chloride diffusion}

Rebar corrosion is an important mode of deterioration threatening the durability of RC structures. Corrosion of reinforcement is mainly accelerated by penetration of chloride ion in to concrete. Miyazato and Hiraishi (2005) studied the chloride ion penetration depth in reinforced concrete and reinforced ECC. For this purpose, reinforced concrete and ECC beams were preloaded to the same level in flexure followed by exposure to the accelerated corrosion using chloride solution. It was observed that the chloride penetration depth in reinforced ECC beams was significantly lower than reinforced concrete beams. In another study, Sahmaran et al. (2007) investigated the effective chloride diffusion coefficient of reinforced ECC and mortars under flexural deformation. In that study, specimens were immersed in 3\% NaCl solution in accordance with AASHTO T259 (2002). 
Reinforced ECC beams exhibited multiple micro-cracking behavior with crack width of less than $50 \mu m$ under high levels of flexural deformation resulted in substantially lower effective diffusion coefficient compared to reinforced mortar samples reaching crack width of $150 \mu \mathrm{m}$ under same level of loading. It was found by Sahmaran et al. (2007) that effective diffusion coefficient is more related to the crack width rather than number of cracks, attributed to the very fine crack width. In addition, chloride diffusion of ECC samples under 100,000 cycles of flexural loading was investigated by Oh and Shin (2006). It was observed that in spite of increasing number of cracks under flexural loading cycles, chloride diffusion coefficient of ECC specimens did not enhance considerably, which can be explained by multiple micro-cracking behavior and low crack width in ECC samples (Li, V.C., 2008).

\section{2-1-2-6-3 Absorption}

In practice, most of the time, reinforced concrete structures are not in fully saturated condition. In this case, transport properties of the concrete may not be governed by permeability or diffusion. Under dry or partially saturated conditions, the movement of water into concrete is controlled by capillary suction forces existing in the evacuated capillary cavities within the matrix (Martys and Ferraris, 1997). Sahmaran and $\mathrm{Li}$ (2009b) investigated the water absorption and sorptivity properties of virgin (uncracked) and mechanically preloaded (cracked) ECC samples in accordance with ASTM C642 (2006) and ASTM C1585 (2011). Water absorption test included immersion of pre-dried cracked and uncracked ECC samples in water until reaching the fully saturation condition, followed by measuring the mass of absorbed water per unit mass of pre-dried ECC samples. Also, sorptivity test was performed by allowing the pre-dried specimens to absorb water through capillary suction in one direction and measuring the enhancement in mass of the samples, which is quantified by sorptivity index. It was observed that increase in the number of micro- 
cracks in cracked ECC samples, resulted in fairly significant increase in the sorptivity index showing more vulnerability of the preloaded ECC specimens to water absorption compared to the virgin ECC samples. However, srptivity of the ECC samples loaded up to $1.5 \%$ tensile strain on the tension side was particularly high compared to the normal concrete, which can be attributed to the use of high volumes of fly ash, low water to cementitious materials, and elimination of coarse aggregates. It was also found by Sahmaran and Li (2009b) that use a water repellent admixture in ECC mix resulted in the substantial reduction in sorptivity index of the both virgin and pre-cracked ECC samples (Figure 2-10a), pointing out that presence and number of micro-cracks has a negligible effect on sorptivity index of ECC samples. Moreover, ECC samples containing water repellent admixture showed lower volume of permeable pores. It was also observed that increasing number of micro-cracks did not have a significant effect on the percentage of permeable pores in ECC samples.

It is worth-mentioning that self-healing ability of ECC, along with multiple micro-cracking behavior and tight crack width contributes to the low permeability, diffusion, and water absorption of the cracked ECC samples compared to normal concrete. It was observed by (Lepech and Li, 2005b; Sahmaran et al., 2007) that pre-cracked ECC specimens exposed to water or chloride solution showed closing of the micro-cracks due to self-healing resulted in lower rate of water or ion permeability, diffusion, and absorption. This can be explained by low water to cementitious materials ratio and use of high volumes of fly ash in ECC.

\section{2-1-2-7 Corrosion resistance}

Another deterioration mode that largely affects the load bearing capacity and service life of the reinforced concrete structures is corrosion of the reinforcement. Rebar corrosion is defined by 
degradation of the rebar through an electrochemical reaction with its environment. In general, in order to rebar corrosion to happen, four elements should be present: anode, cathode, electrical conductor, ion conductor (electrolyte). Anode, which is usually a metal, experiences the oxidation (anodic reaction) by releasing electrons and positive ions. Electrical conductor allows the released electrons to travel to cathode element, where they are consumed through reduction reaction. Electrolyte, which is usually an aqueous solution in contact with both anode and cathode, provides the opportunity for movement of ions between anode and cathode. In case of reinforced concrete member, in absence of dissimilar metals, rebar plays the role of both and cathode, with two points with different energy levels on the rebar. Also, concrete works as the electrolyte, and the electron are transferred trough wire ties, or the rebar itself (electrical conductor). In presence of moisture and oxygen, initial corrosion of the rebar results in formation of a passive film (protective layer) on top of the rebar surface. Passive layer is well protected by high alkalinity of the concrete $(\mathrm{pH}>$ 12.50) in presence of $\mathrm{Ca}(\mathrm{OH})_{2}$. The passive layer remains stable in a medium with a minimum $\mathrm{pH}$ of 11.5 and is largely affected by drop in the $\mathrm{pH}$ of the concrete. However, after the concrete cover is cracked due to excessive structural loading or restrained shrinkage or thermal deformation, aggressive agents such as chloride ions or carbon dioxide penetrates to the concrete cover, reaching the rebar surface, reducing $\mathrm{pH}$ of the concrete. By increasing the chloride concentration and reaching the thresholds, the passive film is destroyed on the rebar surface, accelerating the rebar corrosion. A number of methods are commonly used to solve or minimize the problem of reinforcement corrosion in $\mathrm{RC}$ members such increasing the concrete cover thickness, use of epoxy coated rebars, use of high-quality concrete along with proper compaction and curing regime. Increasing cover thickness can effectively reduce the rate of penetration of chloride ions, carbon dioxide, and moisture to the concrete until it is not cracked. However, cracking of concrete cover 
is inevitable in most cases, and higher thickness of concrete cover results in larger crack width and providing a pathway for ingress of aggressive agents reaching the rebar surface and accelerating corrosion. Furthermore, in case of epoxy coated bars, manufacturing defects as well as damage or scratch of the epoxy coating on the rebar surface during handling or placement reduced the efficiency and caused doubt reliability of the epoxy coating bars (Federal Highway Administration (FHWA), 1992; Sagues et al., 1994; Manning, 1996; Mavani, 2012). By incorporating ECC as a ductile HPFRCC with superior mechanical and durability properties including strain hardening, high tensile capacity, multiple cracking behavior, problems regarding penetration of aggressive agents, and corrosion of the rebars can be resolved or minimized.

Miyazato and Hiraishi, 2005 investigated the rate of corrosion in reinforced ECC (R/ECC) and reinforced concrete (R/C) beams. For this purpose, R/ECC and R/C beams were loaded followed by exposure to the chloride solution as the corrosion accelerator agent. Then, macro-cell and micro-cell corrosion rates were measured as an indicative of the corrosion rate in R/ECC and R/C beams. It was observed that summation of macro-cell and micro-cell corrosion rates was less than $0.004 \mathrm{~mm} /$ year in R/ECC beams, compared to the value of $0.0080 \mathrm{~mm} / \mathrm{year}$ in $\mathrm{R} / \mathrm{C}$ beams. In another study by Sahmaran et al. (2008), cracking behavior and residual flexural strength of R/ECC samples after accelerated corrosion was compared to reinforced mortars (R/mortar) specimens with the same compressive strength as ECC. In this respect, samples were exposed to chloride solution along with power supply to accelerate the corrosion process and corrosion induced cracks were monitored versus time. It was observed that reinforced mortar samples experienced crack width up to $2 \mathrm{~mm}$ along with considerable reduction in stiffness and flexural capacity. R/mortar specimens showed more than $65 \%$ reduction in flexural capacity after 25 hours of exposure to chloride solution. However, it was found by Sahmaran et al. (2008) that multiple 
micro-cracking behavior of ECC resulted in a large number of cracks along with crack width up to $0.1 \mathrm{~mm}$ at higher levels of corrosion. Moreover, R/ECC specimens recovered almost $100 \%$ of the initial flexural capacity after 50 hours of accelerated corrosion. R/ECC samples showed 55\% reduction in flexural capacity after 300 hours of accelerated corrosion. Figure 2-5 illustrates the $\mathrm{R} / \mathrm{ECC}$ and $\mathrm{R} / \mathrm{mortar}$ prisms and cylinders after various times of exposure to chloride solution.

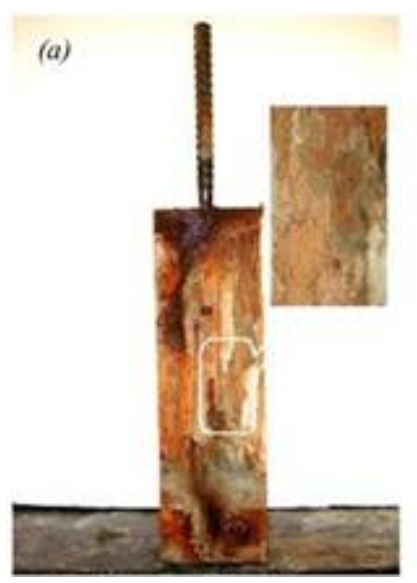

(a)

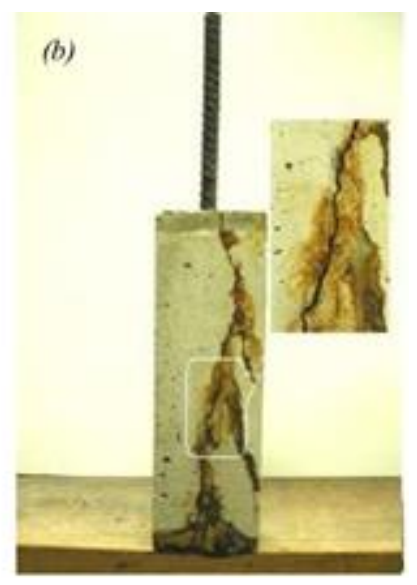

(b)

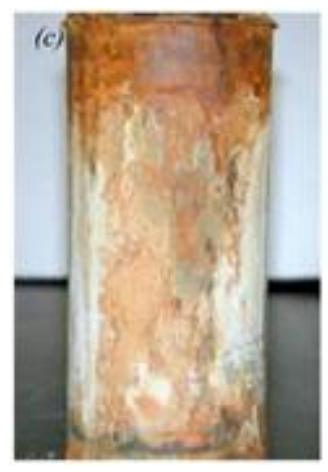

(c)

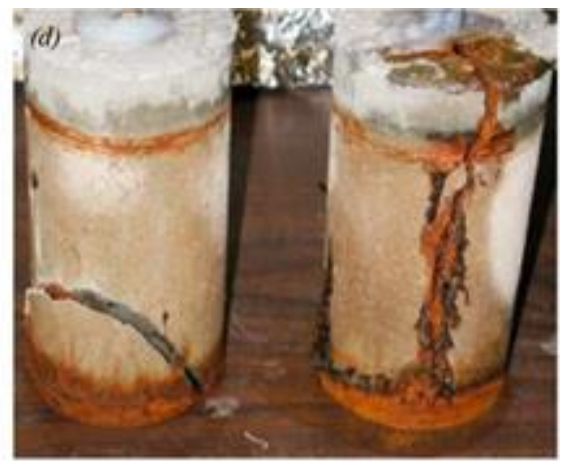

(d)

Figure 2-5: Reinforced ECC and mortar samples after exposure to accelerated corrosion: (a): R/ECC prisms after 300 hours of accelerated corrosion, (b): R/mortar prisms after 75 hours of accelerated corrosion, (c): R/ECC cylinder samples after 350 hours of accelerated corrosion, (d):

R/mortar cylinder samples after 95 hours of accelerated corrosion (Sahmaran et al., 2008; Mavani, 2012)

\section{2-1-3 Self-healing behavior of ECC}

ECC has a variety of unique properties including multiple micro-cracking behavior, high tensile ductility, strain hardening behavior along with self-healing ability. These superior properties are 
achieved by optimizing the interaction between the fibers and cementitious matrix through micromechanical tailoring of the ECC mix design. Presence of fibers in ECC helps through continuous development of closely spaced microcracks with tight crack width of around 60-100 $\mu \mathrm{m}$ even at the failure load, rather than developing a few number large cracks in conventional concrete. This multiple micro-cracking behavior leads to lower permeability, higher durability, as well as higher self-healing ability of ECC compared to conventional concrete. Self-healing ability of concrete materials, which is characterized by self-remediation of cracks in presence of water, results in gradual reduction of water flow rate passing through a crack and in extreme cases complete blockage of water flow by self-healing products. Self-healing of cracks in concrete materials occurs as a result of a series of chemical, physical, and mechanical processes including precipitation of calcium carbonate $\left(\mathrm{CaCO}_{3}\right.$ or calcite) and hydration of unreacted cementitious materials including both cement and supplementary cementitious materials such as fly ash and slag (Edvardsen 1999; Reinhardt and Jooss 2003; Sahmaran et al. 2008). Calcite formation and hydration of cementitious materials take place according to the following reactions:

$$
\begin{array}{lr}
\mathrm{C}_{2} \mathrm{~S} \text { or } \mathrm{C}_{3} \mathrm{~S}+\mathrm{H}_{2} \mathrm{O} \rightarrow \text { Primary } \mathrm{CSH}+\mathrm{Ca}(\mathrm{OH})_{2} & \text { Hydration reaction } \\
\mathrm{Ca}(\mathrm{OH})_{2}+\mathrm{SCMs}\left(\mathrm{SiO}_{2}\right)+\mathrm{H}_{2} \mathrm{O} \rightarrow \text { Secondary CSH } & \text { Pozzolanic reaction }
\end{array}
$$

$\mathrm{Ca}^{2+}+\mathrm{CO}_{3}^{2-} \rightarrow \mathrm{CaCO}_{3} \quad\left(\mathrm{pH}_{\text {water }}>8\right) \quad$ Calcite formation reaction

$\mathrm{Ca}^{2+}+\mathrm{HCO}_{3}^{-} \rightarrow \mathrm{CaCO}_{3}+\mathrm{H}^{+} \quad\left(7.5<\mathrm{pH}_{\text {water }}<8\right) \quad$ Calcite formation reaction

Tricalcium silicate $\left(\mathrm{C}_{3} \mathrm{~S}\right)$ and Dicalcium silicate $\left(\mathrm{C}_{2} \mathrm{~S}\right)$ are two of major compounds of cement, playing a significant role in strength gain of concrete. Upon exposure of the cracked section to water, unreacted cement particles, recently exposed due to crack formation, reacts with water to produce calcium silicate hydrate $(\mathrm{CSH})$ and calcium hydroxide $\left(\mathrm{Ca}(\mathrm{OH})_{2}\right)$ or release calcium ions 
$\left(\mathrm{Ca}^{2+}\right)$ and hydroxide ions $\left(\mathrm{OH}^{-}\right)$through the hydration reaction. Meanwhile, released calcium hydroxide reacts with silicone dioxide $\left(\mathrm{SiO}_{2}\right)$ component of the supplementary cementitious materials (fly ash, slag, etc) to further produce calcium silicate hydrate (CSH) through pozzolanic reaction. Simultaneously, calcium ions $\left(\mathrm{Ca}^{2+}\right)$ released from hydration reaction reacts with inwater available carbonates $\left(\mathrm{CO}_{3}^{2-}\right)$ or bio-carbonates $\left(\mathrm{HCO}_{3}^{-}\right)$leads to formation of water-insoluble calcite or calcium carbonate $\left(\mathrm{CaCO}_{3}\right)$. Achieving high levels of crack self-healing largely depends on presence of tight crack width as well as quantity of the available unreacted cementitious materials to participate in hydration and pozzolanic reactions to further produce calcium silicate hydrate (CHS) and calcite $\left(\mathrm{CaCO}_{3}\right)$. Self-healing products fill the crack and emerge as white scar on the concrete surface.

Self-healing of cracks results in blocking the water pathway through the crack and preventing transport of fluids as well as retaining the mechanical properties of the cracked concrete member including flexural/tensile/compressive strength, stiffness and modulus of elasticity (Fischer and Li, 2003; Herbert and Li, 2013; Li and Li, 2011; Yang et al., 2009). Self-healing ability has been observed in a variety of cementitious materials. However, failure in maintaining a certain level of crack width considerably reduce the self-healing effectiveness. ECC exhibits great self-healing potential through unique multiple micro-cracking behavior with tight crack width as well as high volumes of cementitious materials and low water to binder (W/B) ratio. A number of investigations on self-healing behavior of concrete materials are highlighted in this section.

Rashed et al. (2000) investigated the cracking and leakage behavior of prestressed RC elements under various combinations of axial tension and flexural loading. Each specimen represented the wall segment of water tank subjected to internal hydrostatic water pressure. For this purpose, a total number of eight full-scale prestressed RC specimens were fabricated and tested under pure 
tension and four-point loading conditions. The experimental parameters included various prestressed and non-prestressed reinforcement ratios along with different combinations of loading conditions. Followed by crack formation in specimens, leakage test was performed under sustained loading and experimental results were recorded and compared. It was found by Rashed et al. (2000) that partial prestressing significantly improved the cracking behavior of the specimens under pure flexure and pure tension. No leakage was observed in specimens under flexure due to presence of flexural compression zone. In specimens under pure tension, leakage initiated after formation of full-depth cracks. However, full-depth cracks with effective width less than $0.15 \mathrm{~mm}$ self-healed after less than two-days of water exposure. The study highlighted the importance of limiting crack width in liquid containing structures through partial prestressing so that self-healing of cracks can occur.

Ziari and Kianoush (2008) performed a study on cracking, leakage and self-healing behavior of RC panels exposed to monotonic direct tensile loading and hydro-static water pressure. For this purpose, one RC panel, which represent the wall segment of a tank, was subjected to direct tensile loading and cracking behavior of the specimen was monitored. In the next step, the major direct tension crack was exposed to pressurized water and leakage rate was recorded for 30 hours to evaluate the leakage and self-healing behavior of the RC panel. It was observed that self-healing of tension cracks due to hydration of unreacted cementitious materials resulted in reducing the leakage rate of $\mathrm{RC}$ panel over time. It was found that tension cracks as wide as $0.25 \mathrm{~mm}$ are capable of partial self-healing in exposure to pressurized water under sustained tensile load.

Yang et al. (2009) investigated the self-healing behavior of ECC under two different cyclic wetting and drying regimes. For this purpose, resonant frequency measurements, uniaxial tensile tests, and water permeability tests were performed to examine the rate and extent of self-healing in ECC 
specimens in terms of mechanical and transport properties recovery. Two cyclic wetting and drying regimes were used in that study including one cyclic (CR1) regime to simulate outdoor environments such as rainy and uncloudy days as well as another cyclic regime (CR2) to simulate outdoor environments alternating between rainy days and days with sunshine and high temperature. Specimens with dimension of $230 \mathrm{~mm}$ by $76 \mathrm{~mm}$ by $13 \mathrm{~mm}$ were used in this study. ECC specimens were preloaded up to various uniaxial tensile strain levels from 0.35 to $3 \%$ at the age of 6 months. In the next step, specimens were exposed to ten wetting and drying cycles. It was found that limiting the crack width below $0.15 \mathrm{~mm}$ in cementitious materials was of prime importance to achieve high levels of self-healing. It was observed by Yang et al. (2009) that ECC cracked specimens retained $76 \%$ to $100 \%$ of their original resonant frequency value and achieved a considerable rebound in stiffness. The study confirmed great self-healing potential of ECC in exposure to wetting and drying cycles, as illustrated in Figure 2-6.

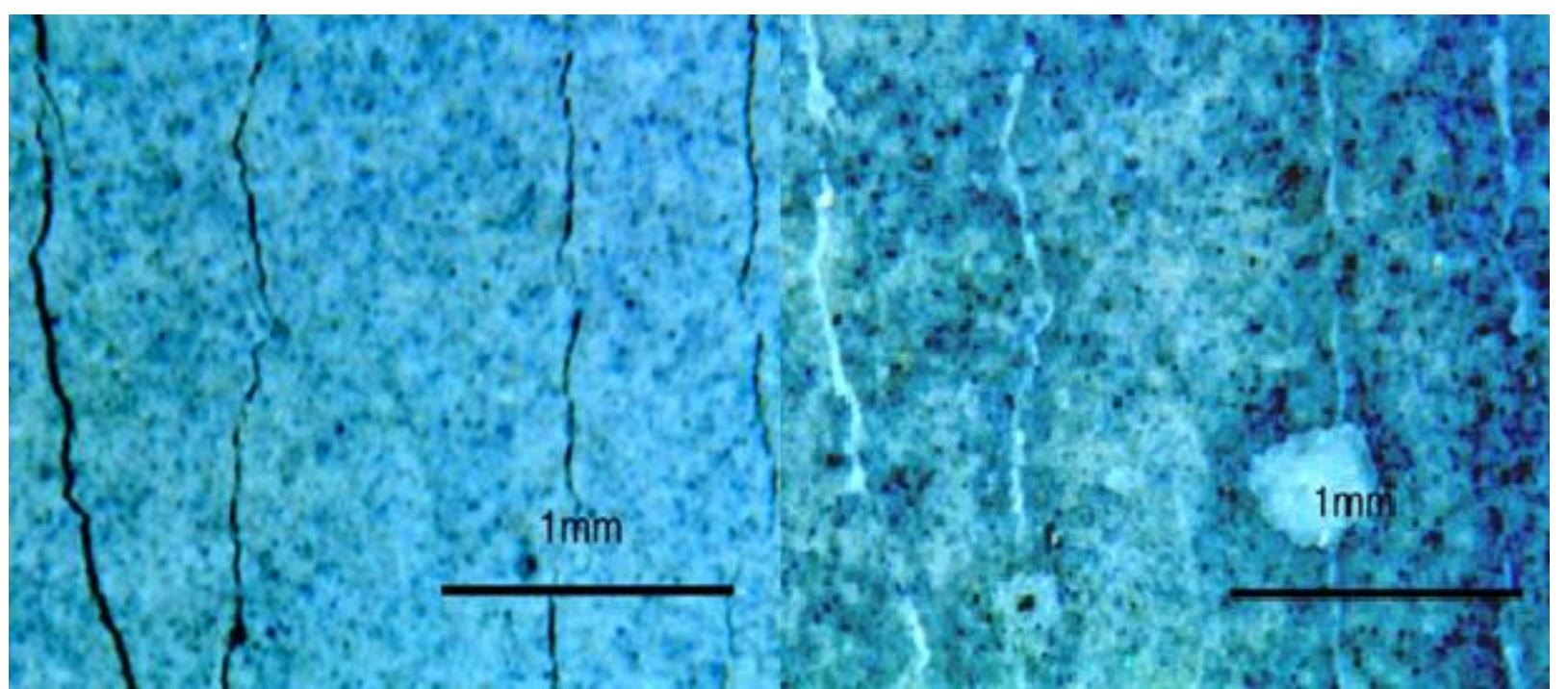

a) b)

Figure 2-6: Self-healing of micro-cracks in ECC specimens a) Before self-healing b) After selfhealing, (Yang et al. 2009)

Zhang and Zhang (2017) performed a study on the effect of various environmental exposures including hot water $\left(60^{\circ} \mathrm{C}\right)$ and calcium hydroxide solution $\mathrm{Ca}(\mathrm{OH})_{2}$ on self-healing behavior of 
ECC containing high volumes of fly ash. Samples of cement-fly ash paste (without silica sand and PVA fiber) with various fly ash to cement ratio of $1.2,2.2$, and 4 along with constant water to cementitious materials at 0.25 were prepared and tested. Compressive strength, non-evaporable water content, and fly ash reaction degree of the cement-fly ash paste was measured at the age of 28-day. Moreover, ECC samples with fly ash to cement ratio of 2.2 were prepared and tested. Water flow test, uniaxial tension test, and ESEM observations were performed to reflect the selfhealing behavior of ECC under various exposure environments. It was found that hot water $\left(60^{\circ}\right.$ C) and calcium hydroxide solution $\mathrm{Ca}(\mathrm{OH})_{2}$ improved the self-healing behavior of both cementfly ash paste and ECC specimens by increasing the cement hydration and fly ash pozzolanic reactions, while hot water $\left(60^{\circ} \mathrm{C}\right)$ was found to be more effective. Such a conclusion was confirmed by ESEM observation, as shown in Figure 2-7. ESEM images clearly showed higher amount of self-healing products filling the crack in ECC samples exposed to hot water $\left(60^{\circ} \mathrm{C}\right)$ and calcium hydroxide solution $\mathrm{Ca}(\mathrm{OH})_{2}$ compared to $20^{\circ} \mathrm{C}$ water. It was also concluded that self-healing mechanism resulted in recovery of the tensile stiffness of the ECC samples. However, self-healing ability of ECC was observed to decrease over time due to less available cementitious materials over time to participate in self-healing.

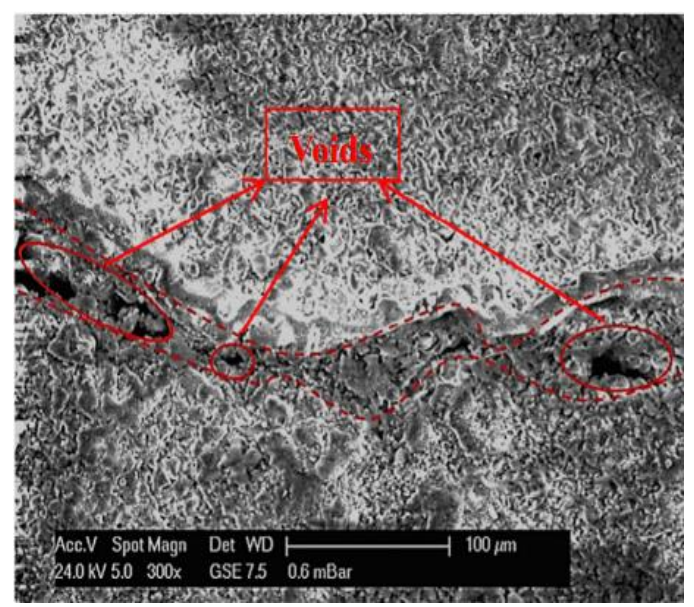

a) 


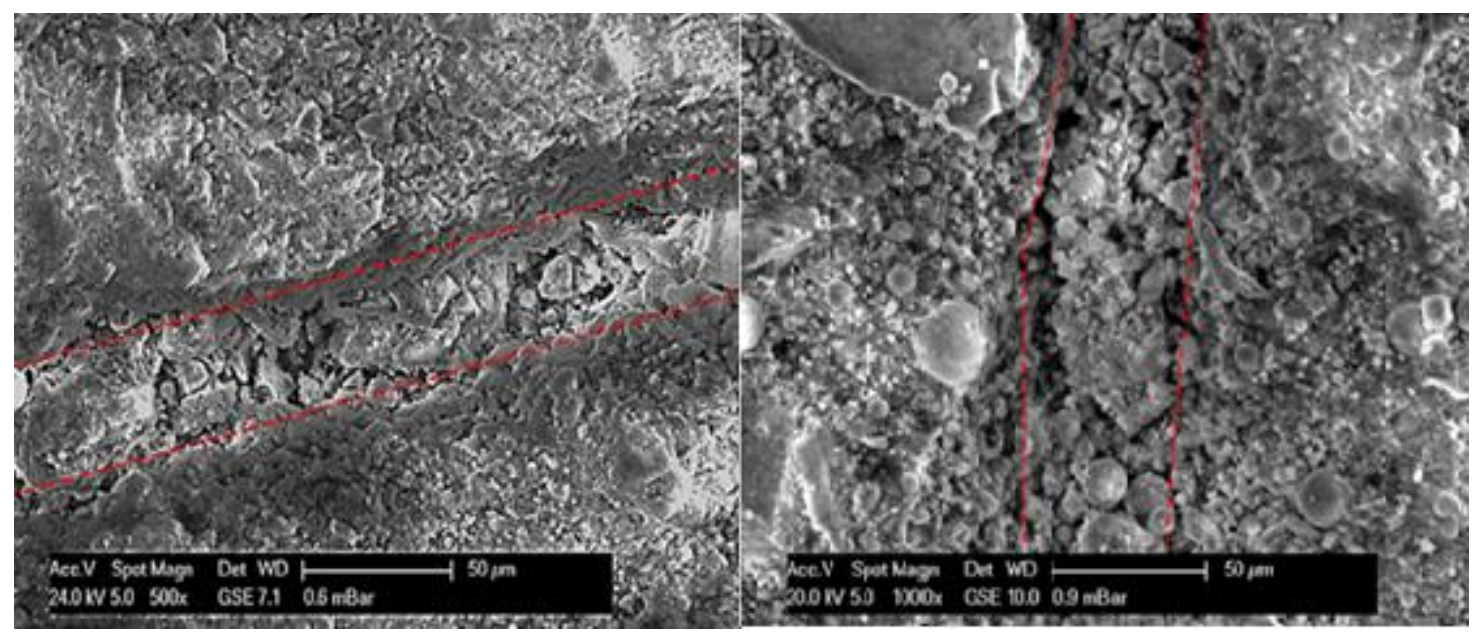

b)

c)

Figure 2-7: Self-healing products within the crack exposed to a) $20^{\circ} \mathrm{C}$ water b) $60^{\circ} \mathrm{C}$ water c) Saturated calcium hydroxide solution $\mathrm{Ca}(\mathrm{OH})_{2}$, (Zhang and Zhang, 2017)

Hooshmand et al. (2019) investigated the self-healing behavior of slag ECC and NC panels under direct tensile loading. RC panels represented wall segment of a tank exposed to internal water pressure. Two RC panels were fabricated and tested under pure tension to develop full-depth cracks at pre-determined locations. In the next step, leakage test was performed and leakage rate of the specimens was recorded over the time to study the self-healing behavior of the NC and ECC panels. It was observed that $\mathrm{NC}$ panel experienced cracks as wide as $0.4 \mathrm{~mm}$ and $0.55 \mathrm{~mm}$ which was followed by high rate of leakage. However, due to multiple micro-cracking behavior of ECC, micro-cracks with maximum width of $0.15 \mathrm{~mm}$ and $0.2 \mathrm{~mm}$ formed in ECC specimen which resulted in a much lower leakage rate compared to $\mathrm{NC}$ panel. It was found that ECC panel exhibited the capability of self-healing the cracks and blocking the water flow through the cracks, while NC panel showed just partial self-healing of cracks. The study confirmed superior leakage and selfhealing behavior of ECC, due to multiple micro-cracking behavior and large quantity of available cementitious materials, compared to NC counterparts. 


\section{2-2 Application of ECC in construction and repair of real-world structures}

Applicability of ECC in construction and repair of real-world full-scale structures are briefly emphasized in this part. Figure 2-8 shows a gravity retaining wall in Gifu, Japan with approximate dimensions of $18 \mathrm{~m}$ width and $5 \mathrm{~m}$ height, which was built in 1970s and experienced deterioration due to alkali silica reaction (ASR) (Rokugo et al., 2005).

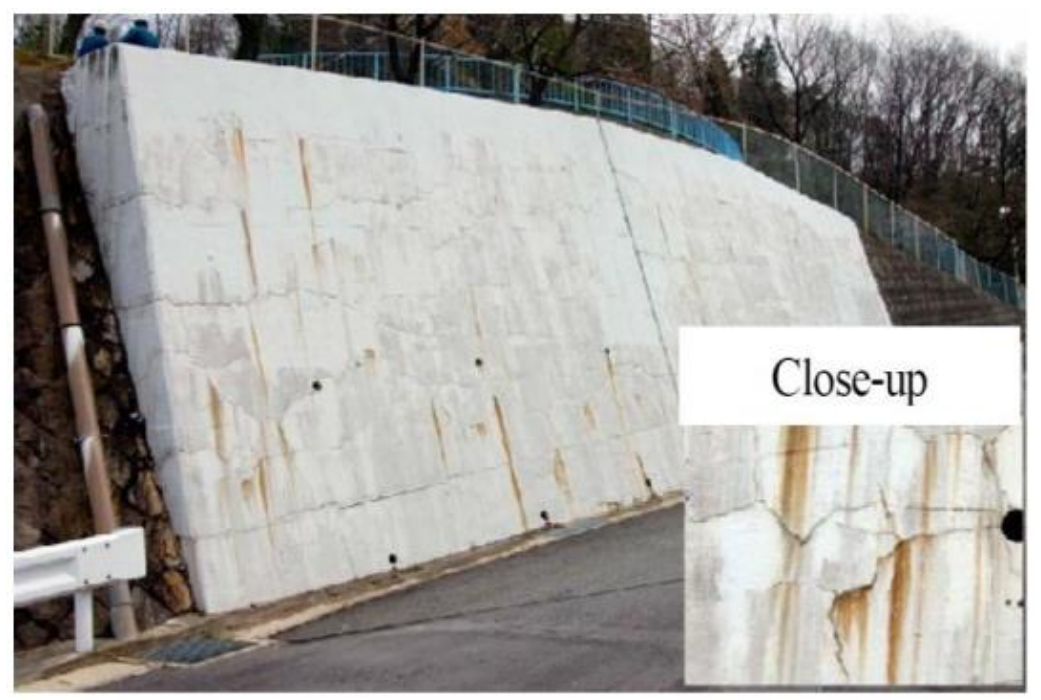

Figure 2-8: Deterioration due to Alkali-silica reaction (ASR) in a gravity retaining wall in Gifu, Japan (Rokugo et al., 2005; Yeganeh, 2013)

Due to extensive cracking inside the wall and in order to minimize the probability of crack reflection in to the repair layer, a 50 to $70 \mathrm{~mm}$ thickness layer of ECC was chosen to be applied. For this purpose, the wall was divided into 10 blocks including 8 blocks repaired with ECC (4 blocks using ECC containing 1.5\% of hybrid PVA and PE fibers, 4 blocks using ECC with 2.1\% PVA fiber), 1 block using normal concrete, and 1 blocked remained without any repair. As the repair program was performed in 2003 , the cracking condition of the wall was monitored for two years (24 months). The ECC repaired blocks experienced no cracking until 7 months, while NC repaired blocks showed visible cracks after one month. Moreover, crack width in the ECC repaired blocks remained less than $50 \mu \mathrm{m}$ and $120 \mu \mathrm{m}$ at 10 and 24 months of repair program, respectively. 
However, blocks repaired with NC experienced crack widths of $200 \mu \mathrm{m}$ and $300 \mu \mathrm{m}$ at 10 and 24 months, respectively. Figure 2-9 illustrates the cracking behavior and condition of the wall after the repair using ECC and $\mathrm{NC}$ after 12 and 24 months. Blocks 1-8 were repaired using ECC, block 9 using NC, and block 10 without repair.

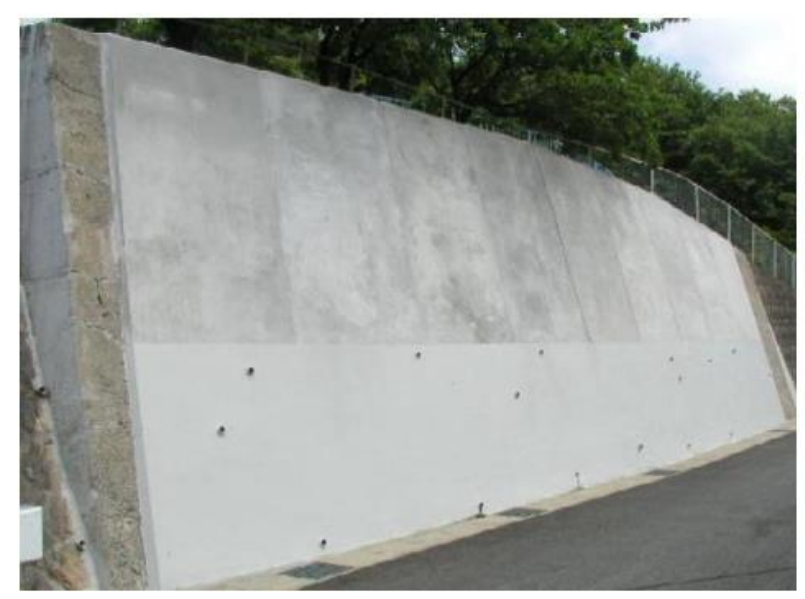

(a)

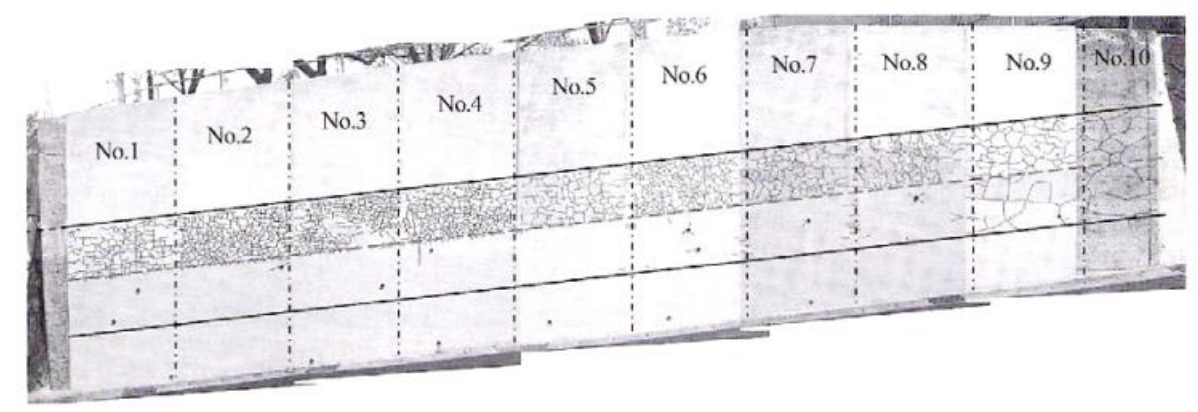

(b)

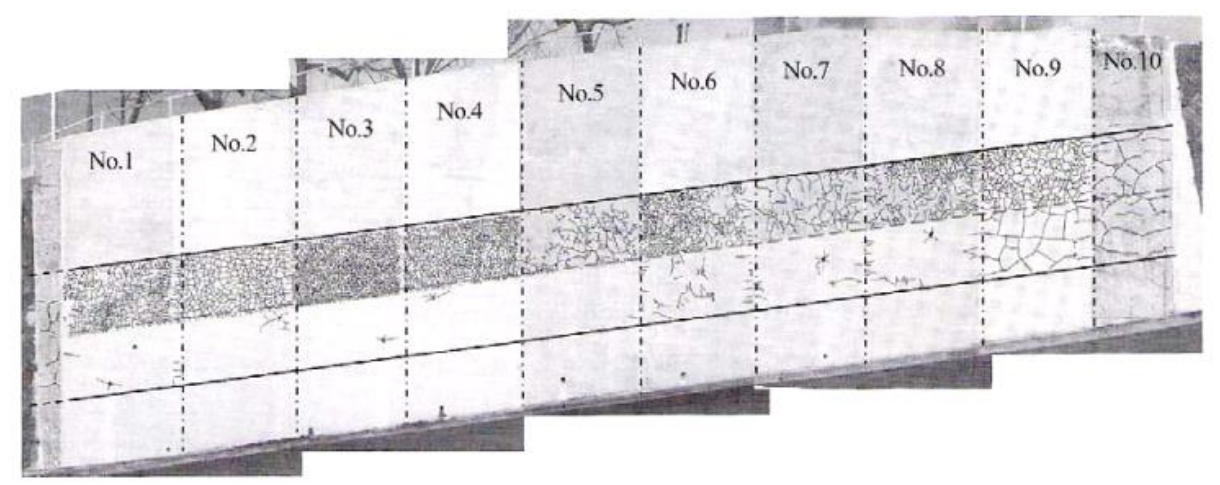

(c)

Figure 2-9: Repair of a gravity retaining wall using ECC (a) Condition of the wall after the repair program (b) Cracking behavior of the wall after the repair at 12 months (c) Cracking behavior of the wall after the repair at 24 months (Rokugo et al., 2005) 
In another project, ECC was used as patch repair for the bridge deck of the Curtis Road over M14 in Southern Michigan in 2002. A complete summary of this repair project, which was performed in cooperation with the Michigan Department of Transportation (MDOT), has been presented by $\mathrm{Li}$ and Lepech (2004). In this project, two types of materials were used, including ECC and a concrete patching material commonly used by MDOT, and performances were compared under the same loading and environmental conditions (Figure 2-10a). A large number of heavily loaded 11 axle trucks travel on this road. After observing the crack width on the repaired bridge for more than 6 Michigan Winter freeze thaw cycles. The performances of ECC and concrete repair layers is illustrated in Figure 2-10b. It was found that ECC repaired layer showed micro-cracking behavior with crack width limited to $30 \mu m$ under combined loading and environmental condition, while concrete repair layer experienced cracks as wide as $3.8 \mathrm{~mm}$ at 780 days after the repair.

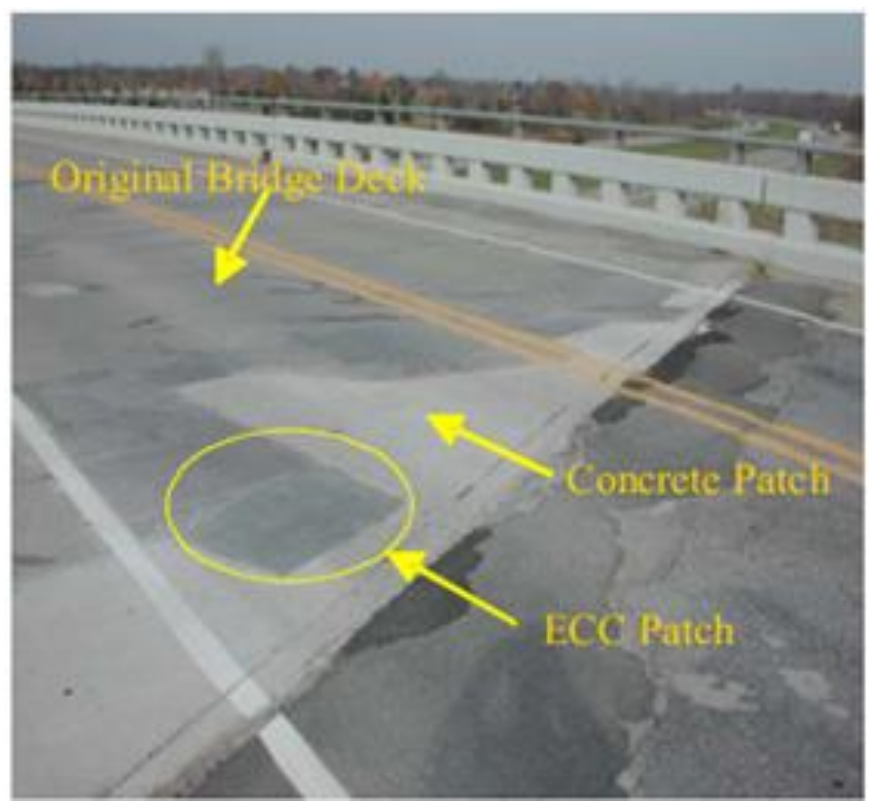

(a) 


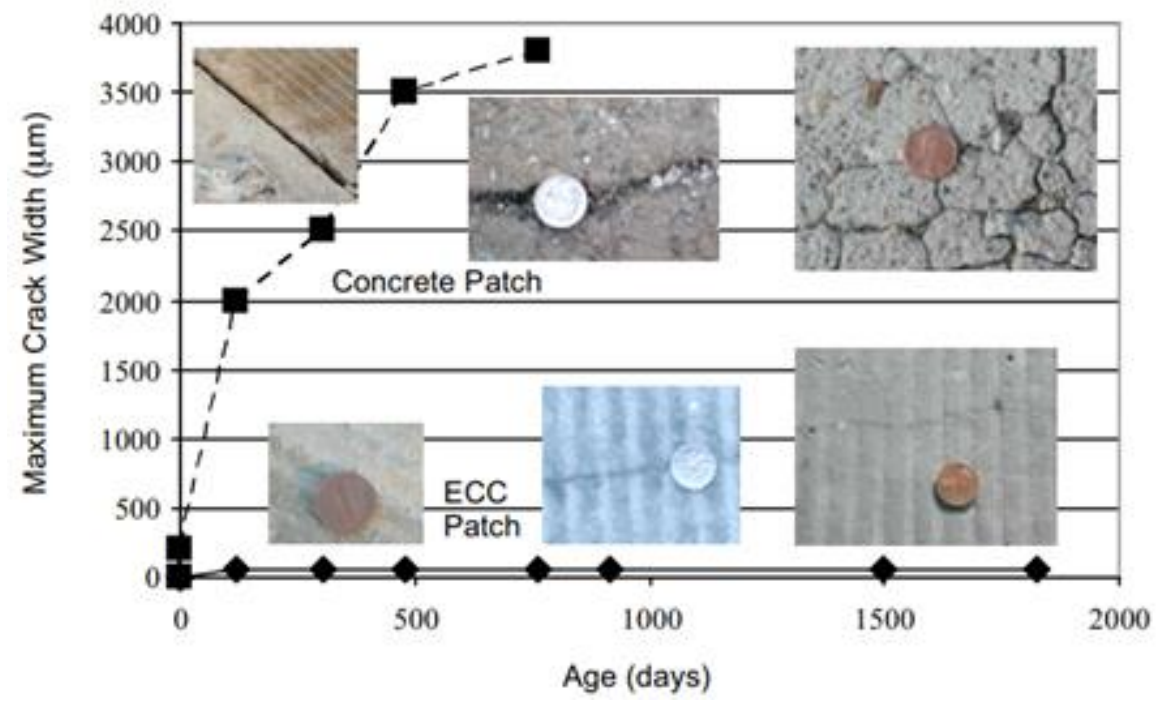

(b)

Figure 2-10: ECC repair application (a) Patch repair of bridge deck of the Curtis Road over M-14 in Southern Michigan (b) Crack width development versus time in ECC and concrete repair layers (Li and Lepech, 2004; Yeganeh, 2013)

The next real-life application of ECC was for Mihara Bridge in Hokkaido, Japan with a bridge length of $972 \mathrm{~m}$ and central span of 340 m, as shown in Figure 2-11 (Mitamura et al., 2005). In this project, which was performed in 2004, half of the asphalt overlay was replaced by ECC layer with $40 \mathrm{~mm}$ thickness. The project required $800 \mathrm{~m}^{3}$ of ECC and the bridge was opened to traffic in 2005. Using ECC/steel deck composite section along with micro-cracking behavior and high tensile ductility of ECC resulted in improving the structural performance of the deck, reducing the weight and materials by $40 \%$, as well as elimination of the expansion joints.

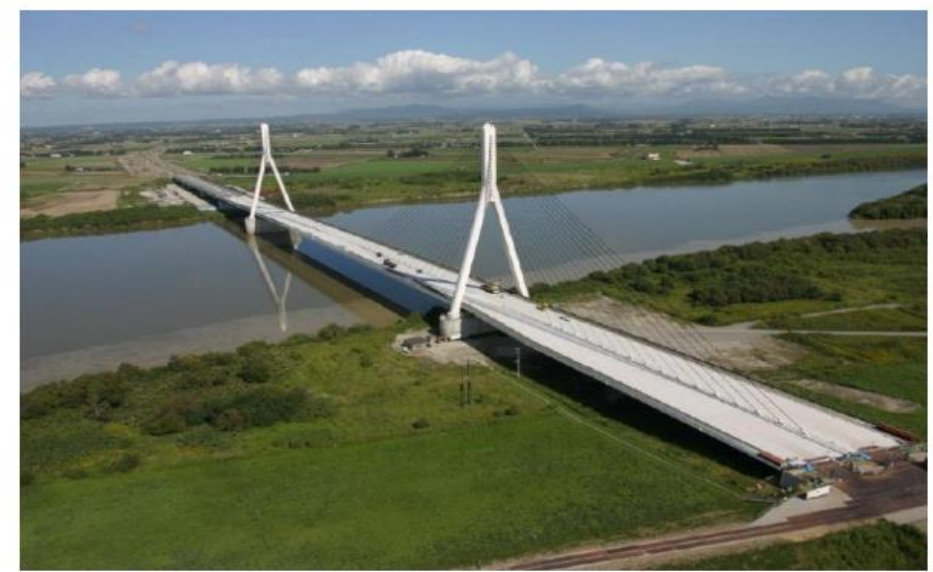

Figure 2-11: Mihara Bridge in Hokkaido, Japan (Mitamura et al., 2005) 
Another repair project performed in full-scale using ECC was the repair of Mitaka Dam in Hiroshima-Prefecture, Japan in 2003 (Kojima et al., 2004). Mitaka dam, which is an over 60-year old structure, was suffering from different modes of deterioration including cracking and concrete spalling resulted in leakage of water. In this repair project a $20 \mathrm{~mm}$ layer of ECC was used to be sprayed directly over a $600 \mathrm{~m}^{2}$ of the dam surface, as illustrated in Figure 2-12.

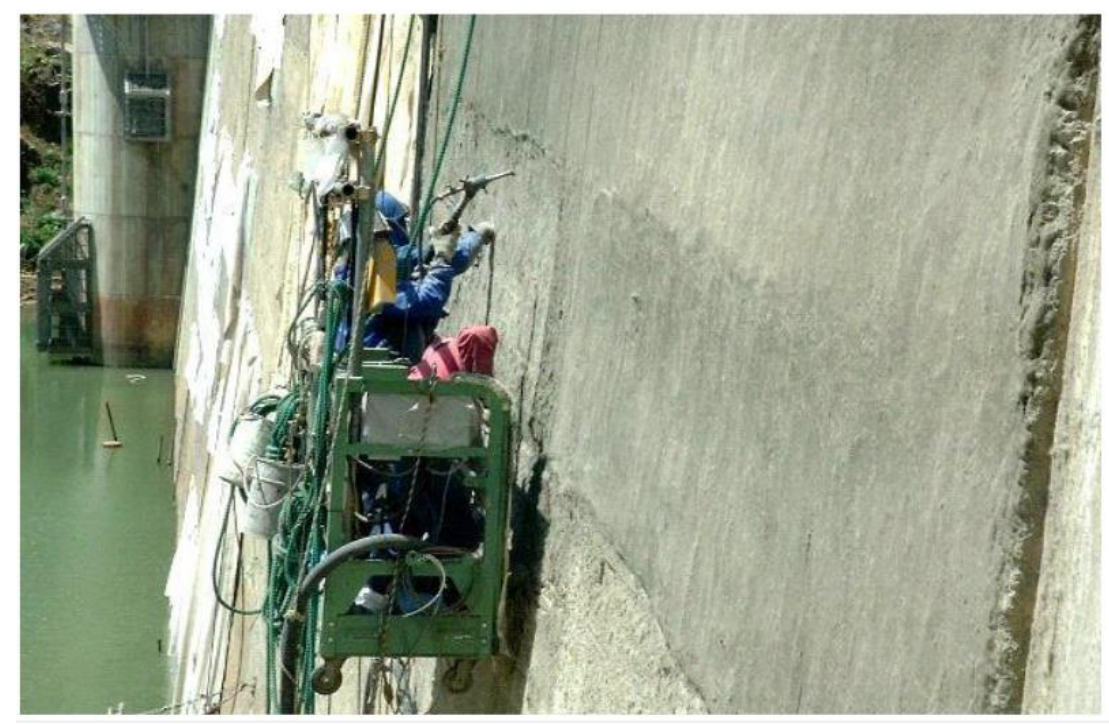

Figure 2-12: Water-proofing of Mitaka Dam in Hiroshima-Prefecture, Japan in 2003 by spraying ECC repair layer (Kojima et al., 2004)

Another field application of ECC is in the construction of the bridge link slab. Figure 2-13 shows the casting the link slab of the Grove Street Bridge in Southeast Michigan using ECC in 2005 by collaboration of MDOT. The purpose of this project was to use ECC link slab as a replacement for typical bridge deck expansion joints in simply supported multi-span bridges. A volume of around $32 \mathrm{~m}^{3}$ of ECC was used in this project to be cast in place using standard ready-mix concrete trucks. By benefiting from superior mechanical performance of ECC including high ductility and tensile strain capacity of above $2 \%$, ECC link slabs can successfully tolerate thermal deformation of adjacent spans in a multi-span bridge. Therefore, it can be used as an appropriate choice for traditional bridge expansion joints. 


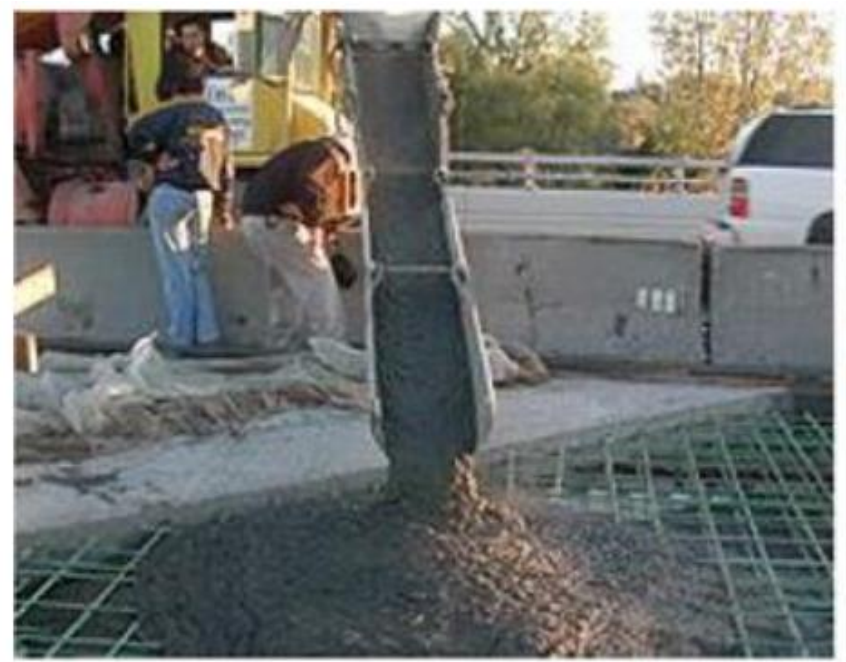

(a)

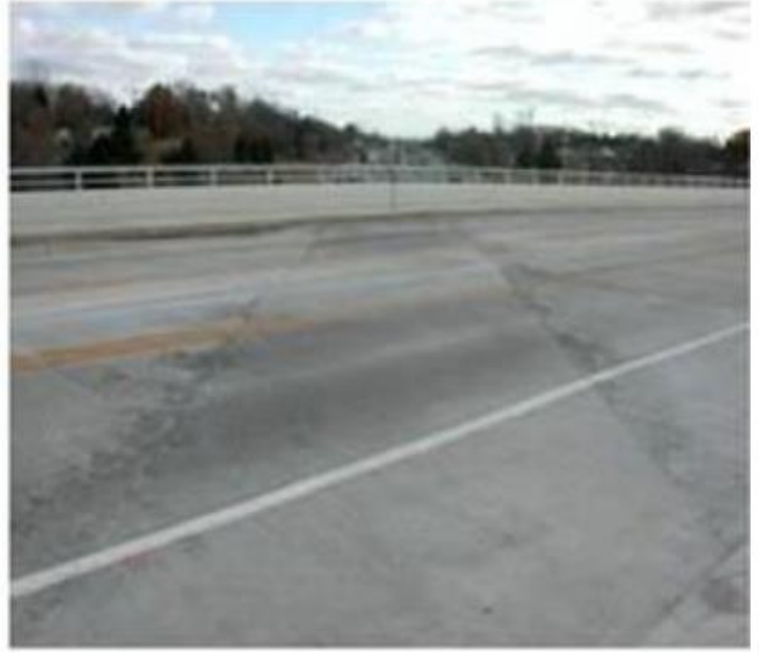

(b)

Figure 2-13: ECC link slab of Grove Street Bridge, Michigan, USA a) ECC casting b) Finished surface (Qian et al., 2009)

As another application of ECC in real-world structures, it was used as the coupling beams in construction of high-rise concrete structures. (Maruta et al., 2005). A coupling beam is an element connecting two core walls in high-rise buildings. Use of a ductile material with high levels of energy absorption such as ECC can be very advantageous as a coupling beam in high-rise buildings, especially in zones with high seismic risk. Precast ECC coupling beams have been used in building core for seismic resistance in 41-story Nabeaure and Glorio Roppongiin Yokohoma towers in Yokohoma, Japan (four coupling beams per floor), as shown schematically in Figure 214 (Yeganeh, 2013). 


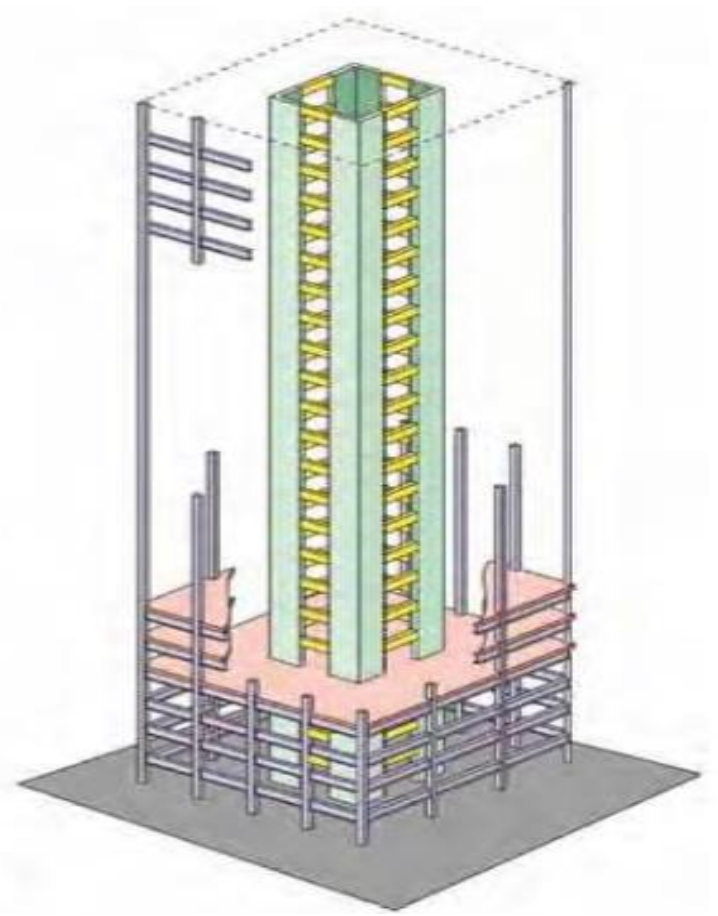

Figure 2-14: Schematic of using ECC coupling beams (in yellow) in high-rise buildings (Maruta et al., 2005; Yeganeh, 2013)

\section{2-3 Various techniques of repair and strengthening of $\mathrm{RC}$ members}

Civil infrastructures have been experiencing various modes of deterioration during the last decades due to low tensile strength and high permeability of NC. In most cases, partial or complete demolition and reconstruction of the deteriorated structure is neither economical, environmental friendly, nor practical. Therefore, the need for repair and rehabilitation seems to be inevitable. A number of repair and retrofitting techniques have been used over the years under various loading conditions with different levels of efficiency including section enlargement, shotcrete overlay, cement grouting, ferrocement layer, chemically or mechanically bonded steel plates, epoxy injection, concrete jacketing using high-strength concrete or self-compacting concrete, fiber reinforced polymer (FRP) laminates, engineered cementitious composite (ECC) jacketing, fiber reinforced cementitious mortars (FRCM) or textile reinforced mortars (TRM). In this section research conducted on repair and strengthening of $\mathrm{RC}$ members is reviewed with a concentration 
on repair applications of epoxy injection, FRP laminates, ECC and combined application of ECC with FRP grids.

Issa and Debs (2005) performed study on repair of cracks in concrete cube sample using gravity filled epoxy. Fifteen cube concrete samples were used including 6 cracked samples without repair, 6 cube samples repaired with epoxy, and 3 virgin samples without any crack. Epoxy repair was applied to the cubes using a low viscosity epoxy and gravity filling method. Applying epoxy by gravity filling was possible due to large artificial cracks, as wide as $2 \mathrm{~mm}$, introduced to the cube samples. Samples were crushed under compression and compressive strength was recorded for each sample. It was concluded by Issa and Debs (2005) that introduction of wide cracks to the cube samples significantly reduced the compressive strength by up to $40.93 \%$, while repair of cracks samples with gravity filled epoxy resulted in recovering the structural strength by decreasing the reduction down to $8.23 \%$. The study confirmed the applicability of the epoxy in restoring the structural capacity of the damaged concrete members.

In another study performed by Thanoon et al. (2005), cracked one-way slabs were repaired using different repair techniques such as cement grout, epoxy injection, ferrocement layer, CFRP strip, and section enlargement and efficiency of the repair materials were compared. For this purpose, 6 full-scale RC slabs were fabricated and tested under two line-load located at middle third of the specimen. All Specimens were loaded until development of cracks or up to $2 / 3$ of the capacity, except for the control sample, which was loaded until failure. Next, specimens were unloaded and followed by applying the repair materials. The repaired slabs were loaded up to failure and performance of each repair material was evaluated in terms of load- deflection response, strain development in concrete and steel, load bearing capacity, and failure mode. It was found that all repair materials were capable of restoring or improving the structural strength of the damaged $\mathrm{RC}$ 
slab. Specimens repaired with cement grout, epoxy injection, and ferrocement layer recovered capacity of the cracked RC slab and showed similar behavior as the control sample in terms of strength and ductility. However, RC slabs repaired with CFRP strip and section enlargement improved the structural strength by $77 \%$ and $130 \%$, respectively, while showed lower levels of ductility compared to the control sample. It is interesting to note that premature failure due to peeling of CFRP strip was observed followed by a sudden failure, as shown in Figure 2-15. This indicated the premature failure of the CFRP repaired specimen which was governed by peeling of the CFRP strip rather than achieving the full capacity and failure by rupture of the CFRP strip.

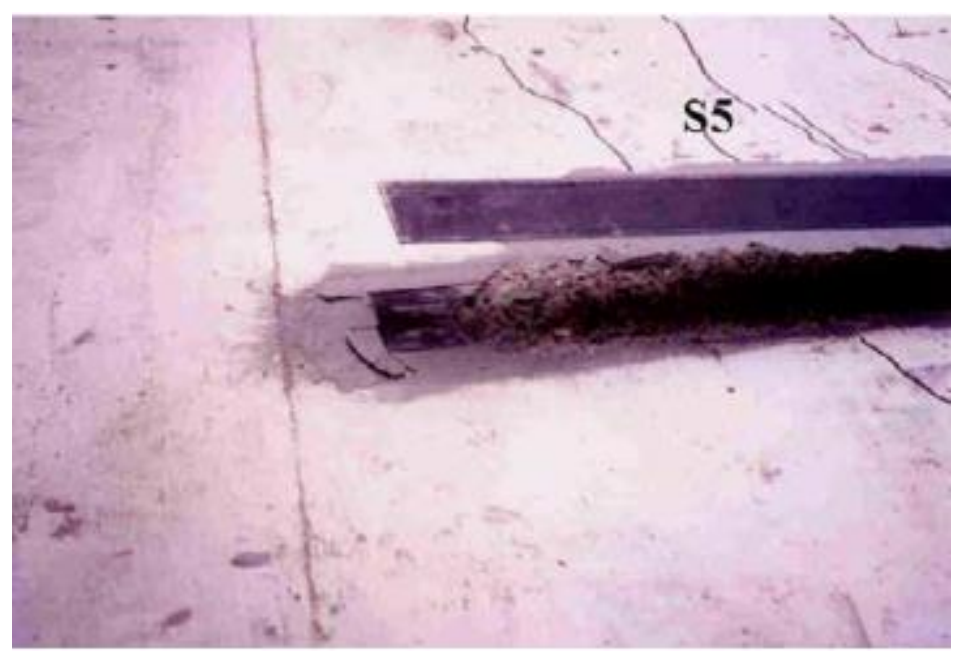

Figure 2-15: Premature failure due to CFRP strip peeling (Thanoon et al., 2005)

Higgins et al. (2007) investigated the effectiveness of epoxy injection in repairing of diagonally cracked full-scale conventionally reinforced concrete (CRC) deck girders under combined transverse loading and axial tension. For this purpose, 7 CRC deck girders were fabricated and tested to create diagonal cracks and subsequently repaired with epoxy injection under various levels of axial tension and service loading. The repaired samples were tested and experimental results were recorded and compared to control samples. It was found by Higgins et al. (2007) that use of epoxy injection repair technique resulted in recovering the structural capacity, improving the stiffness of the CRC deck girders, along with enhancing the load at which diagonal cracks 
started to form. Addition of cyclic live loading during the injection and curing of the epoxy introduced some bubbles in the epoxy matrix, while these bubbles did not affect the performance of epoxy injected specimens. The study confirmed the applicability of epoxy in retaining the structural strength of the diagonally cracked CRC deck girders under cyclic transverse loading and axial tension.

Ahmad et al. (2013) performed investigation on the application of epoxy injection in improving the structural performance of the cracked RC beams in flexure under four-point loading conditions. A total number of 6 full-scale RC beams were fabricated and tested in flexure until development of crack width as wide as $1 \mathrm{~mm}$. Specimens had a cross section of $152.4 \mathrm{~mm}$ width by $304.8 \mathrm{~mm}$ height along with the length of $3351 \mathrm{~mm}$. In the next step, beams were repaired using epoxy injection and were left for water curing for 3 days. Repaired beams were tested in flexure and loaded up to failure. A significant improvement in load bearing capacity along with a reduction in deflection was observed for epoxy repaired specimens compared to beams before repair. The study confirmed effectiveness of the epoxy injection method in reparing of the damaged RC members in flexure.

Griffin et al. (2017) investigated the effectiveness of epoxy in recovering the structural strength of the cracked unreinforced concrete beams under flexural loading. Three types of epoxy with different properties were used in this experimental program. A total number of 6 unreinforced concrete beams measuring $505 \mathrm{~mm}$ in length and $105 \mathrm{~mm}$ in height were tested. Width of the beams varied from $100 \mathrm{~mm}$ at the bottom to $105 \mathrm{~mm}$ on top. Specimens were tested under fourpoint loading until fracture or formation of cracks in tensile region. In the next step, beams were repaired with epoxy using gravity filling and low-pressure injection methods. Type 1 epoxy had high viscosity of 250-500 mPas, flexural tensile strength of $45 \mathrm{MPa}$, and compressive strength of 
$70 \mathrm{MPa}$. Type 2 epoxy had compressive strength of $70 \mathrm{MPa}$ and flexural tensile strength of 45 MPa, while the viscosity of type 2 epoxy was not reported. Type 3 epoxy had low viscosity of 145 $\mathrm{mPas}$, and highest flexural tensile strength of $60 \mathrm{MPa}$ and compressive strength of $75 \mathrm{MPa}$. Type 3 epoxy was applied using gravity filling method, while type 1 and type 2 epoxies were applied using low-pressure injection method. Repaired samples were tested under flexural loading until failure. It was found by Griffin et al. (2017) that failure of the beams repaired with type 1 epoxy occurred at the initial crack section, while failure in beams repaired with type 2 and type 3 epoxies occurred at a different location from the original fracture line, as shown in Figure 2-16.

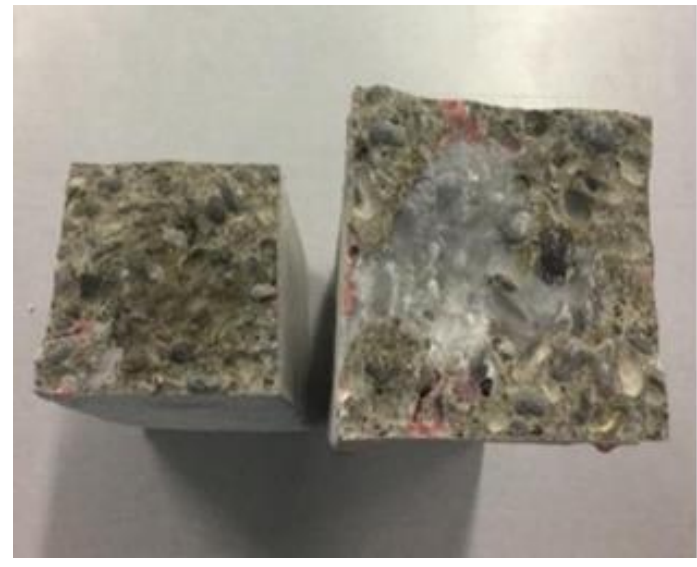

(a)

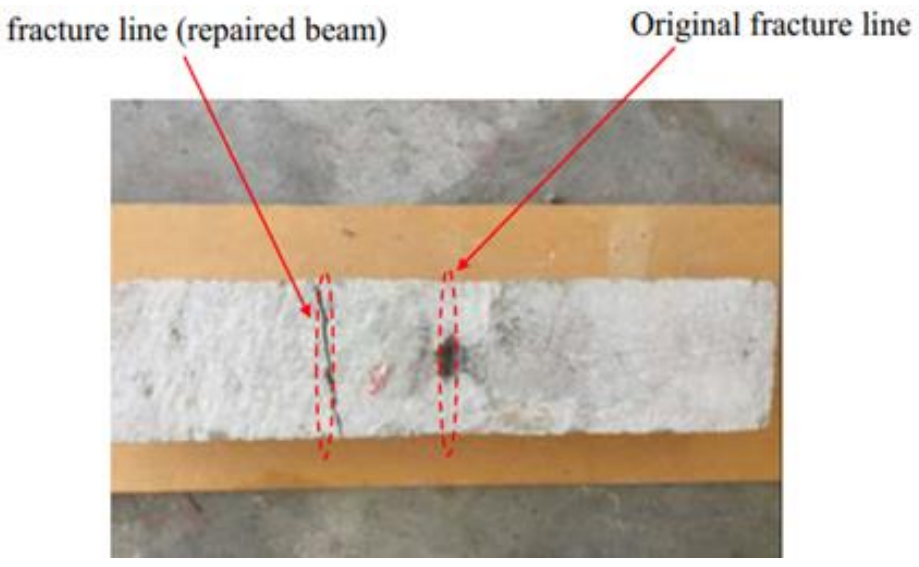

(b)

Figure 2-16: Failure of the beams repaired with epoxy a) Failure of the type 1 epoxy at original cracked section b) Failure of type 2 and type 3 epoxies at different location from original fracture line (Griffin et al., 2017)

It was concluded by Griffin et al. (2017) that type 2 and type 3 epoxies showed better performance by restoring the structural strength of the beams, while beams repaired type 1 epoxy achieved $71 \%$ of the failure load of the beams before repair. The study confirmed the effectiveness of epoxy in recovering the structural strength of damaged concrete members and also highlighted the importance of epoxy viscosity in a repair program using epoxy.

Hag-Elsafi et al (2000) investigated the effectiveness of FRP laminates in strengthening of RC Tbeam bridge structure. The repair program included repairing of freeze-thaw cracking and 
improving shear and flexural strength of the bridge structure. 9 beams out of 26 beams of the bridge structure were selected and instrumented to be incorporated in the experimental program. Four trucks each having $196 \mathrm{kN}$ were used in the load tests. Load tests were performed before and after applying the FRP laminate system. Based on the experimental results, it was concluded by Hag-Elsafi et al. (2009) that loading range which was used in the experimental program was not sufficient to fully utilize the load bearing capacity of the FRP laminate system. However, feasibility of applying FRP laminate to a real-world bridge structure with minimum disruption to traffic would exhibit applicability of FRP laminate strengthening system in repair of real-world structures in densely populated metropolitan areas.

Karayannis and Sirkelis (2008) performed a study on the repair of RC beam-column joints by incorporating CFRP jacketing and epoxy injection under cyclic axial load applied to the column. A total number of 12 external beam-column joint connection sub-assemblages were repaired and tested under cyclic loading. Two repair methods were used, including epoxy injection and combination of epoxy injection and CFRP sheet external wrapping. CFRP was also used to wrap critical regions of the beam and column members of the specimen to provide confinement for these regions as well as providing anchorage for FRP sheets used to warp the joint body. Based on the experimental results, it was concluded by Karayannis and Sirkelis (2008) that use of epoxy injection method could recover the structural strength of the damaged joint under cyclic loading and also failure occurred outside of the repaired area injected with epoxy, as shown in Figure 217. Moreover, simultaneous application of epoxy injection and CFRP sheets jacketing significantly improved the structural capacity, ductility, and energy absorption of the beam-column joint connection under cyclic loading. Damages in the repaired specimen using epoxy injection and CFRP jacketing occurred away from the joint near the end of the repaired area wrapped by CFRP 
sheets, as illustrated in Figure 2-18. The study highlighted the effectiveness of the epoxy injection, and combination of epoxy injection and CFRP jacketing in repair and strengthening of the beamcolumn joints under cyclic loading.

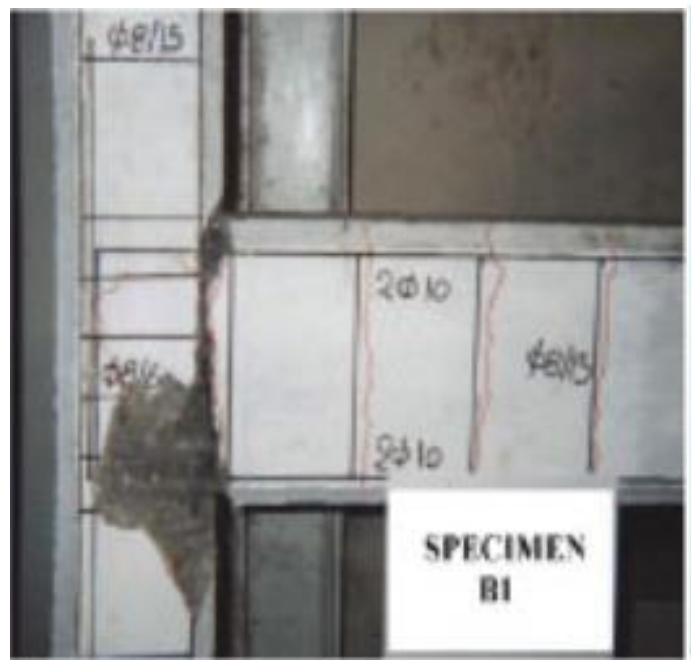

(a)

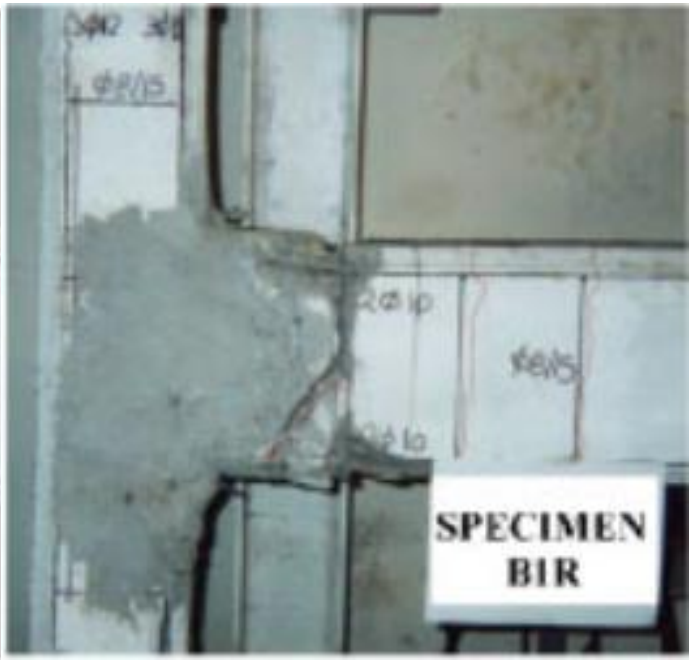

(b)

Figure 2-17: Failure of the beam-column joint under cyclic loading a) Failure occurance at the joint before repair b) Failure occurance outside of the joint after repair with epoxy injection (Karayannis and Sirkelis, 2008)

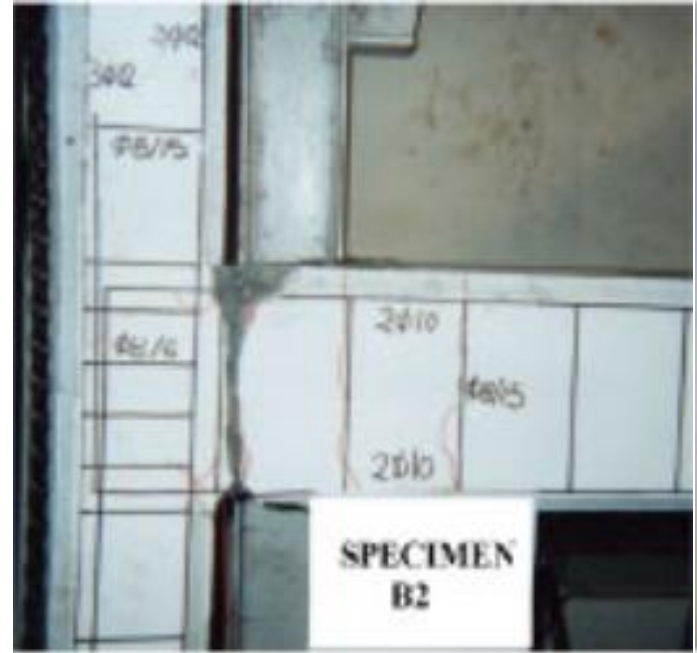

(a)

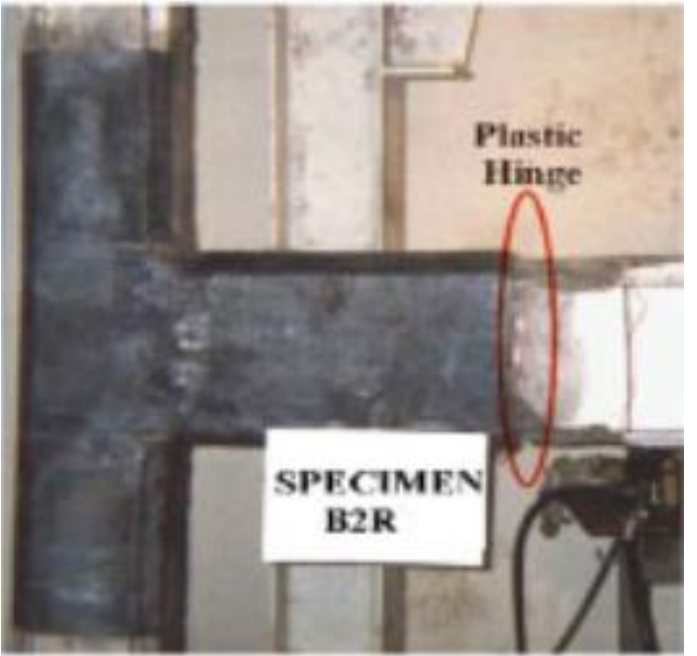

(b)

Figure 2-18: Failure of the bam-column joint under cyclic loading a) Failure occurance at the joint before repair b) Failure occurance outside of the strengthened area after repair with epoxy injectiona and CFRP jacketing (Karayannis and Sirkelis, 2008) 
Sadjadi et al. (2009) investigated the effectiveness of the GFRP laminate in repairing cracked RC members subjected to monotonic tensile loading and cyclic flexure under hydrostatic water pressure. For this purpose, $\mathrm{RC}$ members were tested under monotonic tension and cyclic flexure and leakage test was performed. Next, specimens were repaired externally with GFRP laminate and subjected to monotonic tensile loading and cyclic flexure under hydrostatic water pressure. No failure was observed in the specimen repaired with GFRP laminate under monotonic tensile loading in exposure to water pressure. However, applying repeated cycles of flexure resulted in failure of the GFRP laminate and leakage of the specimen. It was found by Sadjadi et al. (2009) that GFRP laminate had a great potential in water proofing and repair of cracked RC members subjected to monotonic tension, while the performance of GFRP laminate in exposure to cyclic flexure was found to be disputable.

Nikopour and Nehdi (2011) performed a study on the repair of shear deficient RC beams using hybrid unidirectional FRP sheets and epoxy injection. A total number of 6 full-scale RC beams with cross section of $150 \mathrm{~mm}$ width and $250 \mathrm{~mm}$ height and having the length of $2438 \mathrm{~mm}$ were fabricated. Beams were designed as over-reinforced in flexure to make sure the ultimate shear capacity was reached before failure occurred in flexure. The experimental program was performed in two phases. In the first phase, 4 shear deficient beams having hybrid unidirectional side bonded FRP sheets and 2 control beams as control sample were tested under cyclic loading. CFRP sheets, and combinations of carbon-aramid, carbon-glass, and glass-glass fiber reinforced polymer sheets were used as external wrapping of 4 shear deficient beams to be tested under cyclic loading. In the next phase, 3 of the beams were repaired using epoxy injection and also simultaneous application of epoxy injection and carbon fiber reinforced polymer (CFRP) sheets and retested under fourpoint static loading. Figure 2-19 shows the procedure of repairing the $\mathrm{RC}$ beams with low-pressure 
epoxy injection. It was found by Nikopour and Nehdi (2011) that hybrid application of FRP sheets increased the shear capacity of the beams compared to unidirectional CFRP repaired beams. Cyclic loading and fatigue resulted in formation of cracks and weakening the bond between the concrete surface and externally bonded FRP sheet. Moreover, simultaneous application of crack epoxy injection and FRP external wrapping was found to be effective in terms of improving the ultimate strength and stiffness of the RC beams.

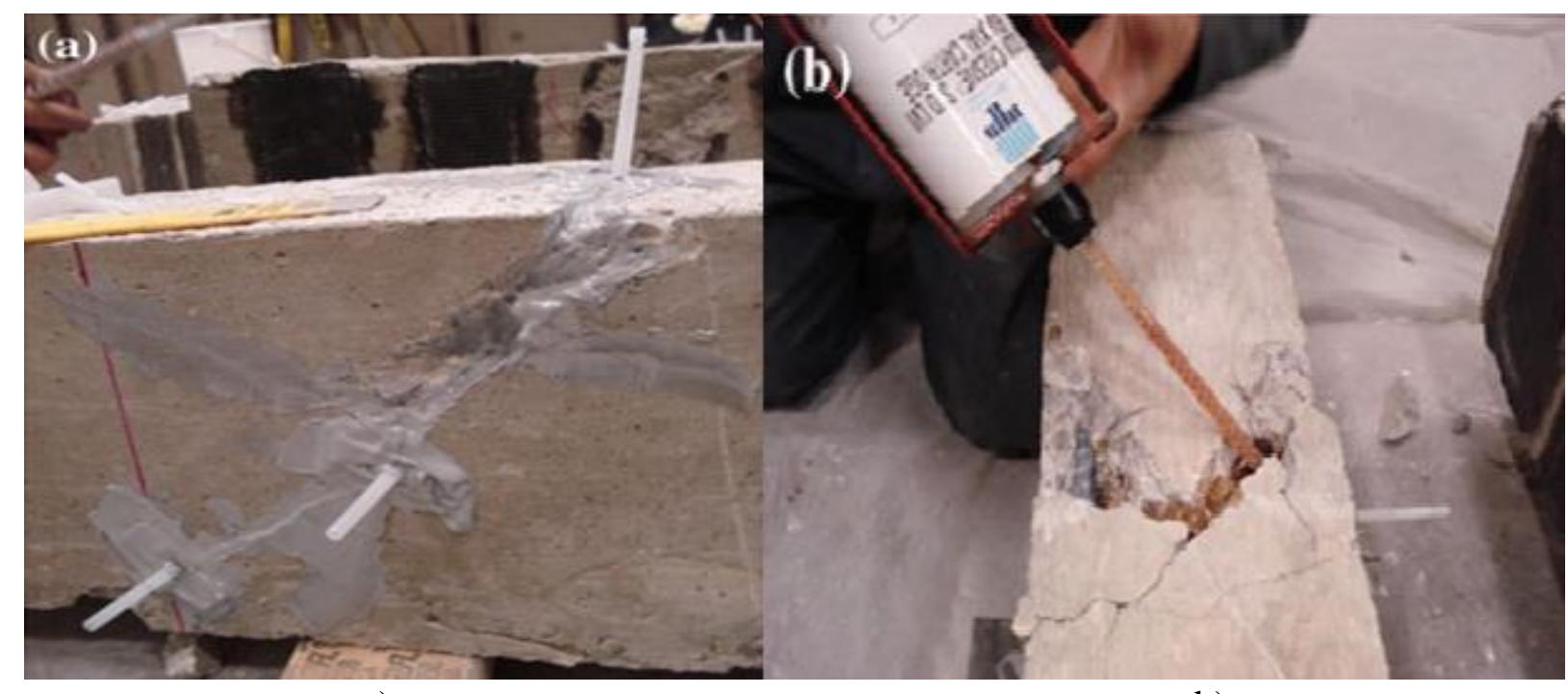

a)

b)

Figure 2-19: Repair of RC beams using low-pressure epoxy injection a) Sealing the cracked surface and installing the injection ports b) Low-pressure epoxy injection

(Nikopour and Nehdi, 2011)

In another study performed by Torabi and Maheri (2016) effectiveness of section enlargement using stiffened steel plates in repairing full-scale external beam-column joints was investigated under quasi-static cyclic loading. For this purpose, an external full-scale beam-column joint was fabricated and tested under lateral cyclic loading. In the next step, the beam-column joint was repaired using joint enlargement method. Joint cracks were epoxy injected followed by filling the damaged area by pouring fresh concrete. Next, stiffened steel plate was placed and fixed at joint location. The retrofitted specimen was tested up to failure under lateral cyclic loading. Seismic parameters such as strength, ductility, energy absorption, and stiffness were recorded and analyzed 
to determine the effectiveness of the repair program. It was found by Torabi and Maheri (2016) that repairing the beam-column joint by incorporating stiffened steel plate under lateral cyclic loading improved the strength by $35 \%$, reduced the ductility, and increased the stiffness of the joint. Moreover, plastic hinge relocated to the beam member away from the joint in the repaired joint. Failure location and formation of plastic hinge in the specimen before and after applying the repair is illustrated in Figure 2-20. The study confirmed applicability of the stiffened steel plate repair technique in strengthening of the beam-column joints under lateral cyclic loading.

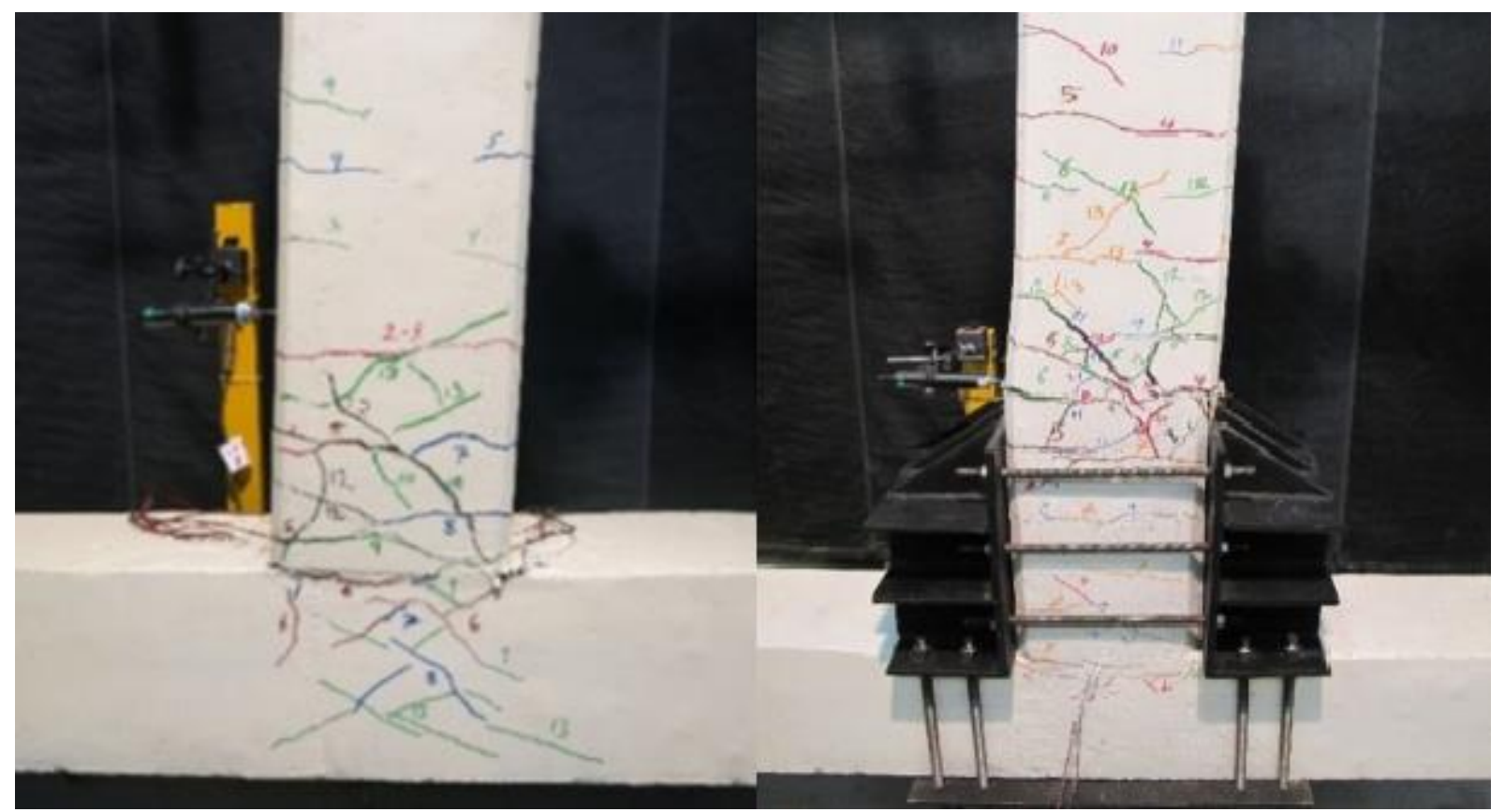

(a)

(b)

Figure 2-20: Failure of the beam-column joint under lateral cyclic loading a) Formation of plastic hinge at the joint location $b$ ) Formation of the plastic hinge in the beam member away from the joint (Torabi and Maheri, 2016)

Anwar et al. (2009) investigated the effectiveness of ECC and CFRP sheets in repairing concrete samples in flexure. For this purpose, 21 plain concrete samples measuring $100 \mathrm{~mm}$ width $\mathrm{x} 100$ mm depth $\times 400 \mathrm{~mm}$ length were cast and tested under four-point loading condition. All the samples contained pre-defined artificial crack at mid-span with depth of $50 \mathrm{~mm}$ and width of 2 mm. Cracked concrete samples were filled with epoxy injection and repaired with layer of ECC or 
simultaneous application of ECC layer and CFRP sheet. ECC repair with various thicknesses of $10 \mathrm{~mm}, 30 \mathrm{~mm}$, and $50 \mathrm{~mm}$ was used on the tension side of the samples along the longitudinal direction with total length of $380 \mathrm{~mm}$. Specimens were tested under four-point loading condition and structural performance of the repaired specimens was compared to the control sample (concrete sample without cracking). It was observed that higher thicknesses of ECC repair layer enhanced both load capacity and ductility of the damaged concrete samples. Use of CFRP sheet resulted in the same flexural strength, while changing the failure mode from interfacial debonding to shear failure. Combined application of ECC and CFRP sheet resulted in improving the section load capacity and ductility along with minimizing the interfacial debonding of the repair layer. The study confirmed the effectiveness of combined application of ECC and CFRP sheet in repairing concrete samples under flexural loading.

Sing and Sivasubramanian (2013) performed a study on the effect of strengthening RC beams with ECC under flexural loading. A total number of 12 half-scale RC beams were fabricated and tested under four-point loading. Two strengthening methods were used including strengthening partially at plastic hinge region with ECC and strengthening with ECC layers on the entire tension side, as shown in Figure 2-21 and Figure 2-22.

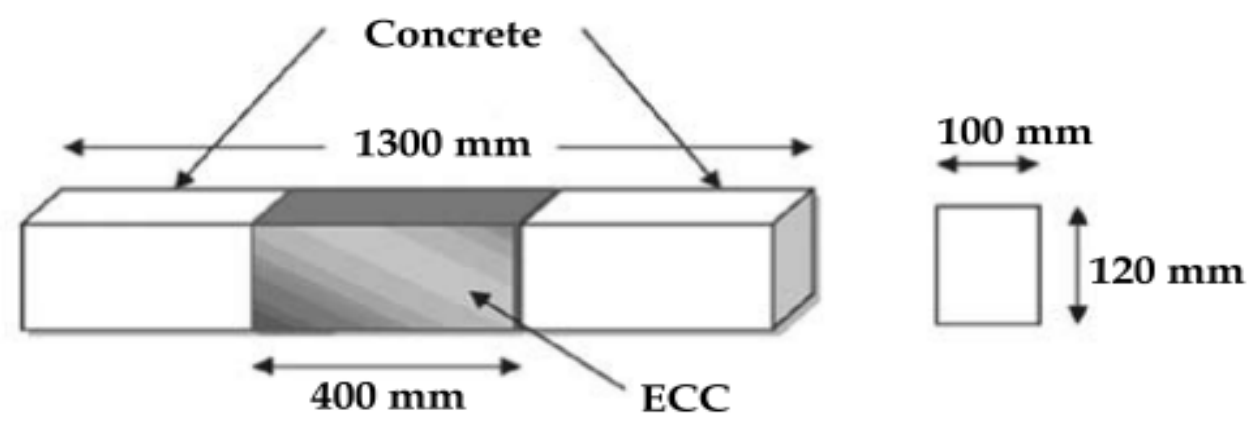

Figure 2-21: Strengthening with ECC at plastic hinge region (Sing and Sivasubramanian, 2013) 


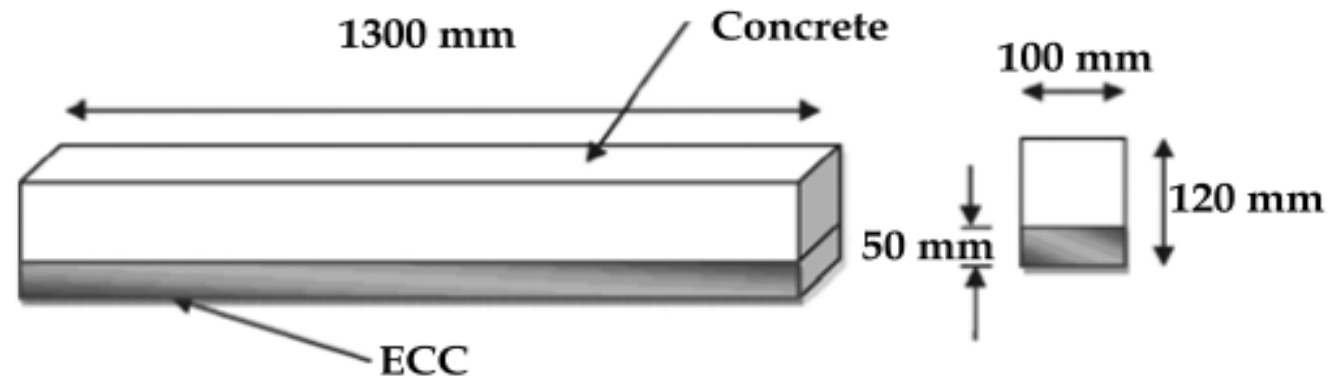

Figure 2-22: Strengthening with ECC layer on the tension side (Sing and Sivasubramanian, 2013)

All the specimens had the same cross-sectional dimension of $100 \mathrm{~mm}$ width and $120 \mathrm{~mm}$ depth (including the ECC layer) along with a total length of $1300 \mathrm{~mm}$. RC beams were designed as overreinforced and under-reinforced to control the failure mode of the beams. Six RC beams were strengthened with various lengths of $200 \mathrm{~mm}, 400 \mathrm{~mm}$, and $600 \mathrm{~mm}$ of ECC at plastic hinge region. Also, four RC beams were strengthened with $50 \mathrm{~mm}$ and $70 \mathrm{~mm}$ thick ECC layers on the tension side along with 2 RC beams which were used as control samples. Specimens were tested under four-point loading and experimental results were recorded and compared to the control samples. It was found by Sing and Sivasubramanian (2013) that over-reinforced and under-reinforced RC beams strengthened with ECC at plastic hinge regions exhibited load capacity of 2.85- 6.34 times and 1.55- 4.83 times more than corresponding control samples. Moreover, over-reinforced and under-reinforced RC beams strengthened with ECC layer on the tension side showed load capacity 5.7- 8.45 times and 5.37- 8.31 times more than the corresponding control samples. The study confirmed the effectiveness of using ECC layer on the tension side and in the plastic hinge regions in strengthening of RC beams in flexure.

Kim et al. (2013) performed investigation on strengthening of RC beams by incorporating strain hardening cementitious composite (SHCC) and high-strength reinforcing steel (HSRS) bars under flexural loading. For this purpose, four RC beams were fabricated and tested under four-point 
loading condition. Shear reinforcement was provided for the beam to prevent shear failure. Beam specimens had cross-section dimensions of $300 \mathrm{~mm}$ width by $500 \mathrm{~mm}$ height along with total length of $3400 \mathrm{~mm}$. Combination of fly ash and ground granulated blast furnace slag (GGBS) along with Portland cement was used as cementitious materials in the SHCC mix design. Three RC beams were strengthened with SHCC layer on the tension face and HSRS bars including one specimen strengthened with $60 \mathrm{~mm}$ thick layer of SHCC, one specimen strengthened with $60 \mathrm{~mm}$ thick layer of SHCC and 3 HSRS bars, and third specimen strengthened with $60 \mathrm{~mm}$ thick layer of SHCC along with 5 HSRS bars. One of the beam specimens was also used as control sample. Beam specimens were tested under four-point loading condition and experimental results were recorded and compared to the control sample. It was observed that crack width of the beam specimens could be controlled by applying the SHCC layer on the tension side of the beam. The study confirmed the applicability of SHCC and HSRS bars in strengthening of RC beams in flexure by exhibiting higher load bearing capacity compared to the control sample.

Wang and Zheng (2015) investigated the combined application of BFRP grid and ECC in strengthening of RC beams in flexure. For this purpose, four RC beam specimens with cross section dimensions of 200 width and $300 \mathrm{~mm}$ height along with a total length of $1800 \mathrm{~mm}$ were fabricated and tested under four-point loading condition. Thickness of the BFRP grid was selected as the only variable parameter in that experimental program. Strengthening system included applying various thicknesses of BFRP grid $(1 \mathrm{~mm}, 3 \mathrm{~mm}$, and $5 \mathrm{~mm})$ along with casting $30 \mathrm{~mm}$ ECC layer on the tension of the beam specimens in longitudinal direction. One RC beams was also used as control sample. Beam specimens were tested under four-point loading and loaddisplacement response, strain development, and failure modes of the specimens were discussed. It was observed that the governing failure mode in control sample was concrete crushing, while 
tensile rupture of the BFRP grid along with concrete crushing or debonding of strengthening layer was identified as failure mode of strengthened beam specimens, as shown in Figure 2-23. It was found by Wang and Zheng (2015) that strengthened beam specimens showed considerably higher flexural stiffness and load bearing capacity compared to control samples. The study confirmed the effectiveness of the combined application of BFRP grid and ECC in flexural strengthening of the RC beams.

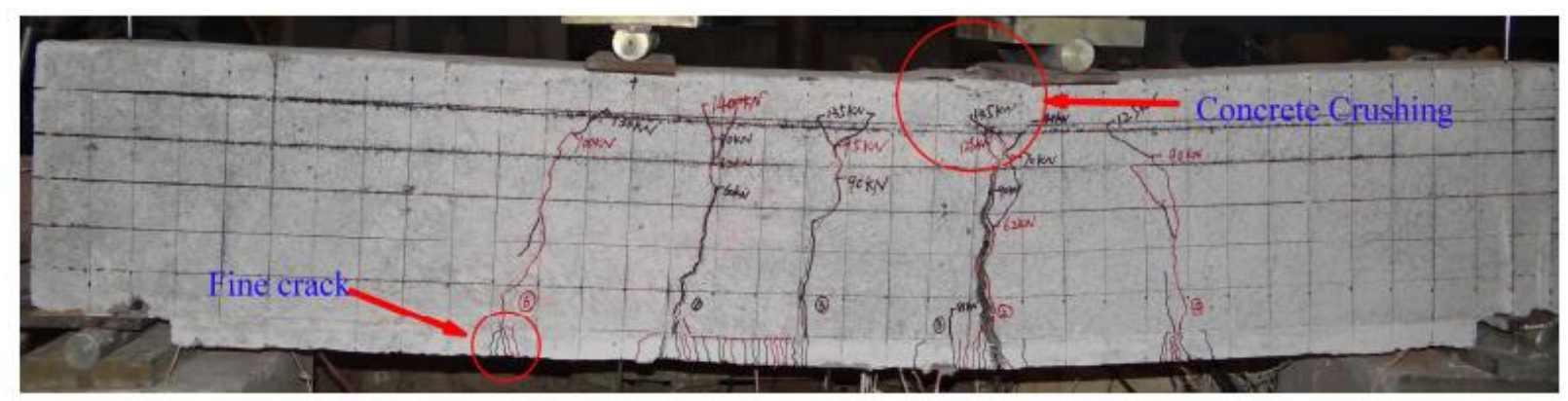

(a)

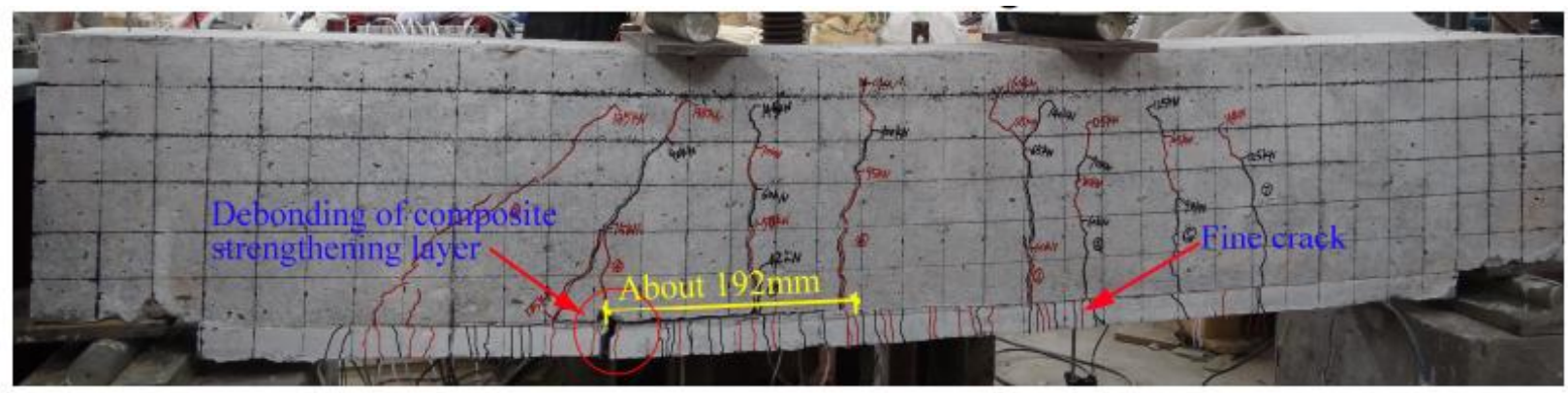

(b)

Figure 2-23: Failure mode of the strengthened RC beams with BFRP grid and ECC a) BFRP tensile rupture and concrete crushing b) BFRP tensile rupture and debonding of the strengthening layer (Wang and Zheng, 2015)

Zhongfeng and Wenwei (2016) investigated the effectiveness of FRP textile with ECC matrix in strengthening of $\mathrm{RC}$ circular columns under axial compression loading. RC circular columns specimens were fabricated and tested under static axial compression up to failure and experimental results were recorded and compared to the control samples. It was observed that all strengthened specimens experienced rupture of the FRP textile as the governing failure mode. It was found by 
Zhongfeng and Wenwei (2016) that column specimens strengthened with FRP textile with ECC matrix exhibited higher load bearing capacity compared to the columns samples without strengthening.

Hung and Chen (2016) investigated the effectiveness of ECC jacketing in strengthening of shear deficient RC cantilever beams under cyclic loading. A total number of $5 \mathrm{RC}$ cantilever beams were fabricated and tested under cyclic loading including five beam specimens, strengthened with ECC jacketing, along with one control sample without jacketing. Beam specimens had similar crosssection dimensions of $250 \mathrm{~mm}$ width by $350 \mathrm{~mm}$ depth and overall length of $1200 \mathrm{~mm}$. Strengthening method involved U-shape (three-sided) ECC jacketing with a constant thickness of $40 \mathrm{~mm}$, as shown in Figure 2-24. Test variables included use of ECC or mortar as the jacketing material, presence of reinforcement in the jacketing layer, and use of welded steel bar or wire mesh as the reinforcement of the jacketing layer. Strengthened beam specimens were tested under cyclic loading and the structural performance was evaluated by analyzing hysteretic loops, damage patterns, energy dissipation capacities, strain development in the rebars, shear distortions, and failure modes. It was observed by Hung and Chen (2016) that ECC jacketing without steel reinforcement was capable of improving the cyclic behavior of the RC cantilever beams. Reinforcing the ECC jacketing layer with steel mesh further improved the structural performance of the strengthened beam specimen. The study confirmed the effectiveness of ECC jacketing with a single layer of steel mesh in strengthening of the RC cantilever beams under cyclic loading. 


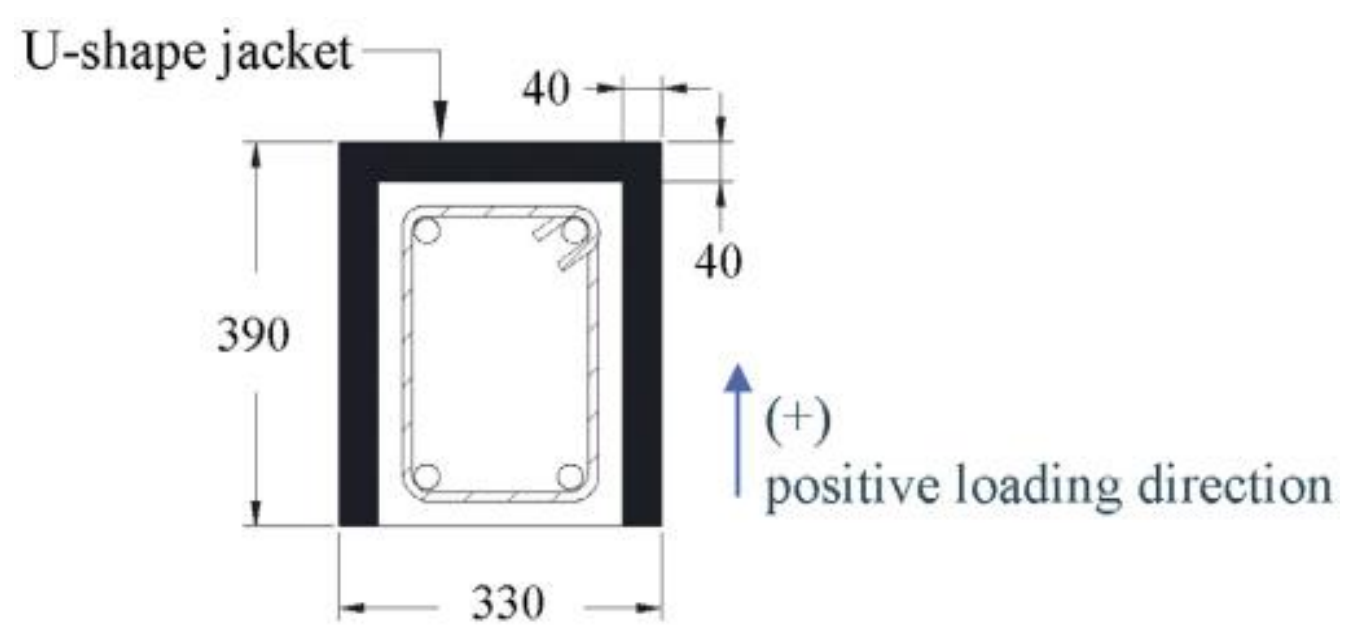

Figure 2-24: Cross-section of the strengthened RC beams with $40 \mathrm{~mm}$ thick U-shape ECC jacketing (Hung and Chen, 2016)

Deng et al. (2018) investigated the effectiveness of ECC and ferrocement jacketing in shear strengthening of short RC square columns under lateral cyclic loading. For this purpose, seven half-scale RC square columns were fabricated with cross-section dimensions of $250 \mathrm{~mm}$ by 250 $\mathrm{mm}$ and overall height of $600 \mathrm{~mm}$. Two column specimens were used as the control samples, while one specimen was strengthened with ferrocement jacketing, one specimen strengthened with ECC jacketing without reinforcement, and three column samples were strengthened with ECC jacketing containing bar mesh as the reinforcement. Column specimens were tested under lateral cyclic loading and structural performance was assessed by analyzing hysteresis responses, deformation capacity, stiffness degradation, and energy dissipation capacity. It was observed that control samples and column strengthened with ferrocement jacketing failed in a brittle manner, while ECC jacketing strengthened specimens showed much more ductile behavior as well as improvement in plastic deformation ability and energy dissipation capacity due to strain hardening ability of ECC. Shear deformation and shear strength of the column specimens were improved by application of external ECC jacketing. The study confirmed the effectiveness of ECC jacketing in shear strengthening of the RC square columns under lateral cyclic loading. 
Shriram et al. (2018) performed an investigation on strengthening of RC beams using ECC in shear and flexure. A total number of $4 \mathrm{RC}$ beams were fabricated and tested under four-point loading. Beam specimens had similar cross section dimensions of $100 \mathrm{~mm}$ width and $150 \mathrm{~mm}$ height along with an overall length of $1000 \mathrm{~mm}$. Polyester fibers content of $1 \%$ by volume was used in ECC mix. Three methods were used to strengthen the RC beams including embedment of $25 \mathrm{~mm}$ ECC layer as the concrete cover on the tension side of the beam, applying the $25 \mathrm{~mm}$ ECC layer on the tension face of the beam, and applying the $25 \mathrm{~mm}$ ECC layer on the tension side as well as both sides of the beam. One of the beams was used as the control sample without strengthening, as shown in Figure 2-25. Beam specimens were tested in flexure under four-point loading condition.
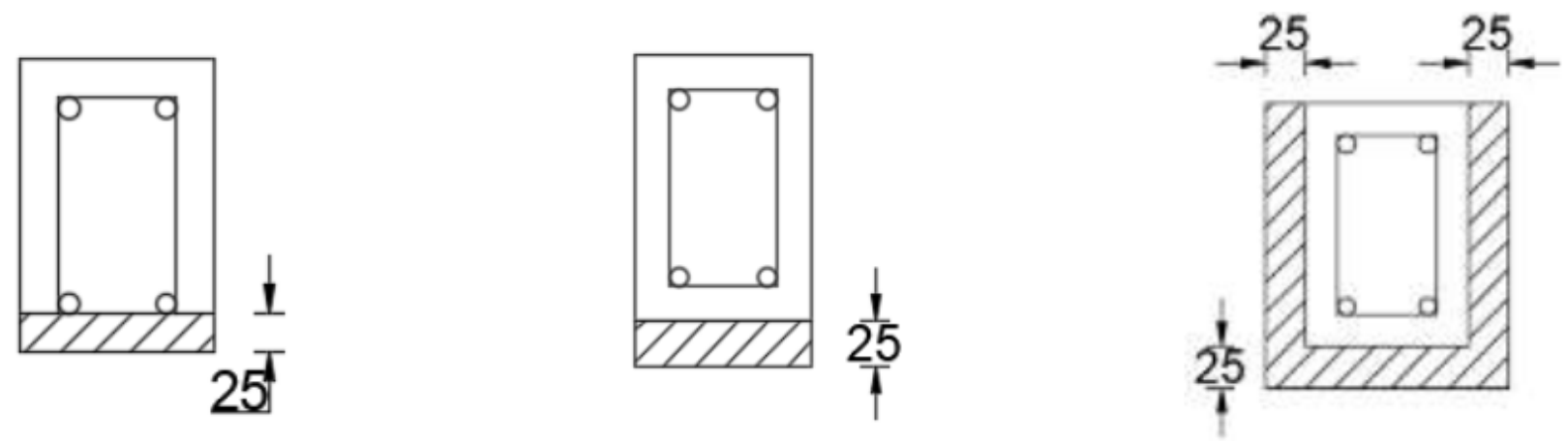

Figure 2-25: Strengthening methods of RC beam specimens with ECC layer in flexure

(Shriram et al., 2018)

It was concluded by Shriram et al. (2018) that RC beams strengthened with ECC layer on the tension face and both sides showed the highest load capacity, more ductility, and energy absorption in comparison with other specimens. Moreover, strengthening of the beam specimens with embedment of the ECC layer on the tension side did not make any significant improvement in term of strength characteristics. The study confirmed the effectiveness of strengthening RCC beam with ECC on three faces (tension face and both sides) in flexure.

Yang et al. (2018) investigated the effectiveness of CFRP grid with ECC matrix as an external overlay in strengthening RC beams in flexure. A total number of $15 \mathrm{RC}$ beams were fabricated 
including 3 control sample without strengthening, measuring $200 \mathrm{~mm}$ width, 150 depth, and overall length of $1800 \mathrm{~mm}$, along with $12 \mathrm{RC}$ beam strengthened with $20 \mathrm{~mm}$ thick external overlay composed of CFRP grid and ECC matrix. Experimental variables included longitudinal tensile reinforcement ratio ranging between $0.79 \%$ and $1.74 \%$, use of different materials as mortar matrix, ECC or epoxy mortar, various stiffness levels of CFRP grids, and installation method of the strengthening overlay, cast in place or pre-fabricated. Beam specimens were tested under fourpoint loading condition up to failure and experimental results were recorded and compared in terms of load-deflection responses, crack patterns, failure modes and strain development in concrete, rebars, and CFRP grids. It was observed that use of ECC or epoxy mortar as the matric material and bonding adhesive of CFRP grid did not completely prevent the debonding failure of the strengthening overlay system. However, use of ECC as the matrix and bonging adhesive of the CFRP grid resulted in a more ductile failure compared to epoxy mortar, due to multiple cracking behavior of the ECC. It was found by Yang et al. (2018) that simultaneous use of pre-fabricated ECC matrix for the CFRP grid along with epoxy resin for proper bonding of the strengthening overlay system to the tension face of the RC beam solved the problem of debonding failure. The study suggested ECC could be an ideal choice as cementitious matrix for CFRP grid in case of an external overlay system in strengthening of RC beams under flexural loading.

AL-Gemeel and zhuge (2018) performed a study on the application of basalt fiber textile with ECC matrix as a strengthening system for confining RC square columns under axial loading. Square RC columns were designed as short columns with cross-sectional dimension of $60 \mathrm{~mm}$ by $60 \mathrm{~mm}$ along with overall length of $200 \mathrm{~mm}$. 18 RC columns were prepared and tested including 3 columns as control samples, 3 column specimen strengthened with ECC, 9 columns specimens strengthened with ECC matrix and BFRP grids with various spacings of $10 \mathrm{~mm}, 25 \mathrm{~mm}$, and 50 
$\mathrm{mm}$, as well as 3 columns strengthened with cement mortar as the matrix and BFRP grid with spacing of $25 \mathrm{~mm}$. Basalt fiber mesh types and confined column details are shown in Figure 2-26. Experimental variables included the basalt fiber textile (basalt fiber grid spacing) and use of ECC or cement mortar as the matrix for the BFRP grid. Compressive strength test was performed on all RC column specimens according to ASTM standards up to failure. Experimental results were recorded ad compared to the control samples. It was observed that column specimens strengthened using just ECC and using basalt fiber textile with ECC matrix exhibited significantly higher load bearing capacity compared to columns without strengthening and column specimens strengthened with basalt fiber textile with cement mortar matrix. The study confirmed the effectiveness of ECC alone as well as basalt fiber textile with ECC matrix in confining non-circular RC columns under axial compressive loading.

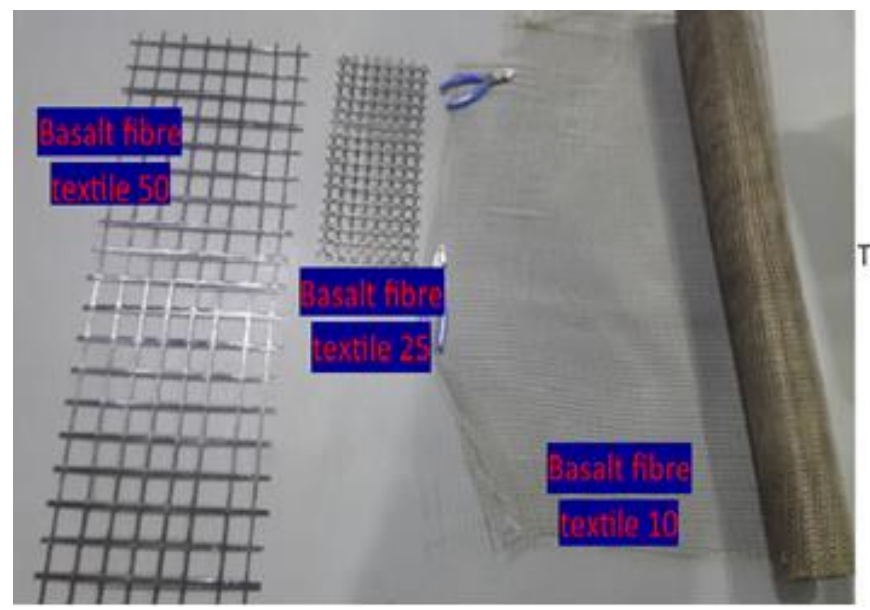

(a)

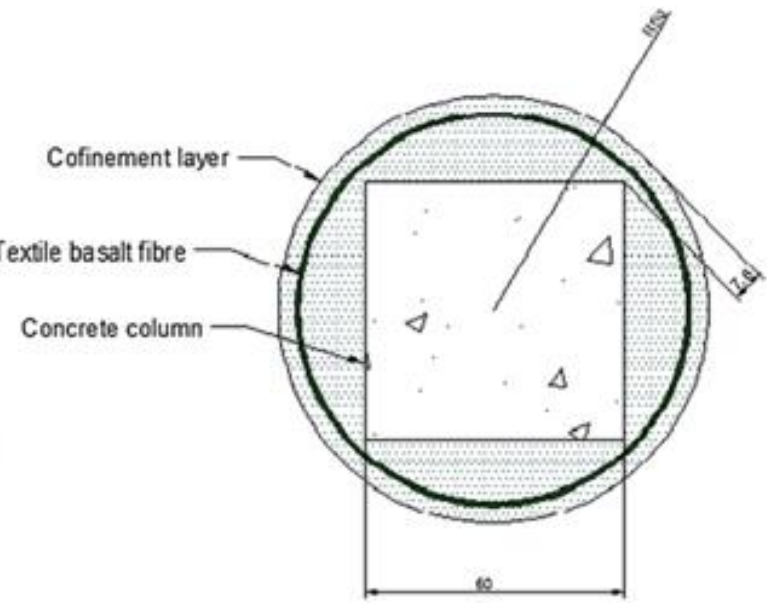

(b)

Figure 2-26: Column strengthening with ECC and BFRP textile a) Basalt fiber mesh textile b) Confined column details (AL-Gemeel and zhuge, 2018)

Wang et al. (2019) investigated the performance of ECC in strengthening of shear deficient RC beams under four-point loading. A total number of 12 shear deficient RC beams were fabricated and tested under four-point loading. All the beams had similar cross section dimensions of 150 $\mathrm{mm}$ width by $300 \mathrm{~mm}$ height along with total length of $2100 \mathrm{~mm}$. The beams were designed as 
over-reinforced sections in flexure and without shear reinforcement in the middle part to ensure reaching the ultimate shear capacity before failure in flexure. Specimens were strengthened by spraying ECC to the both sides of beams with varying thickness of $20 \mathrm{~mm}$ and $40 \mathrm{~mm}$. The variable parameters in the test were thickness of the ECC layer, longitudinal reinforcement ratio, and shear span to depth ratio. The performance of the specimens was described based on load versus midspan deflection, shear stress and strain, and failure mode of the specimens. It was found by Wang et al. (2019) that RC beams strengthened with ECC repair layer exhibited higher shear capacity by $89 \%$ compared to the concrete samples. The study concluded the effectiveness of ECC layer in shear strengthening of RC beams.

Hou et al. (2020) proposed the application of combined Basalt fiber reinforced polymer (BFRP) bars and ECC in strengthening of RC beams under flexure. A total number of $6 \mathrm{RC}$ beams were fabricated and tested under four-point loading condition. The RC beams had similar cross section of $200 \mathrm{~mm}$ width and $300 \mathrm{~mm}$ height as well as overall length of $1900 \mathrm{~mm}$. BFRC bars reinforcement ratio (ranging from $0.94 \%$ to $1.41 \%$ ), method of installation (pre-fabricated or castin-place), and use of steel bolts as end anchorage system were considered as variables in the experimental program. The strengthening layer was designed to have the same length and width as RC beams along with a thickness of $30 \mathrm{~mm}$. One RC beam was used as the control sample and 5 other RC beams were strengthened with ECC and BFRP bars and tested under four-point loading conditions. Structural performance of the specimens was judged based on load versus deflection response, strain development, crack patterns, and failure modes. Two modes of failure were observed in concrete specimens strengthened with ECC and BFRP bars including the rupture of the BFRP bars as well as debonding of the concrete cover and the repair layer from concrete substrate, depending on the BFRP bars reinforcement ratio. Use of steel bolts as end anchorage 
system improved the bond between the concrete specimen and repair layer, allowing better performance of the strengthening system. It was found by Hou et al. (2020) that combined application of BFRP bars and ECC as strengthening system improved the load bearing capacity of $\mathrm{RC}$ beams in flexure.

\section{2-4 Review conclusions}

Extensive research works have been performed on material properties of ECC for tailoring improved mechanical and durability properties. ECC has also been used for several real-world structural applications over the years. A number of investigations have been conducted on the application of ECC in repair and strengthening of RC members under various loading conditions. However, there is no research available in the literature focusing on the repair of liquid containing structures using ECC. This study is warranted and will investigate the applicability of ECC in repairing liquid containing structures by evaluating the ECC performance in repairing NC panels subjected to direct tensile loading under hydrostatic water pressure. Moreover, ECC efficiency is compared to currently used repair materials in industry including high-strength epoxy and FRP laminates in terms of strength, cracking and leakage behavior, and self-healing ability. The findings of this research are directly applicable to structures that are designed under serviceability limit states including liquid containing structures, in which crack control, water tightness and leakage behavior are of prime importance. 


\section{Chapter Three: Experimental Program}

\section{3-0 Introduction}

The experimental program has been devoted to the investigation of repair and self-healing of tension induced cracks in RC panels by incorporating high-performance materials including highstrength low viscosity epoxy, glass fiber reinforced polymer (GFRP) laminates, engineered cementitious composites (ECC) using slag, and engineered cementitious composites (ECC) using fly ash. A total number of 4 reinforced concrete panels made with normal concrete (NC) are tested under direct tensile loading and load, strain, crack width, and leakage data are recorded. In the next step, NC concrete panels are repaired with above mentioned materials and performance of each repair material is evaluated in terms of the load at which leakage initiated, crack width and the corresponding leakage rate under different water pressures. This chapter describes the geometrical dimensions of the concrete panels, material properties, experimental setup, and testing procedure.

\section{3-1 Geometric dimensions of specimens and reinforcement details}

The experimental program has been conducted to study the cracking and leakage behavior of the RC panels. A total number of $4 \mathrm{RC}$ panels made with $\mathrm{NC}$ were constructed, tested, and repaired with high-strength epoxy, GFRP laminate, ECC slag, and ECC fly ash. Each specimen measured $1500 \mathrm{~mm}$ (Length) $\times 400 \mathrm{~mm}($ Width $) \times 250 \mathrm{~mm}($ Depth $)$ representing the wall or slab segment of a tank and direct tensile loading is applied on the specimens to simulate the ring force (hoop tension) in the tank wall resulted from hydro-static pressure of water inside the tank. Repair program is applied on the top surface of the NC panels which is in contact with water pressure 
chambers to simulate repairing internal surface of a cracked wall segment of a tank which is exposed to hydrostatic water pressure. Simulating the cracking and leakage behavior, and selfhealing of tension-induced cracks in a water tank may arise a number of challenges. First, water chamber should be mounted on top of the NC panels, tightened and sealed properly, to ensure exposing the cracked section to pressurized water, while it would not contribute in carrying the direct tensile load. Resisting any portion of the tensile load by water chamber may negatively affect the cracking behavior of the NC panel. Another challenge that may be encountered is exposing the full length of crack to pressurized water. In case of partial exposure of the crack length to pressurized water, water penetrates to the crack and leak from the bottom surface of the panel through a diagonal pathway, which is longer than the depth of panel, resulting in underestimating the leakage rate of NC panel. Lastly, providing pure tension loading condition poses another challenge. Presence of eccentricity in any direction jeopardizes the assumption of uniform tensile stress distribution over the NC panel cross-section. This may result in lifting up the NC panel from the support on one side, uneven crack width over the NC panel cross-section, or in more severe cases, development of compression zone in some part of the cracked section. However, a unique test setup has been designed to reproduce the cracking, leakage, and self-healing behavior of tension cracks in water tanks while addressing the challenges. Figures 3.1 and 3.2 show the plan and side view of the test setup, respectively. Two manual hydraulic jacks are installed on both sides of the sample to apply the monotonic tension loading. Furthermore, two steel hollow structural sections (HSS) are placed on north and south sides of the sample to transfer the applied tension loading to the rebar. Two large solid round bars are also placed on the right and left sides of the sample for load transmission from the hydraulic jacks to HSS. A single 35-M rebar is placed at the center of the section to prevent any eccentricity during the tension loading stage. The rebar 
length is $3000 \mathrm{~mm}$ with an embedded part of $1500 \mathrm{~mm}$ inside the sample while extruded out of the RC panel $750 \mathrm{~mm}$ on each of the north and south ends, by passing through the holes fabricated in the middle of the HSS. Both ends of the rebar are then fixed by welding the nuts which are placed at the back of the HSS. Moreover, in order to provide a free sliding boundary condition, specimens are supported on rollers at both ends in the direction of tensile loading, as shown in Figure 3.2.

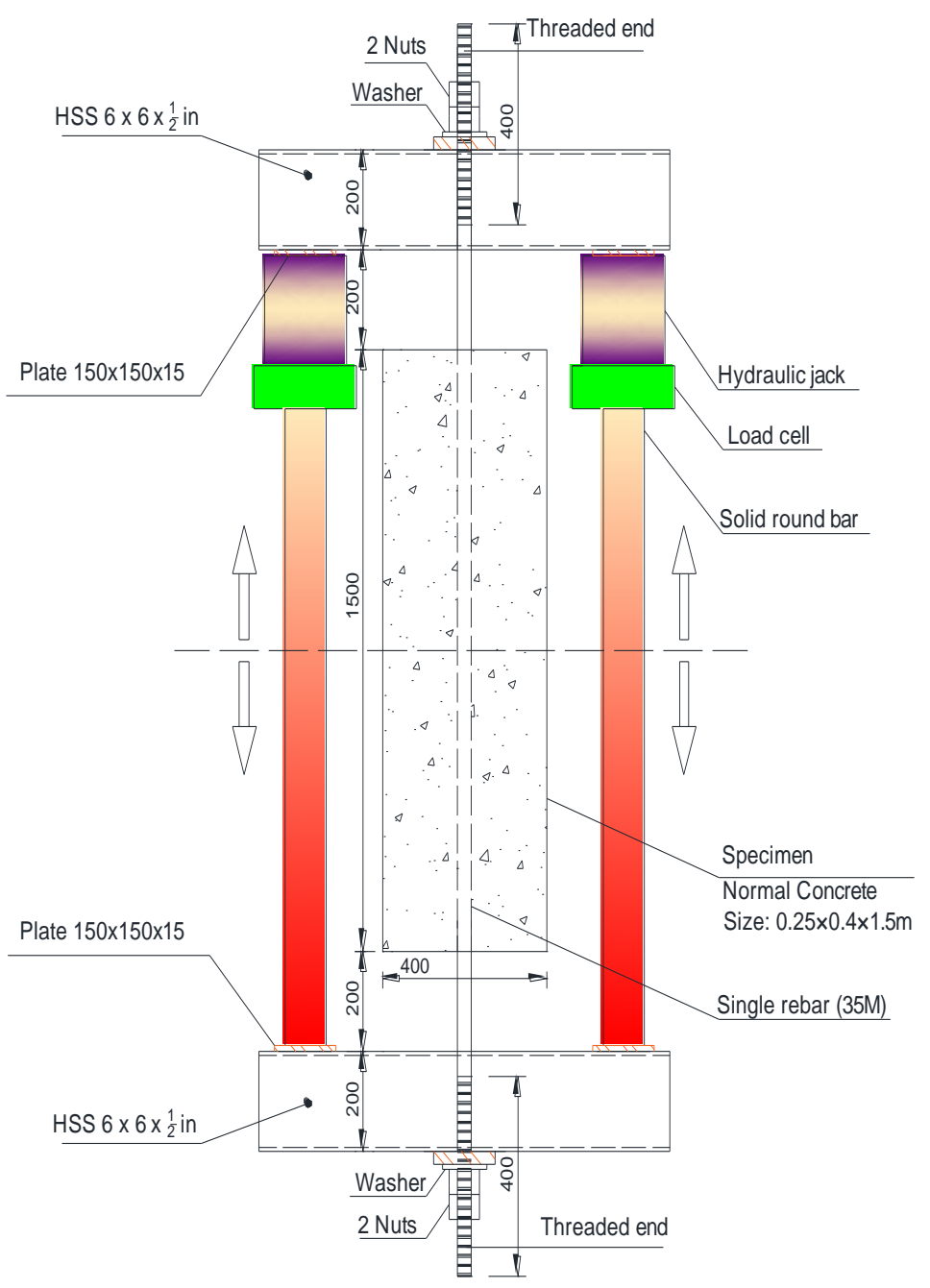

Figure 3.1: Plan view of the test setup 


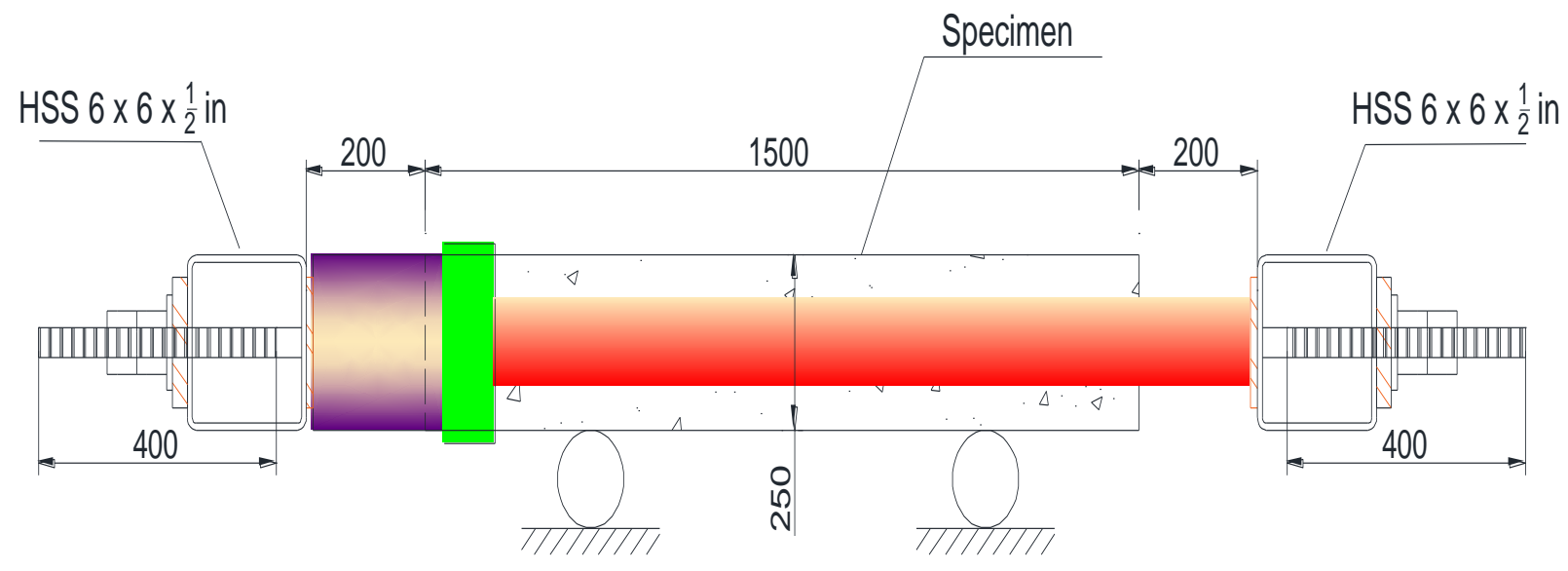

Figure 3.2: Side view of the test setup

In order to inspect the leakage behavior of the RC panel through visual observation, installation of the water chamber is required to maintain the desired water pressure over the cracked region. The water pressure chamber is designed and fabricated of $5 \mathrm{~mm}$ steel plate and illustrated in Figure 3.3. The water chamber is placed in the middle of the section $(750 \mathrm{~mm}$ from each end), where the major crack forms in the specimens. A $25 \mathrm{~mm}$ thick layer of gum rubber pad is placed between the water pressure chamber and concrete surface in order to provide flexibility for free deformation of the RC panel along the tension loading axis. A water-resistant silicone is used to seal the entire contact area between rubber pad and concrete surface as well as rubber pad and water chamber. The water chamber mounted on the specimen along with the gum rubber pad is shown in Figure 3.4 .

To ensure a full sealing condition under high water pressures, water chambers are clamped to the RC panel using two channels on top of the chambers as well as two U-shape channels underneath the specimen, while tightened together using $20 \mathrm{~mm}$ diameter steel rods. With the intention of major crack formation in predetermined location, where the water chamber is installed, a transverse notch with the dimensions of $40 \mathrm{~mm}$ deep and $10 \mathrm{~mm}$ thick is created in the middle section at the bottom surface of the RC panels. No notch is placed on the top surface of the 
specimens, since it may interfere with normal functioning of the repair materials. In favor of preventing the leakage from the sides of the sample, two pieces of waterproof membrane sheets are attached to the sides of the specimen using a water-resistant adhesive. By doing so, the water travels through the crack over the full-depth of the specimen leaking from the bottom which simulates the leakage through the cracked wall section of a tank.

The city water system is connected to the inlet of the water chamber. The test is performed under different water pressures and there is need to maintain a constant water pressure over a specific period of time. However, the leakage from the bottom face results in dropping the water pressure during the test. For this reason, a pressure regulator as well as a pressure gauge are installed to control and monitor the water pressure inside the water chamber.
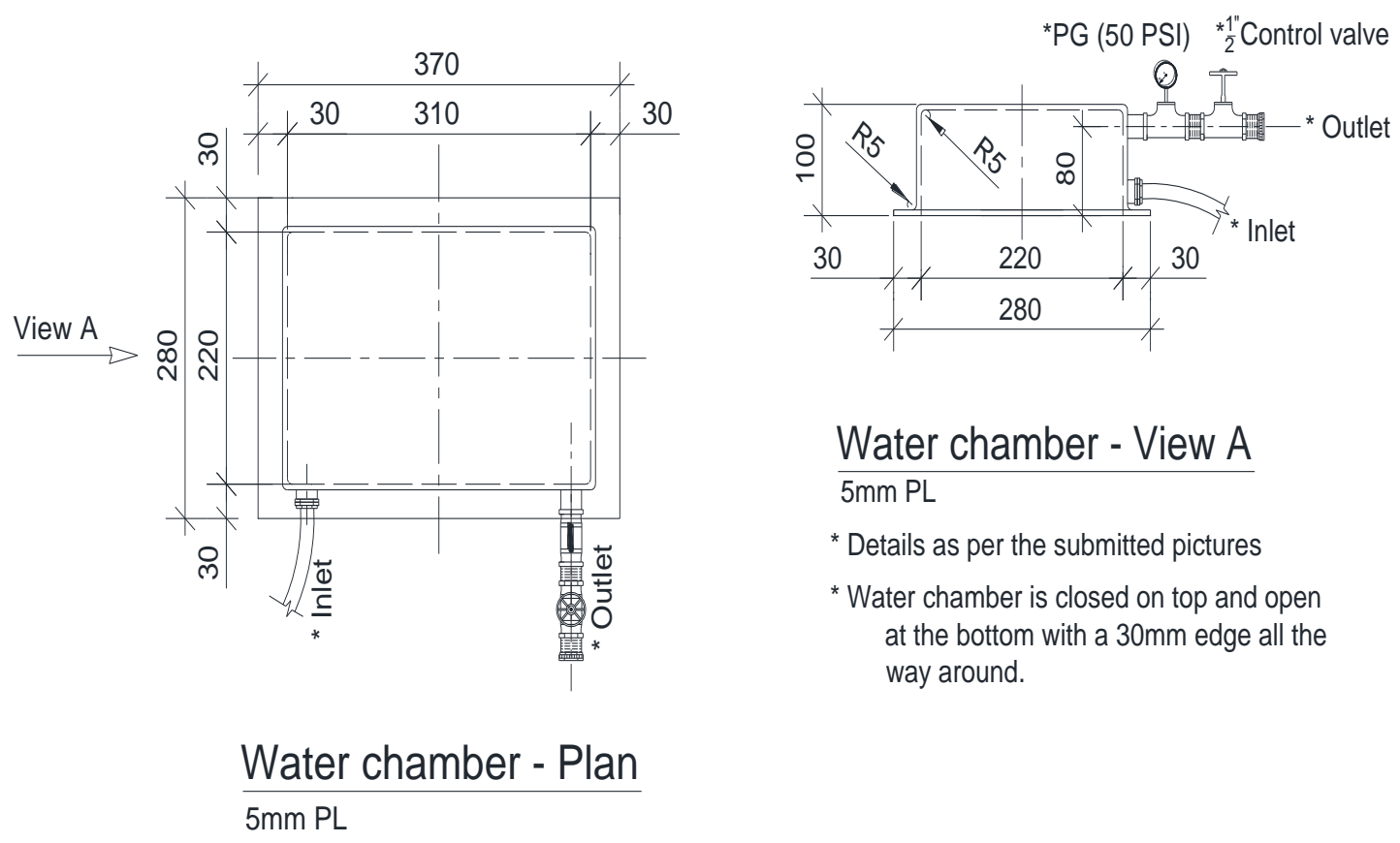

\section{Water chamber - View A \\ $5 \mathrm{~mm} \mathrm{PL}$}

${ }^{*}$ Details as per the submitted pictures

* Water chamber is closed on top and open at the bottom with a $30 \mathrm{~mm}$ edge all the way around.

Figure 3.3: Details of the water pressure chamber 


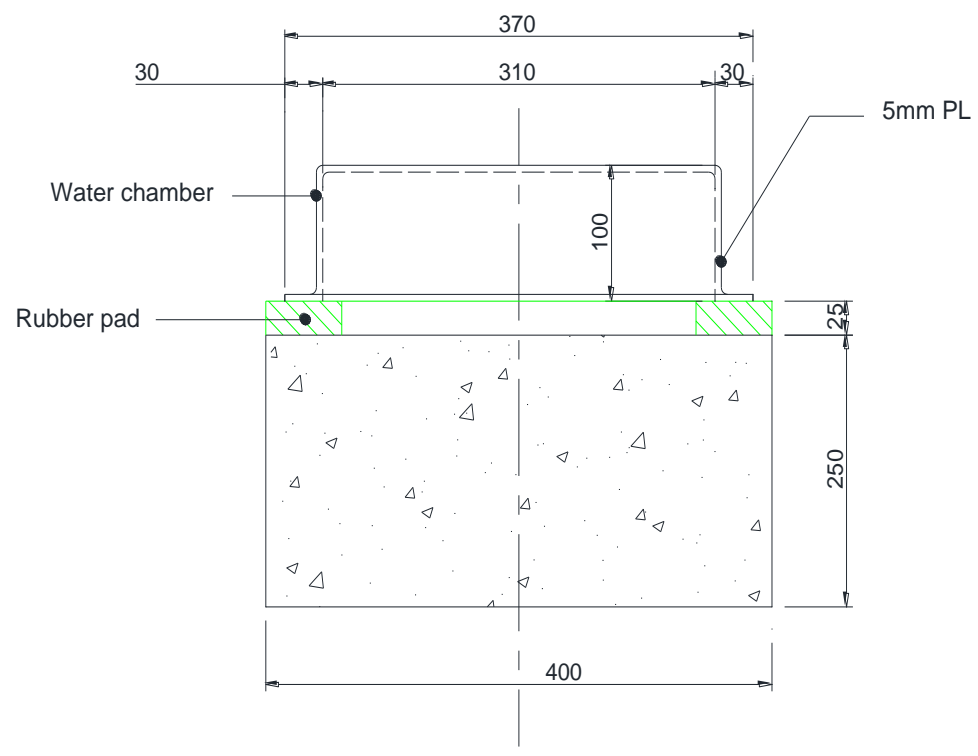

Figure 3.4: Water chamber mounted on the specimen

\section{3-2 Materials}

This section describes the mix design of the NC and ECC, as well as material properties of reinforcement, high-strength epoxy, FRP laminates, and concrete materials.

\section{3-2-1 Concrete materials and properties}

Mainly three types of concrete including NC, ECC slag and ECC fly ash are used and the 350-liter shear drum mixer is used. The water to cementitious materials of the ECC mix is 0.27 . ECC mix ingredients include water, general purpose (GU) cement, slag and fly ash (FA) as cementitious materials, natural grain silica sand with nominal size of 110 micrometer, Polyvinyl Alcohol (PVA) fiber, and a poly-carboxylate based high range water reducer (HRWR) as illustrated in Table 3.1. The PVA fiber used in the ECC mix design, which is shown in Figure 3.5, has a diameter of 39 microns, length of $8 \mathrm{~mm}$, tensile strength of $1620 \mathrm{MPa}$, modulus of elasticity of $42.8 \mathrm{GPa}$, as well as a melting point of $225^{\circ} \mathrm{C}$. For ECC mixing, GU cement, fly ash or slag, and silica sand were 
introduced to the shear mixer and mixed for one minute. Then $50 \%$ of the HRWR and $75 \%$ of water were mixed and added to the mixer gradually and mixed with other materials for 2 to 3 minutes. After that, the remaining of the water and HRWR were added to the mix with the same procedure and mixed for an additional 2 minutes to achieve the desirable flow-ability and consistency. Finally, the PVA fibers was added to the shear mixer and mixed for 3 minutes until full dispersion of fibers with mortar mixture. The mixing procedure of ECC is shown in Figure 3.5 .

Table 3.1: Mix design of ECC and NC

\begin{tabular}{|c|c|c|c|c|c|c|c|}
\hline \multirow{2}{*}{ Mixture } & \multicolumn{5}{|c|}{ Ingredients per 1 part of cement } & & \multirow{2}{*}{ W/B } \\
\cline { 2 - 8 } & Cement & Fly Ash (FA) & Slag & Silica sand & $\begin{array}{c}\text { PVA } \\
\mathrm{kg} / \mathrm{m}^{3}\end{array}$ & $\begin{array}{c}\text { HRWR } \\
\mathrm{kg} / \mathrm{m}^{3}\end{array}$ & W. \\
\hline ECC- Fly ash & 1 & 1.2 & - & 0.8 & 26 & 5.7 & 0.27 \\
\hline ECC- Slag & 1 & - & 1.2 & 0.8 & 26 & 7.4 & 0.27 \\
\hline Mixture & Cement & Sand & Gravel & - & - & $\begin{array}{c}\text { HRWR } \\
\mathrm{kg} / \mathrm{m}^{3}\end{array}$ & W/B \\
\hline NC & 1 & 3.13 & 3.45 & - & - & 0.75 & 0.55 \\
\hline
\end{tabular}

Normal concrete mix design has a water to cement ratio of 0.55 . It consists of general use (GU) cement, river sand, gravel, water, and a poly-carboxylate based high range water reducer (HRWR) as presented in Table 3.1. For NC mixing, coarse and fine aggregates were introduced to the shear mixer and mixed for 4 to 5 minutes. After that, GU cement was added to the mixer and mixed for 4 minutes. Then half of the water along with half of the HRWR were added to the mix gradually and mixed for 2 to 3 minutes. Lastly, the remaining of the water and HRWR were introduced to the mix with the same procedure to develop the desirable flow-ability of the mix. 


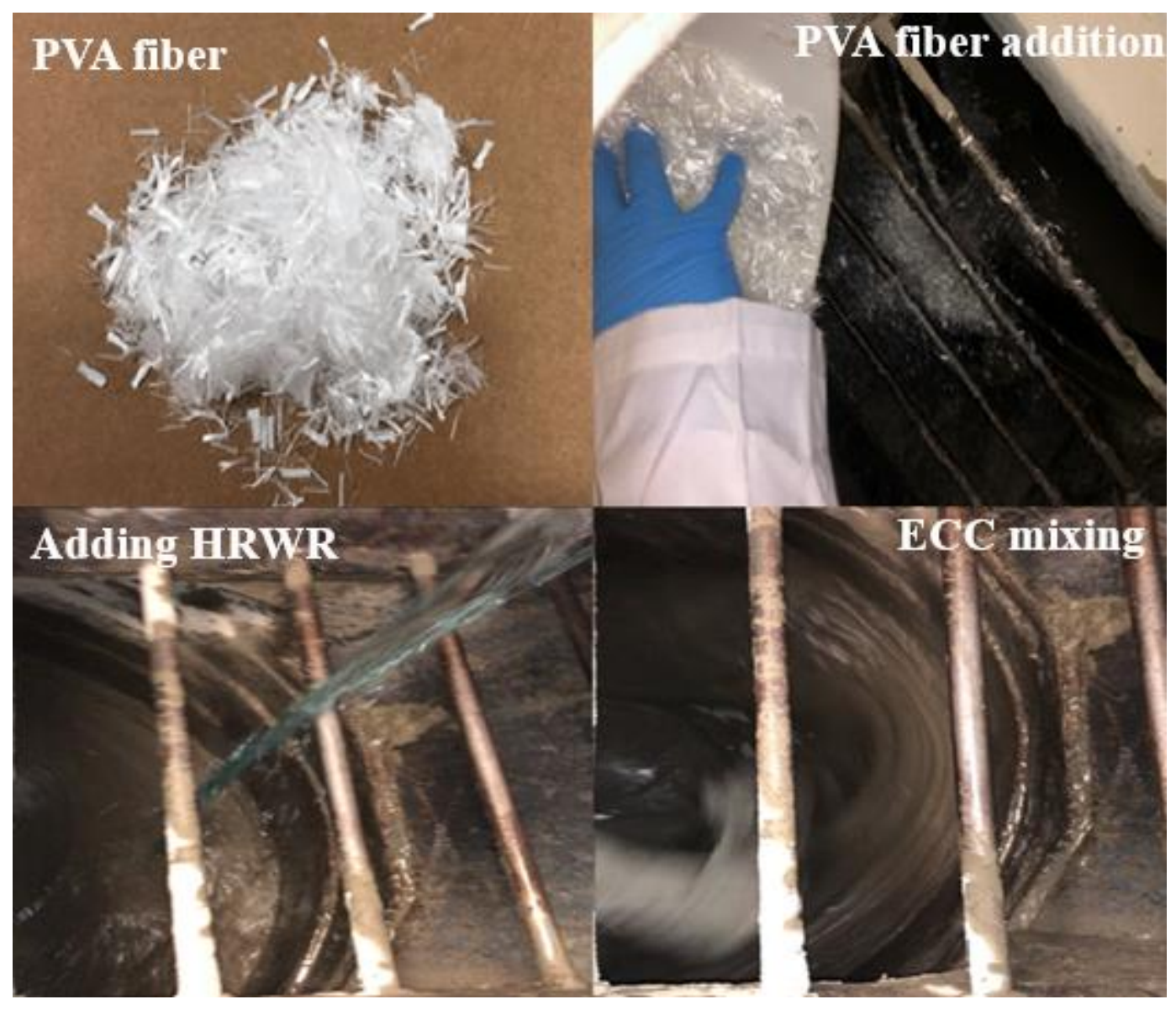

Figure 3.5: ECC mixing

\section{3-2-2 Steel reinforcement properties}

A single $35 \mathrm{~mm}$ rebar $(\rho=1 \%)$ was used as the longitudinal reinforcement for each specimen and it was placed at the center of the RC panel to prevent any eccentricity during loading. The rebar used in this experimental program was Grade 400 steel which was conforming to the Canadian Standard Association (CSA). The properties of tested rebar are shown in Figure 3.6 and Table 3.2.

Table 3.2: Properties of steel reinforcement

\begin{tabular}{|c|c|c|c|}
\hline $\begin{array}{c}\text { Rebar diameter } \\
(\mathrm{mm})\end{array}$ & $\begin{array}{c}\text { Yield stress } \\
(\mathrm{MPa})\end{array}$ & $\begin{array}{c}\text { Yield Strain } \\
(\text { Micro-strain })\end{array}$ & $\begin{array}{c}\text { Modulus of elasticity } \\
(\mathrm{MPa})\end{array}$ \\
\hline 35 & 400 & 2000 & 200000 \\
\hline
\end{tabular}




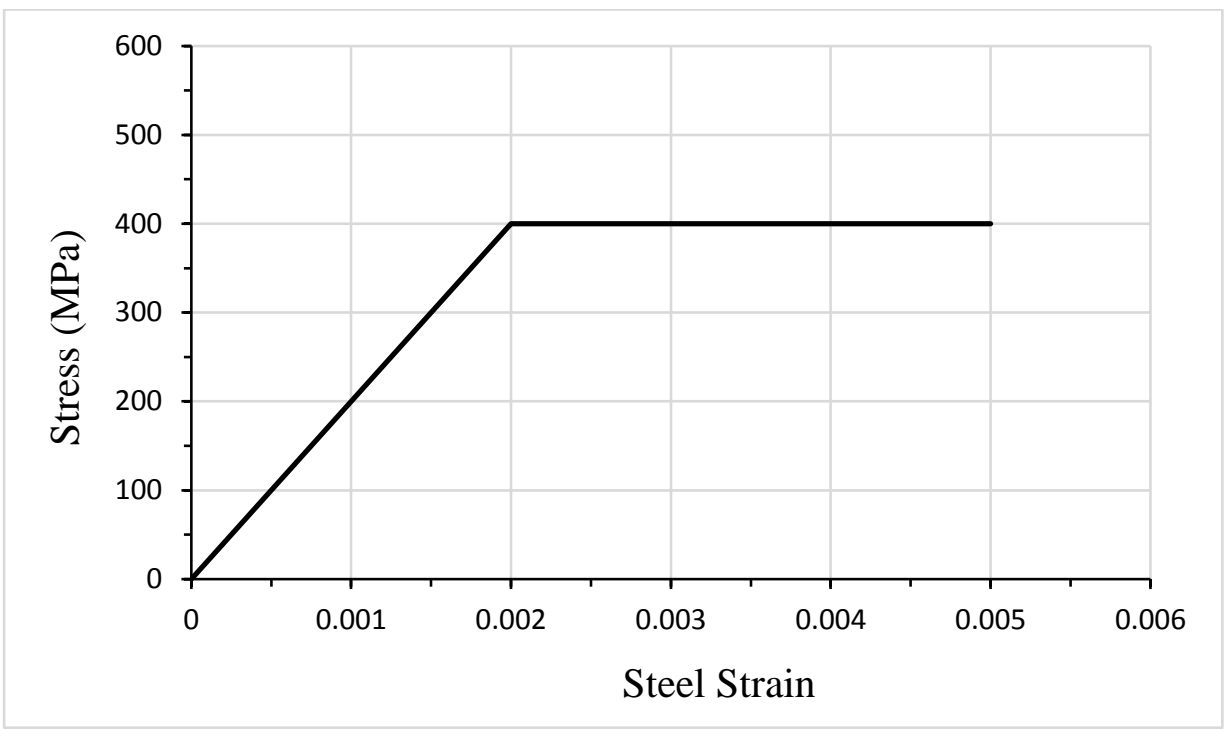

Figure 3.6: Rebar stress-strain curve

\section{3-2-3 High strength epoxy properties}

A high-strength low-viscosity epoxy was used as the repair material in this experimental program. Epoxy is a two-part product consisting of the resin and hardener that should be mixed before injection with 2:1 ratio (by volume), respectively. A high-pressure injection method was used to ensure that the epoxy penetrated through the depth of the section filling the crack. The epoxy has a low viscosity of $375 \mathrm{cps}$, as can be seen in Figure 3-7, which allows penetration and sealing of fine, hairline cracks. Epoxy starts to harden after 3-3.5 hours at room temperature $\left(23^{\circ} \mathrm{C}\right)$ and it can achieve a high early strength of up to 5 times stronger than concrete in a short curing time. It is a moisture tolerant adhesive that can be used for "can't dry" cracks and surfaces. However, it cannot be used for injection of cracks under active hydrostatic pressure. Also, it is not suitable to be injected into cracks greater than $6 \mathrm{~mm}$ in width. This type of epoxy conforms to current ASTM C881 (2015) and AASHTO M 235-13 (2018) specifications. The material properties of epoxy at room temperature $\left(23^{\circ} \mathrm{C}\right)$ are shown in Table 3.3. 


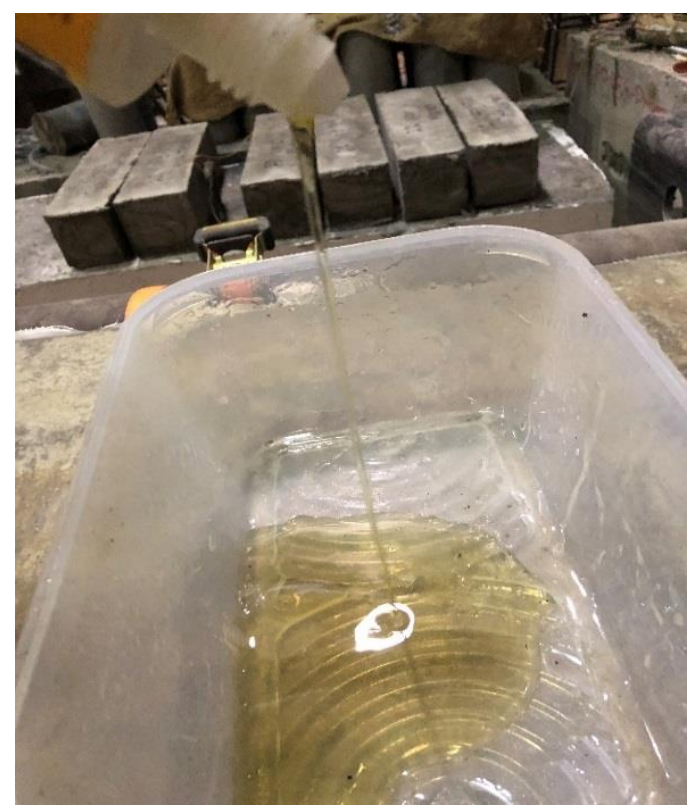

Figure 3.7: Low viscosity of the high-strength epoxy

Table 3.3: Material properties of epoxy at room temperature $\left(23^{\circ} \mathrm{C}\right)$

\begin{tabular}{|c|c|c|c|c|c|c|c|}
\cline { 2 - 8 } \multicolumn{1}{c|}{} & $\begin{array}{c}\text { Compressive } \\
\text { strength } \\
(\mathrm{MPa})\end{array}$ & $\begin{array}{c}\text { Tensile } \\
\text { strength } \\
(\mathrm{MPa})\end{array}$ & $\begin{array}{c}\text { Elongation } \\
\text { at break }(\%)\end{array}$ & $\begin{array}{c}\text { Flexural } \\
\text { strength } \\
(\mathrm{MPa})\end{array}$ & $\begin{array}{c}\text { Bond } \\
\text { strength } \\
(\mathrm{MPa})\end{array}$ & $\begin{array}{c}\text { Shear } \\
\text { strength } \\
(\mathrm{MPa})\end{array}$ & $\begin{array}{c}\text { Compressive } \\
\text { modulus } \\
(\mathrm{MPa})\end{array}$ \\
\hline 4-hours & - & - & - & - & - & - & - \\
\hline 8-hours & 1.2 & - & - & - & - & - & - \\
\hline 16-hours & 31.1 & - & - & - & - & - & - \\
\hline 1-day & 41.4 & - & - & - & - & - & - \\
\hline 2-day & - & - & - & - & 9 & - & - \\
\hline 3-day & 62.1 & - & - & - & - & - & - \\
\hline 7-day & 75.9 & 48.3 & 6.9 & - & - & - & 2000 \\
\hline 14-day & 82.8 & - & - & 75.9 & 9.3 & 33.1 & - \\
\hline 28-day & 89.7 & - & - & - & - & - & - \\
\hline
\end{tabular}

\section{3-2-4 Glass fiber reinforced polymer (GFRP) laminates properties}

Fiber reinforced polymer (FRP) is a composite material made of a polymer matrix reinforced with fibers. The common types of fiber used in industry are Glass, Carbon, Aramid, and Basalt. Glass fiber reinforced polymer (GFRP) laminate was used for this experimental program to repair the 
RC panels, which is shown in Figure 3.8. A unidirectional E-glass type of fiber fabric was used in conjunction with epoxy laminating resin to provide composite strengthening system. GFRP laminate is flexible and can be wrapped around complex shapes. Being non-corrosive, acid resistant, and proved good resistance to impact are among other advantages of the GFRP laminates. It is also light-weight and has minimum impact on the aesthetic of the structure. E-glass fiber properties are presented in Table 3.4.

The epoxy resin system, used in conjunction with the glass fiber fabric, is composed of two parts including the resin and hardener. A slow hardener was chosen for this experimental program to provide sufficient time for mixing the resin and hardener and applying on the concrete surface properly. Moreover, the hardener was chosen to be low-viscosity so that the epoxy resin system can quickly wet out reinforcing glass fiber fabrics when laminating. The epoxy resin system provides excellent moisture resistance which makes it an appropriate choice for repairing projects exposed to moisture. The properties of the resin, hardener, and epoxy resin system are presented in Table 3-5. Through-cure and time to sand varies based on the type of hardener (Fast, slow, very slow), due to their various gel times. For slow hardener type chosen for this project, the time to sand is about 6-7 hours. It will cure hard overnight and achieve the full properties after 7 days of standard curing at 21 to $24^{\circ} \mathrm{C}$. Properties of the cured laminate after standard curing ( 7 days at 21 to $24^{\circ} \mathrm{C}$ ) are described in Table 3.6. 


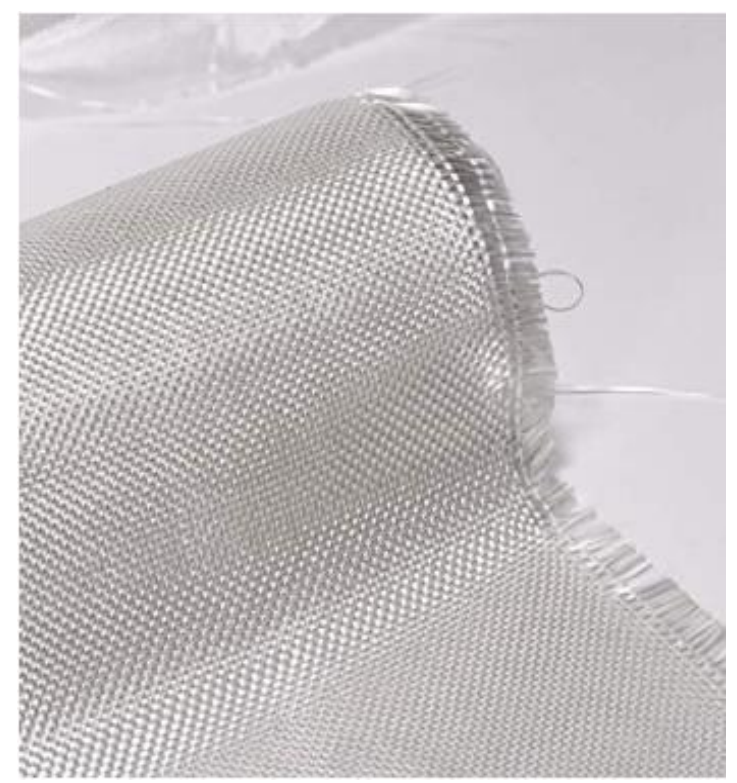

Figure 3.8: Glass fiber fabric

Table 3.4: E-glass fiber fabric properties

\begin{tabular}{|c|c|c|c|c|c|}
\hline Primary fiber direction & Tensile strength & $\begin{array}{c}\text { Tensile } \\
\text { E-modulus }\end{array}$ & Elongation & Density & Area weight \\
\hline 0 Unidirectional & $2.25 \mathrm{GPa}$ & $70 \mathrm{GPa}$ & $2.80 \%$ & $2540 \mathrm{~kg} / \mathrm{m}^{3}$ & $430 \mathrm{gr} / \mathrm{m}^{2}$ \\
\hline
\end{tabular}

Table 3-5: Properties of the resin, hardener, and epoxy resin system

\begin{tabular}{|c|c|c|c|}
\hline & Resin & Slow hardener & Epoxy resin system \\
\hline Color & Light Amber & Amber & Light Amber \\
\hline Viscosity (cps) & 850 & 200 & 575 \\
\hline Specific gravity & 1.14 & 0.99 & 1.11 \\
\hline Mix ratio (by volume) & - & 5 to 1 & - \\
\hline Pot life at 77 F & - & 23 minutes & 23 minutes \\
\hline
\end{tabular}

Table 3.6: Cured laminate properties after standard curing (7 days at 21 to $24^{\circ} \mathrm{C}$ )

\begin{tabular}{|c|c|c|c|c|}
\cline { 2 - 5 } \multicolumn{1}{c|}{} & $\begin{array}{c}\text { Tensile strength } \\
(\mathrm{MPa})\end{array}$ & $\begin{array}{c}\text { Tensile modulus } \\
(\mathrm{MPa})\end{array}$ & $\begin{array}{c}\text { Tensile } \\
\text { elongation }(\%)\end{array}$ & $\begin{array}{c}\text { Nominal laminate } \\
\text { thickness (mm) }\end{array}$ \\
\hline Average value & 611 & - & 2.24 & - \\
\hline Design value & 541 & 27400 & 1.82 & 1.016 \\
\hline
\end{tabular}




\section{3-3 Specimen fabrication, casting and curing}

The fabrication and casting of the specimens were done in two stages including NC panels and the ECC repair layer. As the NC panels, a total number of 4 specimens were cast using normal concrete (NC) in a prepared wooden form-work. Figure 3.9 illustrates the typical form-work along with the rebar located in the center of the cross section, position of the steel strain gauges, as well as the notch and two thin membrane sheets in the middle of the specimen length.

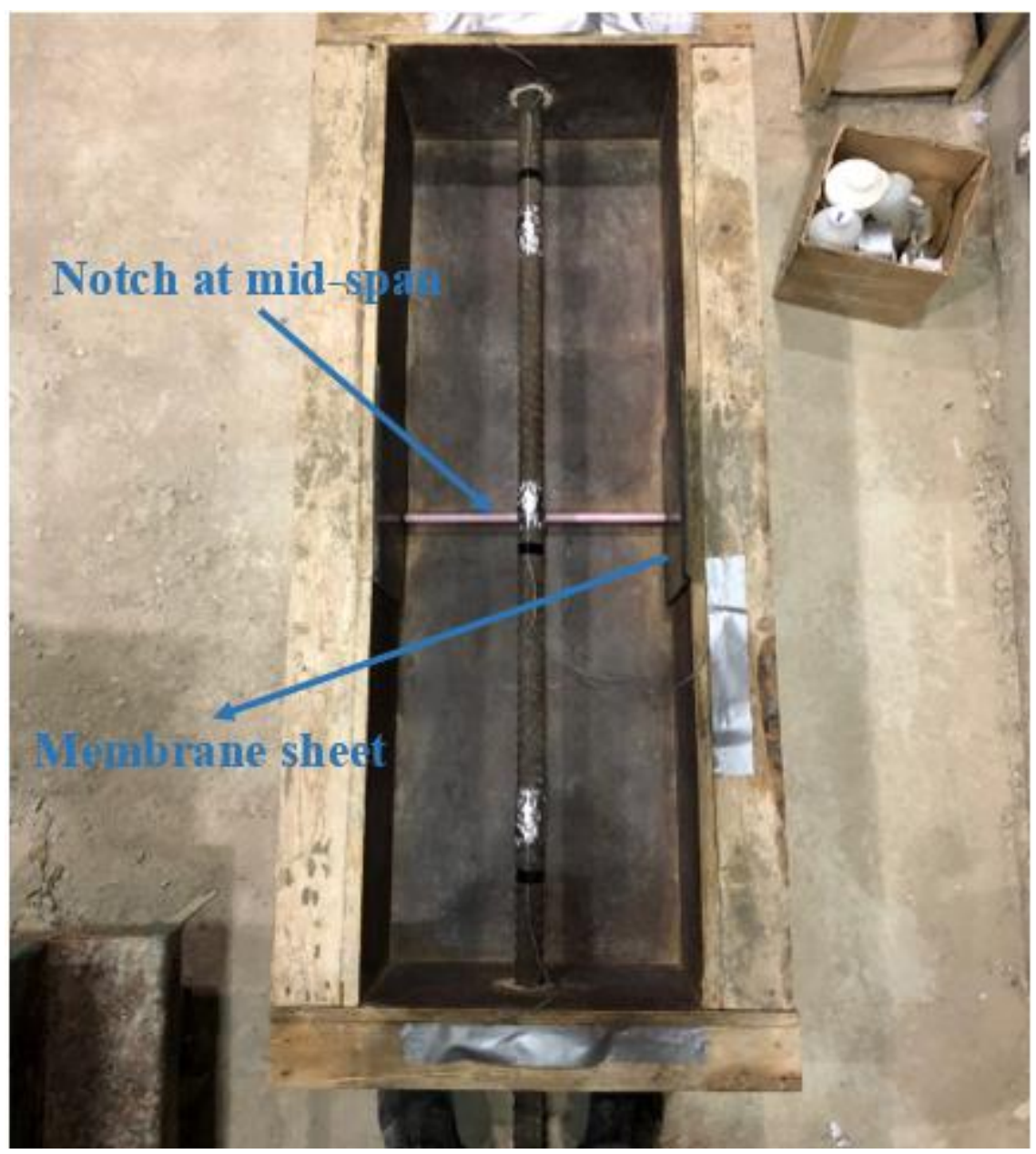

Figure 3.9: Typical RC panel form work

A total volume of 200 liter of normal concrete (NC) was used for each specimen along with the control samples. After mixing procedure, fresh concrete was directly poured to the form-work in two layers and mechanical vibration was used to ensure proper compaction of the concrete. From 
each batch, 12-cylinder samples with $200 \mathrm{~mm}$ height and $100 \mathrm{~mm}$ diameter were cast for tensile strength, 7-day compressive strength, and 28-day compressive strength tests. Cast specimen is shown in Figure 3.10.

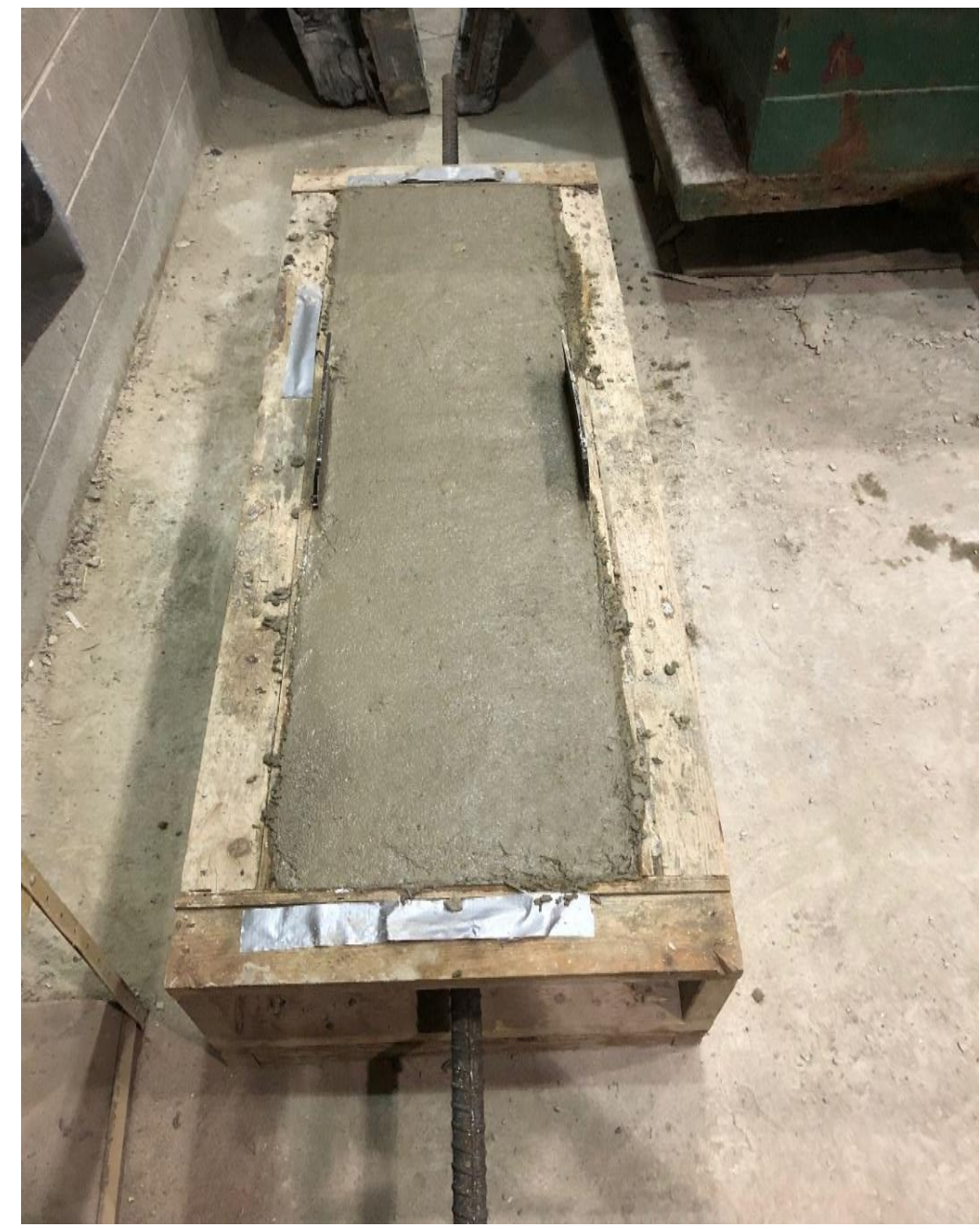

Figure 3.10: Typical reinforced normal concrete panel after casting

The specimens and poured controlled samples were covered with impermeable sheets right after the casting. The form-works for all the samples were removed 72 hours after the casting. Specimens were moist cured until the age of 7 days using wet burlaps in the relative humidity of $50 \% \pm 2.5 \%$ and temperature of $24 \pm 2{ }^{\circ} \mathrm{C}$ in the laboratory conditions, followed by air-curing until the testing age of 28-day. Moist curing of the RC panels is shown in Figure 3.11. 


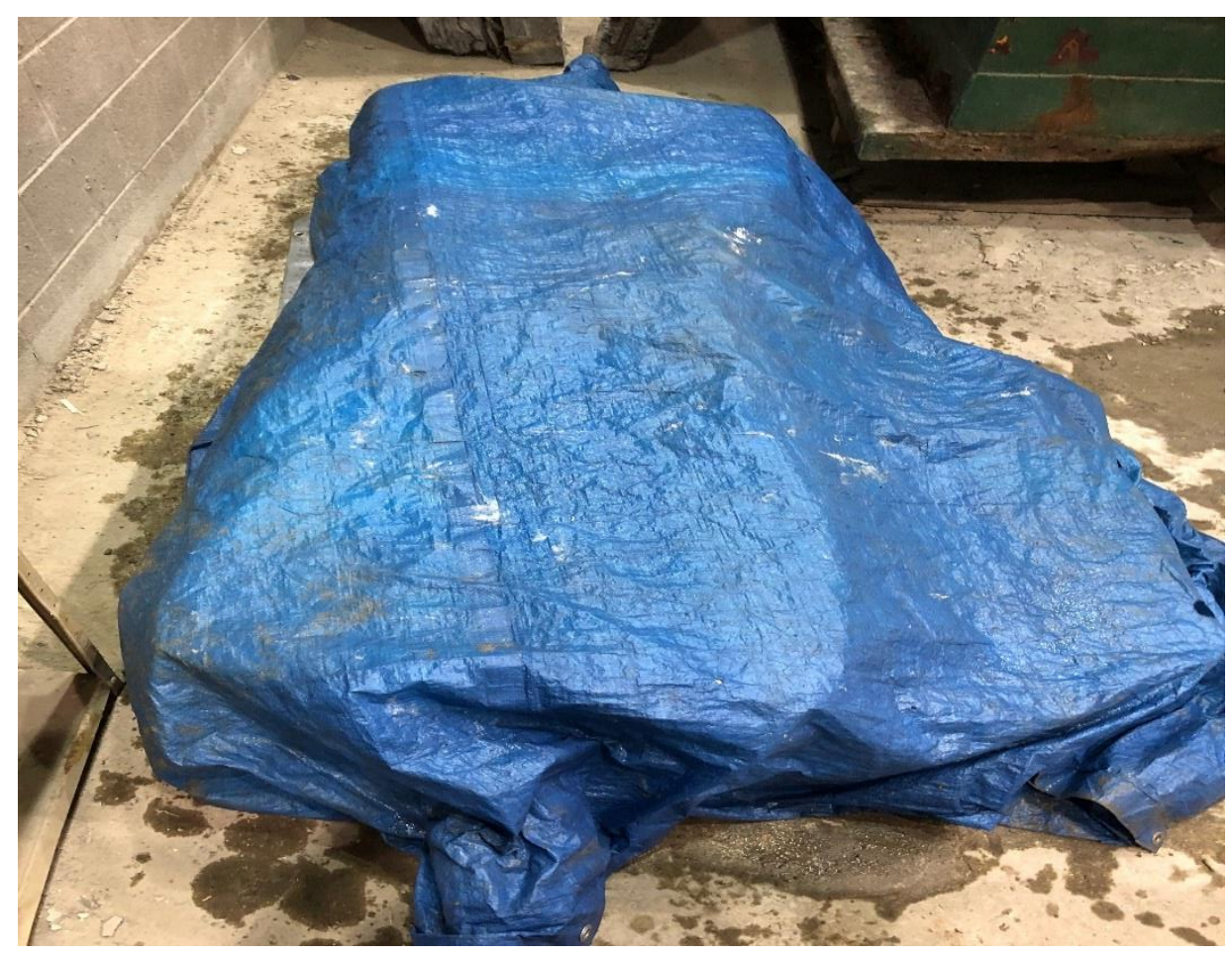

Figure 3.11: Moist curing of the RC panels after casting

After testing the NC panels under monotonic tension loading, a wooden formwork was prepared on top of the RC panel for casting the repair layer using ECC, which is shown in Figure 3.12. In order to provide proper bonding between the ECC repair layer and the main RC panel, concrete grinding was performed on the top surface of the RC panel in different locations up to the depth of half of the maximum size aggregates $(10 \mathrm{~mm})$, followed by wire brushing. Also, the RC panel surface was moistened just before casting the ECC repair layer to improve the bonding. 


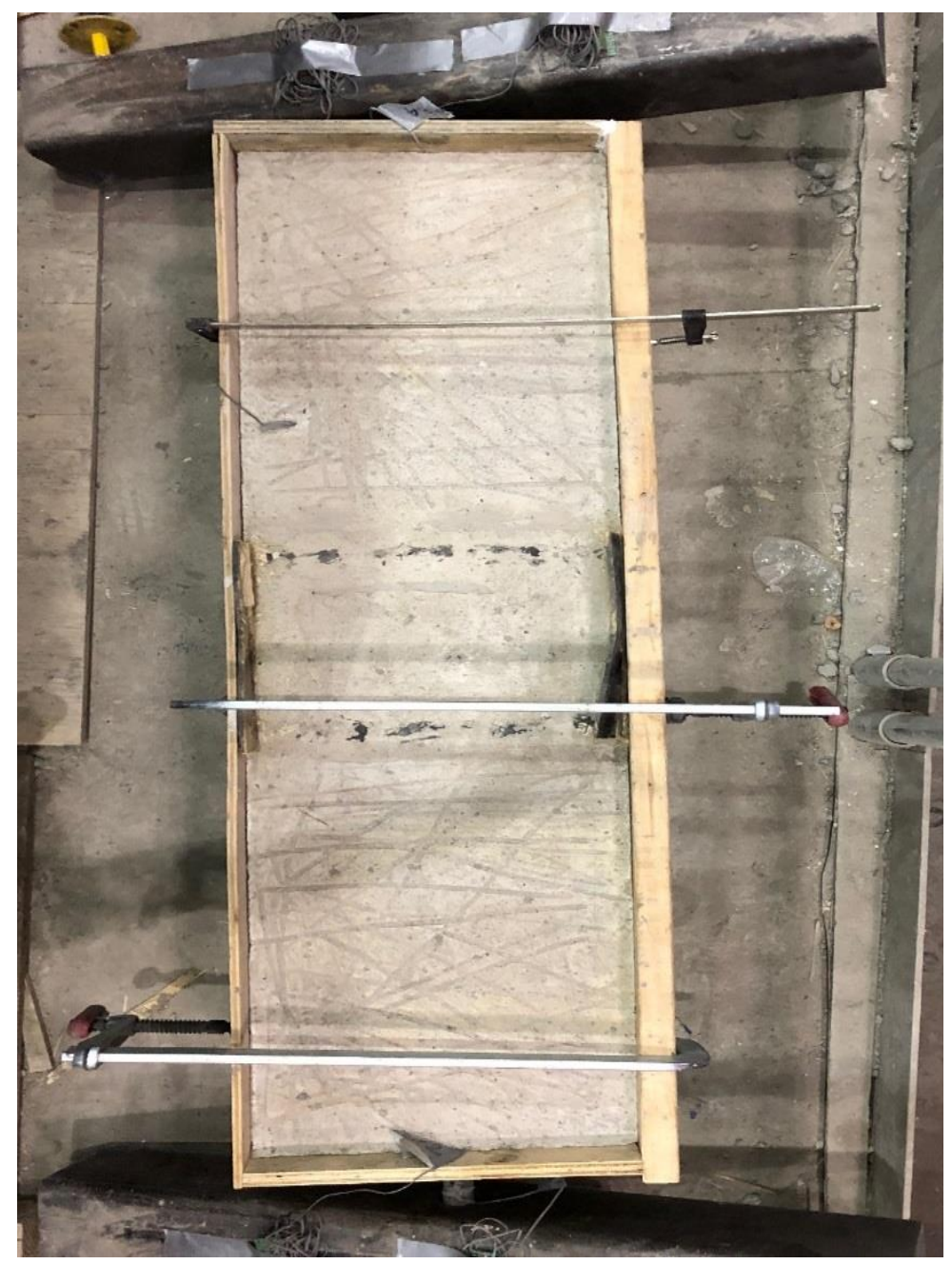

Figure 3.12: Repair layer formwork on top of the RC panel

A total volume of 100-liter ECC was used to fill the form-work along with 12 cylinders with 200 $\mathrm{mm}$ height and $100 \mathrm{~mm}$ diameter for tensile strength. 7-day compressive strength, 28-day compressive strength tests. Figure 3.13 illustrates the ECC repair layer after casting with control samples. The formwork of the ECC repair layer and control samples were removed after 72 hours of casting. The ECC samples were covered with wet burlaps and impermeable plastic sheets to provide moist curing right after the casting until the sage of 28 days. Then, specimen was air-cured until the testing age of 28-day in the laboratory conditions. 


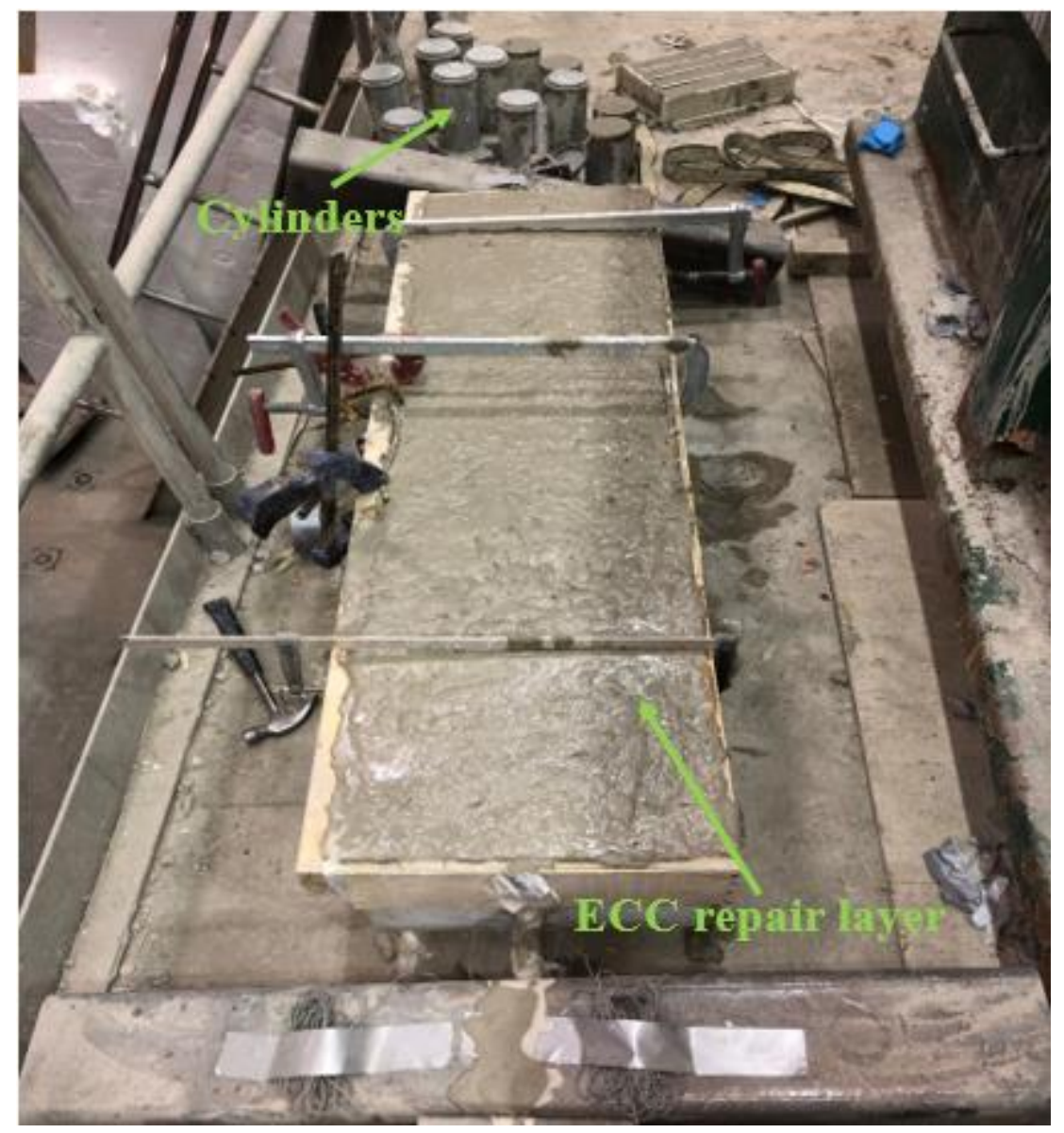

Figure 3.13: ECC repair layer after casting with control samples

The 7-day and 28-day compressive strengths, as well as 28-day tensile strength of the cylinder samples made with NC, and ECC tests were performed as per ASTM C39 (2012) and ASTM C496 (2012), respectively. The hardened ECC slag, ECC fly ash, and NC cylinder specimens after grinding are shown in Figure 3-14. Average 28-day tensile strength (based on Brazilian split cylinder test) as well as 7-day and 28-day compressive strengths of ECC and NC cylinders are presented in Table 3.7. 


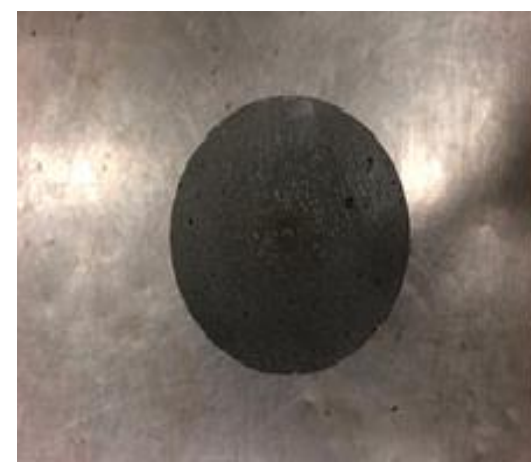

(a) ECC- Fly ash

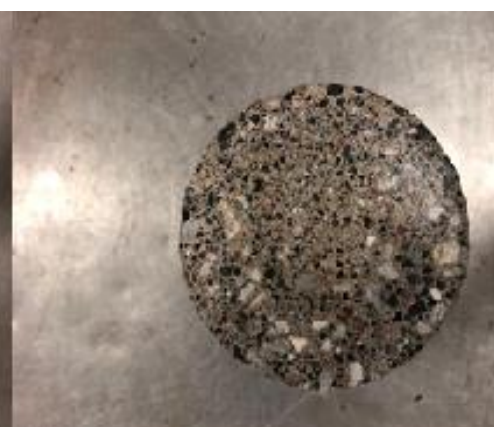

(b) NC

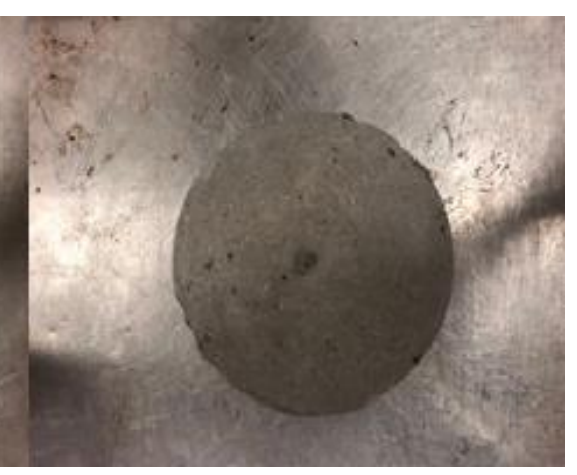

(c) ECC- Slag

Figure 3-14: Hardened cylinder specimens after grinding a) ECC-fly ash b) NC c) ECC-slag

Table 3.7: Concrete compressive and tensile strength

\begin{tabular}{|c|c|c|c|}
\hline Concrete type & $\begin{array}{c}\text { Mean 7-day compressive } \\
\text { strength (MPa) }\end{array}$ & $\begin{array}{c}\text { Mean 28-day compressive } \\
\text { strength (MPa) }\end{array}$ & $\begin{array}{c}\text { Mean 28-day indirect } \\
\text { tensile strength (MPa) }\end{array}$ \\
\hline ECC Fly ash & 56.4 & 64.4 & 6.52 \\
\hline ECC Slag & 68.2 & 75.7 & 8.26 \\
\hline NC & 31.8 & 39.4 & 2.66 \\
\hline
\end{tabular}

\section{3-4 Experimental setup, instrumentation, and testing}

A total number of 5 electrical resistance strain gauges, which were $10 \mathrm{~mm}$ long, were installed on the rebar at different locations including one in the middle of the rebar, two gauges at 1/6-span of the specimen from both ends, and two gauges outside of the specimen on both ends to ensure the proper transfer of the load from the hydraulic jack to the rebar through the solid round bars and HSS. Furthermore, two linear voltage displacement transducers (LVDTs) were connected to the sides of the RC panel in the middle section (intentionally induced crack location) to monitor the displacement with respect to the crack location that can be representative of the crack width. Crack width was also measured using crack scope with a magnification of $25 \mathrm{X}$. moreover, two load cells were placed on the sides of the specimen in contact with the hydraulic jacks to measure the applied tension load. 
Figure 3.15 and Figure 3-16 illustrate the actual side view of the RC panel along with the locations of the steel strain gauges, LVDTs, water chamber and pressure valve, hydraulic jacks and HSSs, load cells, membrane sheets, water pressure regulator and pressure gauge, and U-shape channels tightening the water chambers through $4 \times 20 \mathrm{~mm}$ steel rods to prevent any leakage in the contact area of the water chamber, and gum rubber pad on the top surface of the specimen.

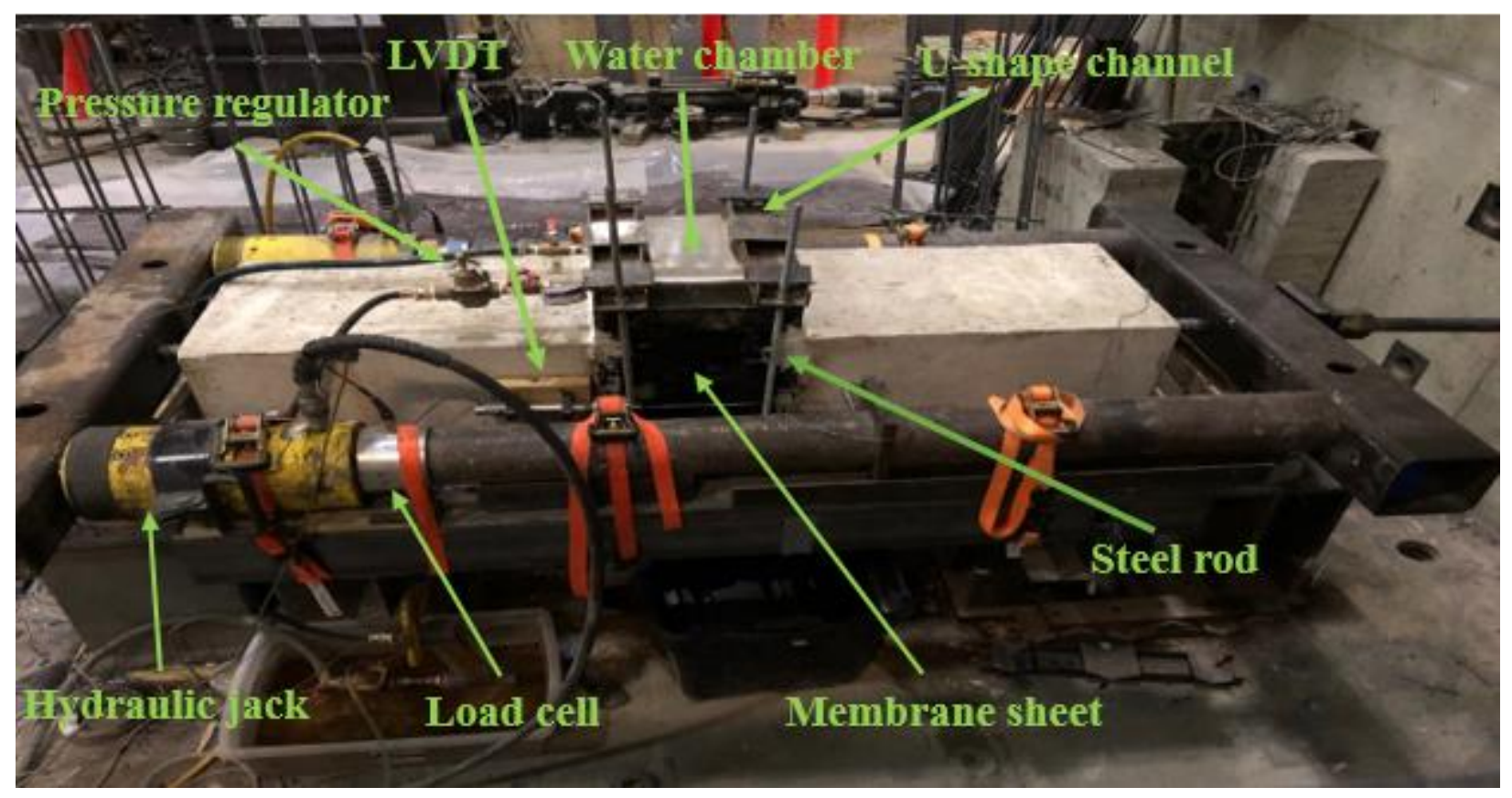

Figure 3.15: Actual side view of the specimen 


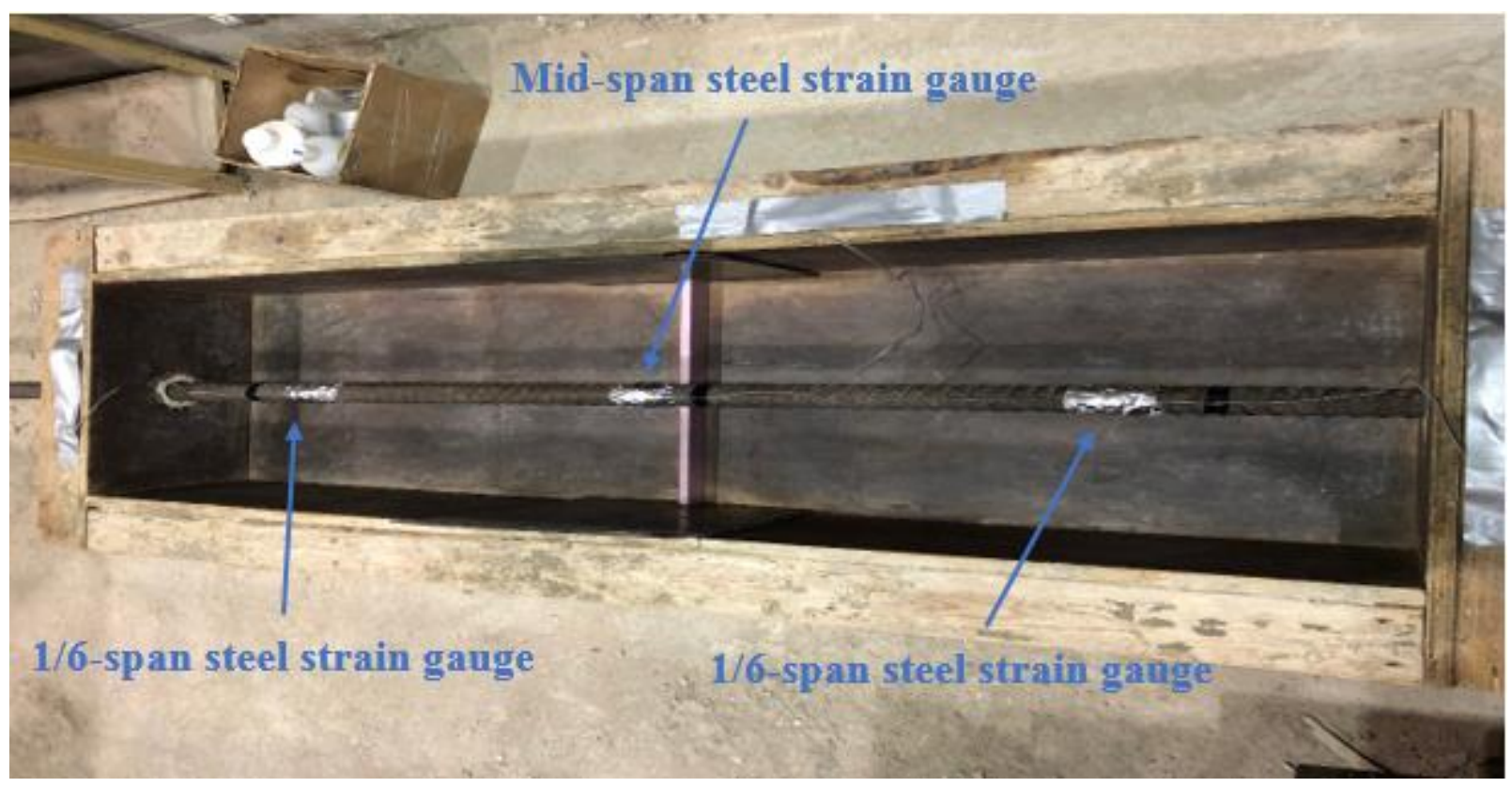

Figure 3-16: Arrangement of steel strain gauges on the rebar

\section{3-4-1 Testing procedure of RC panels}

The procedure of testing RC panels under direct tensile loading and hydrostatic water pressure is summarized in the following steps:

Step 1: Apply direct tensile loading/ major crack formation

The NC panels is carefully placed in the test setup. Steel strain gauges, LVDTs, load cells, and hydraulic jacks are in place. Figure 3-17 shows the plan view of the specimen and loading test setup. Water pressure chamber is mounted on top of the specimen and tightened using U-shape channels and four steel rods. Figure 3-18 presents the water pressure chamber mounted on top of the NC panel. Water pressure is fixed at 4 psi in the water chamber. Direct tensile loading is applied at increments of $10 \mathrm{kN}$ to the specimen. Load is increased until formation of the major crack at the pre-determined location (mid-span), as shown in Figure 3-19, along with leakage initiation. Load, strain, crack width, and displacement data are recorded at each increment. Crack width was measure using a crack scope with magnification of $25 \mathrm{X}$. 

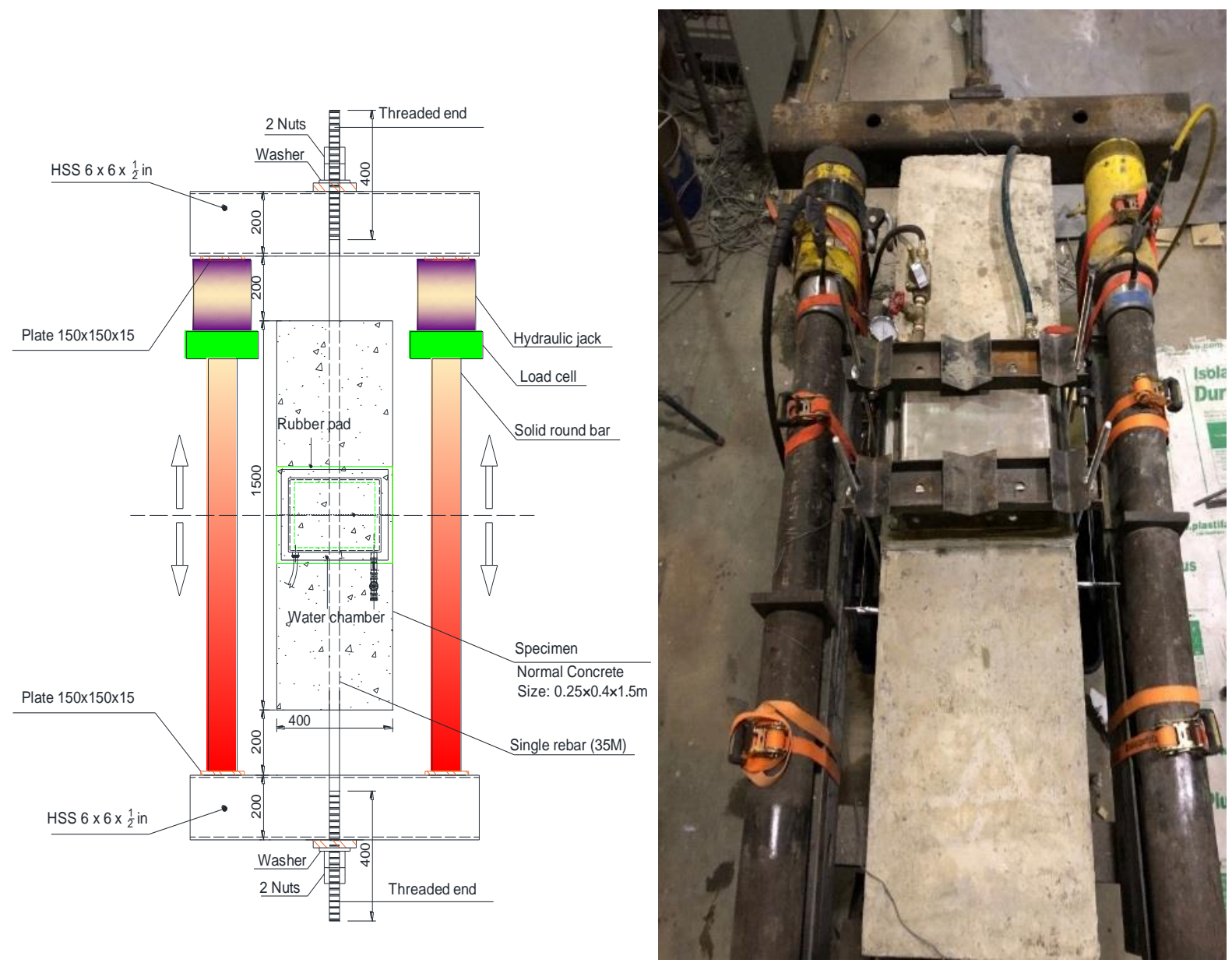

Figure 3-17: plan view of the specimen and loading test setup

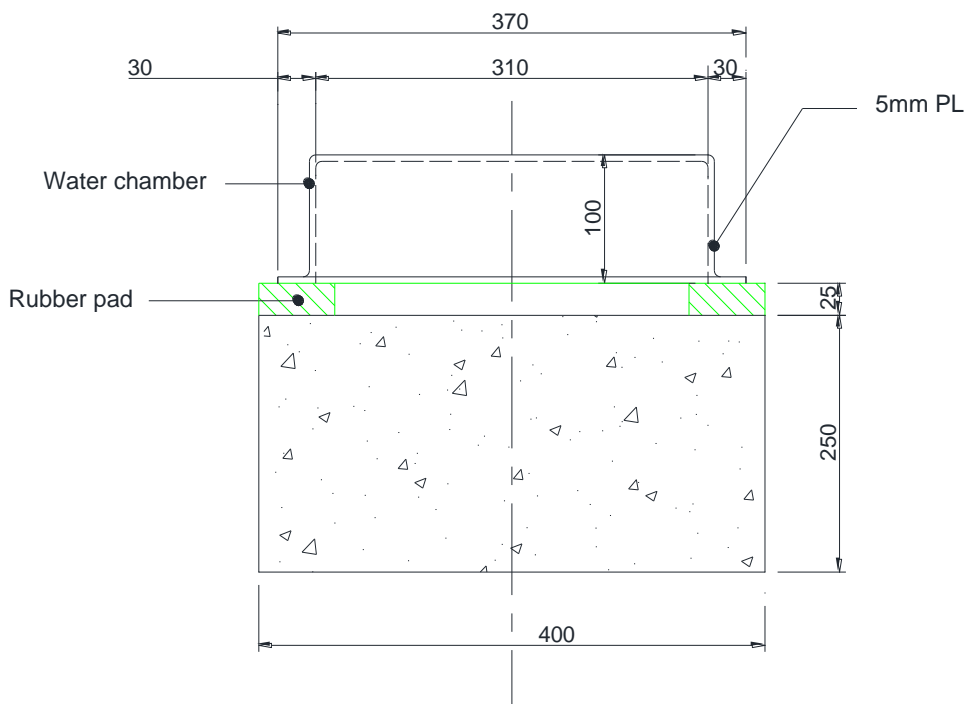

Figure 3-18: Water pressure chamber mounted on NC panel 


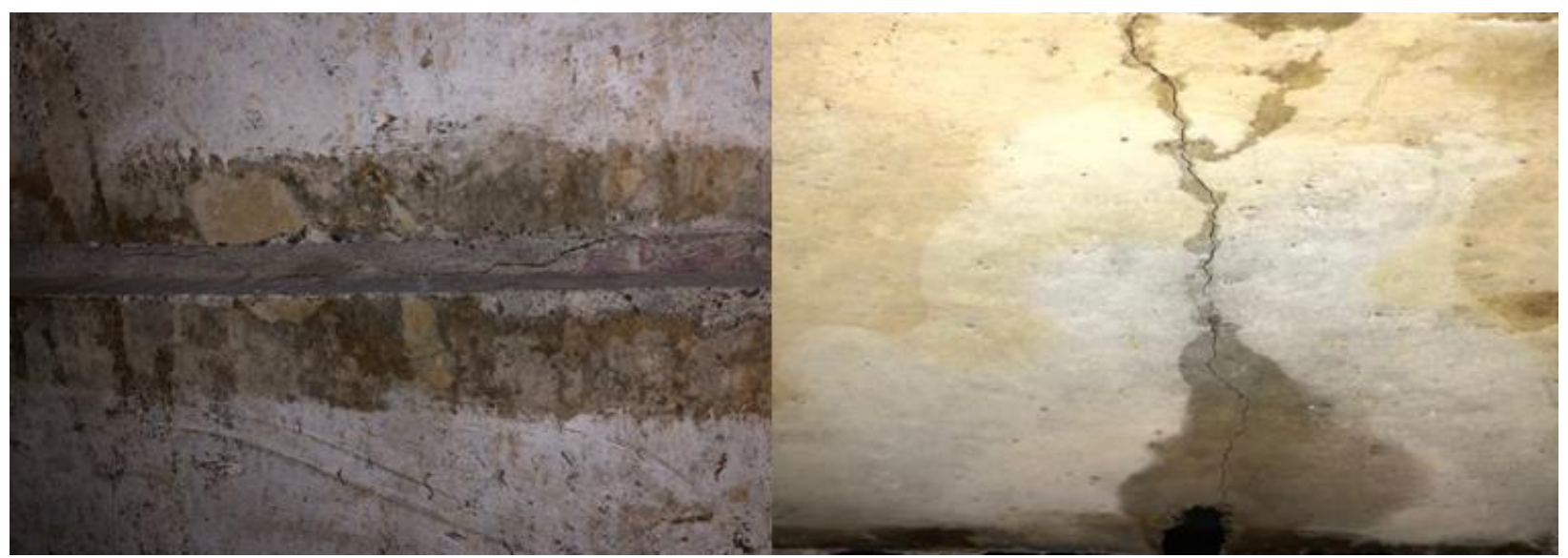

(a)

(b)

Figure 3-19: Crack formation in NC panel under direct tension a) Bottom face b) Side face

Step 2: Leakage test/ self-healing test

Load is kept increasing until the formation of a major crack at mid-span along with a constant leakage. Loading is stopped at this stage and leakage rate of the NC specimen is recorded under water pressures of 4, 8, 12, and 16 psi. Figure 3-20 shows the extreme leakage through a major crack in one of the NC specimens before repair. In order to investigate the self-healing behavior of the NC specimens under sustained tensile loading, water pressure in chamber is fixed at 7 psi and leakage rate is continuously recorded to achieve a constant leakage rate or $100 \%$ self-healing of the tension-induced cracks. Self-healing test is divided in to two parts including short-term selfhealing and long-term self-healing. In short-term investigation of the self-healing during the first 35 hours, the amount of water penetrating through the full depth of the NC panel and leaking from the bottom surface is measured continuously over one-hour time intervals (each time for 60 minutes). In long term investigation of the self-healing, during the first 35 hours leakage rate is recorded continuously each hour (each time for 60 minutes), while during the period of time between 35 hours up to the end of the test, leakage rate is measured for one-hour duration every 6 hours. 

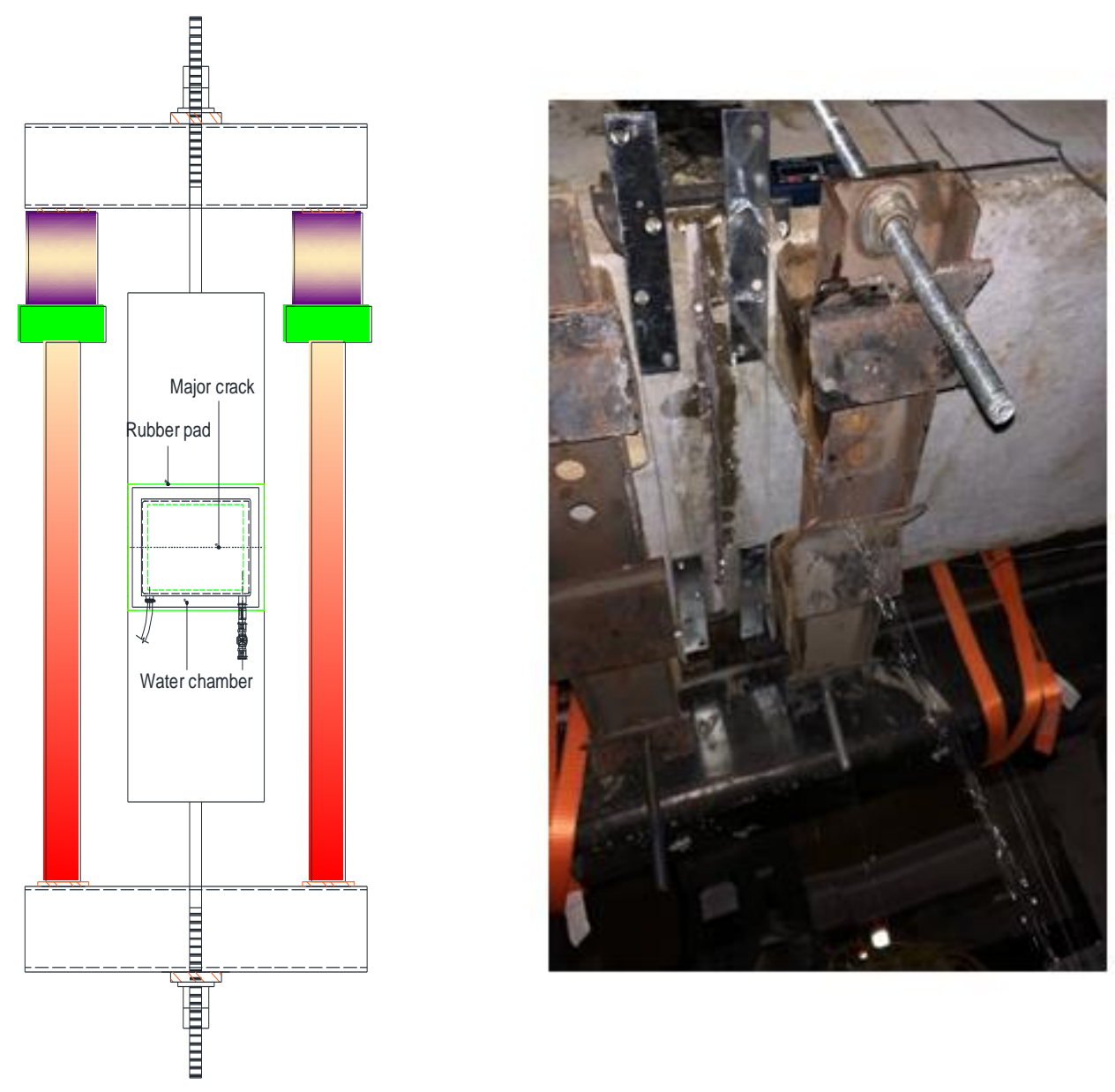

Figure 3-20: Extreme leakage through a major crack in one of the NC specimens (before repair)

Step 3: Release the tensile load/ remove water chamber/ apply repair

After measuring the leakage rate under various water pressures, tensile load is released. Also, water pressure chamber and rubber pad are removed from the top surface of the NC specimen in order to apply the repair. It is worth-mentioning that the repair materials are applied on the top surface of the NC sample, where water pressure is applied, to simulate applying the repair to the inside surface of the cracked wall of a water tank, which is exposed to water pressure. Repair materials used in this experimental program are high-strength epoxy, GFRP laminates, ECC slag, and ECC 
fly ash. Detailed procedure of applying the repair materials are discussed in section 3.4.2. Figure 3-21a illustrates the specimen after releasing the tensile load and removing the water chamber and rubber pad. Figure 3-21b shows the NC specimen with a $50 \mathrm{~mm}$ ECC repair layer. Actual NC specimens repaired with ECC and GFRP laminates are shown in Figures 3-22a and 3-22b. Moreover, Figure 3-23 illustrates the top and bottom surfaces of a NC specimen repaired with high-strength epoxy injection at mid-span crack

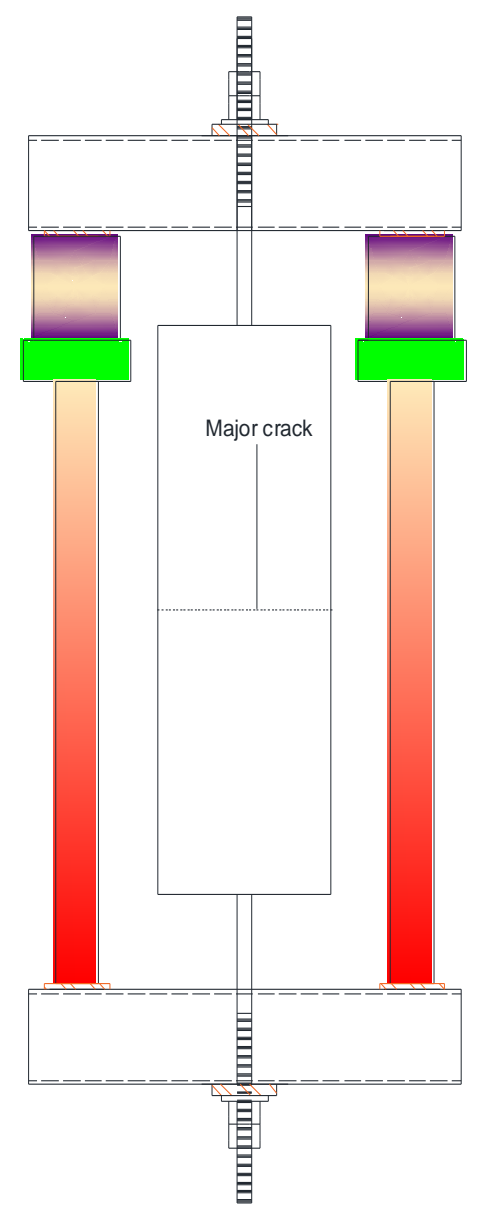

a)

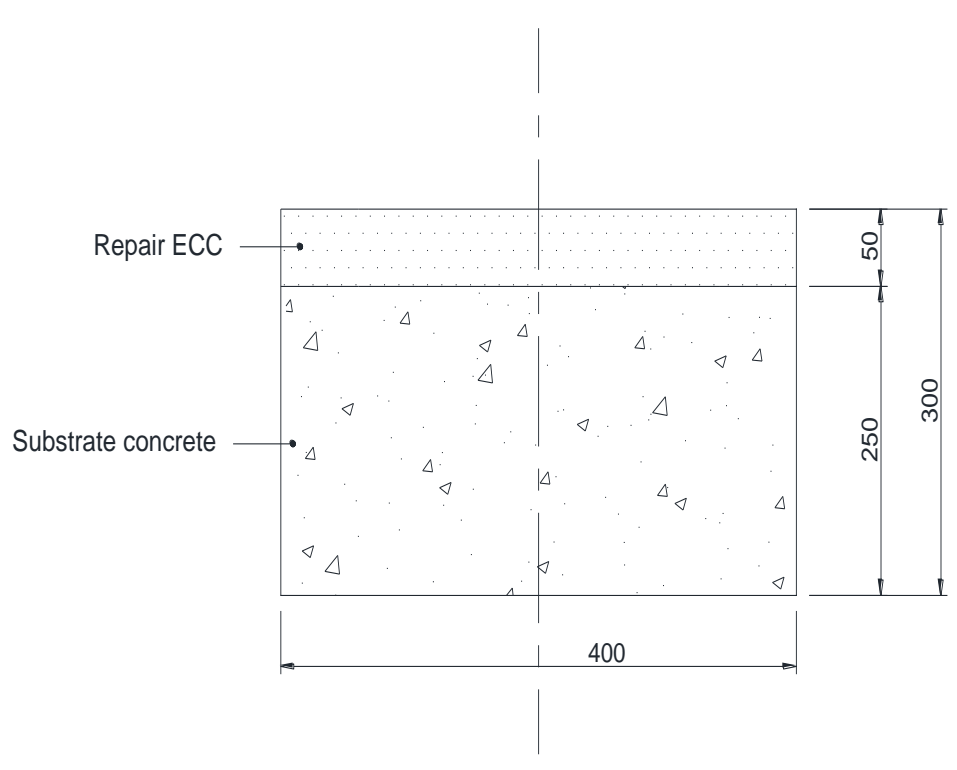

b)

Figure 3-21: Releasing the tensile load and applying the repair a) NC specimen after releasing the tensile load and removing the water chamber and rubber pad b) NC specimen after applying a $50 \mathrm{~mm}$ ECC repair layer 


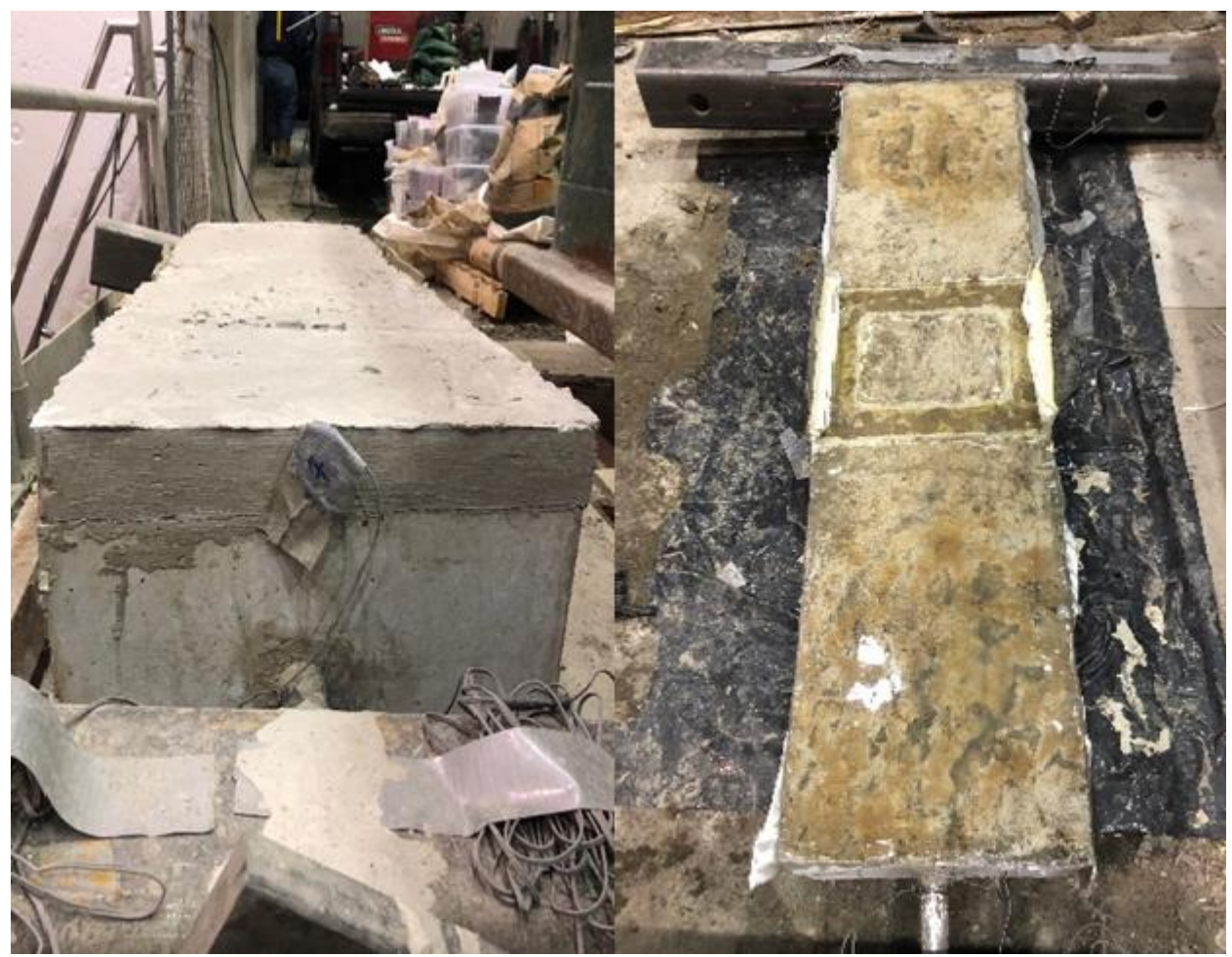

(a)

(b)

Figure 3-22: NC specimen repaired with a) ECC overlay b) GFRP laminates

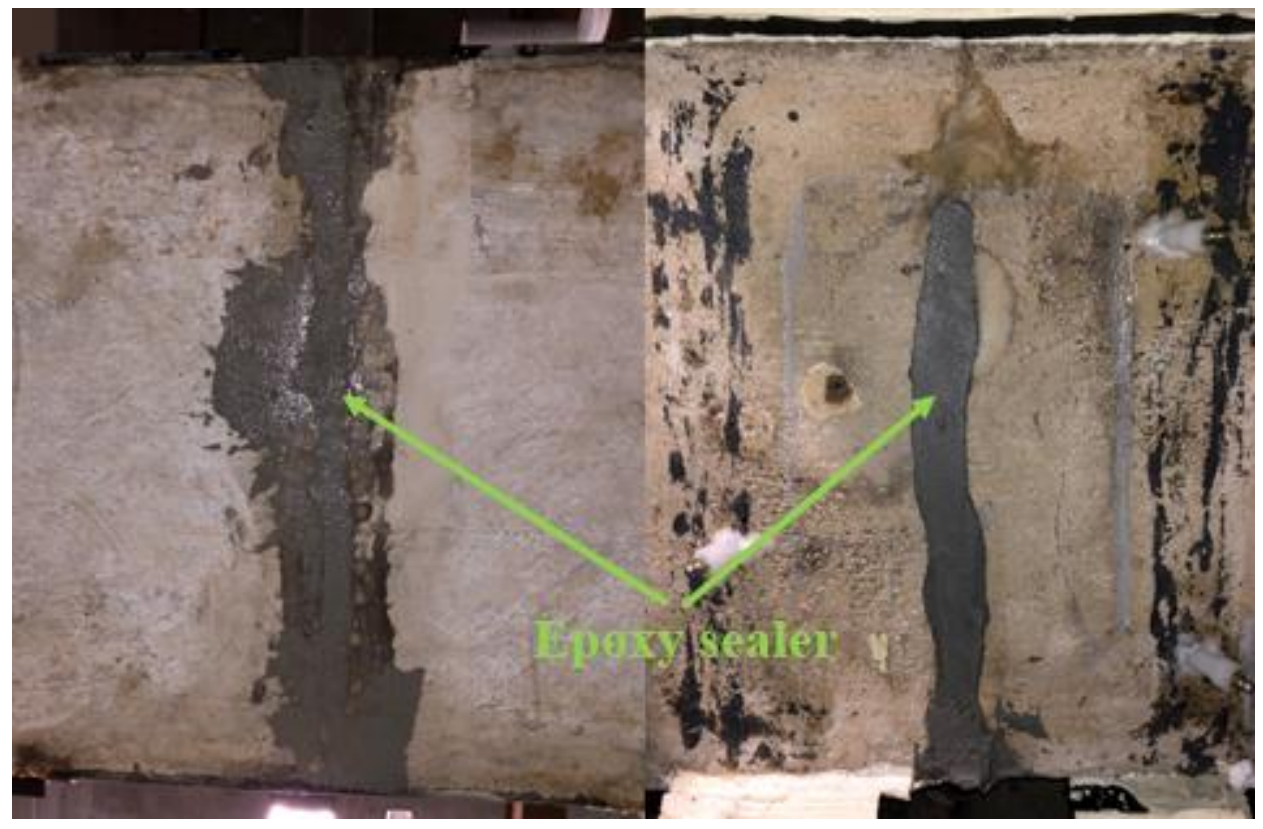

(a)

(b)

Figure 3-23: Top and bottom surfaces of a NC specimen repaired with high-strength epoxy injection at mid-span crack a) Bottom surface b) Top surface 
Step 4: Reinstall water chamber/ apply load/ leakage test

Repair materials are applied to the NC specimen and are left for curing to achieve the specified strength. Then, rubber pad and water chamber are placed again on top of the sample and tightened with steel rods and U-shape channels. Direct tensile is re-applied to the specimen at increments of $10 \mathrm{kN}$. Tensile loading is kept increasing until either failure of the repair material and leakage initiation, or reaching the yielding strain in the rebar. Leakage rate of the repaired NC specimen is measured under various water pressures of 4, 8, 12, and 16 psi. In case of reaching the yielding strain of the rebar without any failure of the repair material, test is stopped due to safety reasons. Figure 3.24 shows a repaired NC specimen in the re-load test setup. NC specimens repaired with $50 \mathrm{~mm}$ layer of ECC slag, $50 \mathrm{~mm}$ layer of ECC fly ash, 2 layers of GFRP laminates, and highstrength epoxy injection in the re-load test setup are illustrated in Figures 3-25 to Figure 3-27. 

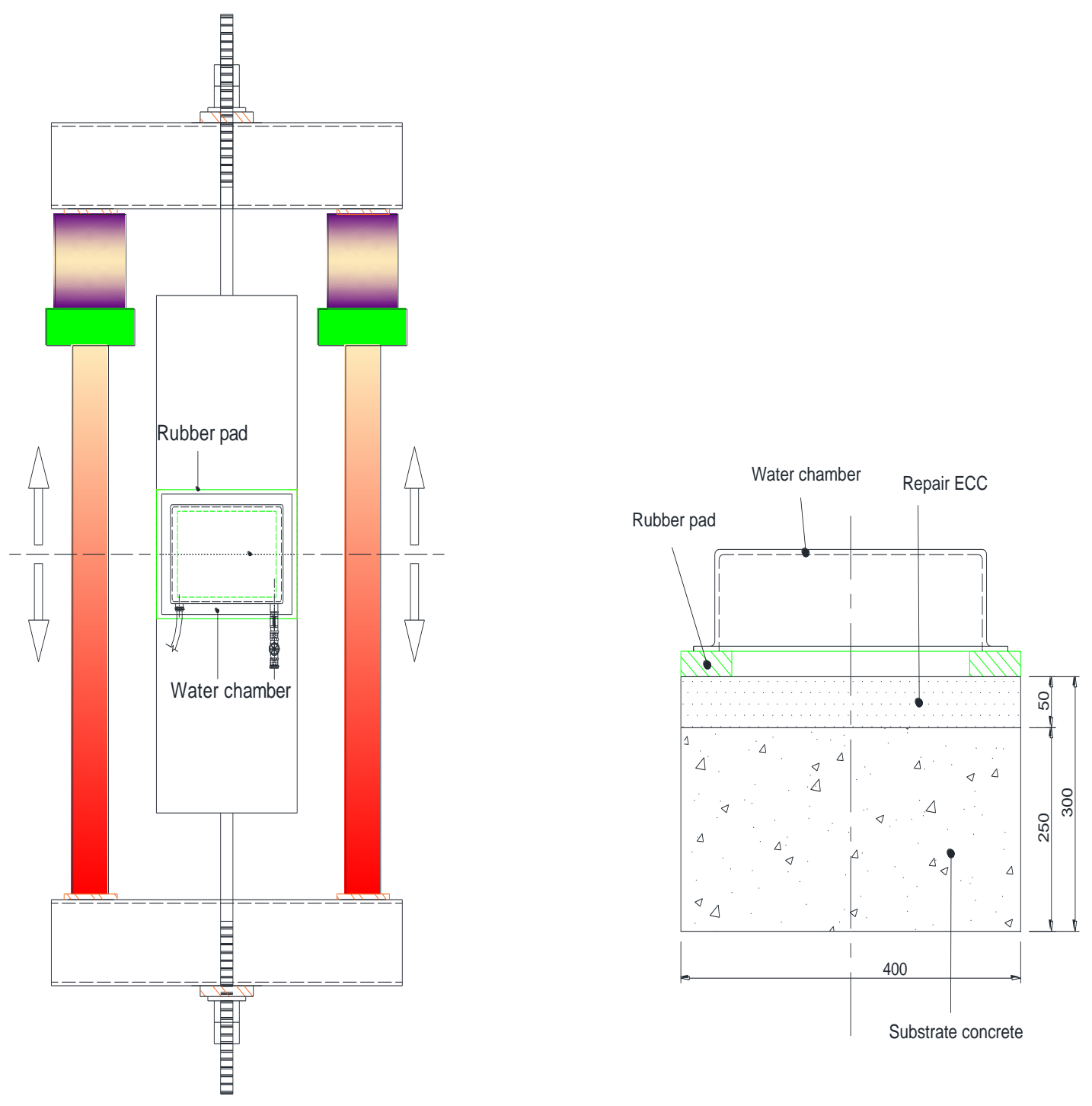

Figure 3-24: Repaired NC specimen in the re-load test setup 


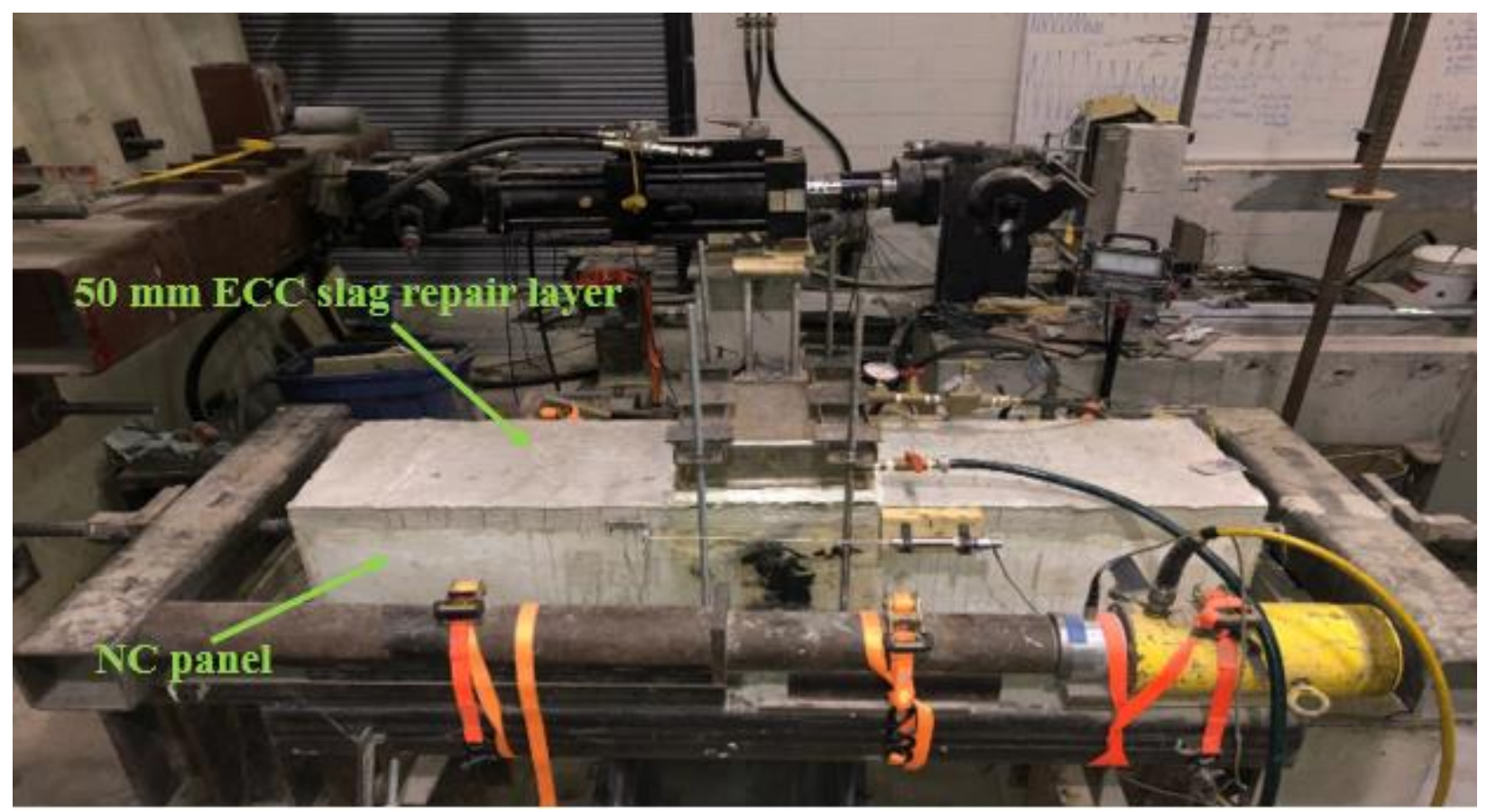

Figure 3-25: NC specimen repaired with a $50 \mathrm{~mm}$ layer of ECC slag

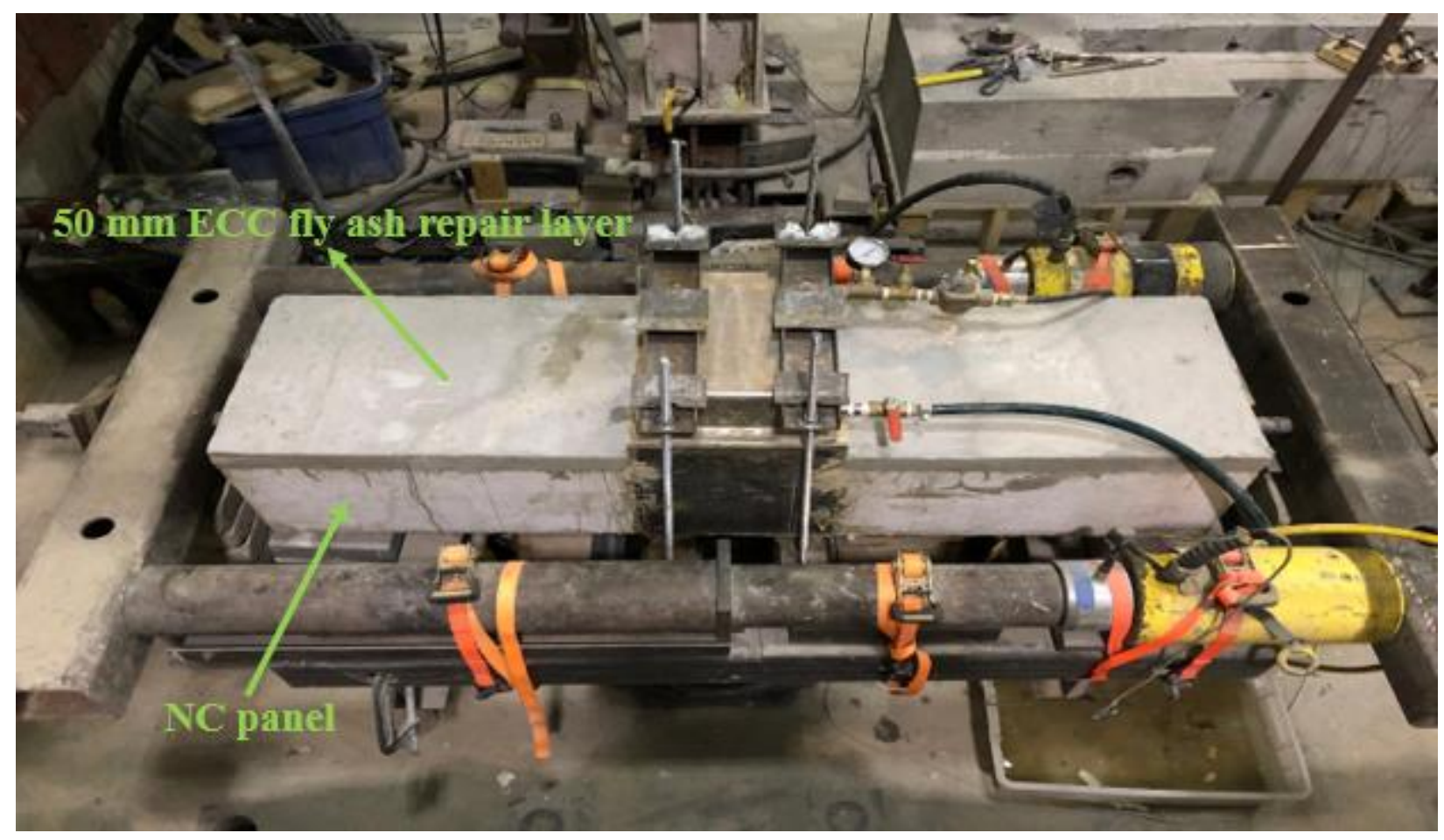

Figure 3-26: NC specimen repaired with a $50 \mathrm{~mm}$ layer of ECC fly ash 


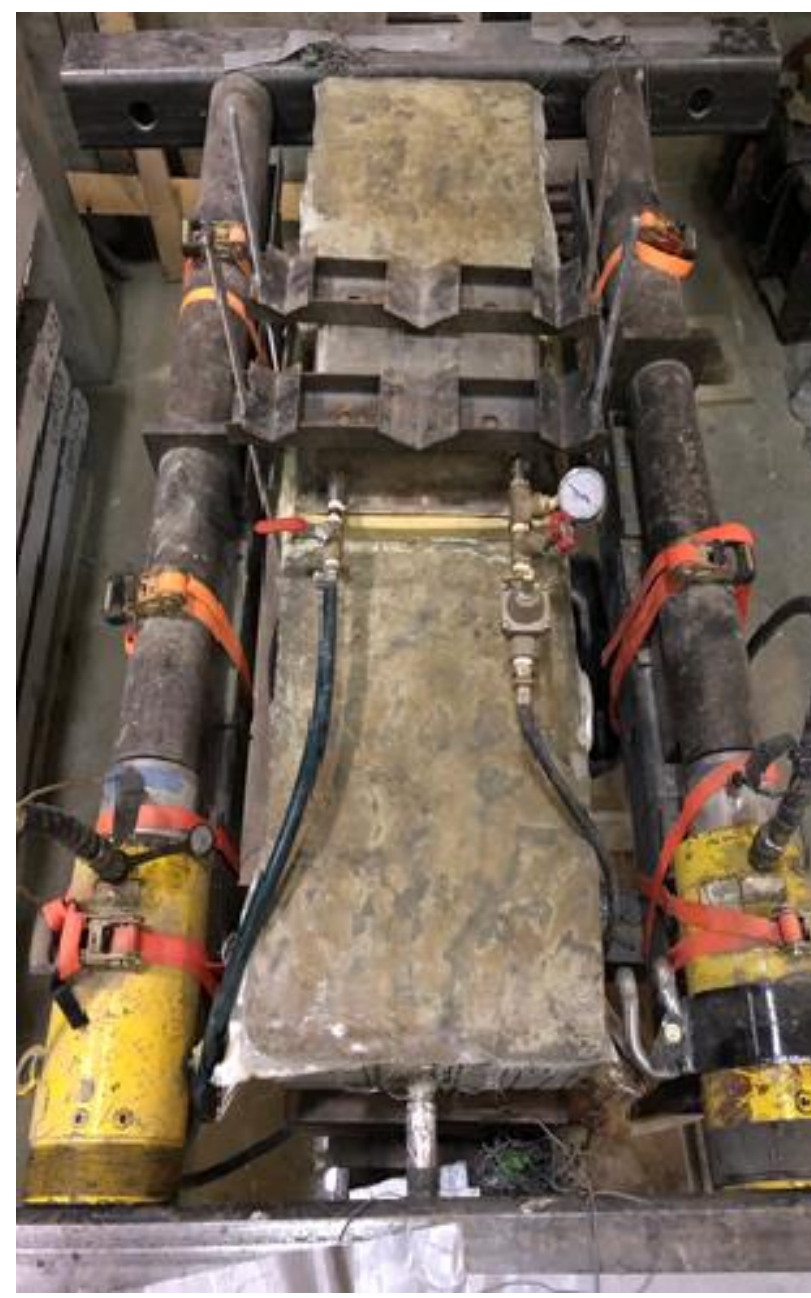

(a)

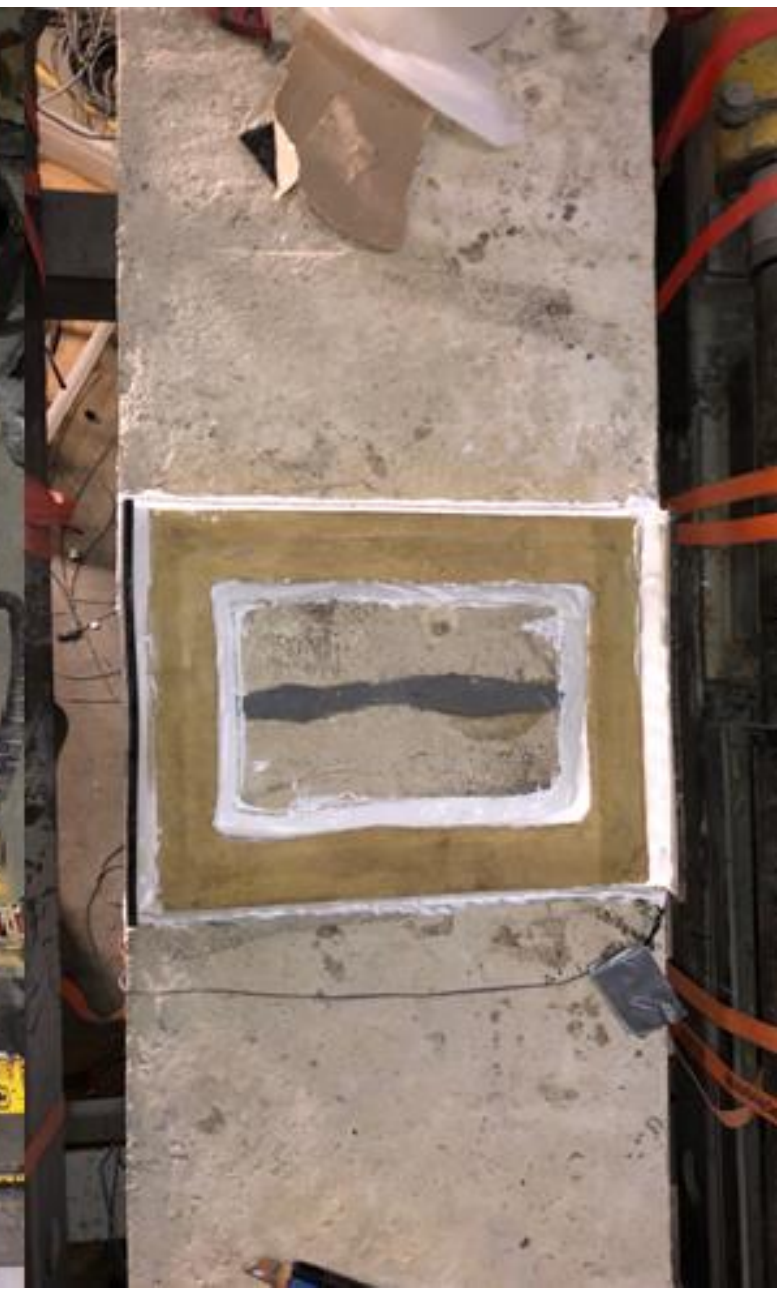

(b)

Figure 3-27: NC specimen repaired with a) GFRP laminates b) High-strength epoxy injection

Step 5: Self-healing test

After measuring the leakage rate of the repaired $\mathrm{NC}$ specimens under various water pressures, the pressure in the chamber is set to 7 psi to investigate the self-healing behavior of the NC specimens repaired with ECC slag and ECC fly ash under sustained tensile loading. For this purpose, the leakage rate is continuously recorded to achieve a constant leakage rate or $100 \%$ self-healing of the tension-induced cracks. Self-healing test is divided in to two parts including short-term selfhealing and long-term self-healing. In short-term investigation of the self-healing during the first 35 hours, the amount of water penetrating through the full depth of the tension cracks in ECC layer 
and NC panel and leaking from the bottom surface on the NC panel is measured continuously over a one-hour time intervals (each time for 60 minutes). In long-term investigation of the self-healing, during the first 35 hours leakage rate is recorded continuously each hour (each time for 60 minutes), while during the period of time between 35 hours up to 6 days (144 hours), leakage rate is measured for one hour duration in each 6 hours, followed by the last period of time from 6 days (144 hours) up to end of the test, leakage rate is recorded for one hour duration in each 8 hours.

\section{3-4-2 Procedure of applying the repair}

The assumption in this experimental program is to release the tensile load and remove water pressure chamber before applying the repair. This is to simulate the conditions in repair of a real water tank in which the tank is first emptied from water followed by applying the repair. Moreover, the repair is applied on top surface of the RC panels (where the water chamber is placed) to simulate applying the repair inside the water tank which is in contact with inside water.

\section{3-4-2-1 ECC slag and ECC fly ash repair layer}

The details about surface preparation, preparation of the form work and casting of the ECC slag and ECC fly ash repair layers on top of the NC panels were discussed in section 3-3. It is worth mentioning that the ECC repair layer is poured on top of the RC panel, where the water pressure is applied, which simulates casting the ECC repair layer inside the concrete water tank in real case.

\section{3-4-2-2 High-strength epoxy injection}

There are generally two methods of epoxy injection for a concrete cracked section namely highpressure and low-pressure epoxy injection. Low-pressure epoxy injection is easier to apply, cleaner, less expensive and requires less equipment and involvement compared to high-pressure 
injection. However, a crack as wide as around $1 \mathrm{~mm}$ is required to apply the low-pressure epoxy injection, which is much wider than the crack existing in water tanks. Crack width is a major concern in concrete structures that are designed under serviceability limit states such as water tanks and large crack width greatly threatens both functionality and durability of the liquid containing structures. Unless, a wide crack exists in the concrete section, use of low-pressure epoxy injection may results in filling only a short way into the crack which greatly affects the performance and efficiency of the epoxy. On the other hand, high pressure epoxy injection is more time consuming, and requires more equipment and labor cost. However, when high-pressure epoxy is performed properly, a more durable and reliable repair is achieved compared to low-pressure epoxy injection. Epoxy is forced to penetrate through the entire crack width by high-pressure pump resulting in producing a much finer pore structure in the cracked section and improving the bond between the epoxy and the concrete cracked surface.

In this experimental program, due to very low viscosity of the epoxy chosen for the repair, the possibility of low-pressure injection was evaluated at first. For this purpose, the specimen was reloaded up to $200 \mathrm{kN}$ to make a wider crack width. An average crack width of $0.5-0.55 \mathrm{~mm}$ was achieved at this level of loading. Despite the relatively large crack of width $0.5 \mathrm{~mm}$ and low viscosity of the epoxy, injection of the epoxy using low-pressure method was not possible. Therefore, high-pressure injection was performed at the same level of loading and crack width. The material properties of the high-strength low-viscosity epoxy used for injection was explained in section 3-2-3. High-pressure injection was performed in the following steps. At first, 3 holes with diameter of $3 / 8$ in were drilled inside the NC panel with different inclinations and lengths. It is worth-mentioning that the holes should pass through the cracked plane to ensure that the injected epoxy will fill the crack. The dust resulted from drilling the concrete was removed by using a 
vacuum cleaner as well as air compressor. The procedure of drilling the NC panel and removing the dust is shown in Figure 3-28.

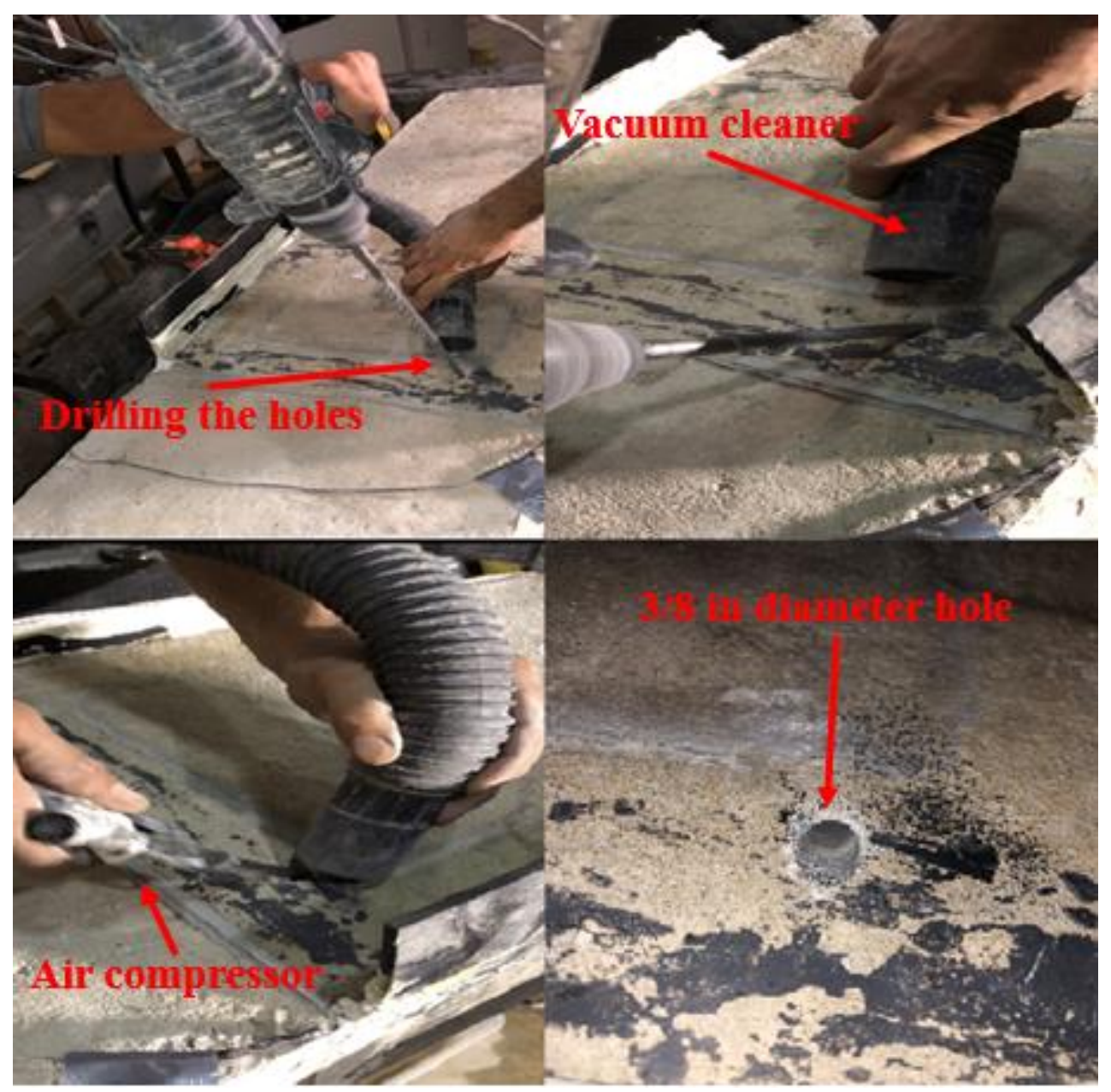

Figure 3-28: Procedure of drilling the NC panel and removing the dust using vacuum cleaner and air compressor

In the next step, injection ports were installed on top of the holes. Moreover, the top and bottom surfaces of the panel at cracked section were sealed using an epoxy sealer to form a high-build protective layer on the concrete surface and prevent dripping during the high-pressure epoxy injection. There was no need to seal the sides of the panel as both sides were already sealed using waterproof membrane sheets and a water-resistant adhesive for the leakage test. Figure 3-29 illustrates installing the ports on top of the holes and sealing the bottom surface of the concrete panel at the crack location. 


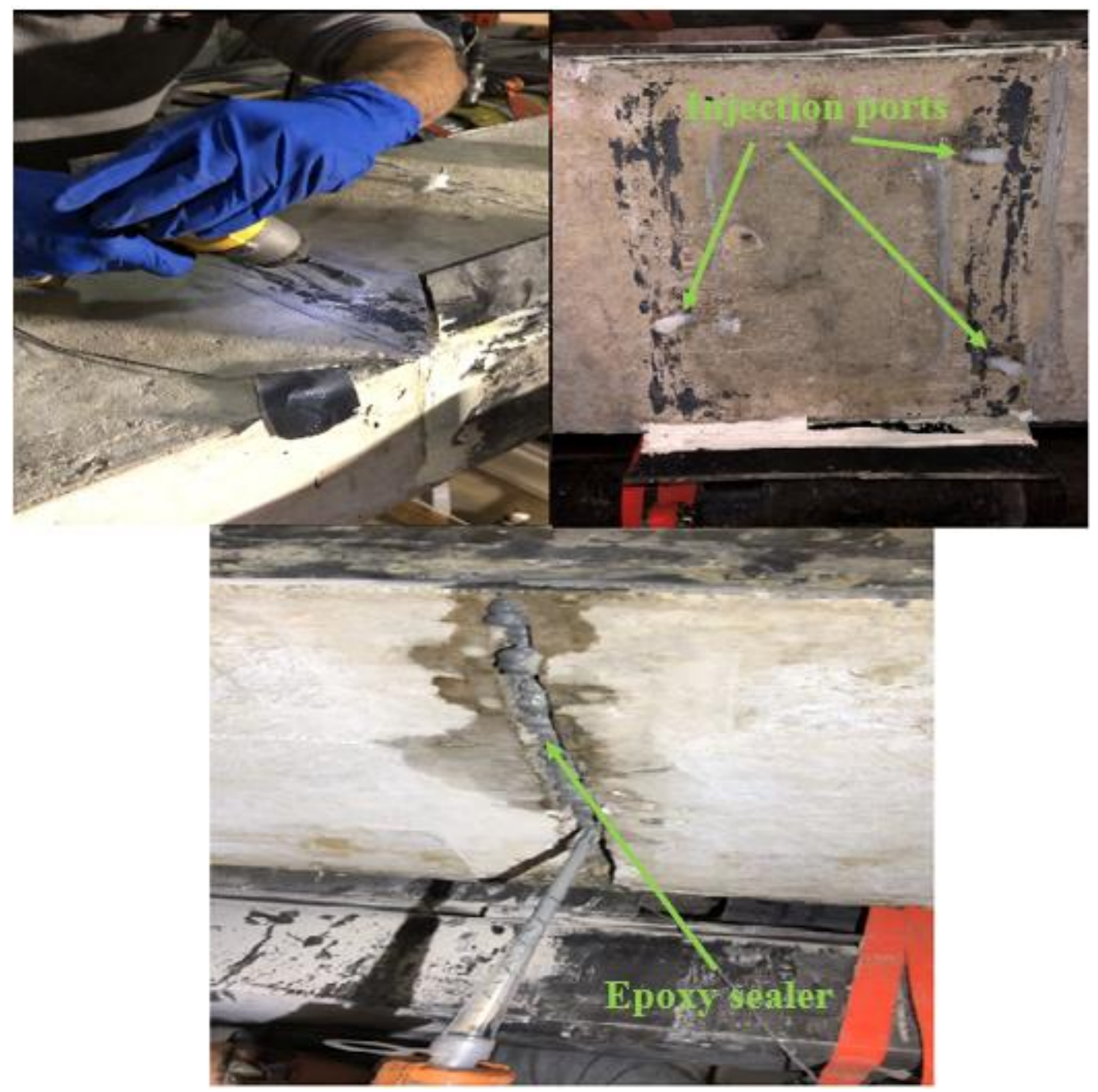

Figure 3-29: Installation of the injection ports on top of the holes and sealing the bottom surface of the NC panel at crack location

Next, epoxy was injected using a high-pressure pump reaching 1000 psi to ensure epoxy filled the entire depth of crack, which is shown in Figure 3-30.

After performing the high-pressure epoxy injection, the tensile load was released which was followed by dripping a few amount of epoxy indicating that the entire depth of the crack was filled with epoxy (residual crack width of $0.22 \mathrm{~mm}$ ). Then the epoxy was let to cure for two weeks to gain high-strength specified in the data-sheet including high tensile and bond strength. The 
finished surfaces of the NC panel at top and bottom after the injection of the epoxy are shown in Figure 3-31.

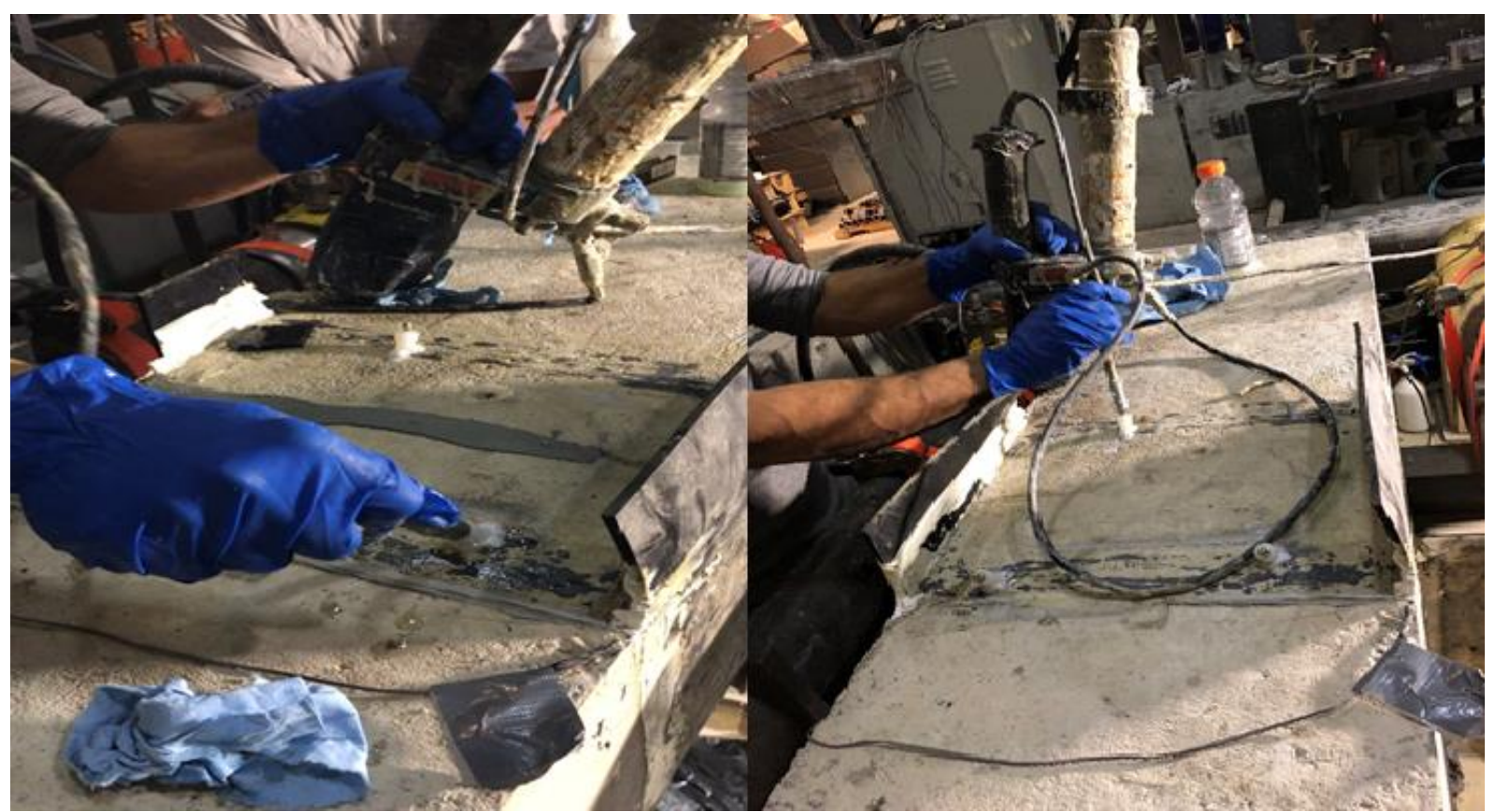

Figure 3-30: High-pressure epoxy injection

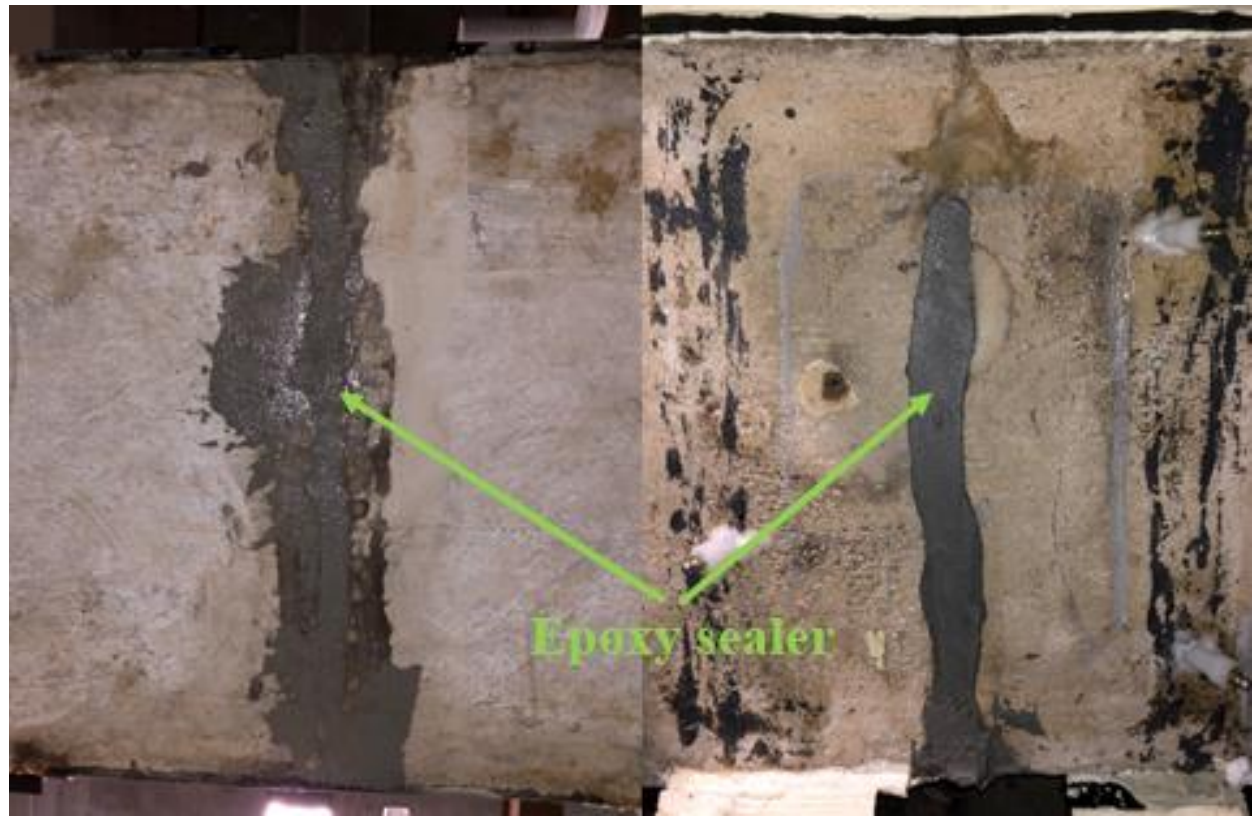

(a)

(b)

Figure 3-31: Finished surfaces of the NC panel after high-pressure epoxy injection a) Bottom surface b) Top surface 


\section{3-4-2-3 Glass fiber reinforced polymer (GFRP) laminate}

Glass fiber reinforced polymer (GFRP) laminate was used for this experimental program to repair the NC panels. A unidirectional E-glass type of fiber fabric was used in conjunction with epoxy laminating resin to provide composite strengthening system. Material properties of the E-glass fiber, epoxy resin system, and cured GFRP laminate were discussed in section 3-2-4. It is worthmentioning that the GFRP laminate was applied on the top surface of the RC panel, where the water pressure is applied, to simulate repairing a water tank by applying the GFRP laminate inside it. Applying the GFRP laminate performed in the following steps. The first step which greatly affects the quality of the repair program is surface preparation. It is common practice to prepare the concrete surface by sandblasting to expose the coarse and fine aggregates according to ACI 546R (2014). The surface of the RC panel was ready enough for proper applying of the GFRP without the need for sandblasting. In the next step, all the dust was removed from the concrete surface using a vacuum cleaner and air compressor. Moreover, the concrete surface must be free

from grease and oil and dry with maximum surface moisture less than $4 \%$ by weight. Next, the evenness of the RC panel surface was measured by placing a straight edge against the concrete surface. This is an important point to note the uneven surface will result in failure of the GFRP laminate by premature peeling under loading. The prepared surface of the RC panel is shown in Figure 3-32. 


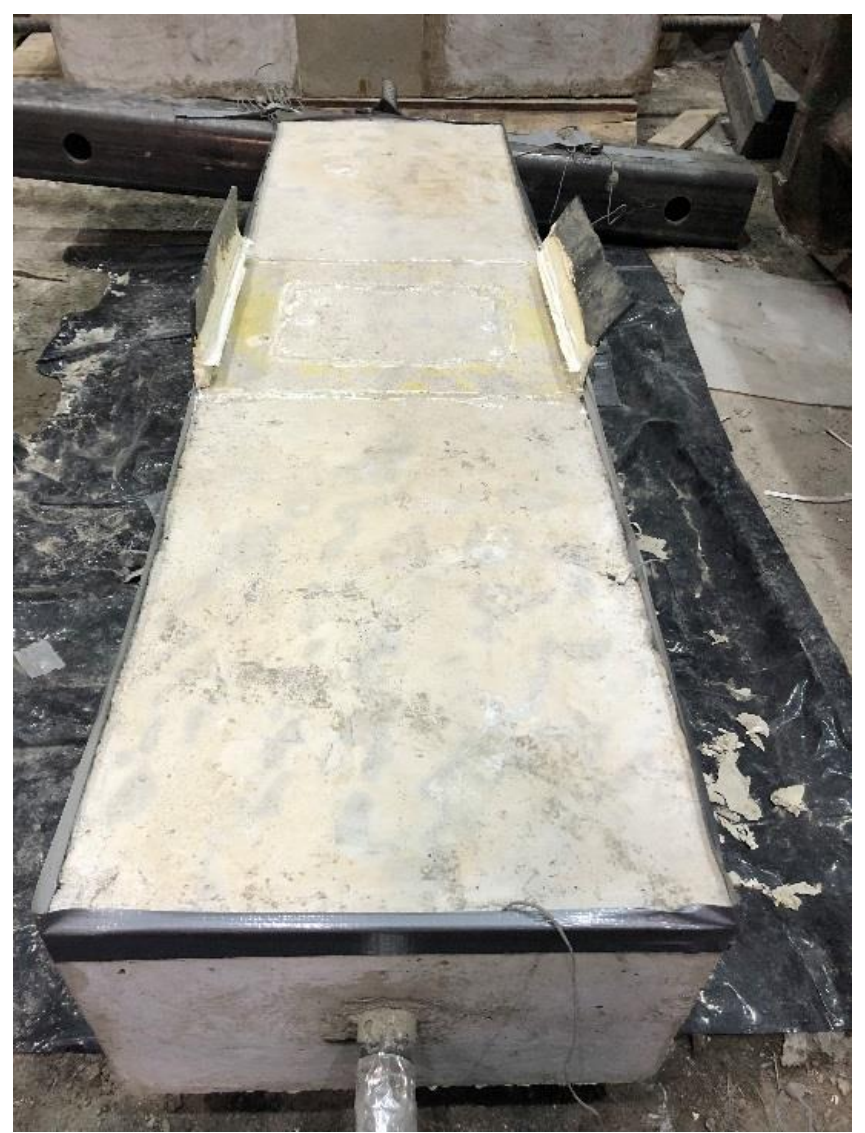

Figure 3-32: Prepared surface of the RC panel for applying GFRP

Next, the glass fiber fabrics were wiped out with acetone to remove any other excess residue from it. In the next step, resin and slow hardener were mixed together properly and applied on the entire concrete surface using a painting brush, which illustrated in Figure 3-33.

In order to prevent the failure of the GFRP laminate and prevent the leakage after repair, and based on the tensile strength of the cured GFRP laminate and nominal laminate thickness and also by considering the maximum load that can be applied on the RC panel, it was decided to use two layers of GFRP. The first layer of GFRP was placed carefully on the surface of the RC panel and pressed in place using a hard rubber roller to remove any possible voids between the GFRP layer and concrete surface. Epoxy resin was applied again and second layer of GFRP was placed followed by applying the epoxy resin using the painting brush. Figure 3-34 shows the finished surface of the RC panel after applying the GFRP. 


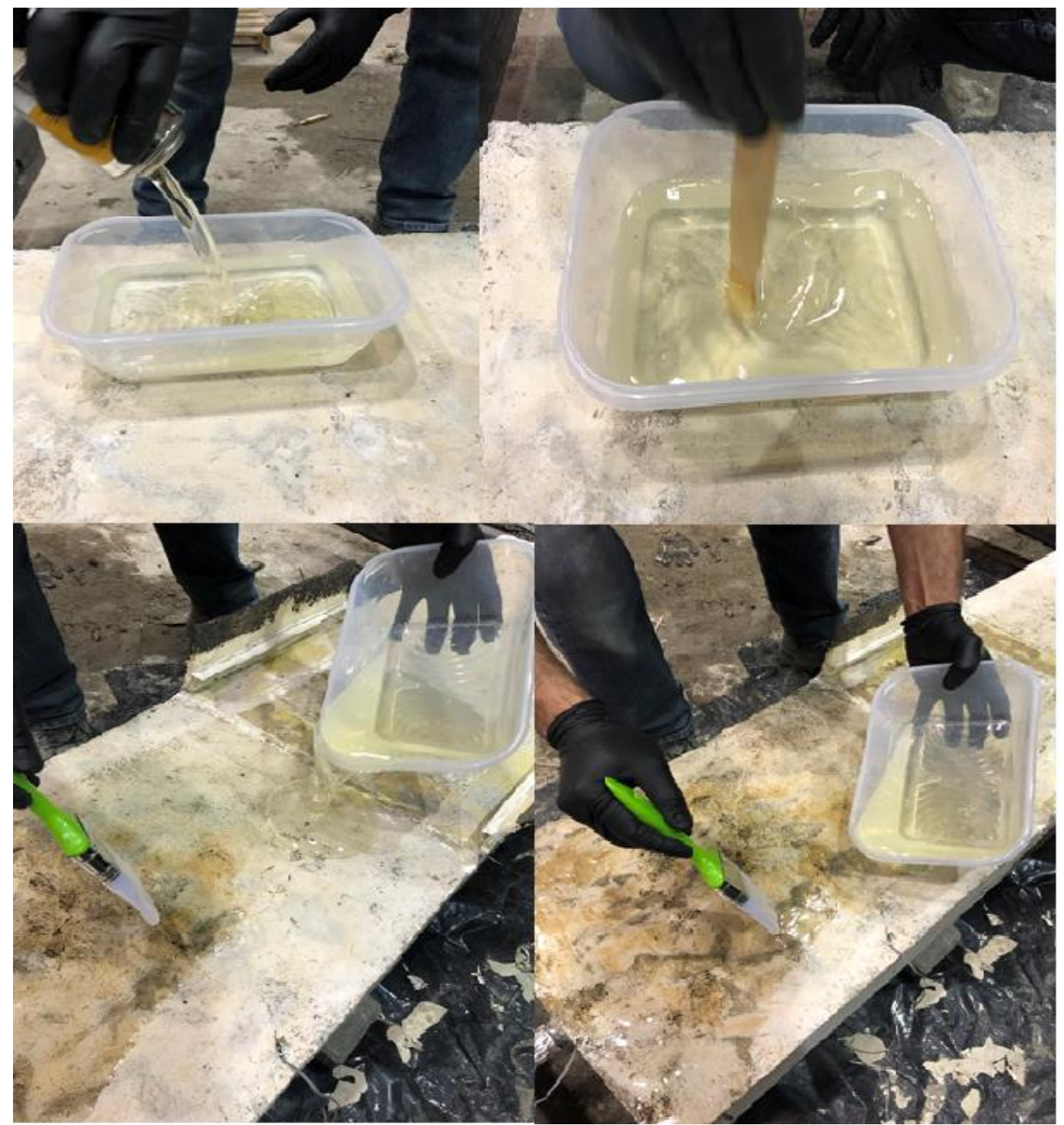

Figure 3-33: Mixing the resin and hardener and applying the epoxy resin system on the top surface of the RC panel 


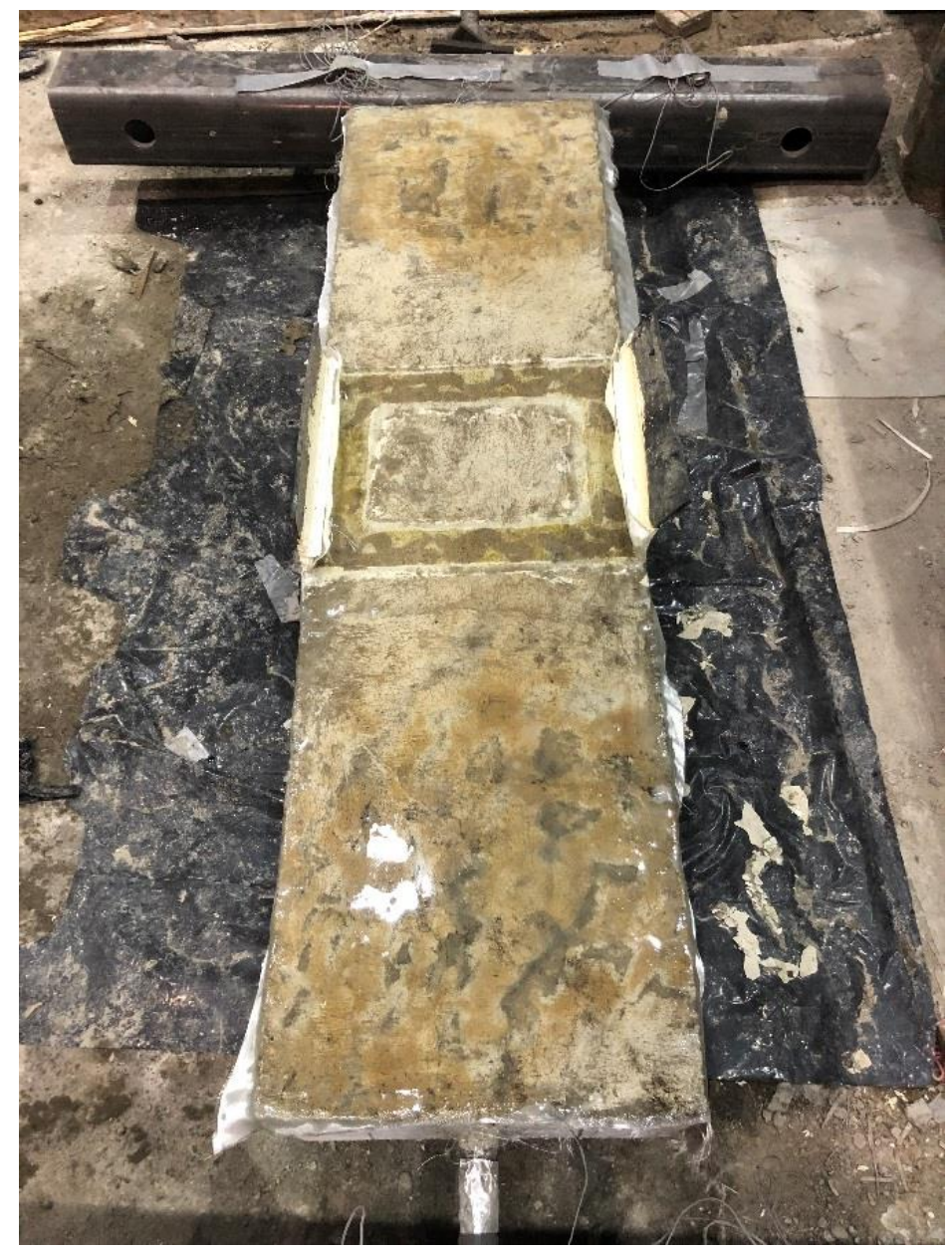

Figure 3-34: Finished surface of the RC after applying the GFRP 


\section{Chapter Four: Results and Discussion}

\section{4-0 Introduction}

The results of the experimental program on the repair of RC panels using Engineered Cementitious Composite (ECC) made with slag, Engineered Cementitious Composite (ECC) made with fly ash, high-strength epoxy, and Glass Fiber Reinforced Polymer (GFRP) laminates are presented in this chapter. A total number of 4 full-scale RC panels made with normal concrete (NC) were tested under monotonic tensile load to induce tension cracks at pre-determined locations at the middle of the specimens. In the next step, RC samples were repaired using the above-mentioned materials. The load at which crack initiated, crack width, leakage rate under different water pressures, and self-healing of the NC and ECC samples were recorded before and after the repair. The decision variables in this experimental program included type of the repair materials (ECC slag, ECC fly ash, high-strength epoxy, GFRP laminate). The results of the tests are presented by discussing the load versus displacement, strain development in the steel rebar, crack development and crack width, leakage rate under various water pressures, and self-healing of the NC sample and ECC repair layer. The efficiency of the repair materials is compared in terms of strength, crack development, and leakage control, and self-healing capability.

\section{4-1 Performance of $\mathrm{RC}$ panels before repair}

Performance of the 4 full-scale RC panels made with NC (NC1, NC2, NC3, NC4) under monotonic tensile loading is described and compared. 


\section{4-1-1 Crack width and strength of the $\mathrm{RC}$ panels}

RC panels made with $\mathrm{NC}$ were tested under monotonic tensile loading according to the test procedure and test setup outlined in Chapter 3. Tensile load was applied using the hydraulic jacks to create a full-depth crack at the middle of the specimens, where the notch was placed. Brittle nature of NC resulted in formation of a full depth crack followed by high rate of leakage. Sudden cracking of $\mathrm{NC}$ panel along with simultaneous strength failure and leakage initiation indicate how brittle and catastrophic regular RC panels could be in terms of serviceability performance. The load at leakage initiation and the corresponding crack width under direct tensile load as well as the residual crack width in NC panels after releasing the load are presented in Table 4-1. The load at which leakage started varied from $114 \mathrm{kN}$ to $127 \mathrm{kN}$. NC4 exhibited the highest load of $127 \mathrm{kN}$, while the lowest load at leakage initiation of $114 \mathrm{kN}$ occurred in NC2. All NC specimens experienced average crack width in the range of 0.3 to $0.4 \mathrm{~mm}$, which provided almost the same condition before repair for all samples and helped to make a better comparison in terms of the efficiency of the repair materials. Figure 4-1 shows NC specimen mid-span crack at bottom and side surfaces. 


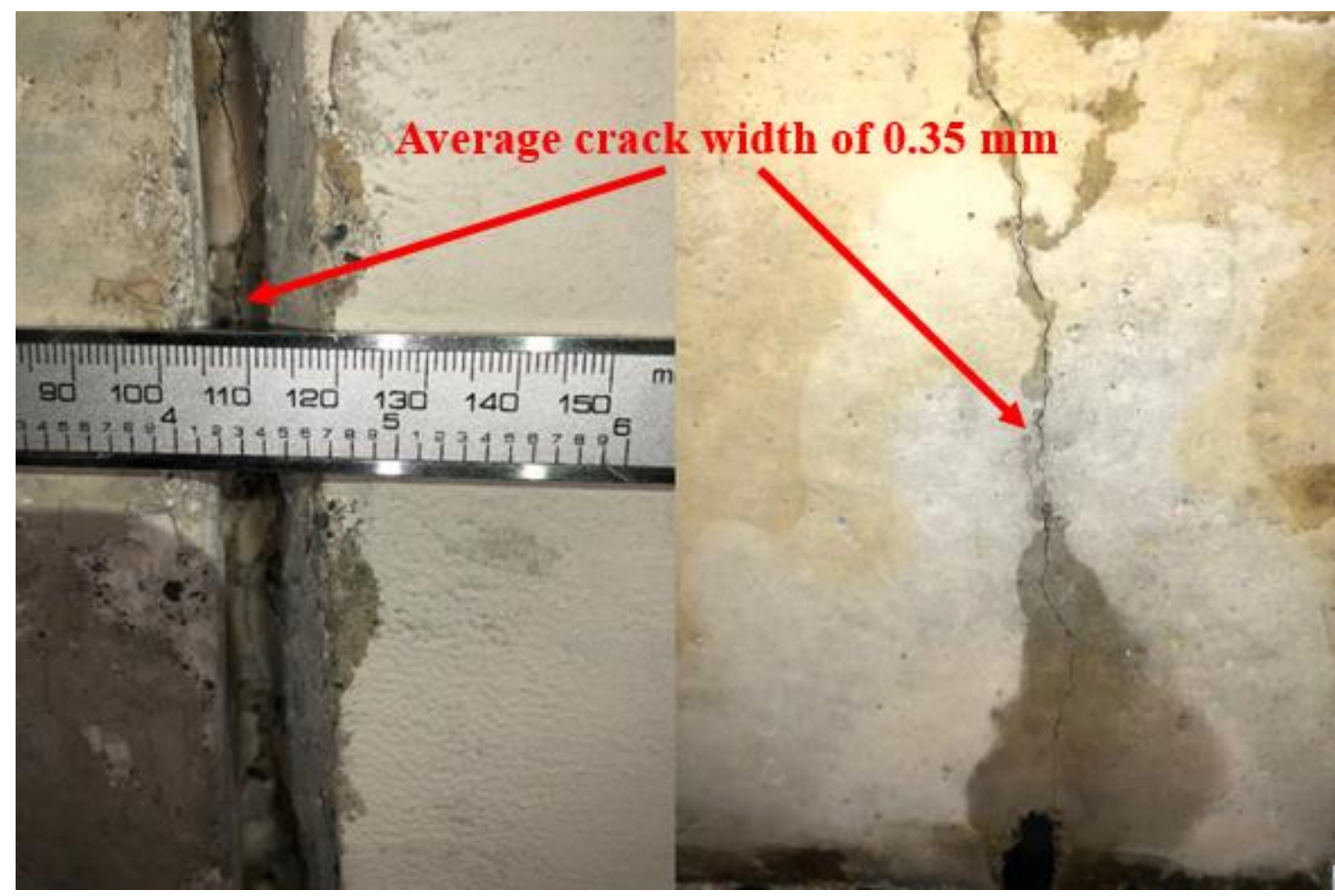

(a)

(b)

Figure 4-1: NC specimen cracked at mid-span a) Bottom face b) Side face

Table 4-1: Load at leakage initiation and average crack width of NC specimens

\begin{tabular}{|c|c|c|c|}
\hline Specimen & $\begin{array}{c}\text { Load at leakage initiation } \\
(\mathrm{kN})\end{array}$ & $\begin{array}{c}\text { Crack width } \\
(\mathrm{mm})\end{array}$ & $\begin{array}{c}\text { Residual crack width } \\
(\mathrm{mm})\end{array}$ \\
\hline $\mathrm{NC} 1$ & 125 & $0.3-0.35$ & 0.11 \\
\hline $\mathrm{NC} 2$ & 114 & 0.3 & 0.07 \\
\hline $\mathrm{NC} 3$ & 118 & $0.3-0.35$ & 0.09 \\
\hline $\mathrm{NC} 4$ & 127 & $0.35-0.4$ & 0.13 \\
\hline
\end{tabular}

\section{4-1-2 Strain development and load-displacement response of the NC panels}

Three steel strain gauges were placed on the single rebar, one in the middle of the sample (notch location), and two other steel strain gauges at 1/6 of the span from each end of the specimen, as shown in Figure 4-2. 


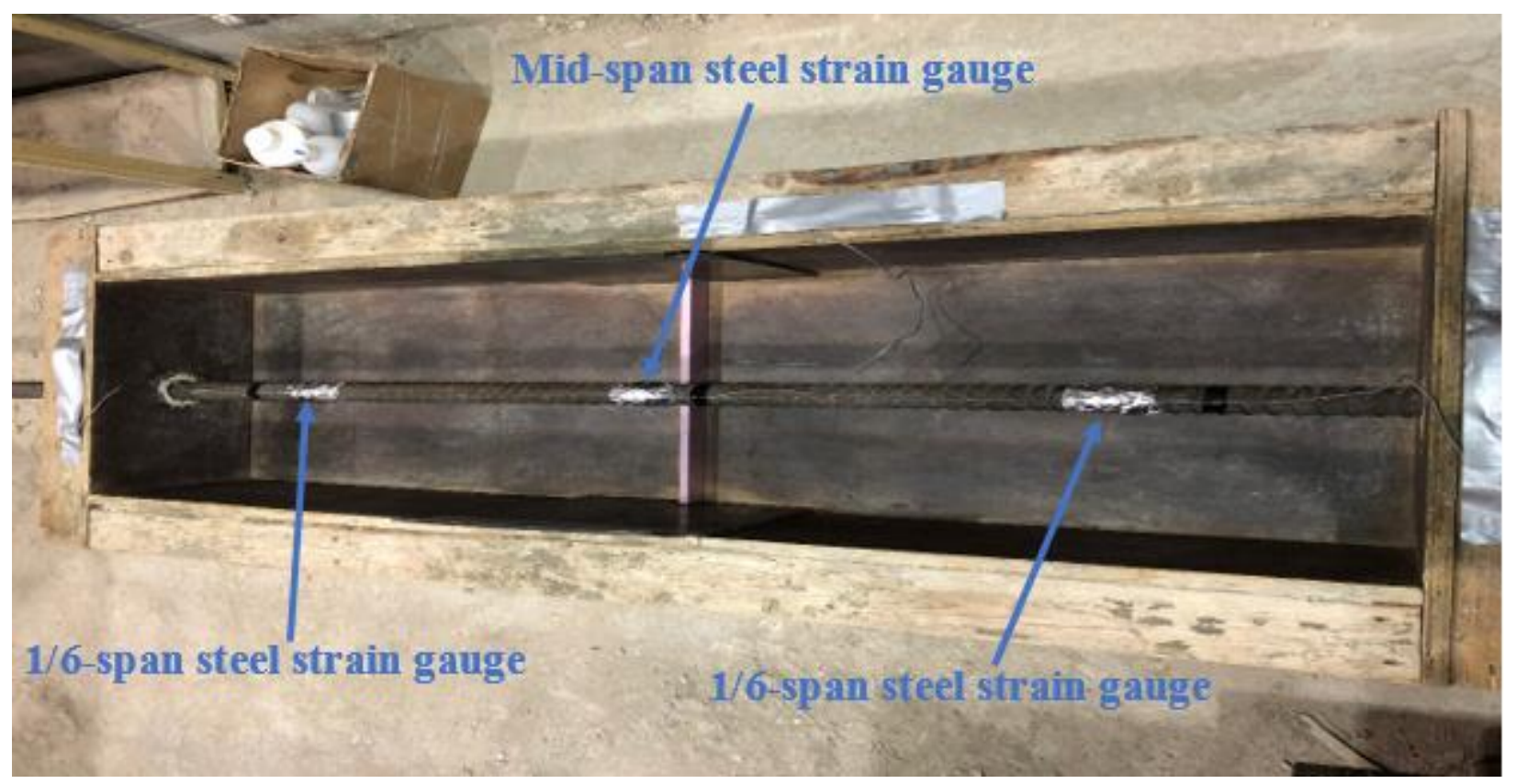

Figure 4-2: Arrangement of steel strain gauges on the rebar

Figures 4-3 (a-d) show the development of tensile steel strain at various locations of the specimens. As expected, the tensile train increased in the rebar gradually by applying the monotonic tension loading. At this stage of loading concrete section is uncracked and still capable of carrying the tensile load. Therefore, applied tensile load is transferred to the concrete through the bonding between the rebar and concrete. This was followed by a sudden jump of the steel strain (at midspan) at cracking load of the NC specimens. This can be explained by the fact that after cracking of the concrete section, it is no longer able to carry the tensile load and consequently rebar has to carry all the tension at cracked section at mid-span, where the crack occurred. Since the loading stopped at this stage, no cracking was developed at other locations of the span and that represents the absence of any jump in steel strain at other steel strain gauges. As designed, the tensile strain in the rebar did not reach the yielding strain before the repair in any of the NC specimens. The reason for keeping the rebar strain far less than the yielding strain of the rebar is to prevent possible yielding of the rebar before the repair and to allow the specimens to reach higher levels of loading 
after being repaired. The maximum tensile strain occurred at mid-span (crack location) in all NC samples ranged between 750 and 851 micro-strain, which were all less than half of the steel yielding strain with maximum of 851 micro-strain in NC4 and minimum of 750 micro-strain in $\mathrm{NC} 2$.

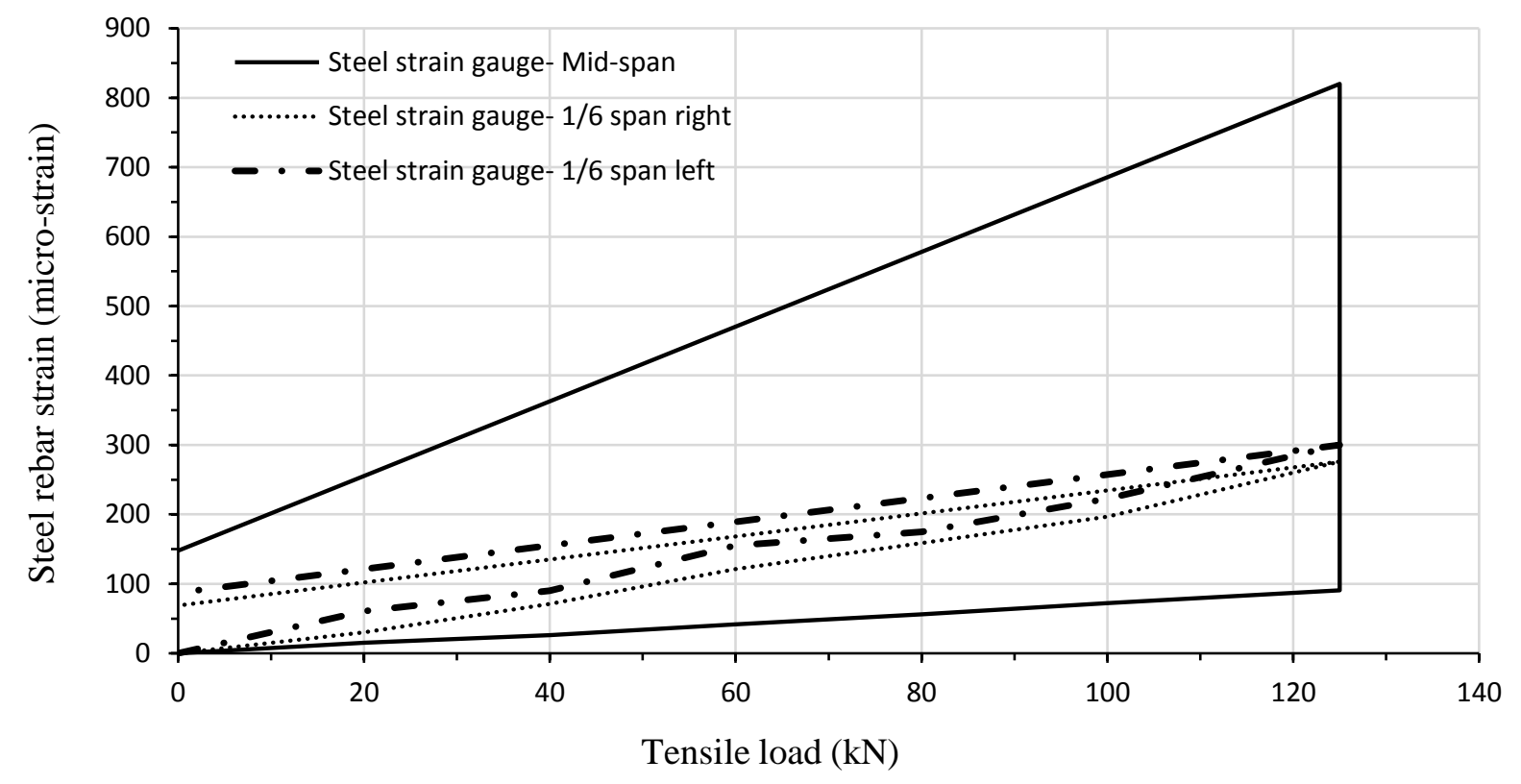

(a) $\mathrm{NC1}$ 


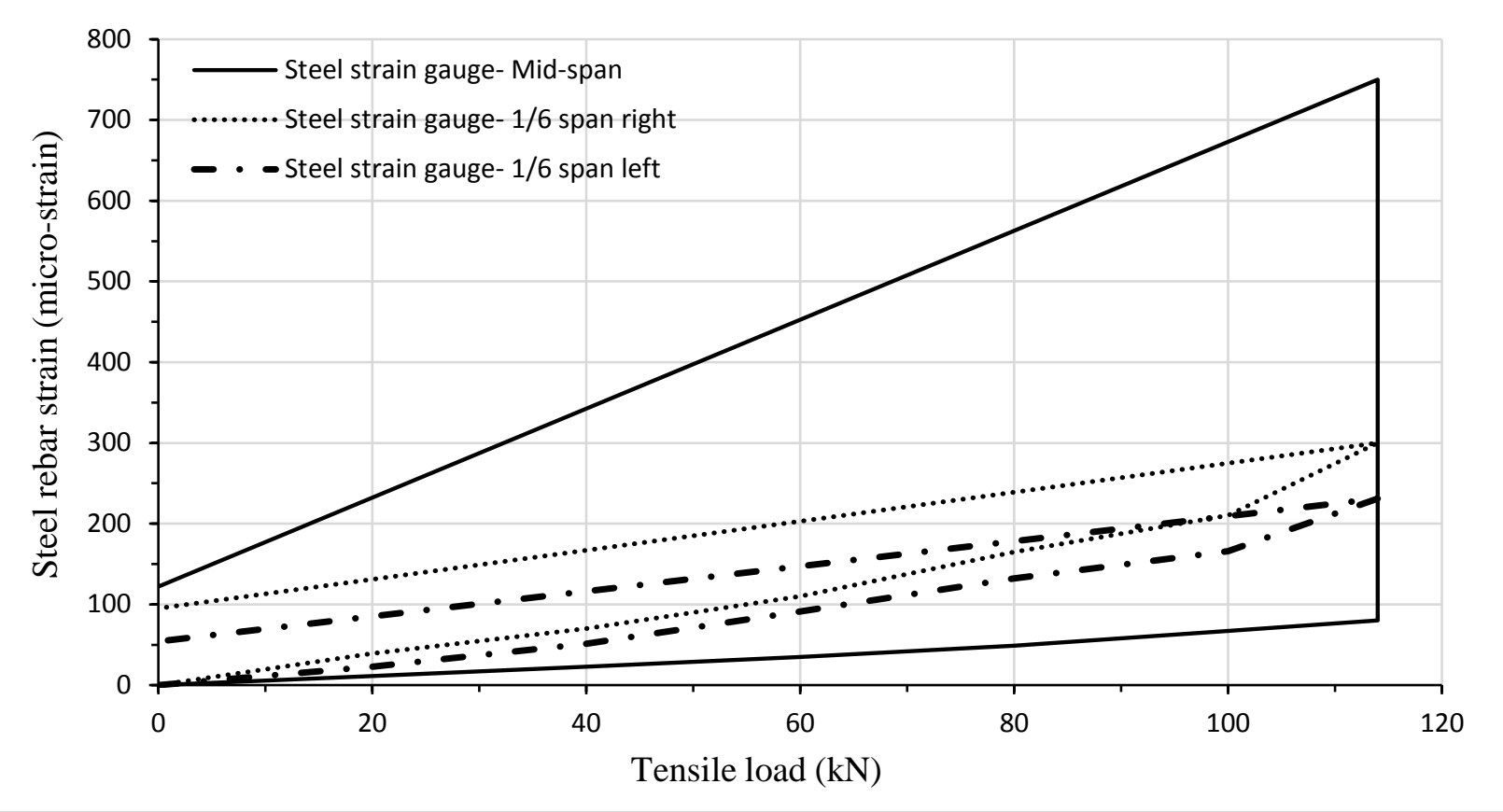

(b) $\mathrm{NC} 2$

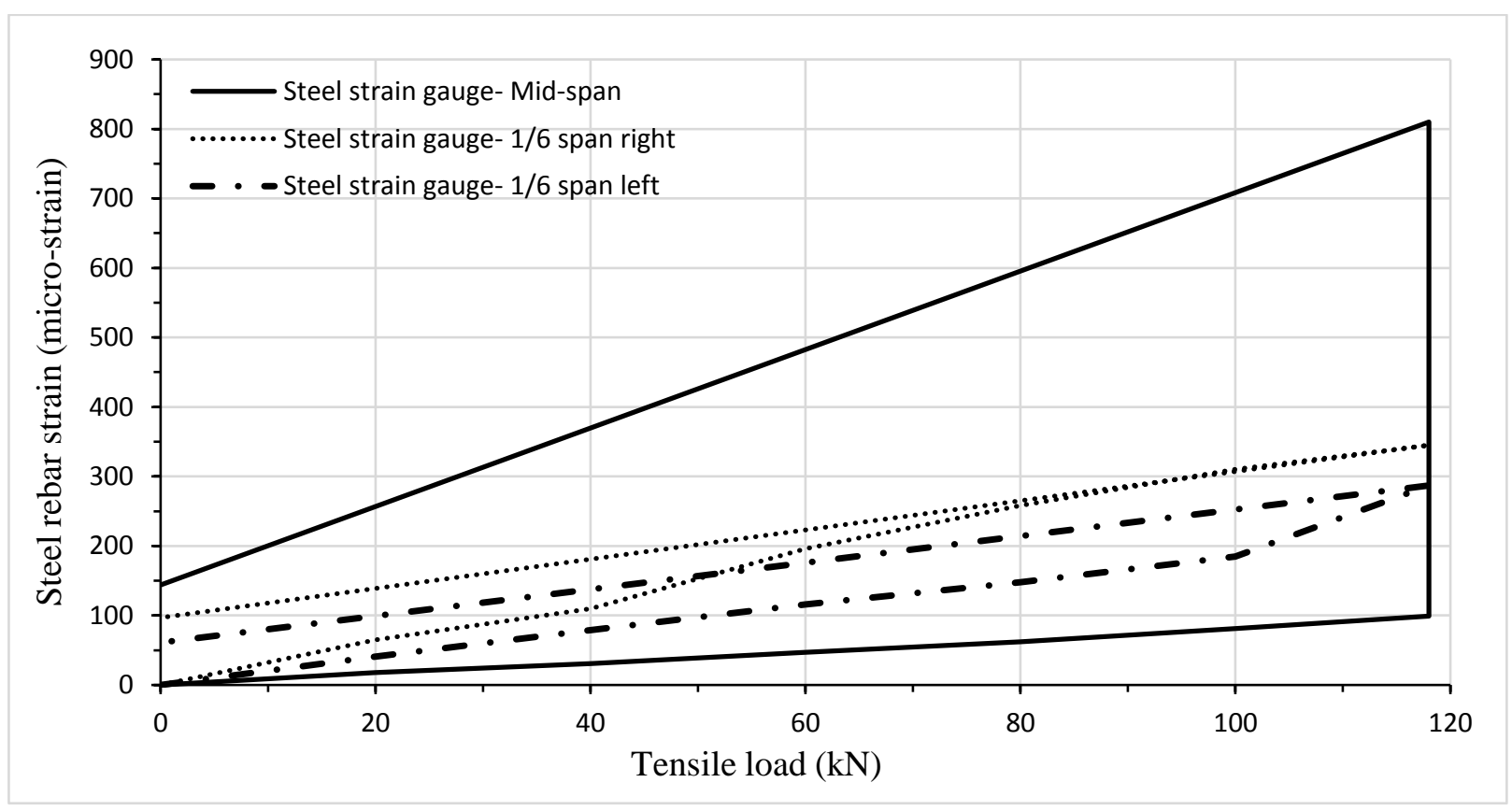

(c) $\mathrm{NC} 3$ 


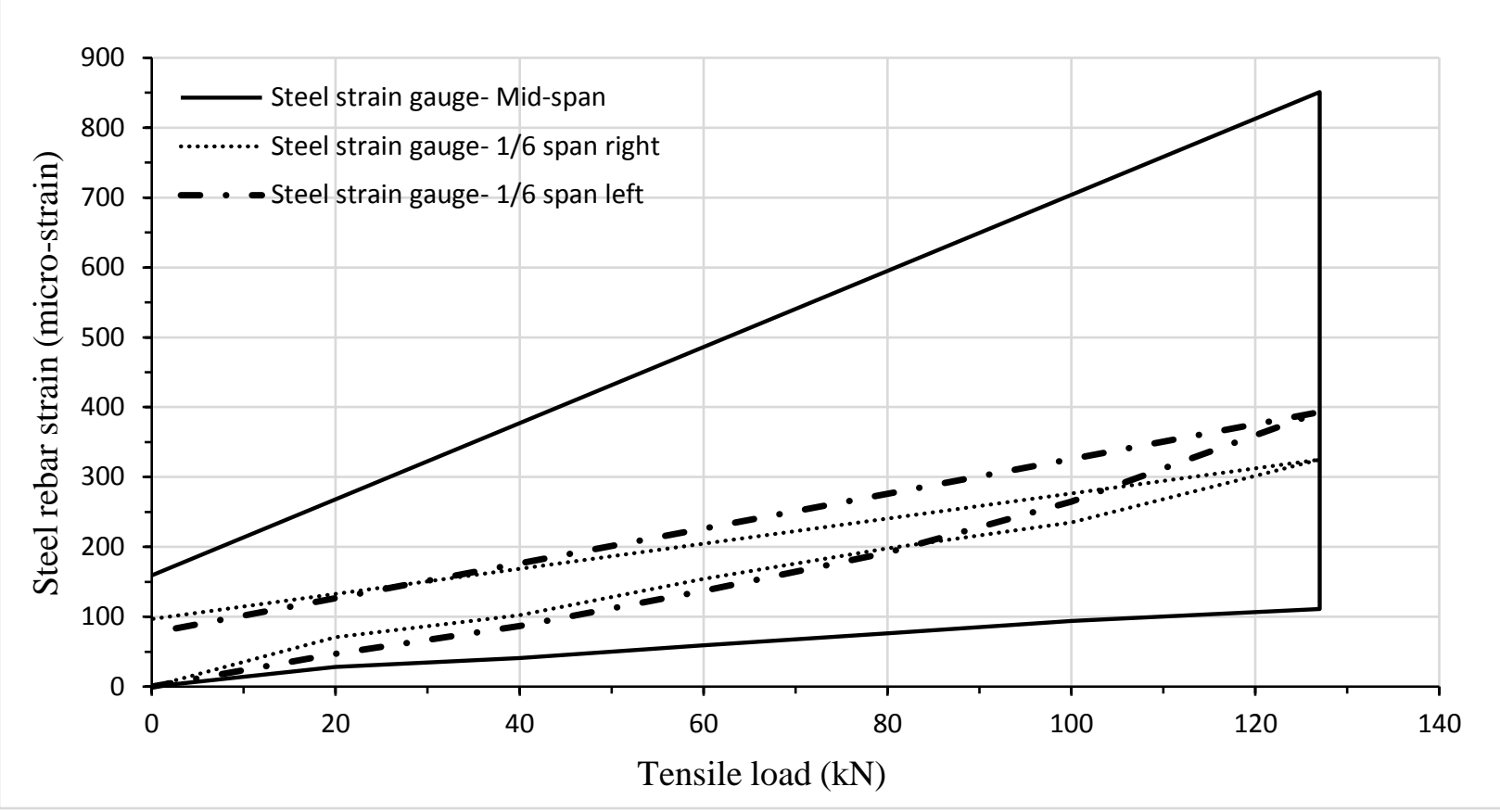

(d) $\mathrm{NC4}$

Figure 4-3: Steel strain development at typical strain gauge in a) NC1 b) NC2 c) NC3 d) NC4 (before repair)

Two LVDTs were attached to the sides of the sample at mid-span to measure the horizontal displacement of the NC specimens with respect to the mid-span (crack location), representing the mid-span crack width, as shown in Figure 4.4. Figures 4-5 (a-d) illustrate the load versus relative horizontal displacement of the NC specimens. The horizontal displacement does not change significantly by applying the tensile load to the specimen. However, a sudden jump can be observed in the load displacement responses at the cracking load of the specimens. This can be explained by brittle cracking of the concrete section at the weakest section (mid-span) resulting in a sharp increase in the horizontal displacement of the samples. The maximum horizontal displacement of the specimens varied from $0.28 \mathrm{~mm}$ to $0.38 \mathrm{~mm}$. The lowest horizontal displacement belonged to NC2 with $0.28 \mathrm{~mm}$ at the load of $112 \mathrm{kN}$ (mid-span crack width of 0.3 $\mathrm{mm}$ ), while NC4 experienced the highest horizontal displacement of $0.38 \mathrm{~mm}$ at the load of 127 $\mathrm{kN}$ (mid-span crack width of 0.35-0.4 mm). The residual horizontal displacement in specimens 
after releasing the load ranges between 0.1 to $0.15 \mathrm{~mm}$ which is an indication of the residual crack in $\mathrm{NC}$ panels (residual cracks ranged between 0.07 to $0.13 \mathrm{~mm}$ ).

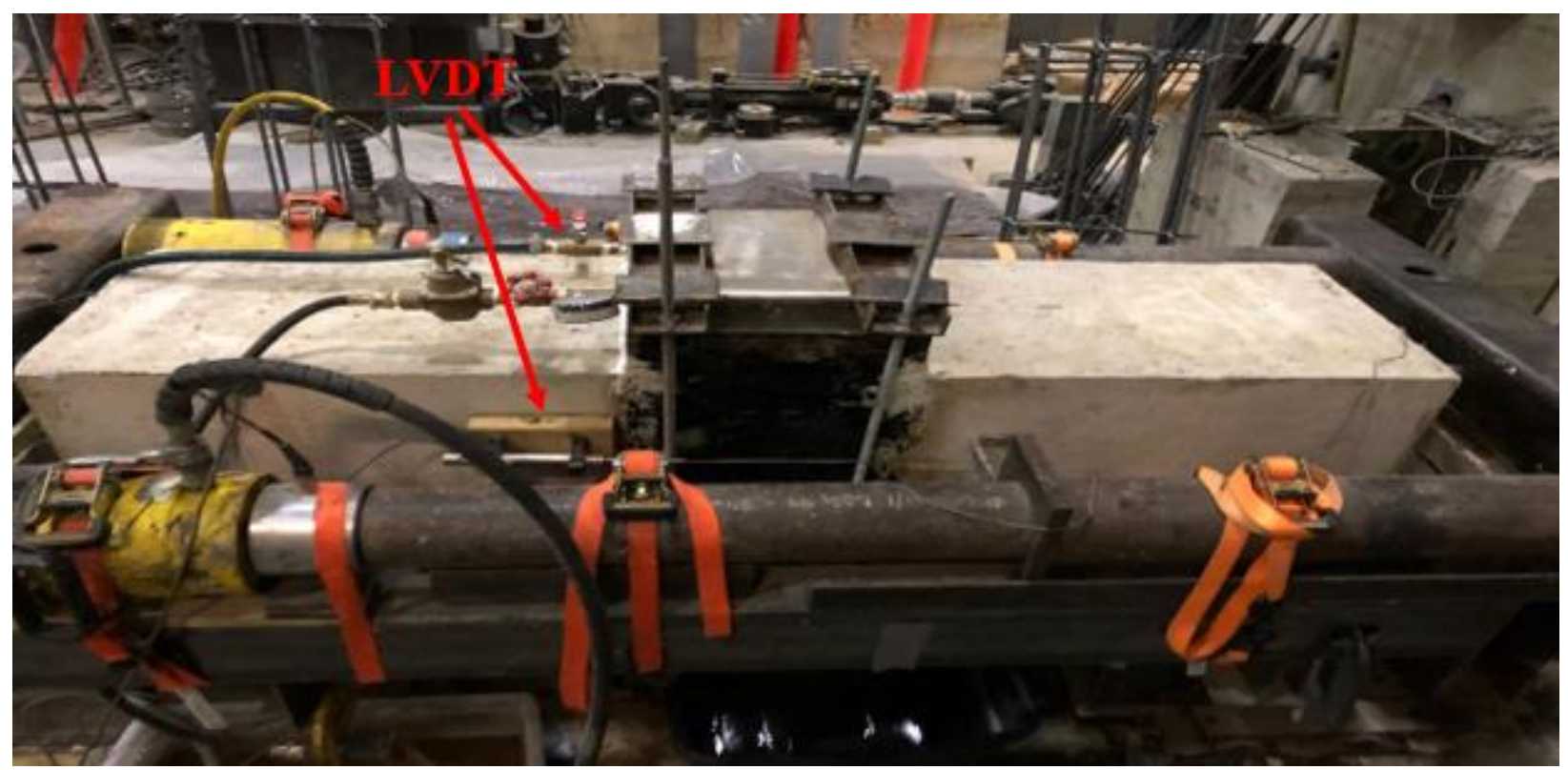

Figure 4-4: Locations of LVDTs at the middle of the specimen

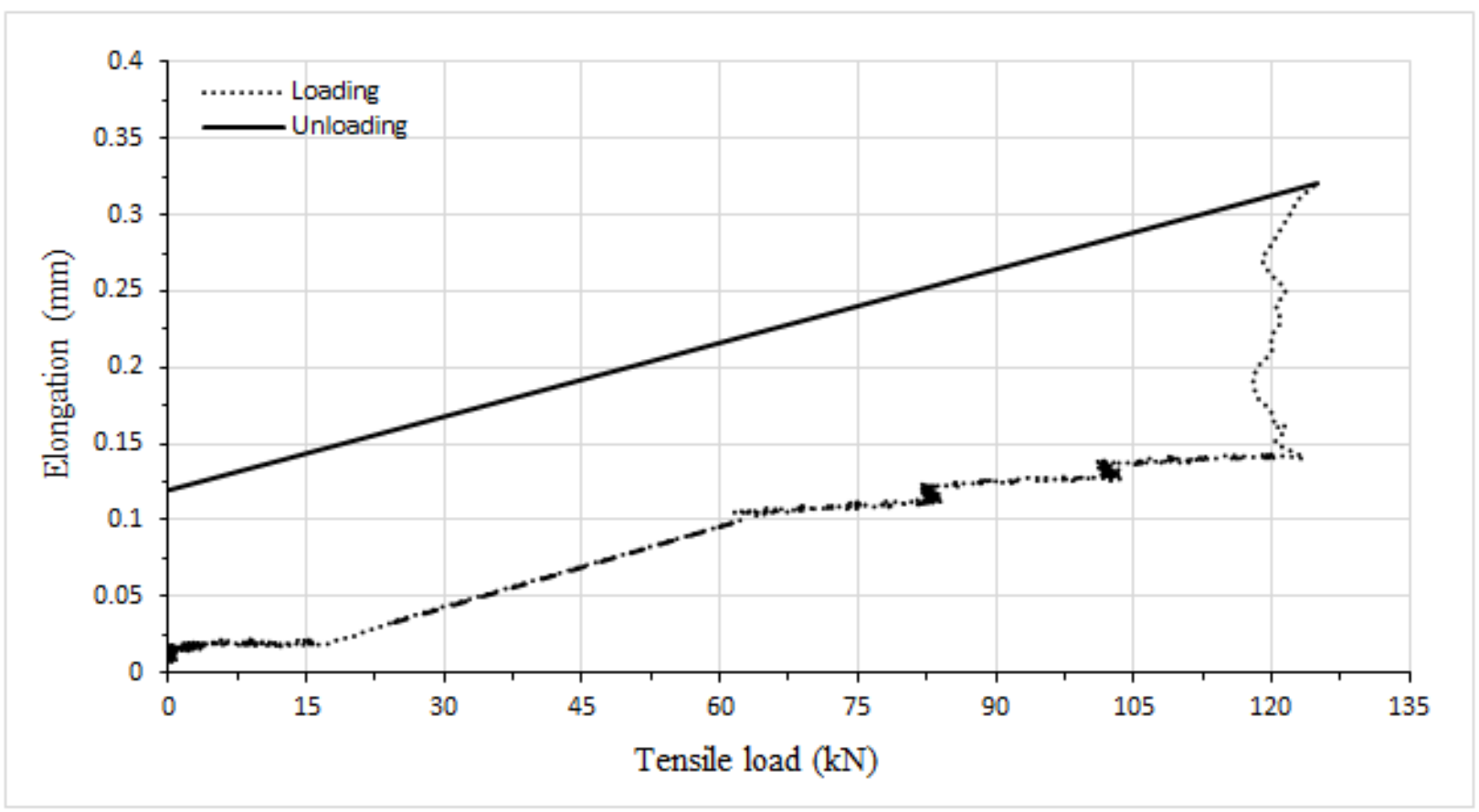

(a) $\mathrm{NC1}$ 


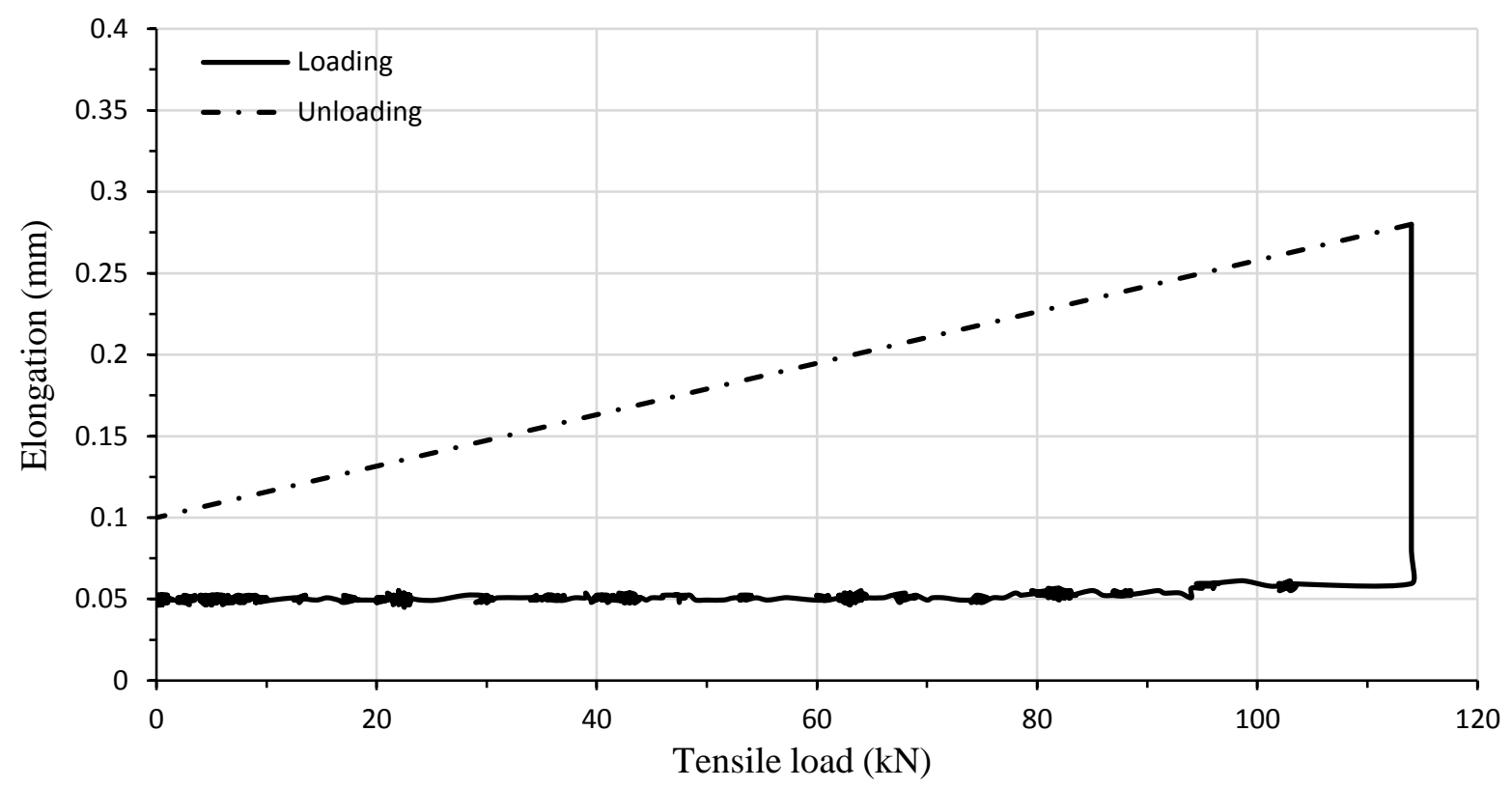

(b) $\mathrm{NC} 2$

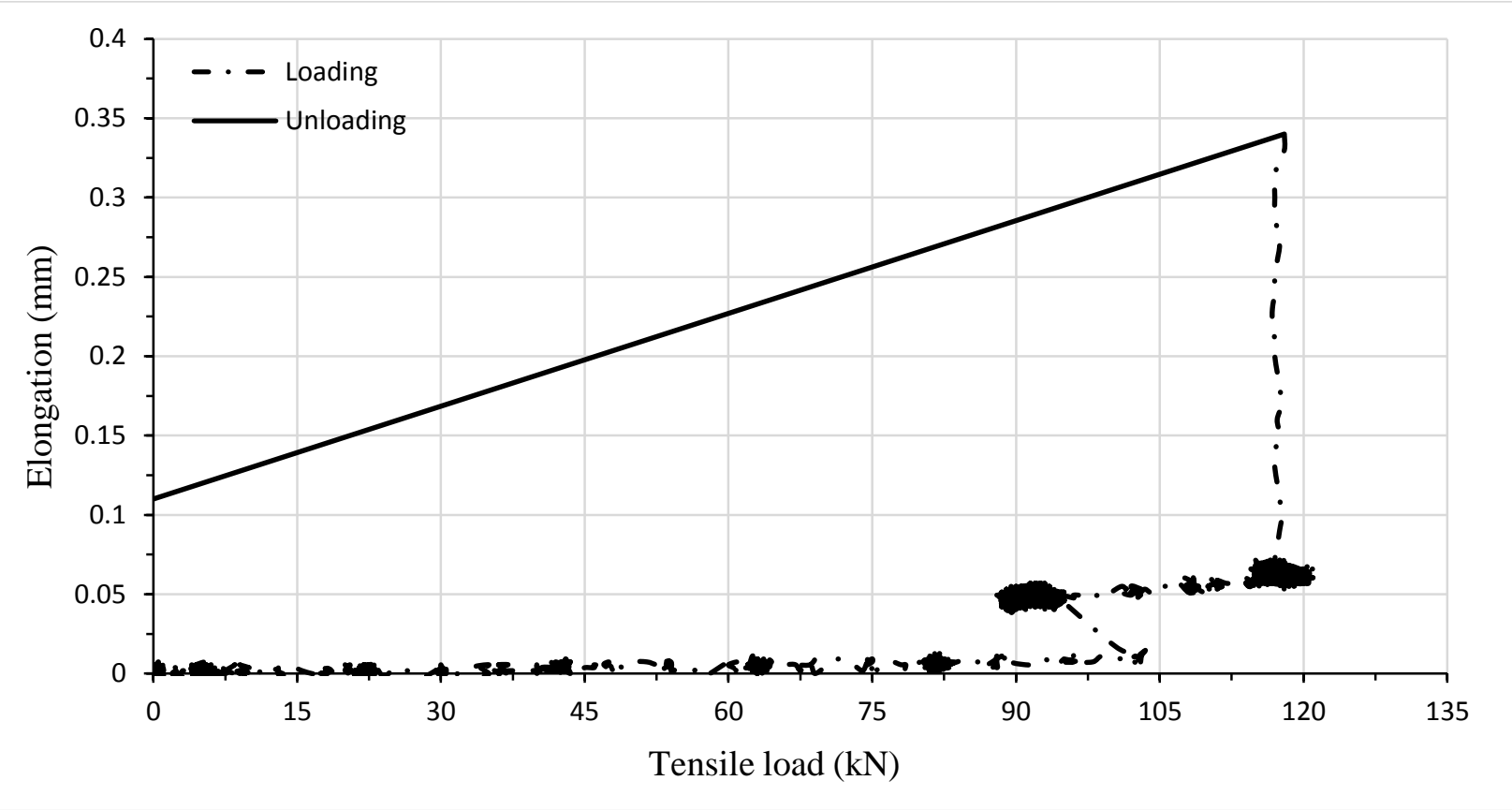

(c) $\mathrm{NC} 3$ 


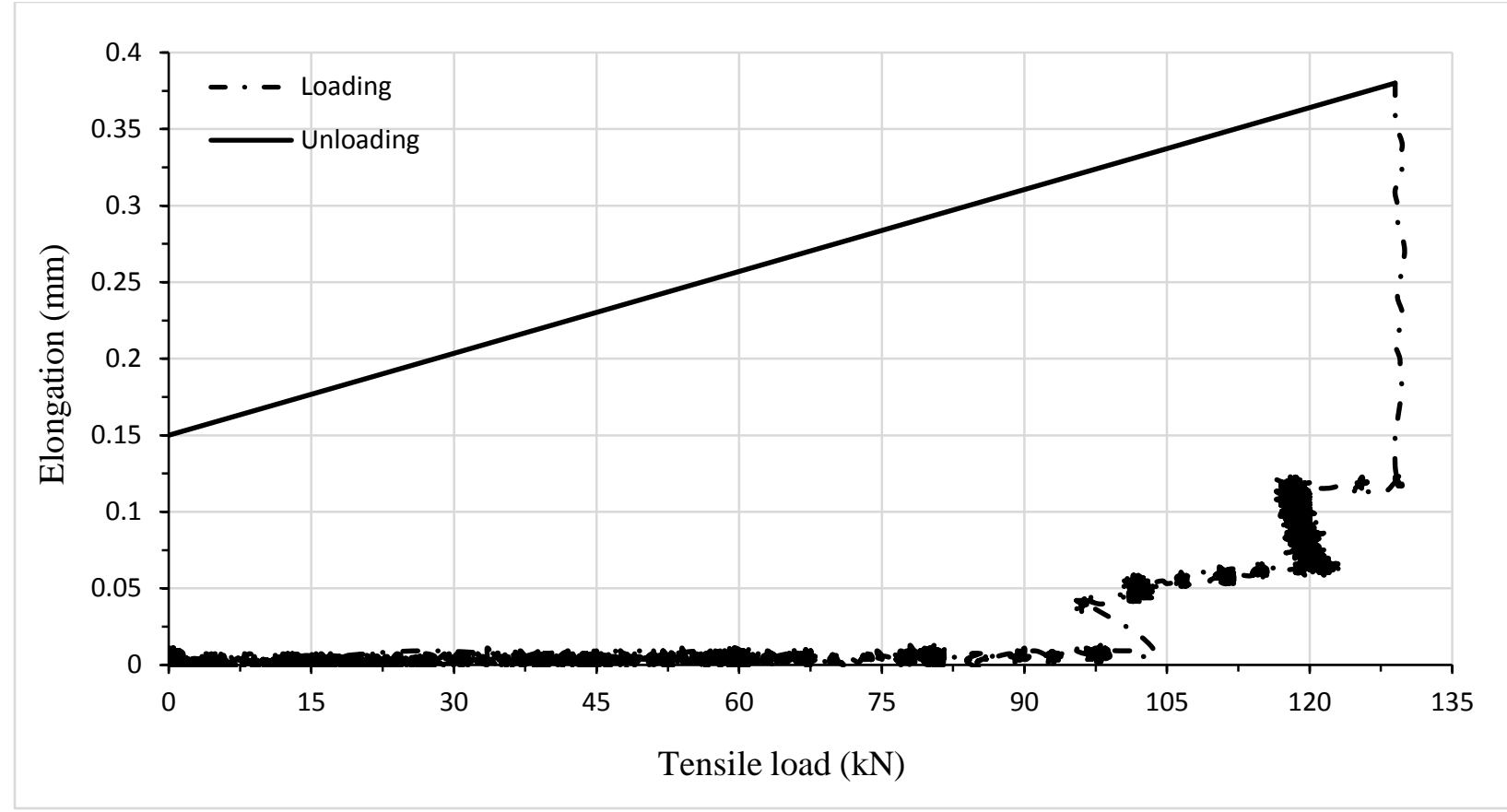

(d) NC4

Figure 4-5: Load- relative horizontal displacement at mid-span of a) NC1 b) NC2 c) NC3 d) NC4 (before repair)

\section{4-1-3 Leakage behavior of the NC panels}

Direct tensile loading was applied on the specimens until formation of a major crack (due to brittle nature of NC) at pre-determined location (mid-span), along with a high rate water leakage. Leakage rate was measured under different water pressures of 4, 8, 12, and 16 psi, equivalent to 27.6, 55.2, $82.74,110.32 \mathrm{kPa}$ and $2.82,5.64,8.45$, and $11.26 \mathrm{~m}$ height of water, respectively. The water pressure was set to 4 psi at the beginning of the test. Increasing the direct tensile load and formation of a major at the middle of the sample resulted in a high rate of leakage. Table 4-2 and Figure 4-6 present the leakage rate of the NC panels under various water pressures. Leakage rate of the specimens varied from 3996 to $4870 \mathrm{ml} / \mathrm{hr}, 8103$ to $10614 \mathrm{ml} / \mathrm{hr}, 10389$ to $13872 \mathrm{ml} / \mathrm{hr}$, and 12710 to $17790 \mathrm{ml} / \mathrm{hr}$ under water pressures of $4,8,12$, and $16 \mathrm{Psi}$, respectively. There is an intimate relationship between crack width and leakage rate. To illustrate, NC2 showing the lowest cracking load and crack width experienced the lowest leakage rate under different water pressures. 
However, NC4 having the highest cracking load and crack width exhibited the highest leakage rate. It can be observed in Figure 4-6 that increasing the water pressure resulted in higher leakage in all NC samples. Leakage rate of the NC1 and NC2 enhanced from $4218 \mathrm{ml} / \mathrm{hr}$ to $14935 \mathrm{ml} / \mathrm{hr}$ and $3996 \mathrm{ml} / \mathrm{hr}$ to $12710 \mathrm{ml} / \mathrm{hr}$, respectively, by increasing the water pressure from $4 \mathrm{psi}$ to $16 \mathrm{psi}$. Furthermore, increasing the water pressure from 4 psi to 16 psi enhanced the leakage rate of NC3 and NC4 from $4589 \mathrm{ml} / \mathrm{hr}$ to $15295 \mathrm{ml} / \mathrm{hr}$ and $4870 \mathrm{ml} / \mathrm{hr}$ to $17790 \mathrm{ml} / \mathrm{hr}$, respectively. Figure 47 illustrates the high rate of leakage in NC samples before the repair.

Table 4-2: Leakage rate ( $\mathrm{ml} / \mathrm{hr}$ ) of the $\mathrm{NC}$ samples under different water pressures (before repair)

\begin{tabular}{|c|c|c|c|c|}
\hline $\begin{array}{c}\text { Water pressure } \\
\text { (psi) }\end{array}$ & NC1 & NC2 & NC3 & NC4 \\
\hline 4 & 4218 & 3996 & 4589 & 4870 \\
\hline 8 & 9210 & 8103 & 9262 & 10614 \\
\hline 12 & 12278 & 10389 & 11556 & 13872 \\
\hline 16 & 14935 & 12710 & 15295 & 17790 \\
\hline
\end{tabular}

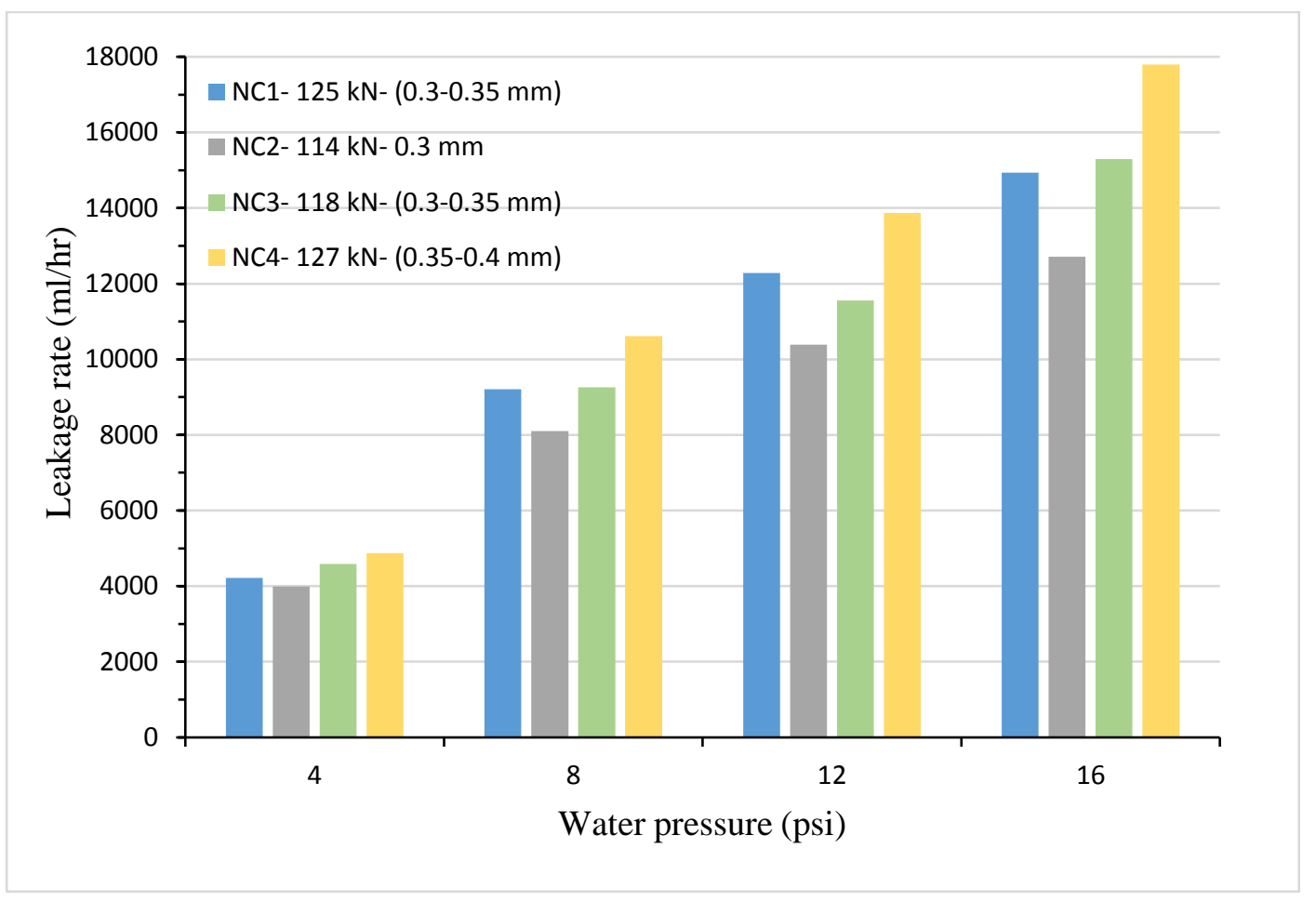

Figure 4-6: Leakage rate of the NC panels under different water pressures 


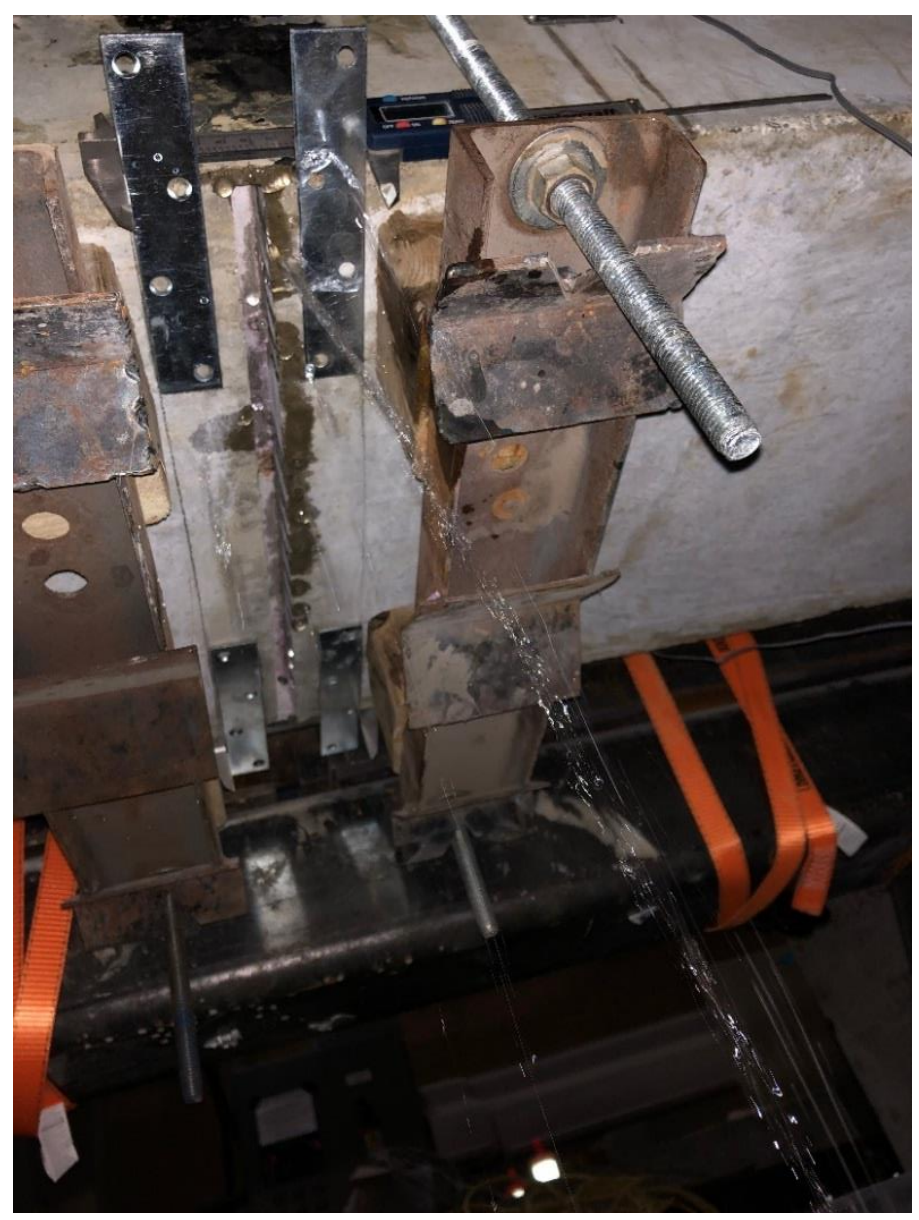

Figure 4-7: Extensive leakage in NC specimens at crack initiation (before repair)

Table 4-3 summarizes the load at leakage initiation, crack width, and leakage rate of the NC panels. It can be observed from the Table that NC2 exhibited the lowest crack width along with the lowest leakage rate under different water pressures. On the other hand, NC4 showed the highest crack and leakage rate under various water pressures. NC4 experienced higher leakage rates in the order of 1.22 to 1.4 times greater than NC2 under different water pressures. Furthermore, increasing the water pressure enhanced the rate of water leakage in NC panels. To illustrate, enhancement of water pressure from 4 psi to 16 psi resulted in increasing the leakage rate of water by a factor of 3.18 and 3.65 in NC2 and NC4, respectively. This can be explained by lower crack width of NC2 compared to NC4. 
Table 4-3: Summary of the load, crack width, and leakage rate in NC panels (before repair)

\begin{tabular}{|c|c|c|c|c|}
\hline Specimen & $\begin{array}{l}\text { Tensile load } \\
(\mathrm{kN})\end{array}$ & $\begin{array}{c}\text { Crack width } \\
(\mathrm{mm})\end{array}$ & $\begin{array}{c}\text { Water pressure } \\
(\mathrm{psi})\end{array}$ & $\begin{array}{c}\text { Leakage rate } \\
(\mathrm{ml} / \mathrm{hr})\end{array}$ \\
\hline \multirow{4}{*}{$\mathrm{NC} 1$} & \multirow{4}{*}{125} & \multirow{4}{*}{$0.3-0.35$} & 4 & 4218 \\
\hline & & & 8 & 9210 \\
\hline & & & 12 & 12278 \\
\hline & & & 16 & 14935 \\
\hline \multirow{4}{*}{$\mathrm{NC} 2$} & \multirow{4}{*}{114} & \multirow{4}{*}{0.3} & 4 & 3996 \\
\hline & & & 8 & 8103 \\
\hline & & & 12 & 10389 \\
\hline & & & 16 & 12710 \\
\hline \multirow{4}{*}{ NC3 } & \multirow{4}{*}{118} & \multirow{4}{*}{$0.3-0.35$} & 4 & 4589 \\
\hline & & & 8 & 9262 \\
\hline & & & 12 & 11556 \\
\hline & & & 16 & 15295 \\
\hline \multirow{4}{*}{$\mathrm{NC} 4$} & \multirow{4}{*}{127} & \multirow{4}{*}{$0.35-0.4$} & 4 & 4870 \\
\hline & & & 8 & 10614 \\
\hline & & & 12 & 13872 \\
\hline & & & 16 & 17790 \\
\hline
\end{tabular}

\section{4-1-4 Self-healing of the NC panels}

Self-healing ability of the NC panels was investigated by monitoring the leakage rate of the NC2 under water pressure of $7 \mathrm{psi}$ ( $48.27 \mathrm{kPa}$ or $4.93 \mathrm{~m}$ height of water) and sustained monotonic tensile loading of $114 \mathrm{kN}$. For this purpose, after measuring the leakage rate of the samples under different water pressure, the water pressure was fixed at $7 \mathrm{psi}$ and the leakage rate was recorded to attain a constant leakage rate or $100 \%$ self-healing of the tension-induced cracks. As the time prolonged, tension induced crack started to heal and resulted in reducing the leakage rate. Short-term selfhealing of the NC2 specimen during the first 35 hours is shown in Figure 4-8. The leakage rate 
decreased from $7867 \mathrm{ml} / \mathrm{hr}$ to $5035 \mathrm{ml} / \mathrm{hr}$ by $36 \%$ during the first 35 hours of leakage monitoring. NC2 sample exhibited a significant reduction of $19 \%$ in the leakage rate during the first 8 hours. It means that NC2 experienced almost $50 \%$ of the short-term self-healing (35 hours) during the first 8 hours. This can be explained by the fact that there are more available un-reacted cementitious materials during the first few hours to hydrate and block the water pathway through the crack by self-healing products. Therefore, self-healing rate decays over time.

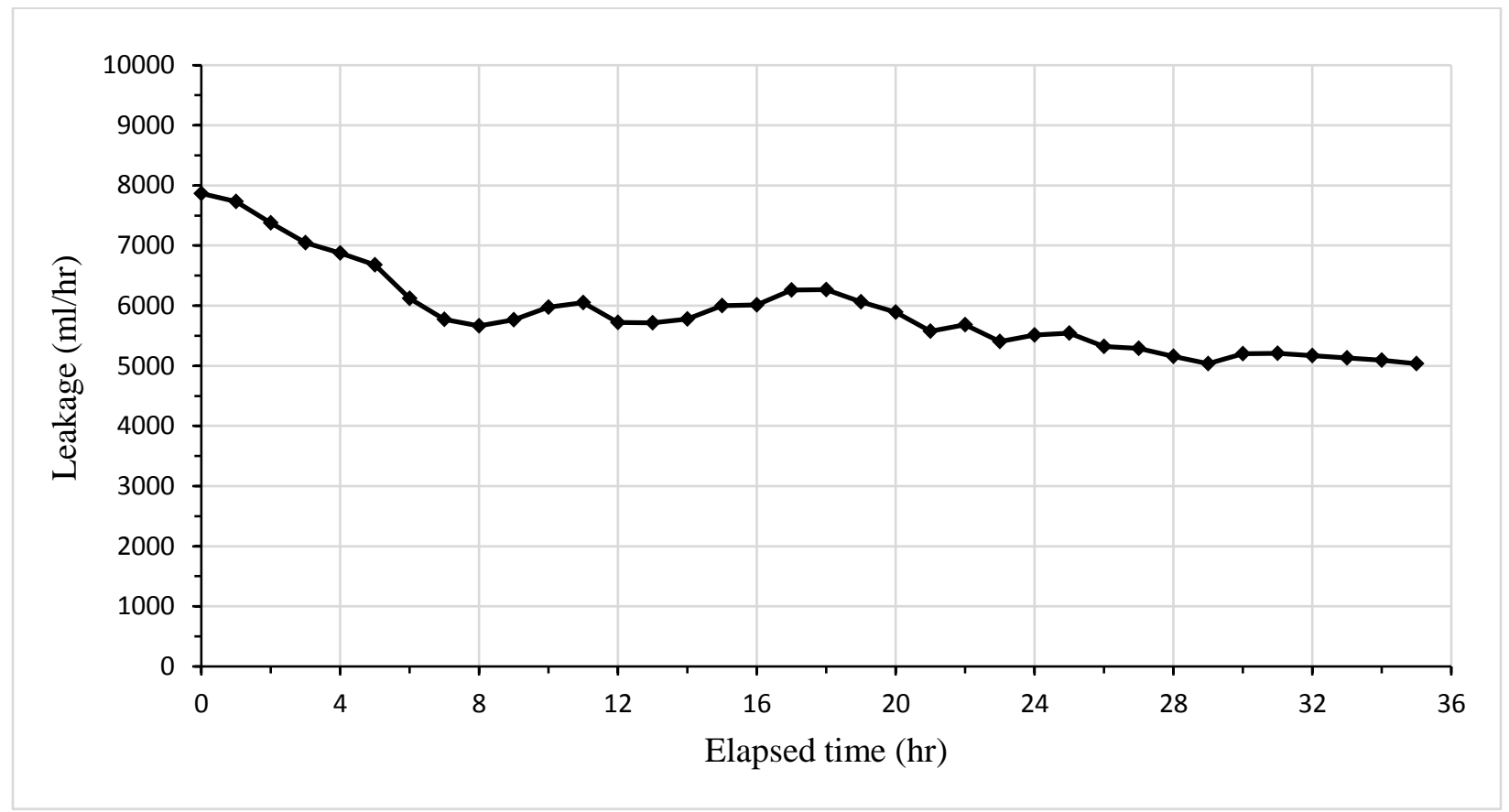

Figure 4-8: Short-term self-healing of NC2 sample (before repair)

Long-term self-healing of the NC2 specimen is illustrated in Figure 4-9. No significant change in the leakage rate was observed after 35 hours of monitoring the leakage. Therefore, recording the leakage rate was stopped after 4 days (95 hours). Leakage rate of the NC2 sample decreased from $7867 \mathrm{ml} / \mathrm{hr}$ to $4983 \mathrm{ml} / \mathrm{hr}$ by $37 \%$ after 4 days. It means that there was no significant change in the leakage rate of the NC2 sample after 35 hours up to 95 hours. NC2 sample achieved a total self- 
healing of $35 \%$, which occurred mostly during the first 35 hours. It is also interesting to note that $19 \%$ out of this $35 \%$ happened during the first 8 hours.

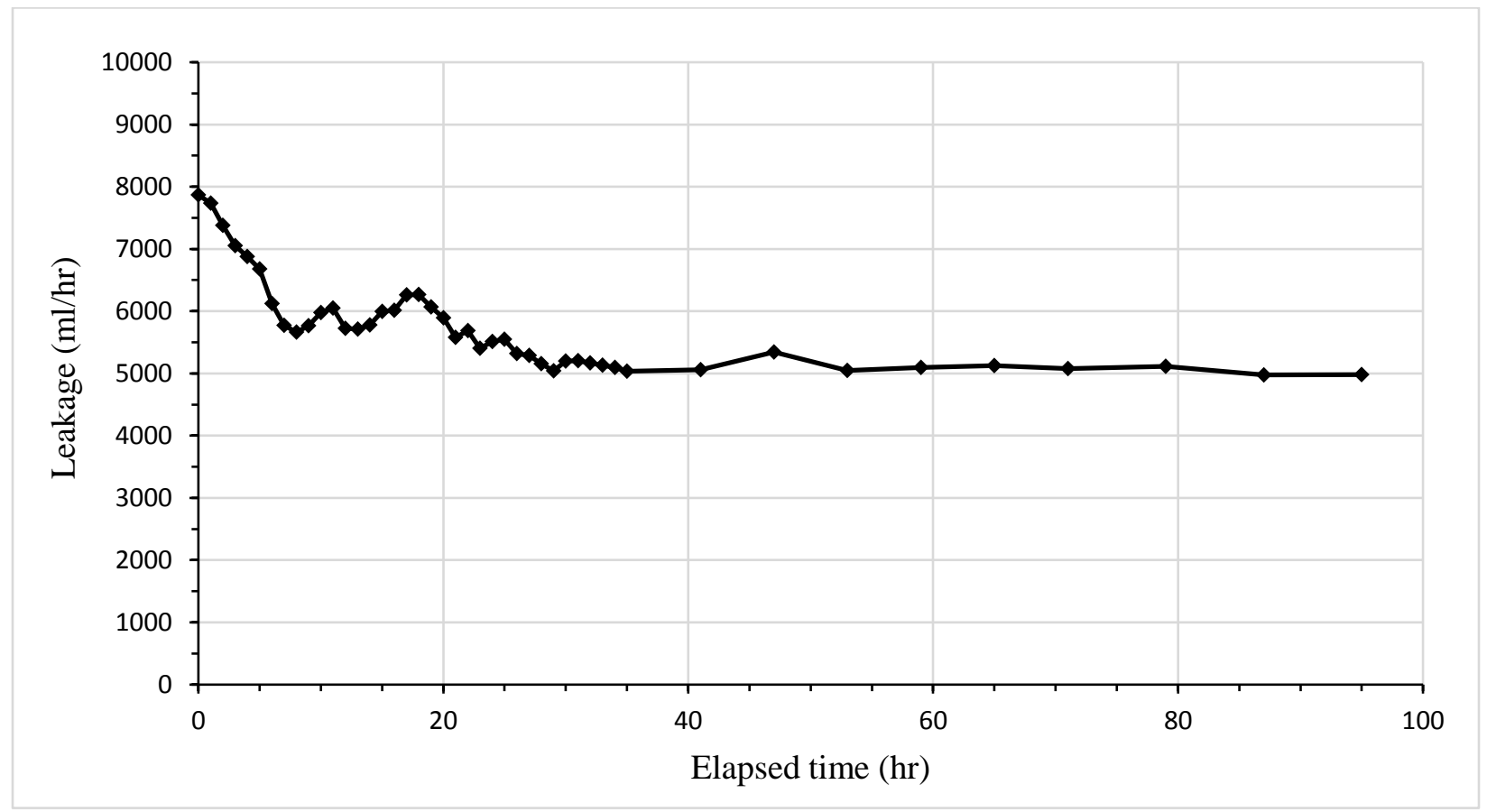

Figure 4-9: Long-term self-healing of NC2 sample (before repair)

\section{4-2 Performance of the repaired $\mathrm{RC}$ panels}

Tension induced cracks in $\mathrm{RC}$ panels ( $\mathrm{NC} 1, \mathrm{NC} 2, \mathrm{NC} 3$, and $\mathrm{NC} 4)$ were repaired using ECC slag, ECC fly ash, high-strength epoxy, and GFRP laminates. The behavior of repaired RC panels under monotonic tensile loading is presented in this section. The performance of RC panels repaired with each material was compared with respect to the NC panels before applying the repair.

\section{4-2-1 Performance of ECC slag repaired RC panel}

Monotonic tensile loading was applied on the NC panel repaired with a $50 \mathrm{~mm}$ layer of ECC slag with the same procedure as outlined in Chapter 3. Direct tensile loading applied to the specimen through the hydraulic jacks with increments of $10 \mathrm{kN}$. The load kept increasing until leakage 
initiation observed at the notch location. The first micro-cracks started to occur in ECC repair layer at midspan (where the crack existed in NC panel) at the load of $112 \mathrm{kN}$. However, no significant leakage was observed at this level of loading. Increasing the monotonic tensile loading resulted in a relatively low rate of leakage at $139 \mathrm{kN}$ at mid-span. Table 4-4 summarizes the tensile load and crack width in NC panel and ECC repair layer at each stage of loading. The residual crack width in NC panel before the repair was $0.11 \mathrm{~mm}$ and was increased by applying the tensile loading on the repaired sample. At loading level of $112 \mathrm{kN}$, at which ECC slag repair layer started to develop micro-cracks with average crack width of 50 Microns, crack width in the NC panel reached 0.36 $\mathrm{mm}$. The load at which leakage initiated, which is an important parameter in this study, was equal to $139 \mathrm{kN}$ along with a crack width of $0.15 \mathrm{~mm}$ in the ECC slag repair layer and $0.42 \mathrm{~mm}$ in the NC panel.

Table 4-4: Tensile load and crack width in NC1 panel and ECC repair layer

\begin{tabular}{|c|c|c|}
\hline \multirow{2}{*}{ Tensile load $(\mathrm{kN})$} & \multicolumn{2}{|c|}{ Crack width $(\mathrm{mm})$} \\
\cline { 2 - 3 } & ECC slag repair layer & NC panel \\
\hline 0 & 0 & 0.11 \\
\hline 20 & 0 & 0.15 \\
\hline 40 & 0 & 0.19 \\
\hline 60 & 0 & 0.23 \\
\hline 80 & 0 & 0.28 \\
\hline 100 & 0 & 0.33 \\
\hline 112 & 0.05 & 0.36 \\
\hline 120 & 0.10 & 0.38 \\
\hline 139 & 0.15 & 0.42 \\
\hline
\end{tabular}

At this level of loading, the leakage rate was measured under different water pressures. Figure 410 presents the leakage rate in $\mathrm{NC} 1$ panel repaired with ECC slag at the loading stage of $139 \mathrm{kN}$ under various water pressures of $4,8,12$, and $16 \mathrm{psi}$ and compares to the leakage rate under the 
same water pressures in $\mathrm{NC} 1$ before repair at the loading stage of $125 \mathrm{kN}$. It can be observed that the leakage rate was significantly decreased after repairing the NC1 specimen with ECC slag even at a higher level of loading. To illustrate, leakage rate of the NC1 sample was recorded as 4218, 9210,12278 , and $14935 \mathrm{ml} / \mathrm{hr}$ before the repair under water pressures of $4,8,12$, and $16 \mathrm{psi}$, respectively, while the leakage rate considerably reduced to the values of $166,368,446$, and 517 $\mathrm{ml} / \mathrm{hr}$ under $4,8,12$, and 16 psi water pressures in $\mathrm{NC} 1$ repaired with ECC slag. Considerably lower leakage rate in $\mathrm{NC} 1$ repaired with $\mathrm{ECC}$ slag compared to $\mathrm{NC} 1$ before repair can be attributed to the presence of micro-cracks in ECC layer. Therefore, water should penetrate through the full depth of micro-cracks in ECC layer, passing through the bond between the ECC layer and NC panel, and travel the depth of the NC panel to leak from the bottom face of the NC panel. This mechanism potentially reduced the leakage rate of the $\mathrm{NC1}$ panel repaired with $\mathrm{ECC}$ slag. Figure 4-11 shows the leakage rate of $\mathrm{NC} 1$ specimen repaired with ECC slag under water pressure of 8 psi.

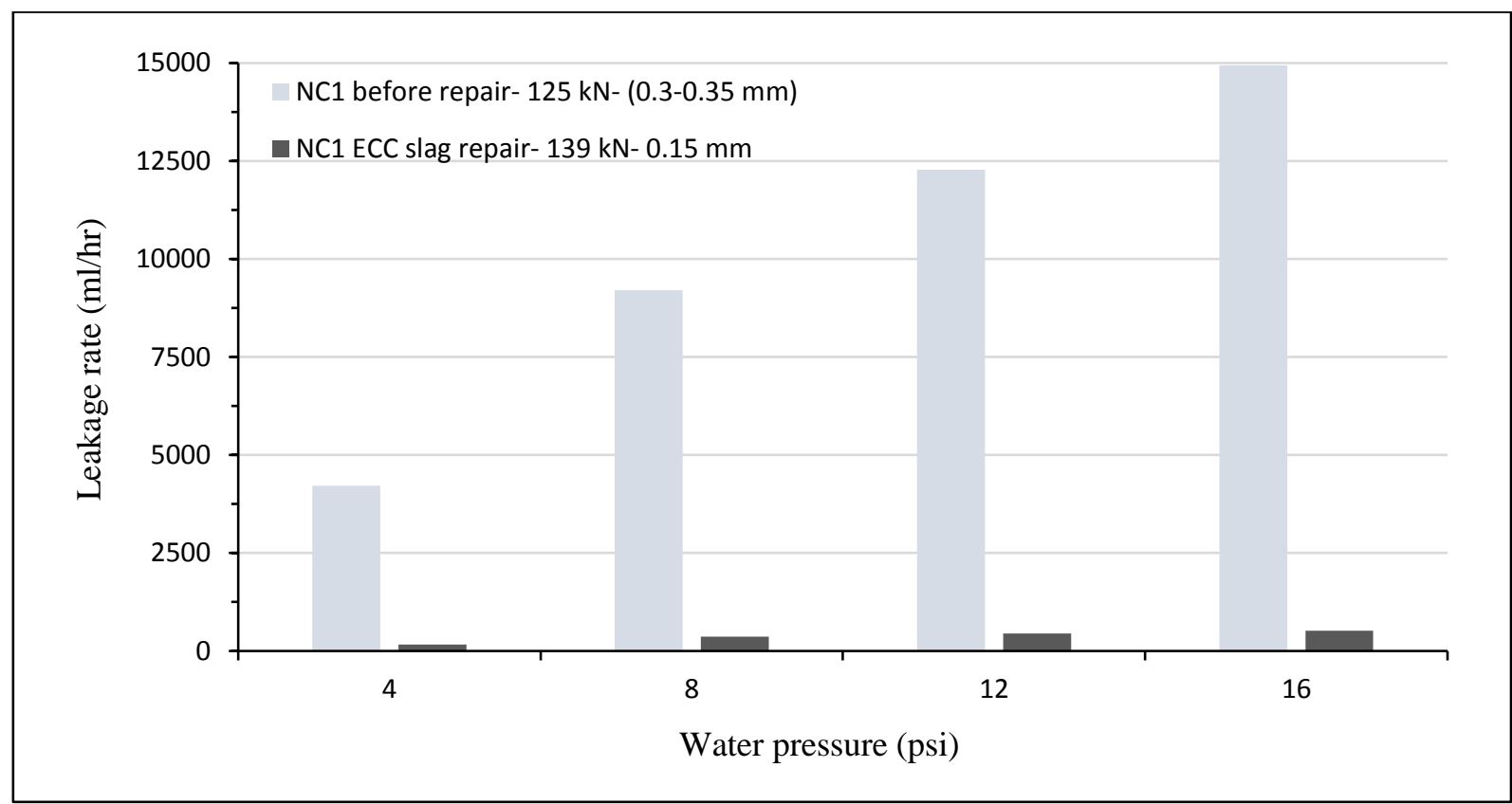

Figure 4-10: Comparison of the leakage behavior of NC before and after repair using ECC slag 


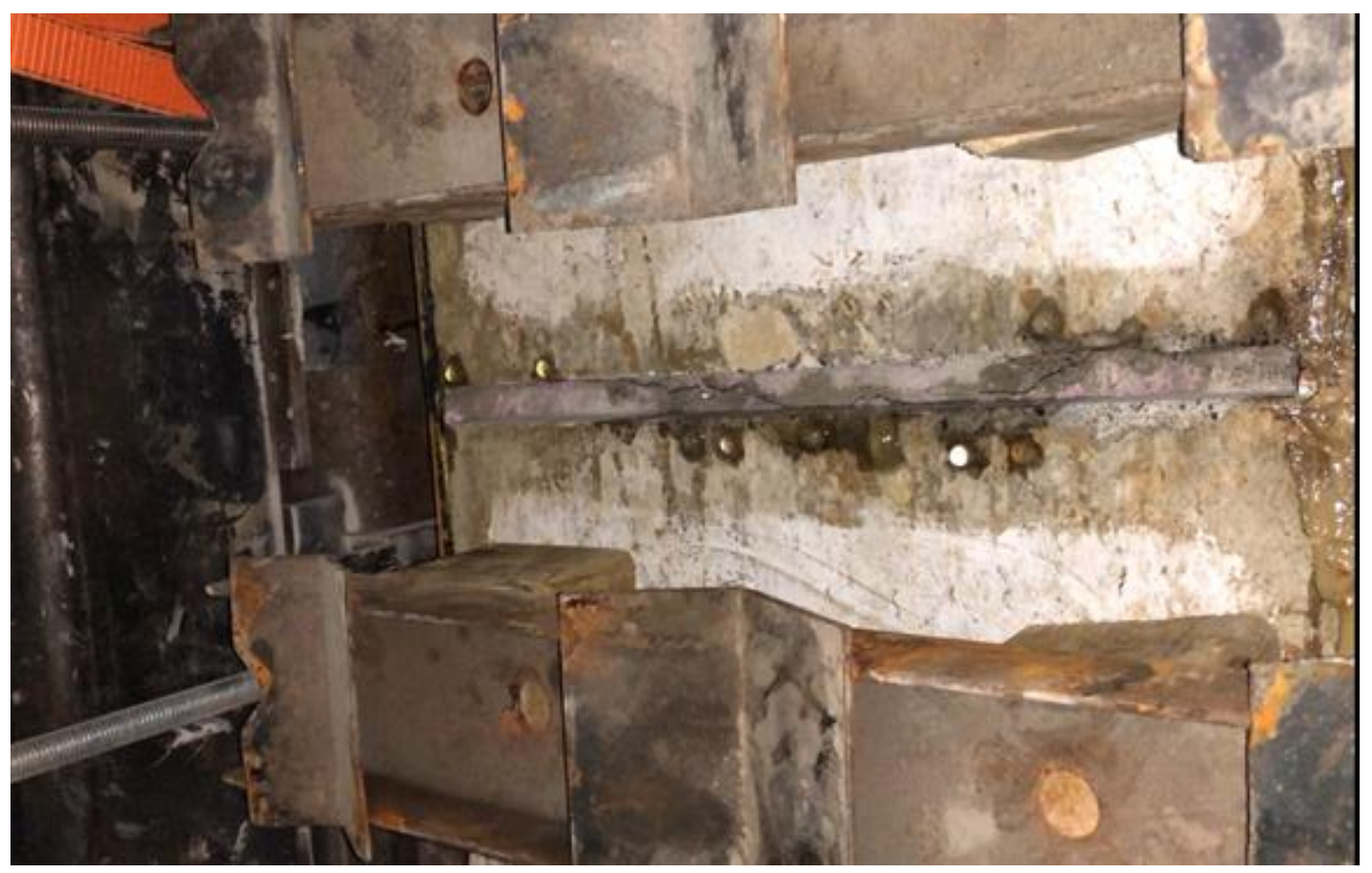

Figure 4-11: Leakage of the NC1 specimen repaired using ECC slag under 8 psi water pressure (bottom face)

Tensile steel strain development in NC1 repaired with ECC slag is shown in Figure 4-12. It is worth mentioning that steel strain shown in the Figure includes the residual strain from previous stage of loading (before repair). Tensile strain gradually increased in the rebar by applying the direct tensile loading on the specimen. At this level of loading, NC panel is cracked at midspan and has no contribution in carrying the tensile load, while ECC repair layer is uncracked and cooperates in carrying the tension along with the rebar. Therefore, the tensile load is transferred from the rebar embedded in the NC panel to the ECC repair layer through the bond between the rebar and NC panel and the bonding between the NC panel and ECC repair layer. At 1/6 span, where two steel strain gauges were placed, both NC panel and ECC repair layer are uncracked and active in tension. Tensile strain in the rebar increased more rapidly by the formation of microcracks in ECC repair layer at mid-span. 


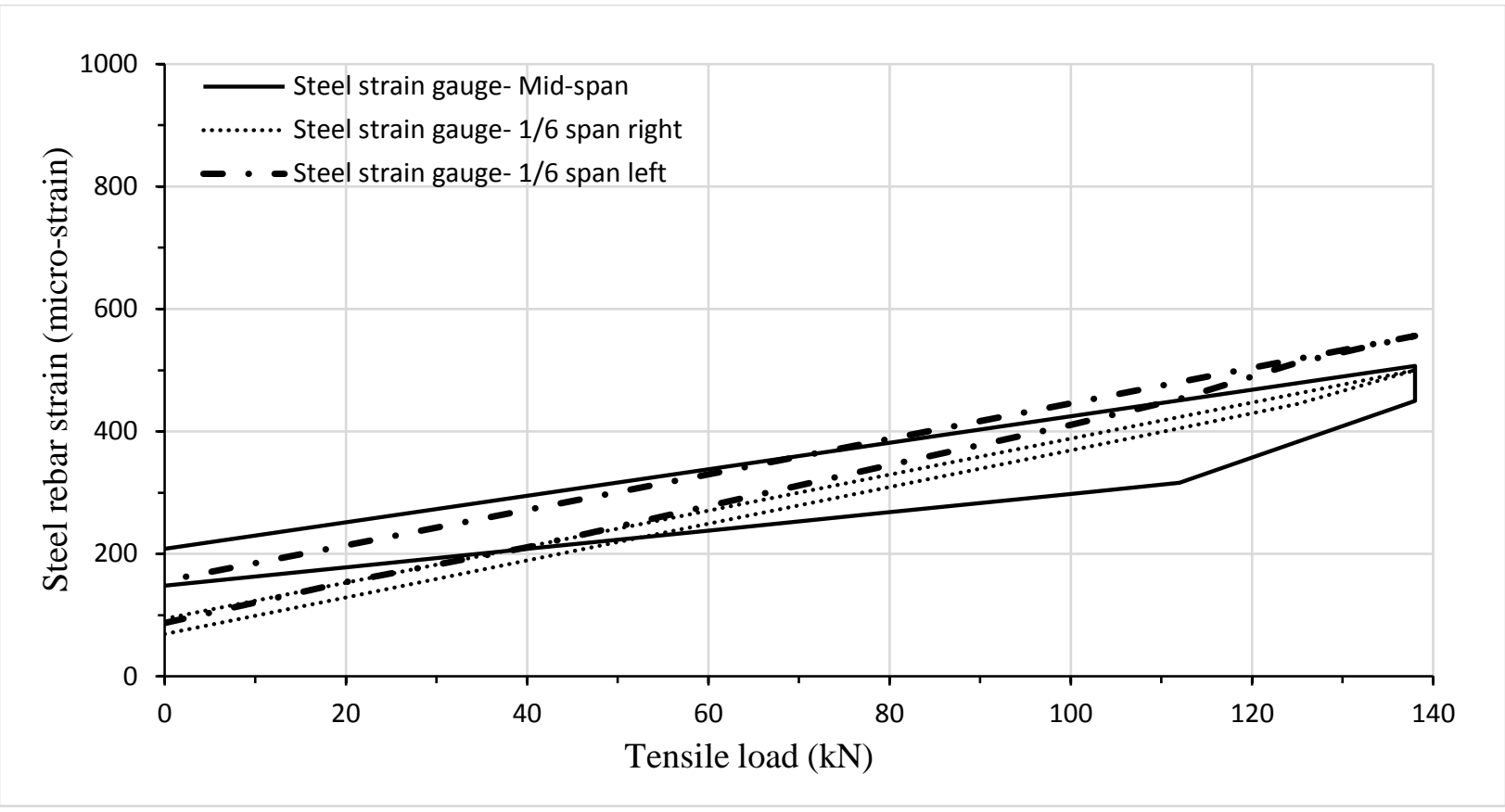

Figure 4-12: Tensile steel strain development in NC1 repaired using ECC slag

Figures 4-13 illustrates the load versus relative horizontal displacement of the repaired NC1 panel with respect to the mid-span (crack location). As the LVDTs were attached to the ECC repair layer, horizontal displacement started from zero excluding the residual crack width in NC1 panel from previous stage of loading. The horizontal displacement of ECC repair layer increased by formation of micro-cracks in ECC repair layer due to increasing the tensile loading. Gradual formation of micro-cracks in ECC layer with limited width of 0.05 to $0.15 \mathrm{~mm}$ resulted in horizontal displacement of $0.27 \mathrm{~mm}$ in ECC layer with respect to the mid-span. 


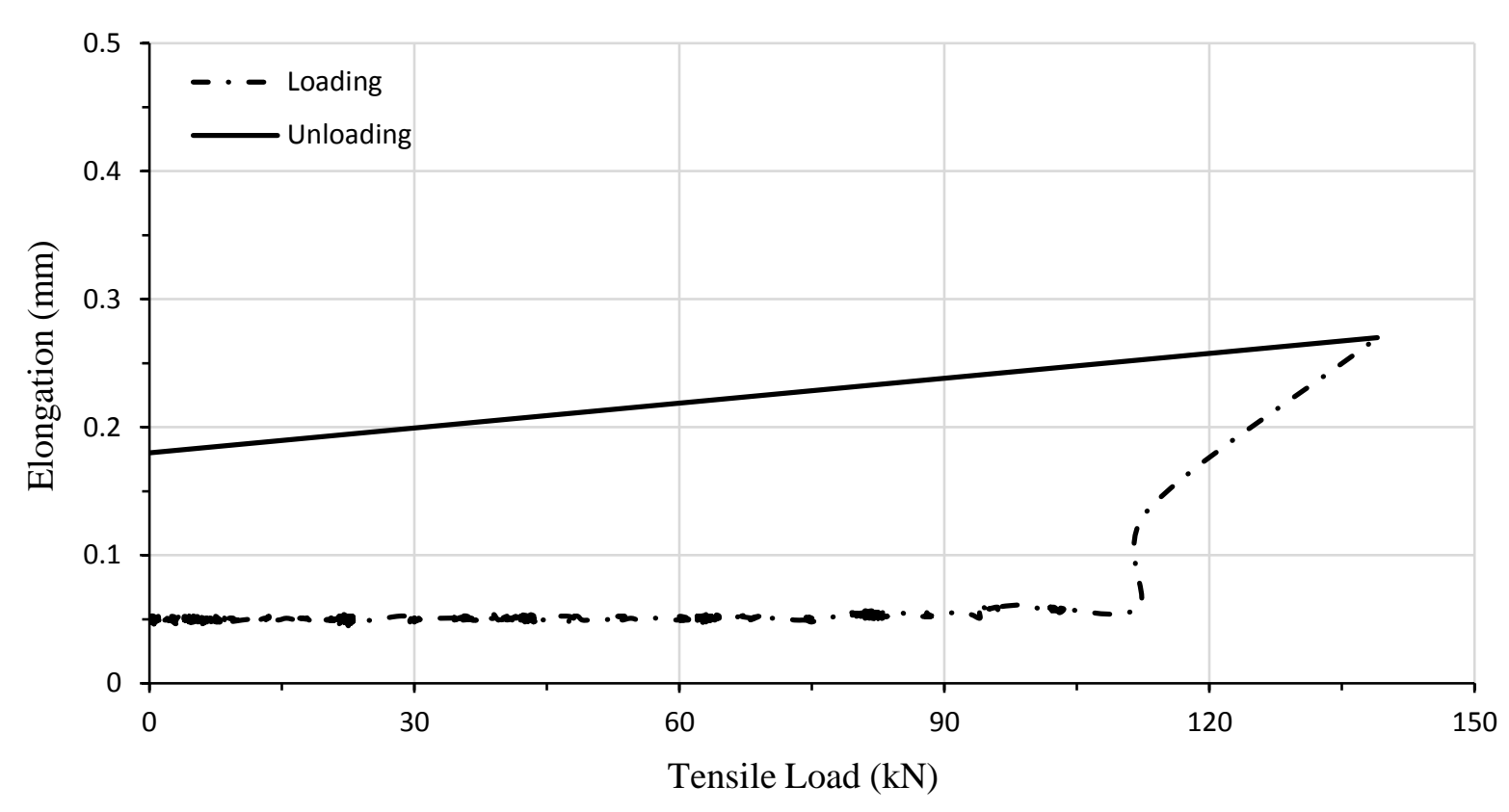

Figure 4-13: Tensile load versus relative horizontal displacement of the repaired NC1 panel with respect to the mid-span (crack location)

After measuring the leakage rate of the $\mathrm{NC1}$ repaired with ECC slag, water pressure in the chamber was set to 7 psi to investigate the self-healing behavior of the $50 \mathrm{~mm}$ ECC repair layer on top of the NC panel under a constant load of $139 \mathrm{kN}$ and fixed water pressure of 7 psi. For this purpose, leakage rate of the NC1 repaired with ECC slag was recorded continuously to achieve a constant leakage rate or to attain 100\% self-healing of the tension cracks. As the time prolonged, tension induced crack started to heal and resulted in reducing the leakage rate. In short-term investigation of the self-healing, the amount of water penetrated through the full depth of the tension cracks in ECC slag repair layer and NC panel and leaked from the bottom surface on the NC panel was measured over a one-hour time intervals. Short-term self-healing of the NC1 repaired with ECC slag during the first 35 hours is shown in Figure 4-14. The leakage rate reduced by $68 \%$ from 338 $\mathrm{ml} / \mathrm{hr}$ to $107 \mathrm{ml} / \mathrm{hr}$ during the first 35 hours. A sharp drop was observed in leakage rate during the first 10 hours in which the leakage rate decreased by $41 \%$ from $338 \mathrm{ml} / \mathrm{hr}$ to $199 \mathrm{ml} / \mathrm{hr}$. The rate of leakage reduction subsided as the time prolonged which is due to reduction in self-healing 
capacity of ECC repair layer and NC panel over time. This can be explained by the fact that there are less available cementitious materials over time to participate in self-healing of the concrete panels.

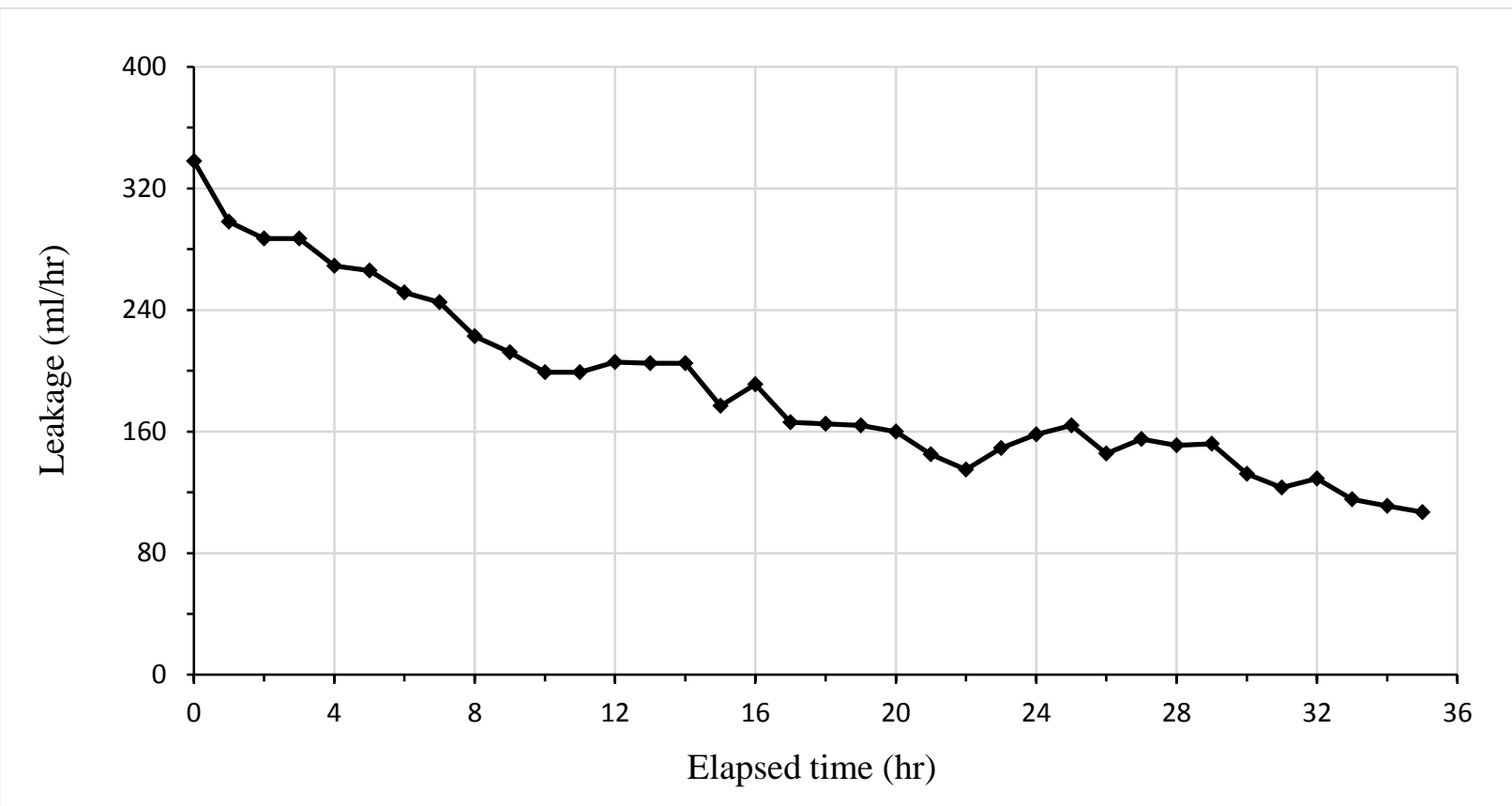

Figure 4-14: Short-term self-healing of the NC1 repaired using ECC slag

Figure 4-15 illustrates the long-term self-healing of the NC1 repaired with ECC slag over the period of 13 days. During the first 35 hours, leakage was recorded continuously each hour, while during the period of time between 35 hours up to 6 days (144 hours), leakage rate was measured for one hour in each 6 hours, followed by the last period of time from 6 days (144 hours) to 13 days (312 hours), leakage rate was recorded for one hour in each 8 hours. Leakage rate in the NC1 repaired with ECC slag reduced to zero after 13 days of monitoring the leakage rate which indicates the water flow with pressure of 7 psi was completely blocked by the self-healing products. Blockage of the water flow could occur either in the ECC repair layer or in the interface between ECC repair layer and NC panel. Achieving the zero-leakage rate is not necessarily an indication of $100 \%$ self-healing of the full depth crack in ECC repair layer and NC panel. It is worth- 
mentioning that obstruction created by self-healing products in partial depth of the section or in the interface of ECC repair layer and NC panel can prevent the leakage and make flow of water through the depth of the section impossible.

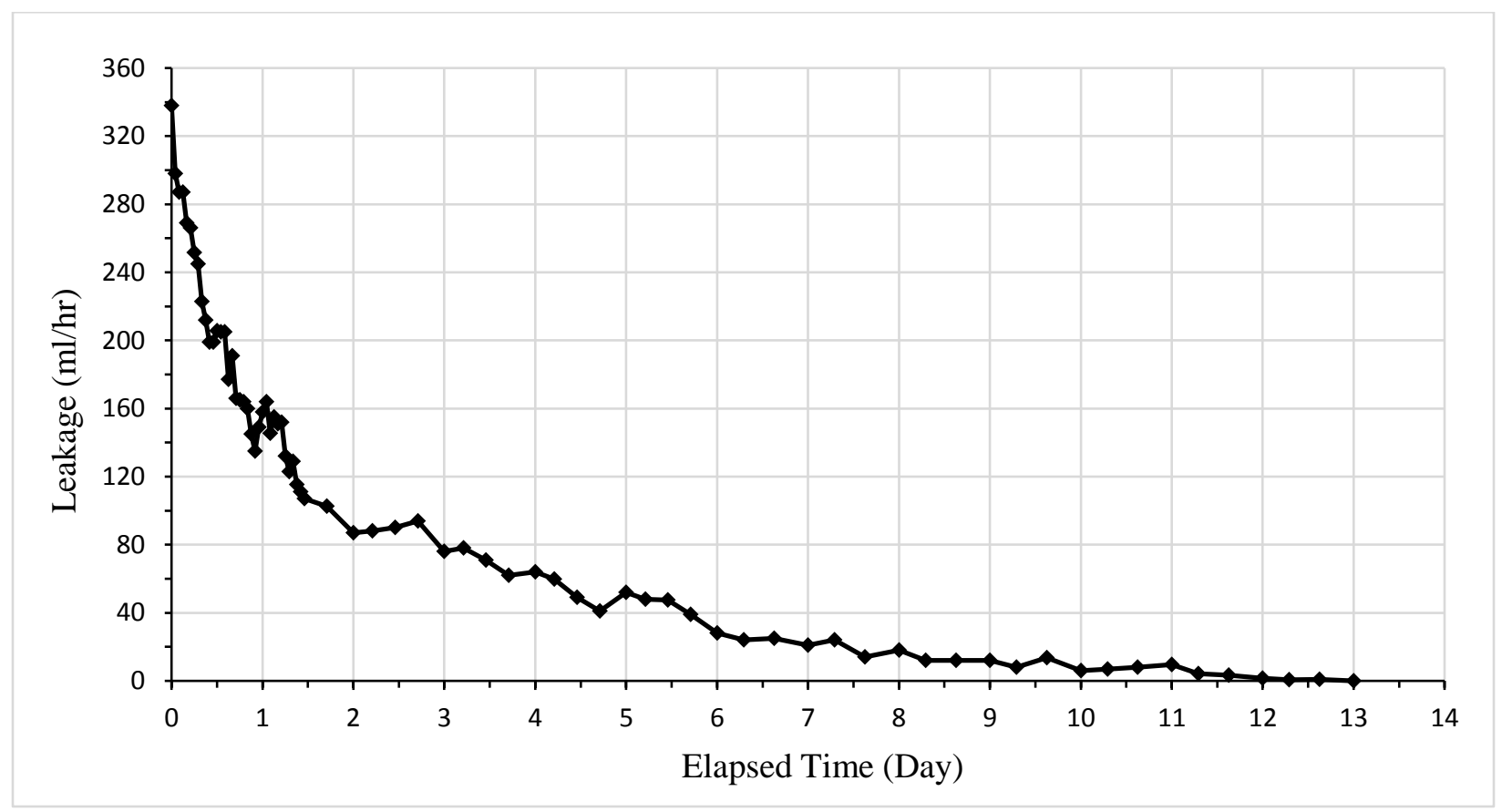

Figure 4-15: Long-term self-healing of the NC1 repaired using ECC slag

Table 4-5 summarizes the performance of the NC1 repaired with ECC slag in terms of strength, crack width and leakage behavior and compares to the $\mathrm{NC1}$ performance before the repair. As the tensile loading was applied on the $\mathrm{NC} 1$ panel before the repair, a major crack was formed at predetermined location (mid-span) at load of $125 \mathrm{kN}$ along with a crack width of $0.3-0.35 \mathrm{~mm}$, which was immediately followed by high rate leakage. However, after repairing the NC panel with 50 $\mathrm{mm}$ layer of ECC slag, by applying the monotonic tensile loading, a low rate of leakage initiated at the load $139 \mathrm{kN}$ by developing micro-cracks in ECC slag repair layer with maximum crack width of $0.15 \mathrm{~mm}$. The results show that $50 \mathrm{~mm}$ ECC slag repair layer improved strength, crack development, and leakage behavior of the NC panel. This can be explained by multiple microcracking behavior and tensile strain hardening behavior of ECC which resulted in increasing the 
load at leakage initiation, reducing the crack width, and consequently much lower leakage rate. Moreover, ECC slag repair layer retained the structural strength of the cracked NC panel. This can be attributed to the multiple micro-cracking behavior of ECC along with strain hardening and high tensile strain capacity, which shows the effectiveness of ECC in repairing RC structures. It is worth-mentioning that use of slag as the supplementary cementitious material (SCM) resulted in higher strength as well as larger crack width in ECC repair layer in comparison with ECC fly ash. Figure 4-16 compares the difference between leakage rate of the $\mathrm{NC}$ panel before repair and after repair with ECC slag.

Table 4-5: Comparison of the performance of $\mathrm{NC} 1$ before and after repair using ECC slag

\begin{tabular}{|c|c|c|c|c|}
\hline & $\begin{array}{c}\text { Tensile load } \\
(\mathrm{kN})\end{array}$ & $\begin{array}{l}\text { Mid-span crack } \\
\text { width }(\mathrm{mm})\end{array}$ & $\begin{array}{c}\text { Water } \\
\text { pressure (psi) }\end{array}$ & $\begin{array}{l}\text { Leakage rate } \\
\qquad(\mathrm{ml} / \mathrm{hr})\end{array}$ \\
\hline \multirow{4}{*}{ Before repair } & \multirow{4}{*}{125} & \multirow{4}{*}{$0.3-0.35$} & 4 & 4218 \\
\hline & & & 8 & 9210 \\
\hline & & & 12 & 12278 \\
\hline & & & 16 & 14935 \\
\hline \multirow{4}{*}{ ECC slag repair } & \multirow{4}{*}{139} & \multirow{4}{*}{0.15} & 4 & 166 \\
\hline & & & 8 & 369 \\
\hline & & & 12 & 447 \\
\hline & & & 16 & 518 \\
\hline
\end{tabular}




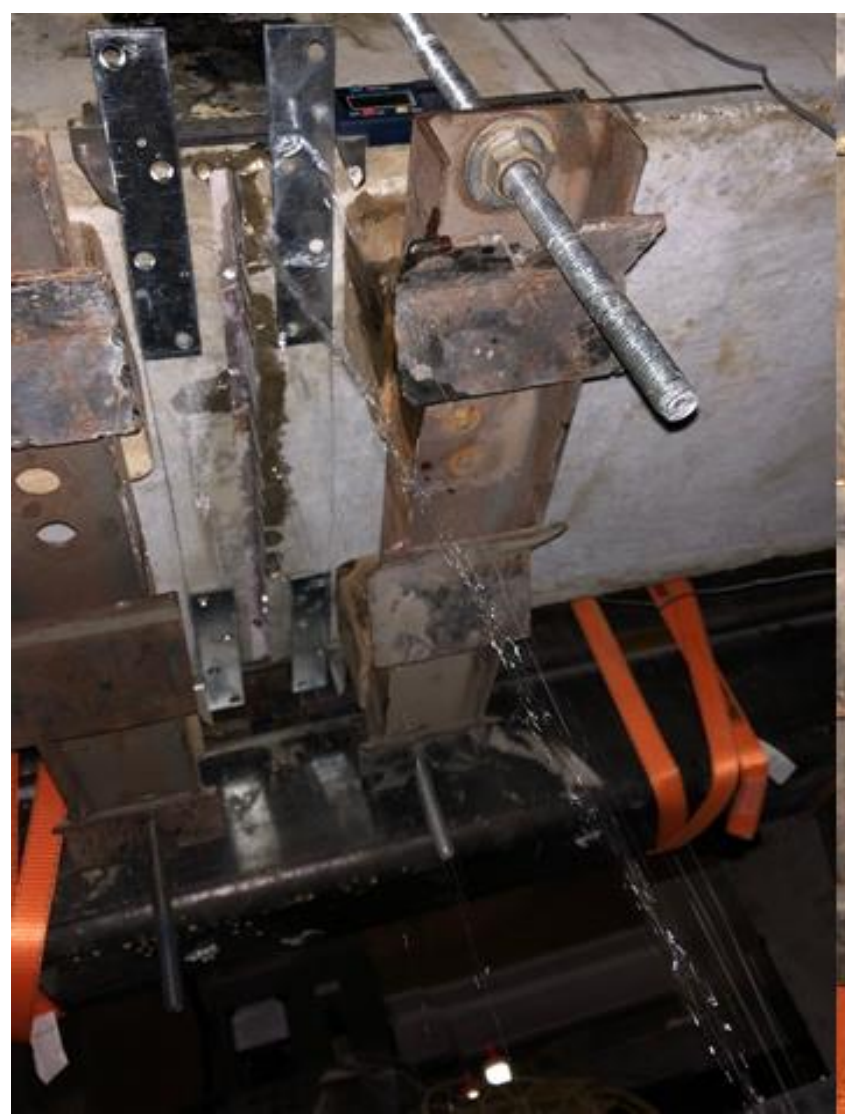

(a)

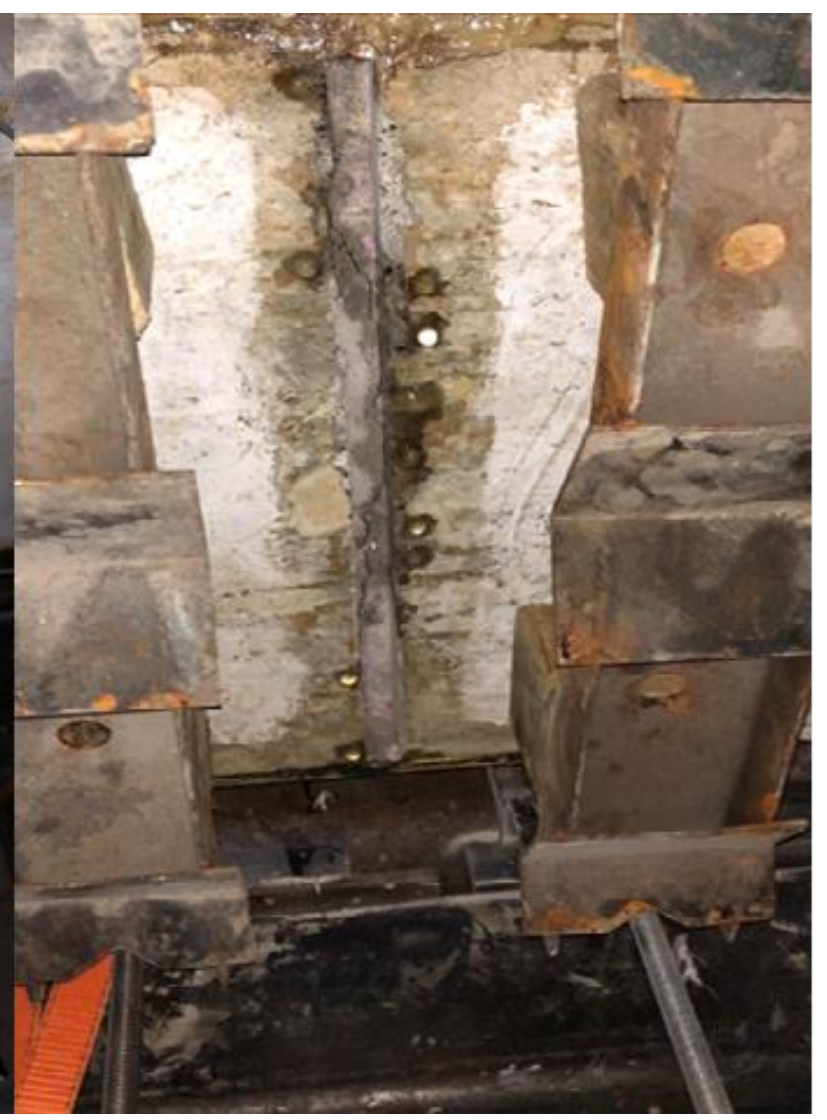

(b)

Figure 4-16: Leakage of the NC panel before and after repair using ECC slag a) Before repair b) After repair

\section{4-2-2 Performance of GFRP laminate repaired RC panel}

Direct tensile loading was applied on the NC panel repaired with two layers of GFRP laminates according to the procedure as outlined in Chapter 3. Direct tensile loading was applied to the specimen through the hydraulic jacks with increments of $10 \mathrm{kN}$. The load kept increasing until failure of the GFRP laminate and leakage initiation at the crack location, or reaching the maximum allowable tensile loading that can carried by the specimen due yielding of the rebar. The monotonic tensile load was applied to the specimen up to $400 \mathrm{kN}$, which is equivalent to the load at which yielding strain is developed in the rebar, and the test was stopped at this level of loading due to safety reasons. While the crack width in NC panel was increasing as a result of applying the tensile loading to the specimen, no failure was observed in GFRP laminate due to its high tensile capacity. 
Failure of the repaired specimen was governed by rebar yielding rather than GFRP laminate tensile rupture or debonding at the interface of GFRP and concrete substrate. Table 4-6 and Figure 4-17 present the average crack width at mid-span, 1/6-spans from the ends, and both end-spans of the NC2 specimen repaired with GFRP laminate at each stage of loading.

Table 4-6: Summary of the load and crack width of NC2 specimen after repair using GFRP

\begin{tabular}{|c|c|c|c|}
\hline \multirow{2}{*}{ Tensile load $(\mathrm{kN})$} & \multicolumn{3}{|c|}{ Crack width (mm) } \\
\hline & Mid-span & 1/6-spans & End-spans \\
\hline 0 & 0.14 & 0 & 0 \\
\hline 20 & 0.18 & 0 & 0 \\
\hline 40 & 0.23 & 0 & 0 \\
\hline 60 & 0.27 & 0 & 0 \\
\hline 80 & 0.32 & 0 & 0 \\
\hline 100 & 0.35 & 0 & 0 \\
\hline 120 & 0.37 & 0 & 0 \\
\hline 140 & 0.39 & 0 & 0 \\
\hline 160 & 0.41 & 0 & 0 \\
\hline 180 & 0.43 & 0 & 0 \\
\hline 204 & 0.45 & 0 & 0.15 \\
\hline 220 & 0.48 & 0 & 0.2 \\
\hline 240 & 0.51 & 0 & $0.2-0.25$ \\
\hline 260 & 0.54 & 0.1 & 0.3 \\
\hline 270 & 0.56 & $0.1-0.15$ & 0.33 \\
\hline 280 & 0.57 & 0.14 & $0.35-0.4$ \\
\hline 300 & 0.59 & $0.15-0.2$ & 0.4 \\
\hline 320 & 0.61 & 0.23 & 0.43 \\
\hline 340 & 0.66 & 0.25 & $0.45-0.5$ \\
\hline 360 & 0.71 & 0.28 & 0.5 \\
\hline 380 & 0.75 & 0.31 & 0.51 \\
\hline 400 & 0.78 & 0.33 & 0.53 \\
\hline
\end{tabular}

It can be observed that the mid-span crack width increased from the residual crack width of 0.14 $\mathrm{mm}$, at the beginning of loading, to $0.78 \mathrm{~mm}$ at $400 \mathrm{kN}$. At the loading stage of $204 \mathrm{kN}$, bond slip 
occurred between the rebar and concrete at both ends of the specimen simultaneously along with a crack as wide as $0.15 \mathrm{~mm}$ propagating toward top and bottom surfaces of the NC panel. At this stage of loading, no cracking was observed at 1/6-span and average crack width at mid-span was equal to $0.45 \mathrm{~mm}$. By increasing the tensile load, developed cracks at both ends propagated along the longitudinal direction of the specimen toward mid-span at bottom face. At loading stage of 260 $\mathrm{kN}$, bottom surface cracks propagated in transverse direction (extended to the entire width) causing cracks as wide as $0.1 \mathrm{~mm}$ at distance of $205 \mathrm{~mm}$ from the north end and $215 \mathrm{~mm}$ from the south end of the specimen (almost at 1/6 of the span from the ends). At this stage of loading crack widths at mid-span and end-span were $0.54 \mathrm{~mm}$ and $0.3 \mathrm{~mm}$, respectively. By further enhancing the direct tensile loading, cracks widths at mid-span, 1/6-span, and end-span increased gradually reaching $0.78 \mathrm{~mm}, 0.33 \mathrm{~mm}$, and $0.53 \mathrm{~mm}$ at $400 \mathrm{kN}$, respectively.

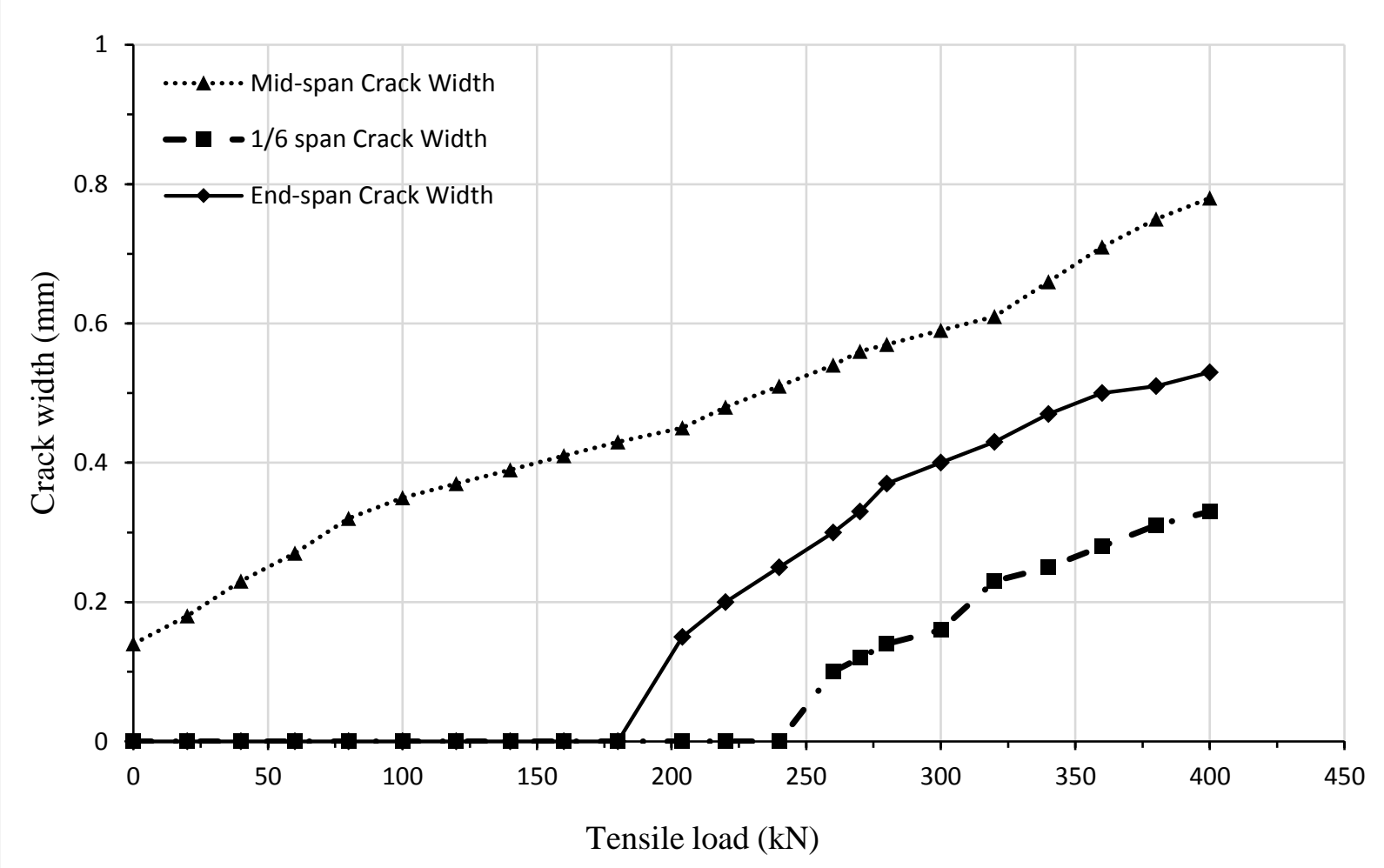

Figure 4-17: Tensile load vs. crack width of the NC2 specimen repaired using GFRP laminate 
For the purpose of investigating the effect of GFRP laminate on crack width development in the GFRP repaired specimen, a comparison is made between the crack width results in NC2 panel before and after repair using GFRP at various loading stages as shown in Figure 4-18. For this purpose, after measuring the leakage rate of the NC2 panel (before repair), it was re-loaded up to $114 \mathrm{kN}$ to obtain the mid-span crack growth data under direct tensile loading. A residual crack width of $0.14 \mathrm{~mm}$ was resulted from this stage of loading. It can be observed that in the range of tensile load lower than $110 \mathrm{kN}$, crack width developed at mid-span in GFRP repaired specimen is slightly greater compared to NC panel before the repair. This can be attributed to the residual crack width, residual tensile strain in the rebar, and non-recoverable bond-slip between the rebar and concrete resulted from stages of loading before repair. This is an indication of the fact that GFRP laminate has less restraining effect on crack width development at lower range of tensile loads due to initial bond slippage in the interface between the GFRP laminate and concrete surface. However, by increasing the tensile load up to $400 \mathrm{kN}$, GFRP laminate exhibited more significant restraining effect on mid-span crack width of the NC panel. 


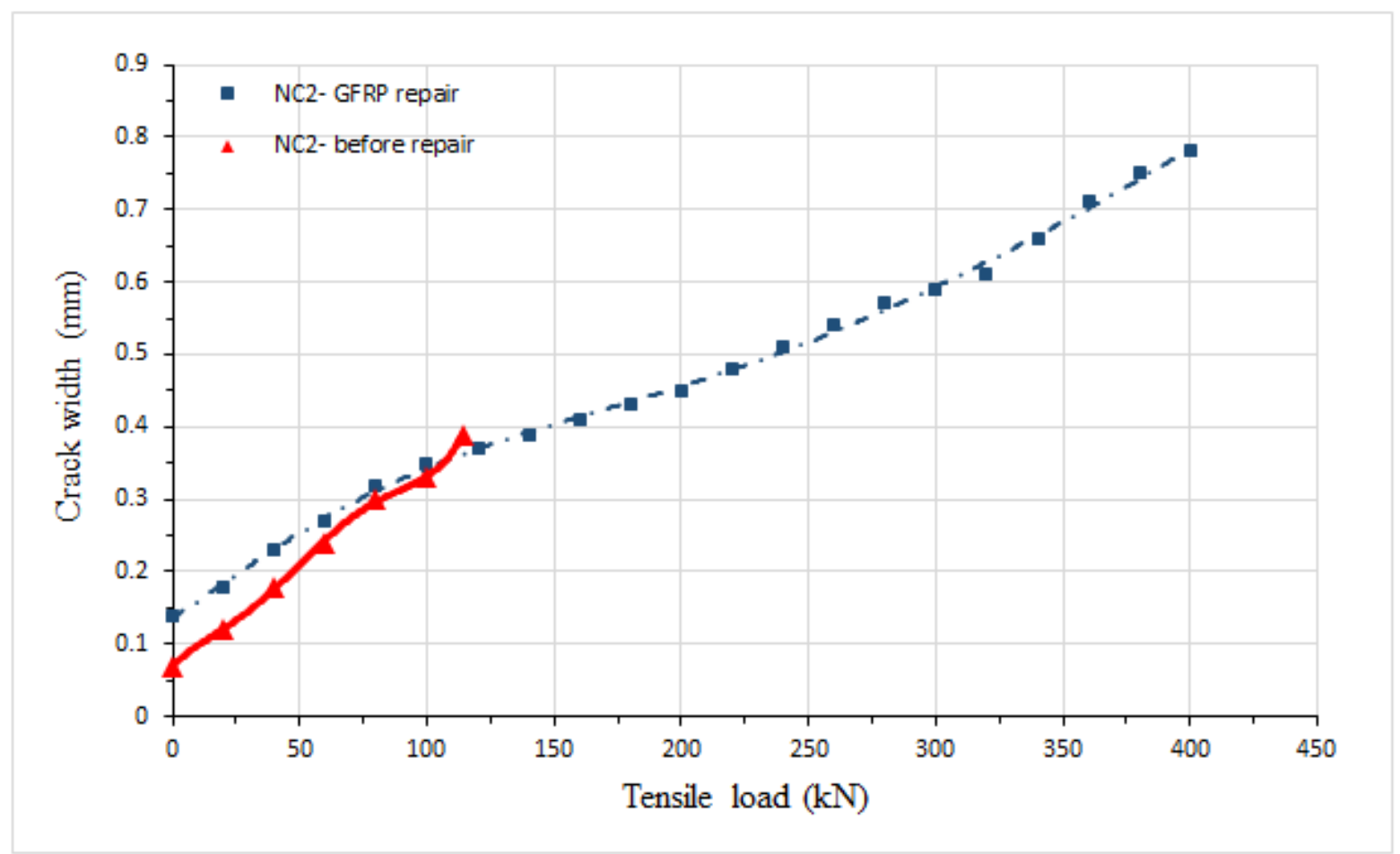

Figure 4-18: Mid-span crack width in NC2 panel before and after GFRP repair

Figure 4-19 presents tensile strain development in the rebar. Steel strain started from the residual values from the previous stage of loading. Tensile strain gradually increased in the rebar by applying the direct tensile loading on the specimen. At initial stages of loading, NC panel is cracked at mid-span and does not contribute in carrying the tensile load, while GFRP laminate is active in tension and cooperates in carrying the tensile load along with the rebar. Therefore, the tensile load is transferred from the rebar embedded in the NC panel to the GFRP laminate through the bond between the rebar and $\mathrm{NC}$ panel and bonding between the NC panel and GFRP laminate. However, at 1/6-span NC panel is uncracked and still capable of tolerating tensile load. By further applying the tensile load up to $400 \mathrm{kN}$, rebar tensile strain was increased. 


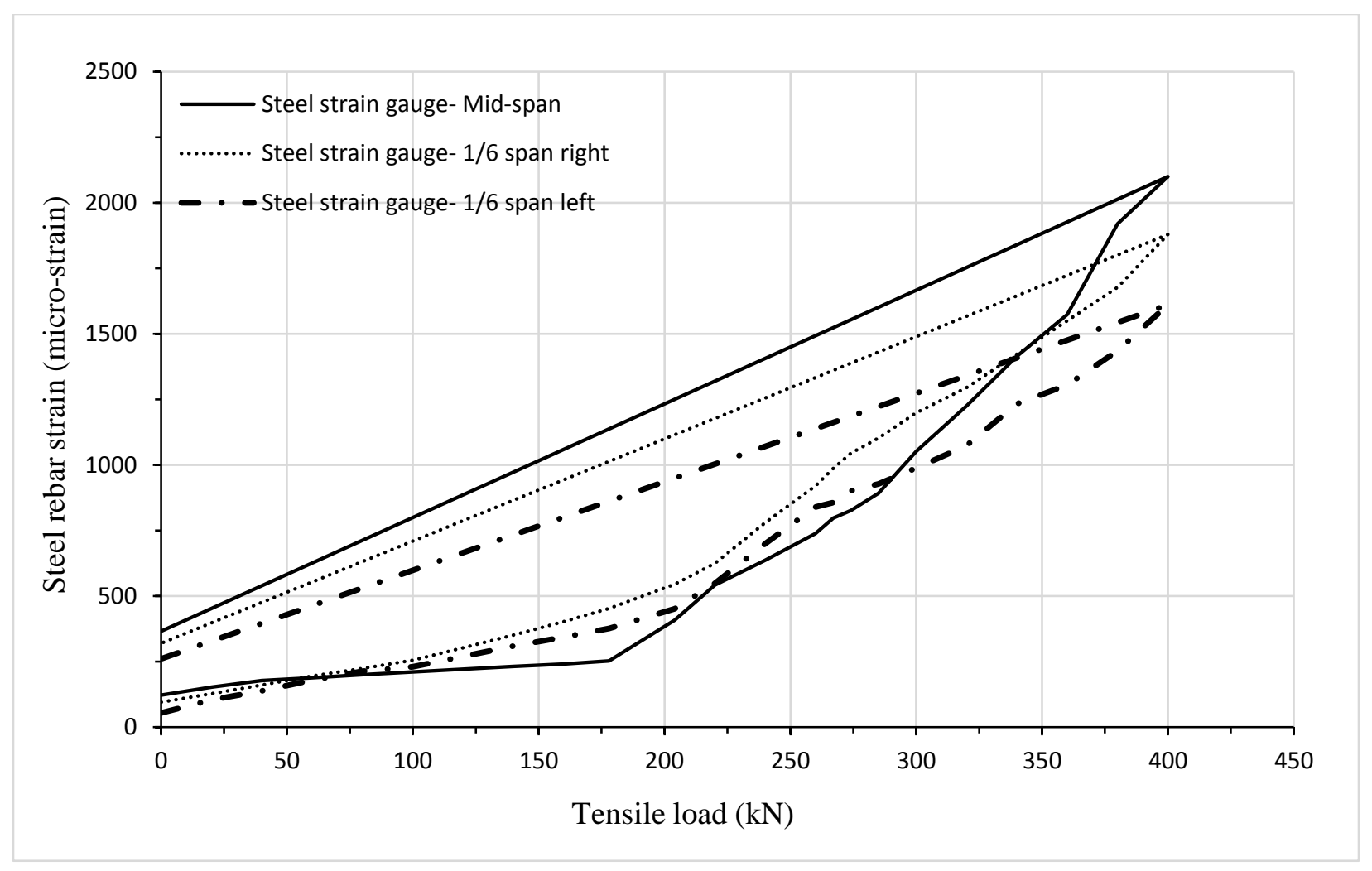

Figure 4-19: Tensile strain development in the rebar in GFRP repaired NC2 panel

Figures 4-20 illustrates the load versus relative horizontal displacement of the NC panel with respect to the mid-span (crack location). The horizontal displacement of the specimen starts from zero and does not include the residual crack from previous steps of loadings (before repair). The horizontal displacement of the NC panel increased by widening of the crack in NC panel at midspan due to incrementally increasing the tensile loading. 


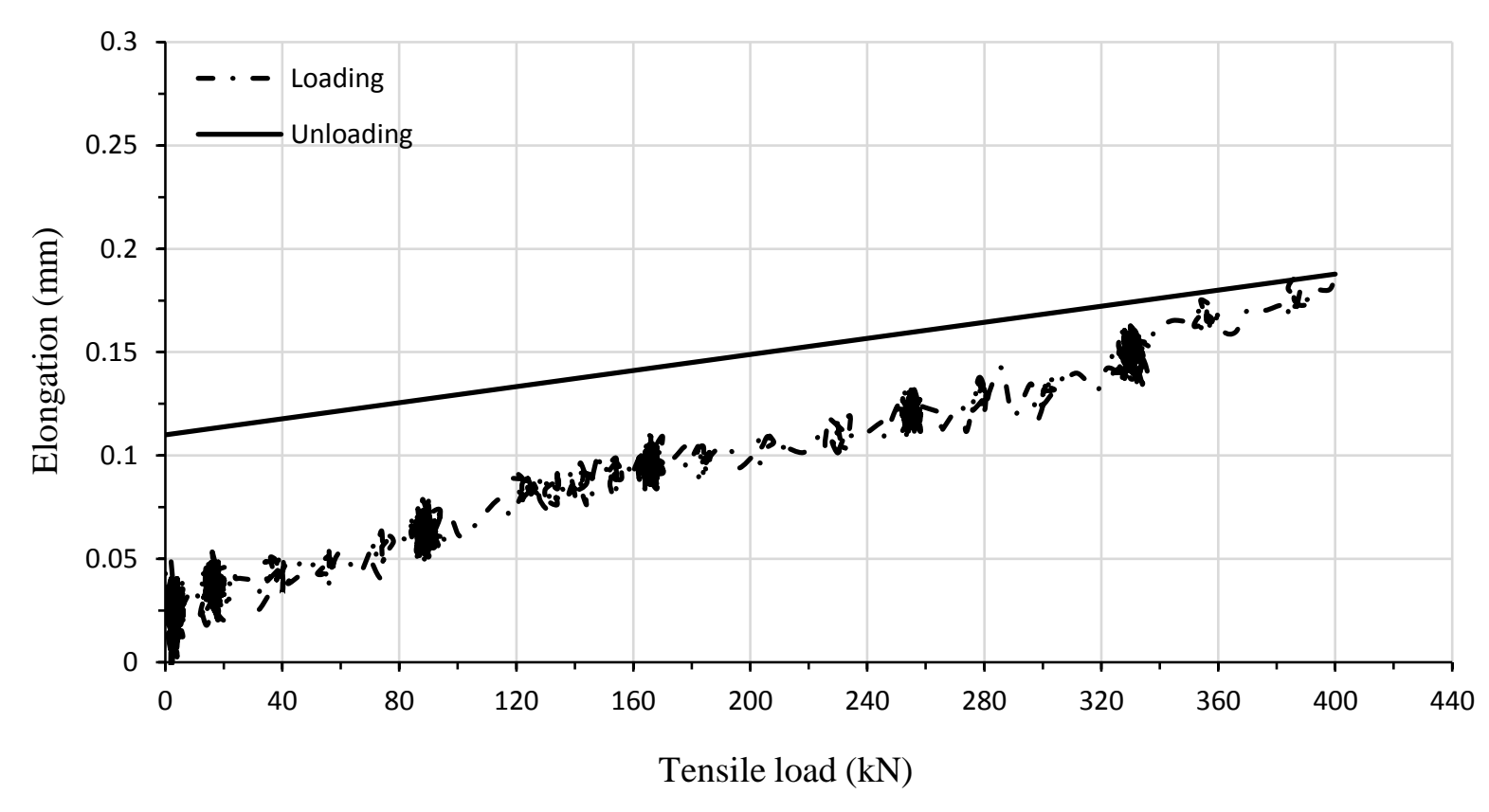

Figure 4-20: Tensile load versus horizontal displacement of the GFRP repaired NC2 specimen with respect to mid-span (crack location)

Table 4-7 summarizes the performance of the GFRP repaired NC2 specimen in terms of strength, crack width and leakage behavior and compares to the $\mathrm{NC} 2$ panel performance before the repair. By incrementally applying the tensile load on NC2 panel, a major crack formed at mid-span at load of $114 \mathrm{kN}$, along with crack width as wide as $0.3 \mathrm{~mm}$, which was followed by a high rate of leakage at mid-span crack. However, after repairing the NC2 specimen with 2 layers of GFRP sheets, the performance of NC panel in terms of strength, crack width, leakage was significantly improved. Even at high level of loading up to $400 \mathrm{kN}$, no failure was observed in GFRP laminate due to its high tensile strength. Moreover, in spite of existence and widening of the cracks at midspan, no leakage was observed in the GFRP repaired specimen which is an indication of the high effectiveness and efficiency of the GFRP laminates in waterproofing and recovering the structural strength of the cracked RC structures exposed to water pressure and monotonic direct tensile 
loading such as water tanks. It is worth-mentioning that GFRP laminates also had a significant restraining effect on crack width of NC panel especially at high levels loading.

Table 4-7: Comparison of the performance of NC2 panel before and after repair using GFRP

\begin{tabular}{|c|c|c|c|c|}
\hline & $\begin{array}{l}\text { Tensile load } \\
\qquad(\mathrm{kN})\end{array}$ & $\begin{array}{l}\text { Mid-span crack } \\
\text { width }(\mathrm{mm})\end{array}$ & $\begin{array}{l}\text { Water pressure } \\
\text { (psi) }\end{array}$ & $\begin{array}{l}\text { Leakage rate } \\
\qquad(\mathrm{ml} / \mathrm{hr})\end{array}$ \\
\hline \multirow{4}{*}{ Before repair } & \multirow{4}{*}{114} & \multirow{4}{*}{0.3} & 4 & 3996 \\
\hline & & & 8 & 8103 \\
\hline & & & 12 & 10389 \\
\hline & & & 16 & 12710 \\
\hline \multirow{4}{*}{ GFRP repair } & \multirow{4}{*}{$\mathrm{NA}^{*}$} & \multirow{4}{*}{0.78} & 4 & No leakage \\
\hline & & & 8 & No leakage \\
\hline & & & 12 & No leakage \\
\hline & & & 16 & No leakage \\
\hline
\end{tabular}

* Test stopped at $400 \mathrm{kN}$ due to rebar yielding, no failure in GFRP and no leakage was observed.

\section{4-2-3 Performance of ECC fly ash repaired RC panel}

Monotonic tensile loading was applied on the NC panel repaired with a $50 \mathrm{~mm}$ layer of ECC fly ash with the same procedure as outlined in Chapter 3. Direct tensile loading applied to the specimen through the hydraulic jacks with an increment of $10 \mathrm{kN}$. The load kept increasing until leakage initiation observed at mid-span crack. The first micro-cracks started to occur in ECC repair layer at midspan (where the crack existed in $\mathrm{NC}$ panel) at the load of $83 \mathrm{kN}$. However, no significant leakage was observed at this level of loading. Increasing the monotonic tensile loading resulted in a low leakage rate at $125 \mathrm{kN}$ at mid-span. Table 4-8 summarizes the tensile load and crack width in NC panel and ECC fly ash repair layer at each stage of loading. The residual crack width in NC3 panel before the repair was $0.09 \mathrm{~mm}$ and was increased by incrementally applying the tensile loading on the repaired sample. At loading level of $83 \mathrm{kN}$, at which ECC slag repair layer started to develop micro-cracks with average crack width of 50 Microns, crack width in the NC panel reached $0.29 \mathrm{~mm}$. The load at which leakage initiated, which is an important parameter 
in this study, was equal to $125 \mathrm{kN}$ along with crack width of 0.05 to $0.1 \mathrm{~mm}$ in the ECC fly ash repair layer and $0.37 \mathrm{~mm}$ in the $\mathrm{NC}$ panel.

Table 4-8: Tensile load and crack width in NC panel and ECC repair layer

\begin{tabular}{|c|c|c|}
\hline \multirow{2}{*}{ Tensile load $(\mathrm{kN})$} & \multicolumn{2}{|c|}{ Crack width $(\mathrm{mm})$} \\
\cline { 2 - 3 } & ECC fly ash repair layer & NC panel \\
\hline 0 & 0 & 0.09 \\
\hline 20 & 0 & 0.13 \\
\hline 40 & 0 & 0.18 \\
\hline 60 & 0 & 0.23 \\
\hline 83 & 0.05 & 0.29 \\
\hline 100 & 0.05 & 0.33 \\
\hline 125 & $0.05-0.10$ & 0.37 \\
\hline
\end{tabular}

At this stage of loading, the leakage rate was measured under different water pressures. Figure 421 presents the leakage rate in NC3 repaired with ECC fly ash at the loading stage of $125 \mathrm{kN}$ under various water pressures of $4,8,12$, and 16 psi and compares to the leakage rate under the same water pressures in NC3 before repair at the loading stage of $118 \mathrm{kN}$. A significant reduction in the leakage rate was observed after repairing the NC3 specimen with ECC fly ash even at a higher level of loading. To illustrate, leakage rate of the NC3 specimen recorded as 4589, 9262, 11556, and $15295 \mathrm{ml} / \mathrm{hr}$ before the repair under water pressures of 4, 8, 12, and $16 \mathrm{psi}$, respectively, while the leakage rate considerably decreased to the values of $67,145,179$, and $215 \mathrm{ml} / \mathrm{hr}$ under 4,8 , 12, and 16 psi water pressures in NC1 repaired with ECC fly ash. Considerably lower leakage rate in NC3 panel repaired with ECC fly ash compared to NC3 panel before repair can be attributed to the formation of micro-cracks in ECC layer. Therefore, water should penetrate through the full depth of micro-cracks in ECC layer, passing through the bond between the ECC layer and NC panel, and travel the full depth of the NC panel to leak from the bottom face of the NC panel. This 
mechanism potentially reduced the leakage rate of the NC3 repaired with ECC fly ash. Figure 422 illustrates low leakage rate of NC panel repaired using ECC fly ash.

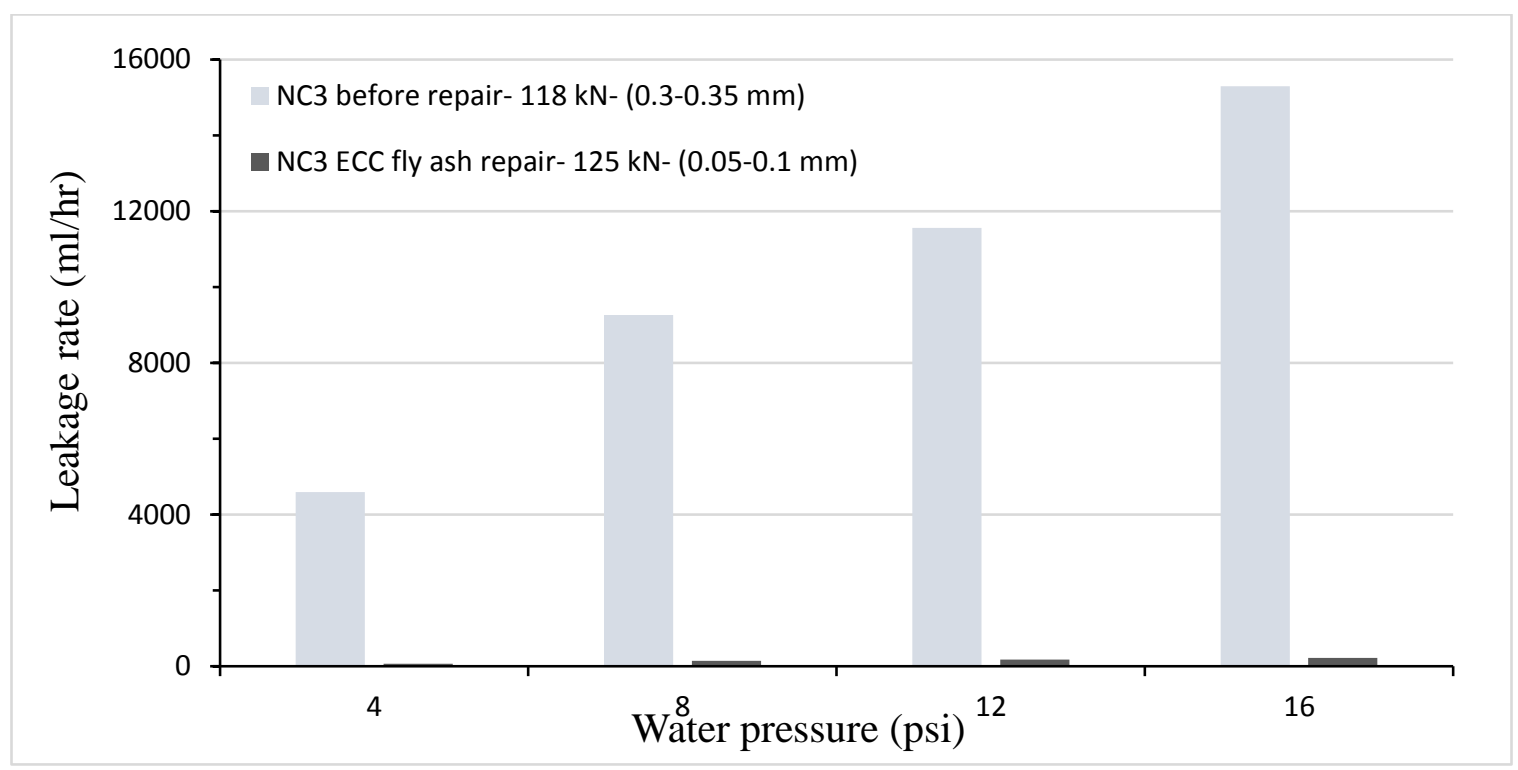

Figure 4-21: Comparison of the leakage behavior of NC3 before and after repair using ECC fly ash

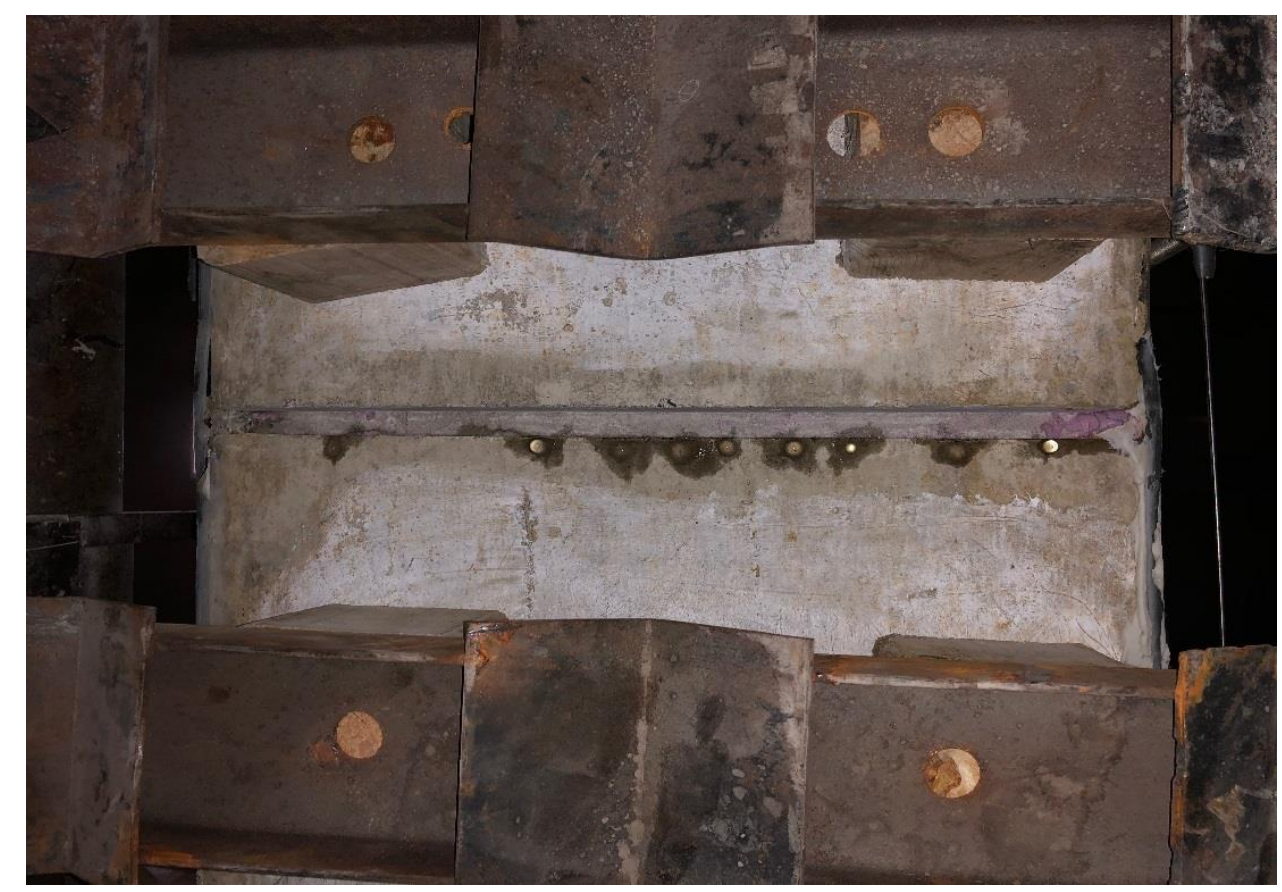

Figure 4-22: Leakage of the NC3 specimen repaired using ECC fly ash under 8 psi water pressure (bottom face) 
Tensile steel strain development in NC3 repaired with ECC fly ash is shown in Figure 4-23. Steel strain started from the residual strain in the rebar from previous stage of loading (before repair). Tensile strain gradually increased in the rebar by incrementally applying the direct tensile loading on the specimen. At this level of loading, NC panel is cracked at midspan and has no contribution in carrying the tensile load, while ECC repair layer is uncracked and cooperates in carrying the tension along with the rebar. Therefore, the tensile load is transferred from the rebar embedded in the NC panel to the ECC repair layer through the bond between the rebar and NC panel and the bonding between the NC panel and ECC repair layer. At 1/6-span, where two steel strain gauges are placed, both NC panel and ECC repair layer are uncracked and active in tension. Tensile strain in the rebar increased more rapidly by the formation of micro-cracks in ECC repair layer at midspan.

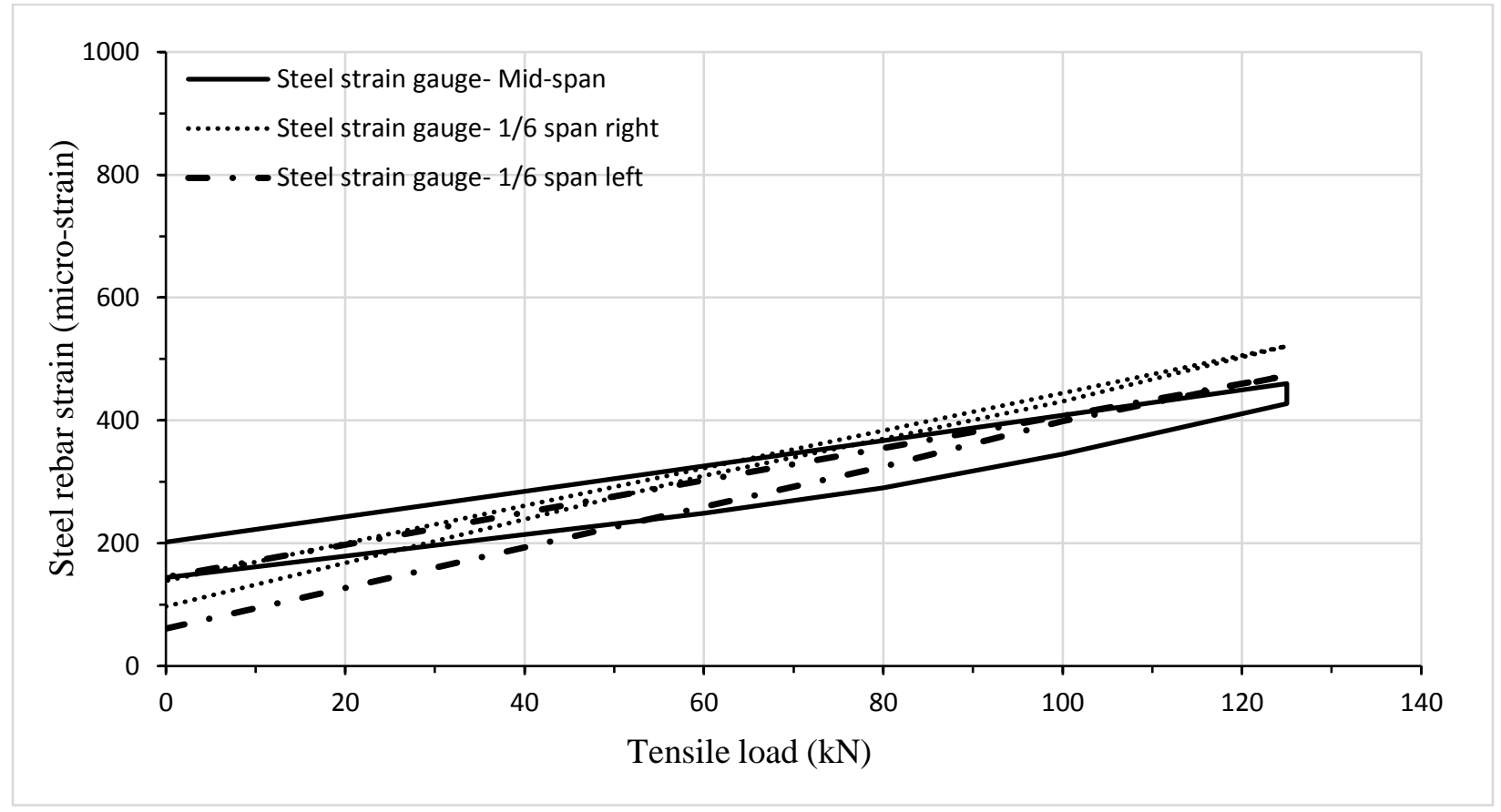

Figure 4-23: Tensile steel strain development in NC3 repaired using ECC fly ash

Figures 4-24 illustrates the load versus relative horizontal displacement of the repaired NC3 panel with respect to the mid-span (crack location). As the LVDTs were attached to the ECC repair layer, 
horizontal displacement started from zero, not including the residual crack width in NC3 panel from previous stage of loading. The horizontal displacement of the ECC layer increased by the formation of micro-cracks in ECC fly ash repair layer due to increasing the tensile loading. Gradual formation of micro-cracks in ECC layer with limited width of 0.05 to $0.1 \mathrm{~mm}$ resulted in horizontal displacement of $0.21 \mathrm{~mm}$ in ECC layer with respect to the mid-span.

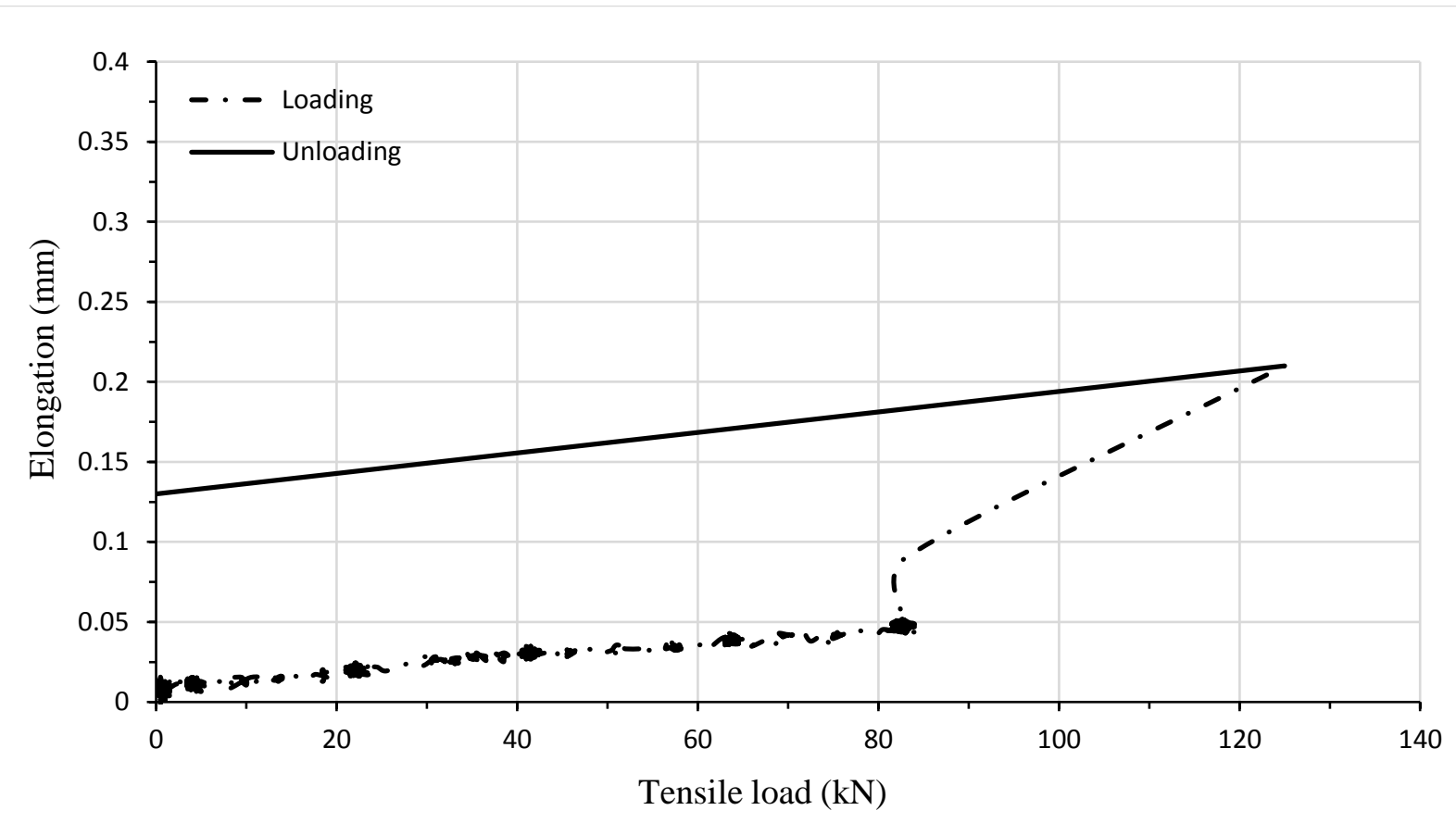

Figure 4-24: Tensile load versus relative horizontal displacement of the NC3 panel with respect to the mid-span (crack location)

After recoding the leakage rate of the NC3 panel repaired with ECC fly ash, water pressure in the chamber was set to 7 psi to investigate the self-healing behavior of the $50 \mathrm{~mm}$ ECC repair layer on top of the NC panel under a constant load of $125 \mathrm{kN}$ and fixed water pressure of 7 psi. For this purpose, leakage rate of the $\mathrm{NC1}$ repaired with ECC fly ash was recorded continuously to achieve a constant leakage rate or to attain $100 \%$ self-healing of the tension cracks. As the time prolonged, tension induced crack started to heal and resulted in reducing the leakage rate. In short-term investigation of the self-healing, the amount of water penetrated through the full depth of the 
tension cracks in ECC fly ash repair layer and NC panel and leaked from the bottom surface on the NC panel was measured over a one-hour time intervals. Short-term self-healing of the NC3 repaired with ECC fly ash during the first 35 hours is shown in Figure 4-25. The leakage rate reduced by $75 \%$ from $138 \mathrm{ml} / \mathrm{hr}$ to $35 \mathrm{ml} / \mathrm{hr}$ during the first 35 hours. A sharp drop was observed in leakage rate during the first 8 hours in which the leakage rate decreased by $50 \%$ from $138 \mathrm{ml} / \mathrm{hr}$ to $68 \mathrm{ml} / \mathrm{hr}$. The rate of leakage reduction subsided as the time prolonged which is due to reduction in self-healing capacity of ECC repair layer and NC panel over time. This can be explained by the fact that there are less available cementitious materials over time to participate in self-healing of the concrete panels.

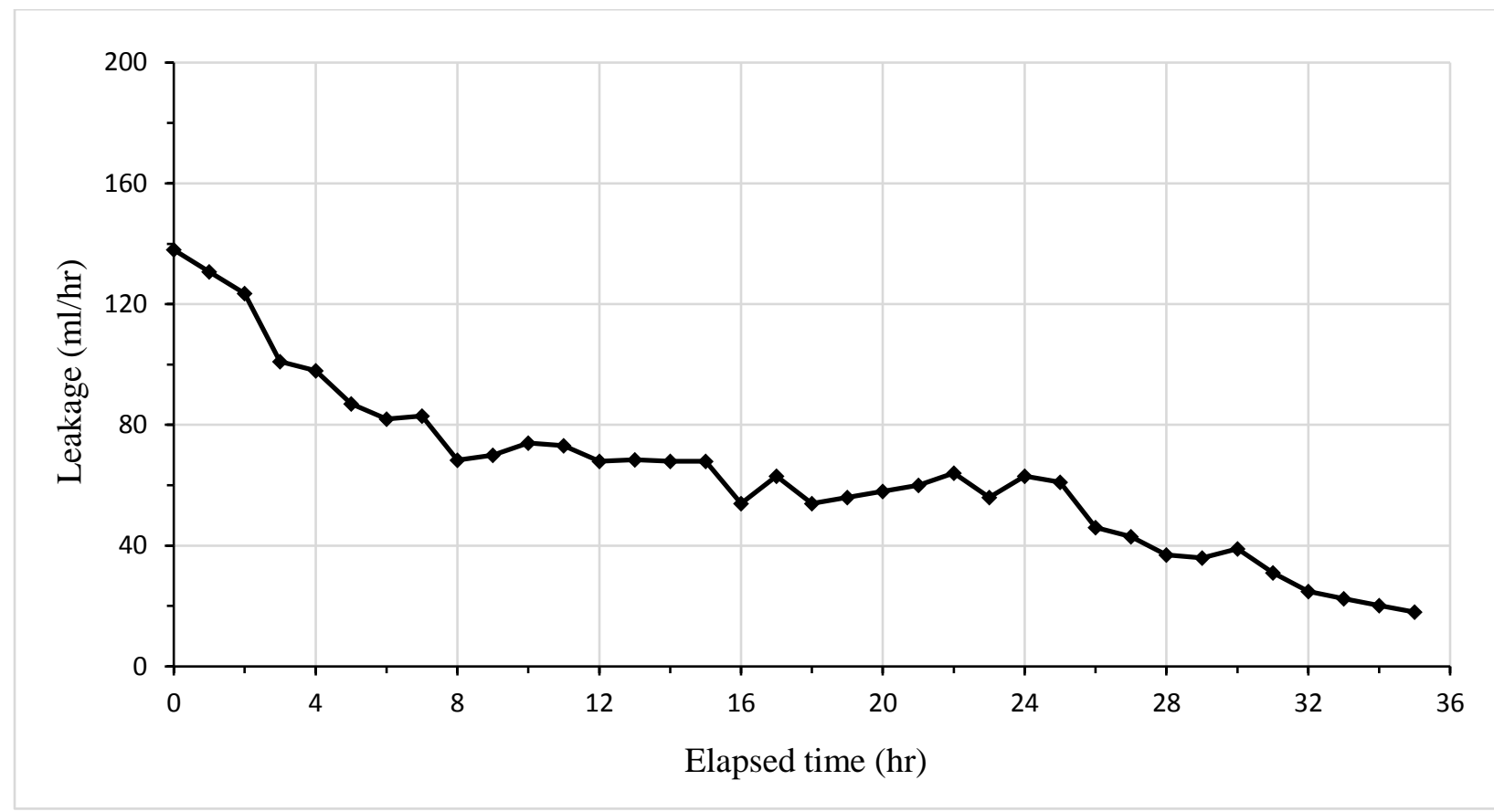

Figure 4-25: Short-term self-healing of NC3 panel repaired using ECC fly ash

Figure 4-26 illustrates the long-term self-healing of the NC3 repaired with ECC fly ash over the period of 7 days. During the first 35 hours, leakage was recorded continuously each hour, while during the period of time between 35 hours up to 7 days (168 hours), leakage rate was measured 
for one hour in each 6 hours. Leakage rate in the NC3 repaired with ECC fly ash reduced to zero after 7 days of monitoring the leakage rate which indicates the water flow with pressure of $7 \mathrm{psi}$ was completely blocked by the self-healing products. Blockage of the water flow could occur either in the ECC repair layer or in the interface between ECC repair layer and NC panel. Achieving the zero-leakage rate does not necessarily indicates $100 \%$ self-healing of the full depth crack in ECC repair layer and NC panel. It is worth-mentioning that obstruction created by selfhealing products in partial depth of the section or in the interface of ECC repair layer and NC panel can prevent the leakage and make flow of water through the depth of the section impossible. Moreover, use of fly ash in ECC mix design as supplementary cementitious material resulted in smaller crack width along with higher self-healing capacity of the ECC fly ash repair layer compared to the ECC slag repair layer.

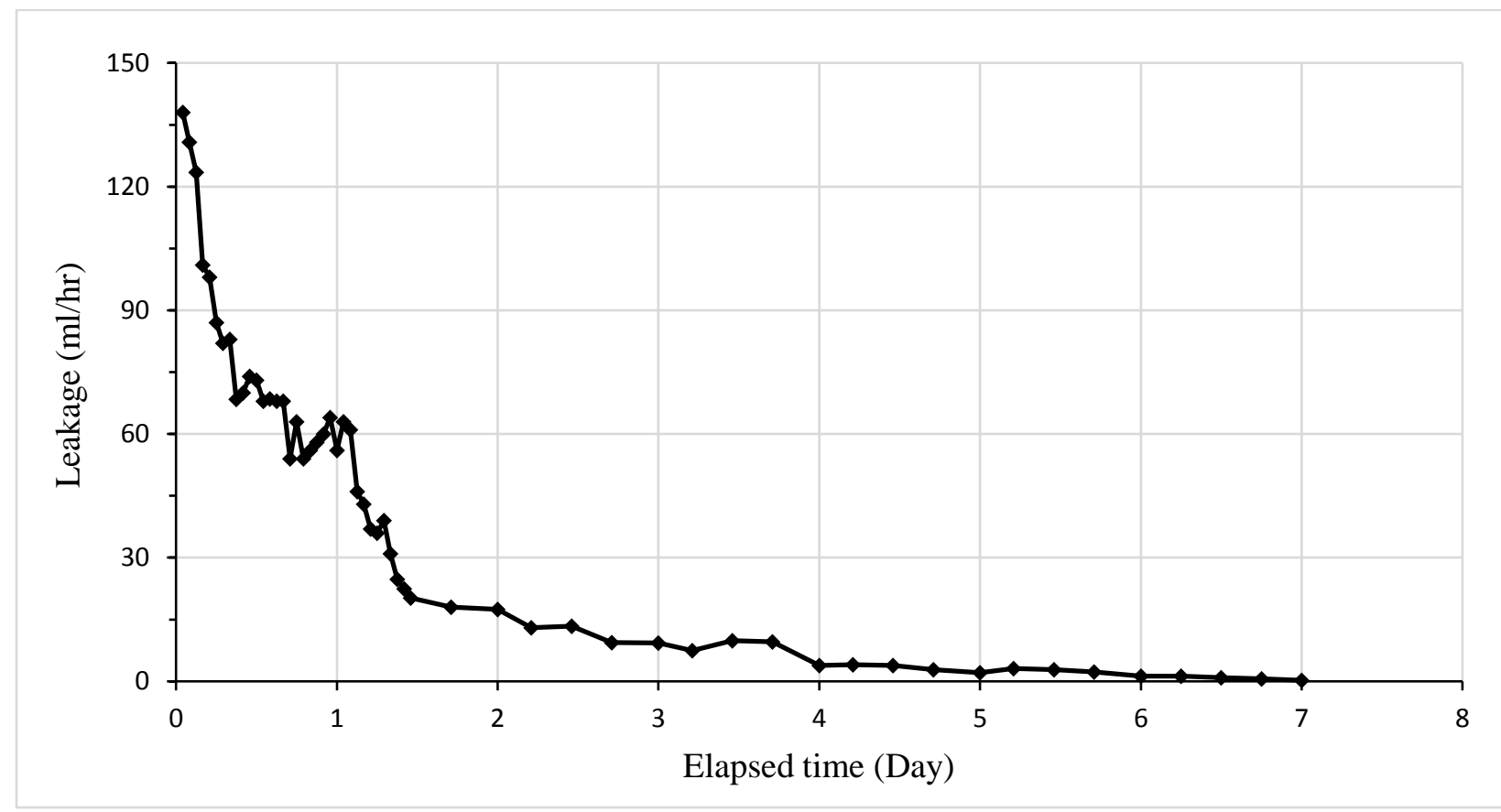

Figure 4-26: Long-term self-healing of NC3 panel repaired using ECC fly ash 
Table 4-9 summarizes the performance of the NC3 repaired with ECC fly ash in terms of strength, crack width and leakage behavior and compares to the NC3 panel performance before the repair. Applying the direct tensile loading on the NC3 panel before repair resulted in formation a major crack at mid-span of the specimen at load of $118 \mathrm{kN}$, along with crack width of $0.3-0.35 \mathrm{~mm}$, which was followed by a high rate of leakage. However, applying a $50 \mathrm{~mm}$ ECC fly ash repair layer on top of the NC panel resulted in a significant lower leakage rate at the tensile load of 125 $\mathrm{kN}$, by formation of micro-cracks with maximum crack width of 50 to 100 Microns. The results show that the $50 \mathrm{~mm}$ ECC fly ash repair layer significantly improved the cracking and leakage behavior of the NC panel. Moreover, ECC fly ash repair layer successfully recovered the structural strength of the cracked NC panel. This can be explained by multiple micro-cracking behavior of ECC along with strain hardening and high tensile strain capacity, which makes ECC it an ideal choice for repairing RC structures including water tanks in which superior cracking and leakage behavior is of prime importance. It is worth-mentioning that use of fly ash F as supplementary cementitious material contributes to the multiple micro-cracking behavior of ECC and its high self-healing ability. Figure 4-27 illustrates the difference between the leakage rate of the NC panel before repair and after repair with ECC fly ash.

Table 4-9 Comparison of the performance of NC3 before and after repair using ECC fly ash

\begin{tabular}{|c|c|c|c|c|}
\hline & $\begin{array}{c}\text { Tensile load } \\
(\mathrm{kN})\end{array}$ & $\begin{array}{c}\text { Crack width } \\
(\mathrm{mm})\end{array}$ & $\begin{array}{c}\text { Water pressure } \\
\text { (psi) }\end{array}$ & $\begin{array}{c}\text { Leakage rate } \\
(\mathrm{ml} / \mathrm{hr})\end{array}$ \\
\hline \multirow{4}{*}{ Before repair } & \multirow{4}{*}{118} & \multirow{4}{*}{$0.3-0.35$} & 4 & 4589 \\
\hline & & & 8 & 9262 \\
\hline & & & 12 & 11556 \\
\hline & & & 16 & 15295 \\
\hline \multirow{4}{*}{ ECC fly ash repair } & \multirow{4}{*}{125} & \multirow{4}{*}{$0.05-0.1$} & 4 & 67 \\
\hline & & & 8 & 145 \\
\hline & & & 12 & 179 \\
\hline & & & 16 & 215 \\
\hline
\end{tabular}




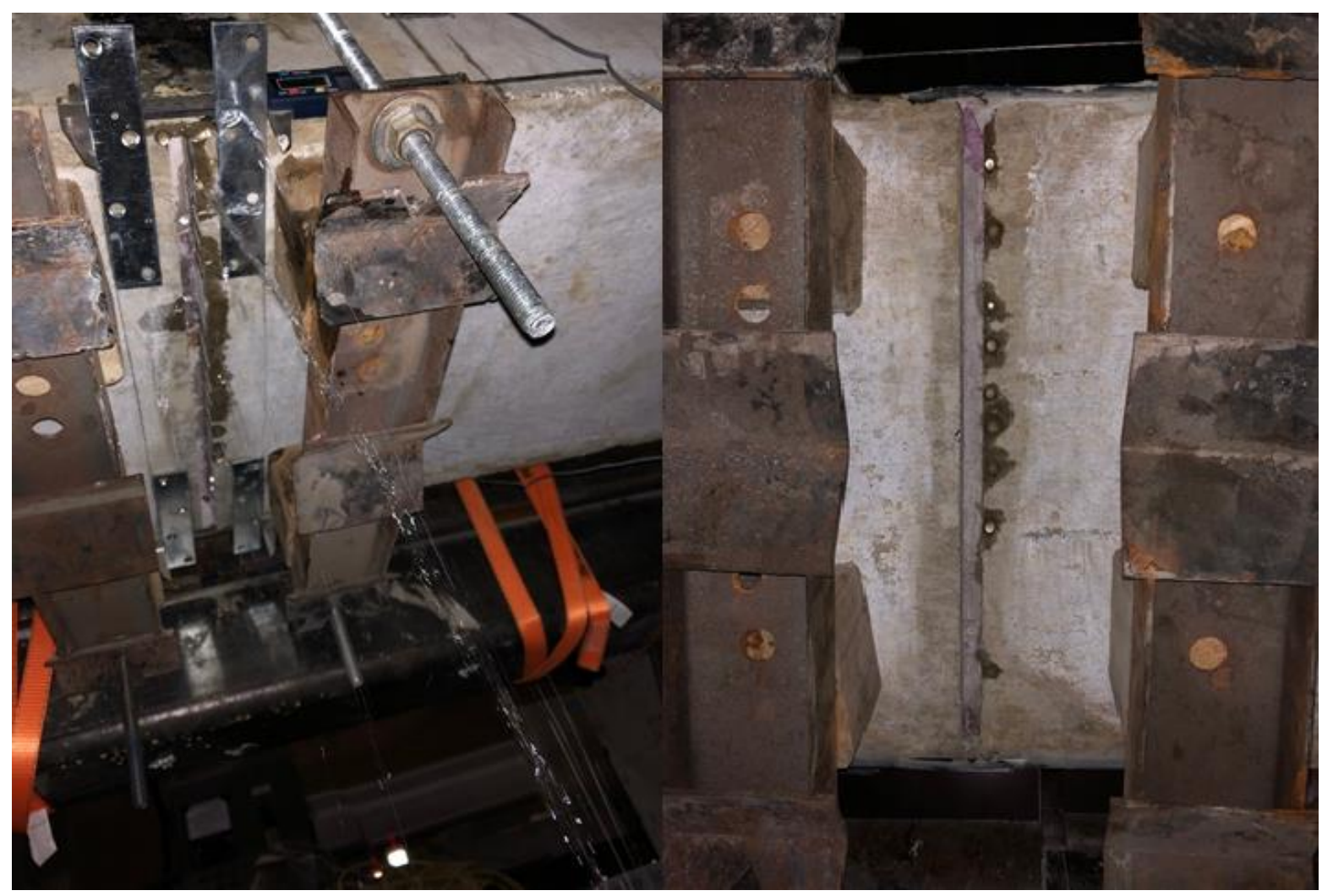

(a)

(b)

Figure 4-27: Leakage of the NC panel before and after repair using ECC fly ash a) Before repair b) After repair

\section{4-2-4 Performance of epoxy injection repaired $\mathrm{RC}$ panel}

Monotonic tensile loading was applied on the NC4 panel repaired with high-strength epoxy injection according to the procedure as outlined in Chapter 3. Direct tensile loading applied to the specimen through the hydraulic jacks with increments of $10 \mathrm{kN}$. The load kept increasing until failure of the epoxy and leakage initiation at crack location (mid-span). Failure of epoxy at crack location occurred at tensile load level of $256 \mathrm{kN}$, along with a large crack as wide as 0.65-0.7 mm, which was immediately followed by high rate leakage. Formation of a large crack as well as high leakage can be attributed to the sudden brittle failure of the epoxy at mid-span (crack location). Table 4-10 and Figure 4-28 illustrate the crack widths at mid-span, 1/6-span from both ends, and end-spans of the NC4 panel repaired with epoxy at different stages of loading. 
Table 4-10 Summary of the load and crack width of NC4 specimen after repair with epoxy

\begin{tabular}{|c|c|c|c|}
\hline \multirow{2}{*}{ Tensile load $(\mathrm{kN})$} & \multicolumn{3}{|c|}{ Crack width (mm) } \\
\cline { 2 - 4 } & Mid-span & $1 / 6$-spans & End-spans \\
\hline 0 & 0.22 & 0 & 0 \\
\hline 50 & 0.22 & 0 & 0 \\
\hline 100 & 0.22 & 0 & 0 \\
\hline 150 & $0.22-0.23$ & 0 & 0 \\
\hline 178 & $0.22-0.23$ & 0 & $0.2-0.25$ \\
\hline 200 & $0.22-0.23$ & 0 & 0.3 \\
\hline 220 & $0.22-0.23$ & 0 & 0.35 \\
\hline 240 & 0.23 & $0.1-.15$ & $0.4-0.45$ \\
\hline 256 & $0.65-0.7$ & 0.2 & 0.45 \\
\hline 280 & 0.75 & $0.2-0.25$ & $0.5-0.55$ \\
\hline 300 & $0.8-0.85$ & 0.25 & 0.55 \\
\hline 320 & 0.9 & 0.3 & 0.6 \\
\hline 340 & 1.0 & $0.35-0.4$ & $0.6-0.65$ \\
\hline 360 & $1.05-1.1$ & 0.4 & 0.65 \\
\hline 380 & $1.1-1.15$ & 0.45 & \\
\hline & & & \multicolumn{3}{|c|}{} \\
\hline & & 0.45 & 0.45 \\
\hline
\end{tabular}

It can be observed that the mid-span crack width increased from the residual crack width of 0.22 $\mathrm{mm}$, from previous loading, to $1.1-1.15 \mathrm{~mm}$ at $380 \mathrm{kN}$. By incrementally increasing the tensile loading, at the loading stage of $178 \mathrm{kN}$, bond slip occurred between the rebar and concrete at both ends of the specimen simultaneously resulted in a crack as wide as $0.2 \mathrm{~mm}$ propagating toward top and bottom surfaces of the NC panel. At this stage of loading, no cracking occurred at 1/6-span and no significant change was observed at mid-span maintaining the same residual crack width of $0.22 \mathrm{~mm}$. By further increasing the tensile load, developed cracks at both ends propagated along the longitudinal direction of the specimen toward mid-spam. At loading stage of $240 \mathrm{kN}$, cracks propagated in transverse direction (extended to the entire width) causing cracks as wide as 0.1$0.15 \mathrm{~mm}$ at distance of $240 \mathrm{~mm}$ from the north end and $265 \mathrm{~mm}$ from the south end of the specimen (almost at 1/6 of the span). At this stage of loading, epoxy was still capable of carrying the tensile 
load resulted in keeping the mid-span crack at almost residual value of 0.22 to $0.23 \mathrm{~mm}$, while crack widths at end-span reached $0.35 \mathrm{~mm}$. It is interesting to note that up to this stage of loading, no failure was observed in epoxy at crack location and epoxy was still active in tension, carrying the tensile load, and preventing crack width growth and leakage initiation. Finally, by enhancing the tensile load to $256 \mathrm{kN}$, epoxy failure occurred. Due to brittle nature of the epoxy, crack width substantially increased to the value of $0.65-0.7 \mathrm{~mm}$ followed by a high rate of leakage at mid-span. At this stage of loading, crack widths at 1/6-span and end-span were $0.2 \mathrm{~mm}$ and 0.4-0.45 mm, respectively. Further increase in tensile load resulted in widening the crack at mid-span, 1/6-span, and end-span. Loading of the specimen stopped at loading stage of $380 \mathrm{kN}$ due to safety reasons, as the tensile strain in the rebar exceeded the yielding strain. Crack width at mid-span, 1/6-span, and end-span reached $1.1-1.15 \mathrm{~mm}, 0.45 \mathrm{~mm}$, and $0.65 \mathrm{~mm}$, respectively, at tensile load of 380 $\mathrm{kN}$.

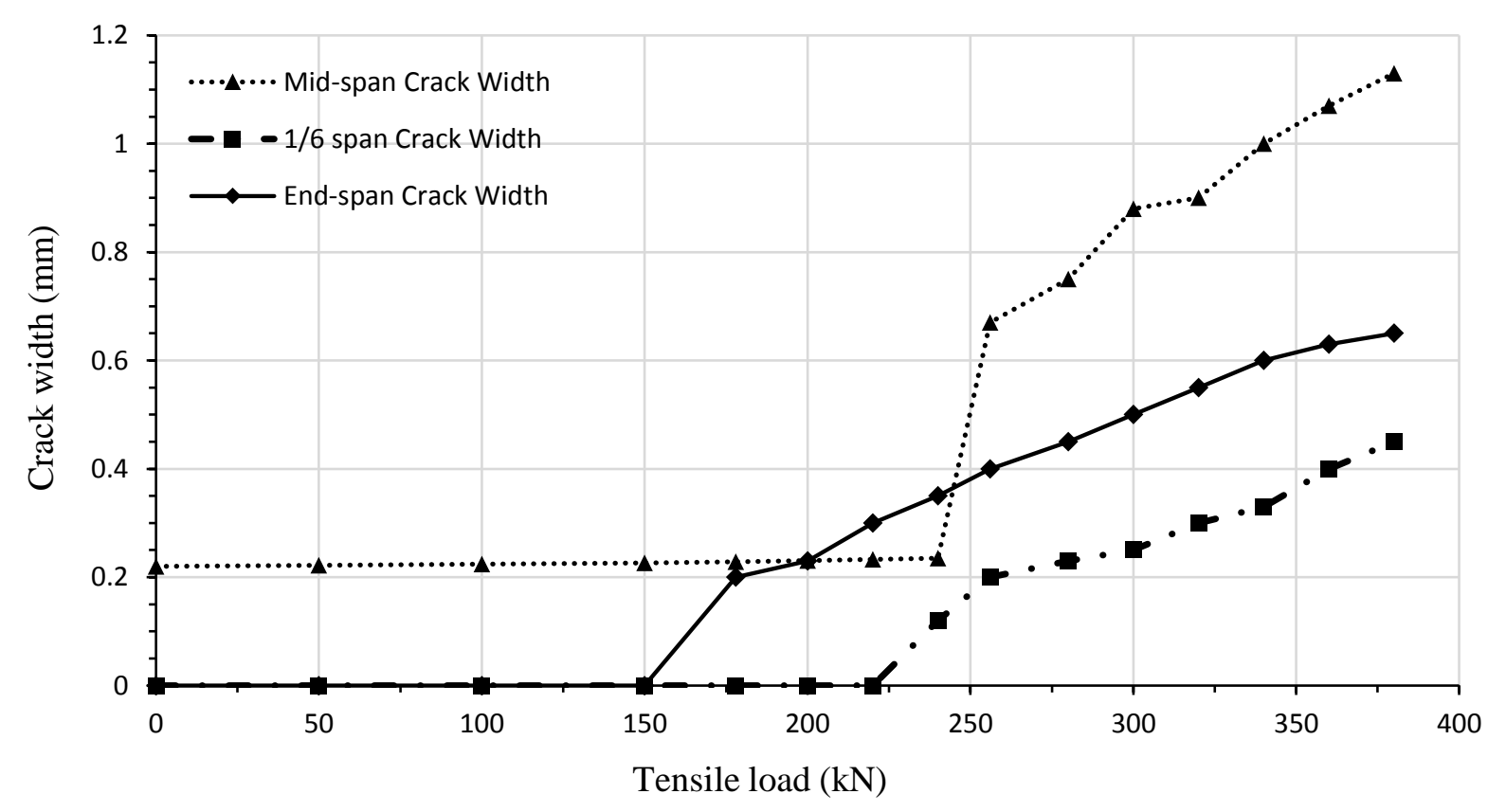

Figure 4-28: Tensile load and crack width of the NC4 specimen repaired using epoxy 
At this stage of loading, the leakage rate was measured under different water pressures. Figure 429 shows the leakage rate in NC4 panel repaired using epoxy at the loading stage of $256 \mathrm{kN}$ under various water pressures of $4,8,12$, and 16 psi and compares to the leakage rate under the same water pressures in NC4 Panel before repair at the loading stage of $127 \mathrm{kN}$. A higher leakage rate was observed in NC4 panel repaired with epoxy compared to NC4 panel before repair. This can be explained by the higher tensile load and crack width at which leakage rate was measured in epoxy repaired NC panel in comparison to NC panel before. It is evident that epoxy exhibited considerable bond strength and tensile strength and successfully increased the load bearing capacity of the NC panel under direct tensile loading. However, due to brittle nature of the epoxy, failure of the epoxy, which occurred at high levels of tensile load, resulted in a large crack width followed by a high leakage rate. To illustrate, the leakage of the NC4 panel before repair was measured at 5404, 10614,13872, and $17038 \mathrm{ml} / \mathrm{hr}$ under water pressures of 4, 8, 12, and 16 Psi at tensile loading of $127 \mathrm{kN}$ along with mid-span crack width of $0.35-0.4 \mathrm{~mm}$. However, higher leakage rate of $7864,16354,23155$, and $31357 \mathrm{ml} / \mathrm{hr}$ was recorded under the same water pressures at loading stage of $256 \mathrm{kN}$ along with crack width of $0.65-0.7 \mathrm{~mm}$ at mid-span crack. It is worthmentioning that higher leakage rate recorded after failure NC panel repaired with epoxy at $256 \mathrm{kN}$ compared to $\mathrm{NC}$ panel before repair leakage rate at $127 \mathrm{kN}$ does not necessarily mean that epoxy was unable to improve the leakage behavior of the NC panel, as the leakage rate of the epoxy repaired NC panel was recorded at considerably higher level of loading and crack width. Figure 430 shows the failure of the epoxy at mid-span crack followed by a high rate of leakage. 


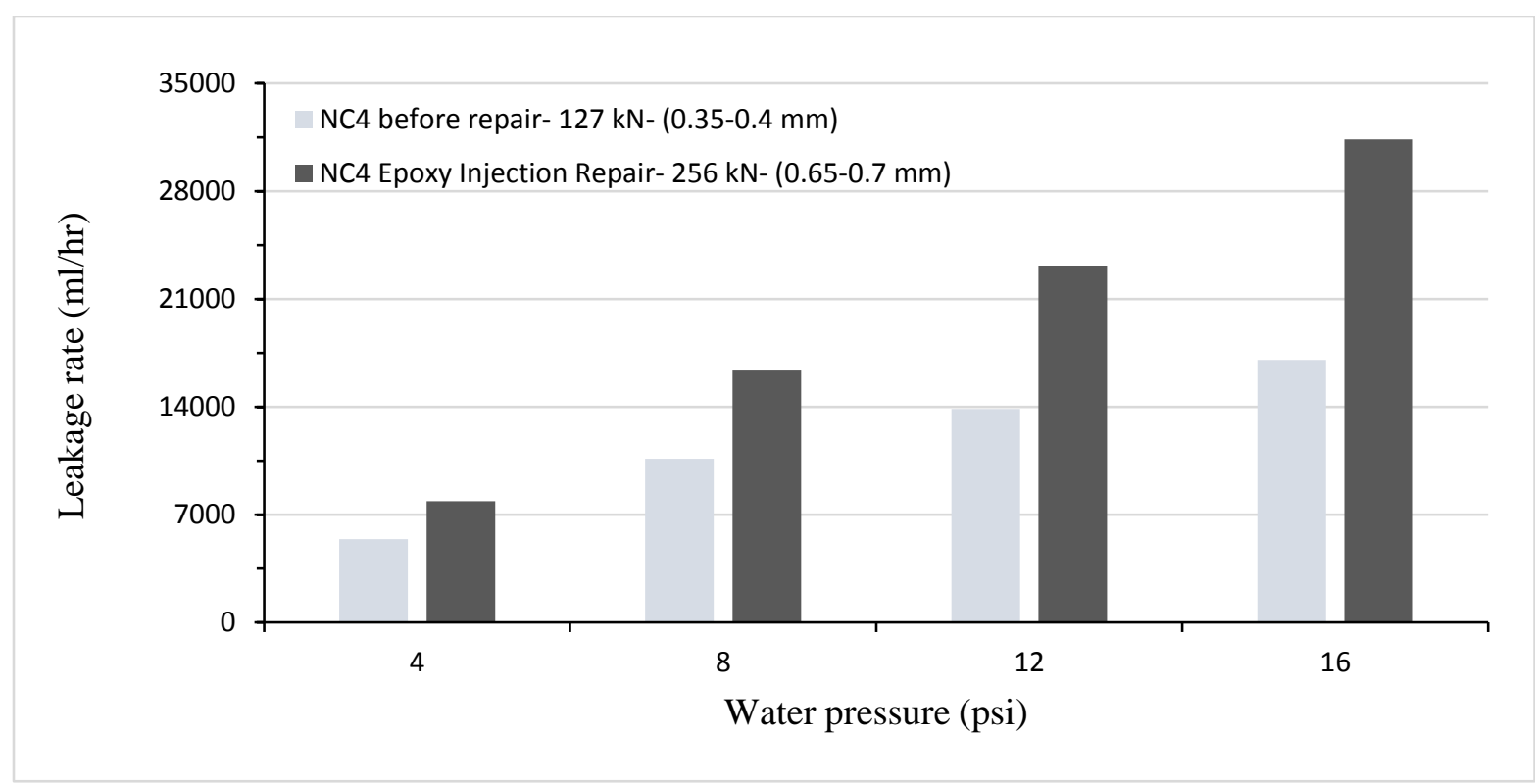

Figure 4-29: Comparison of the leakage behavior of NC4 before and after repair using epoxy

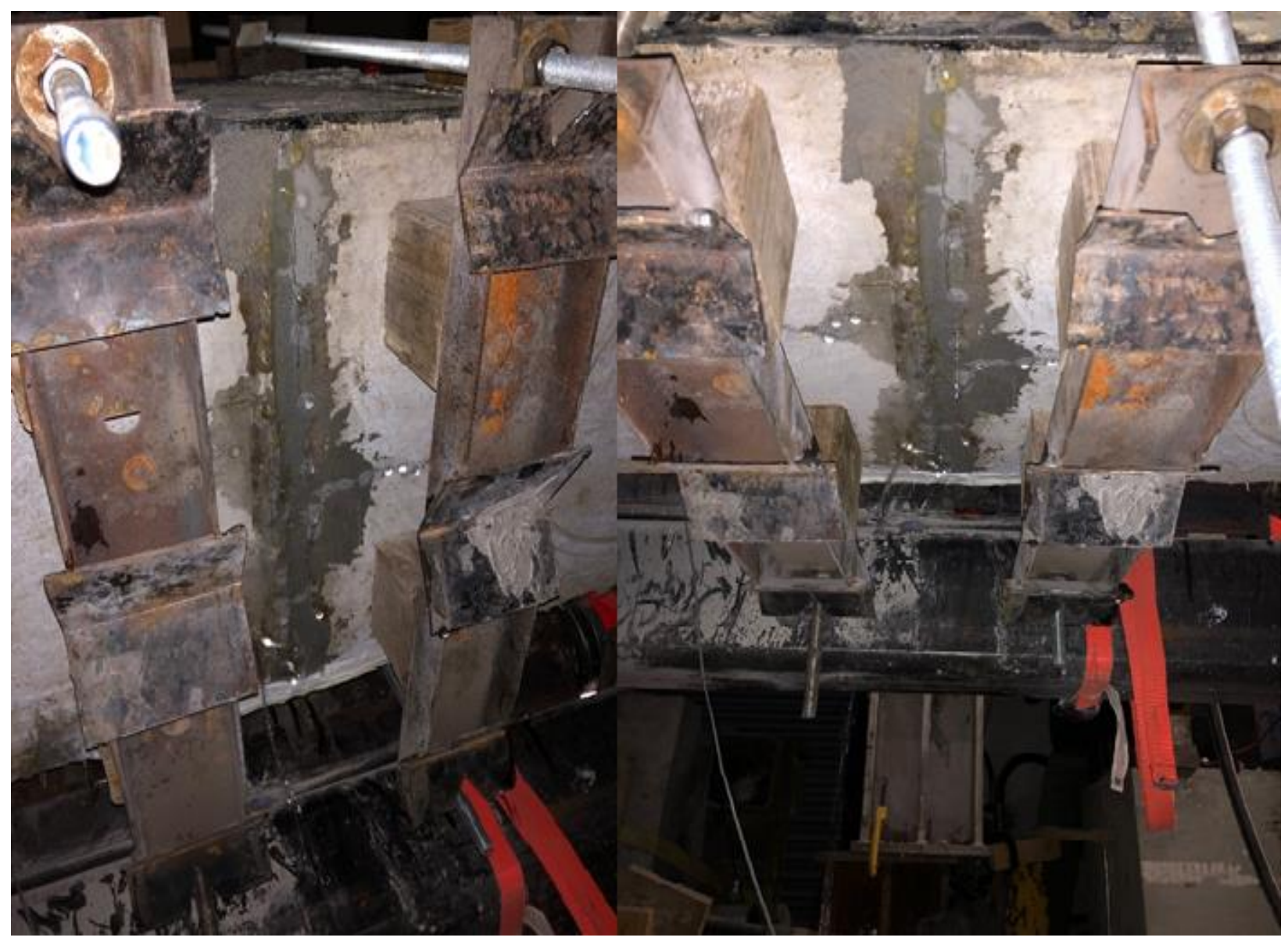

Figure 4-30: Brittle failure of the epoxy at crack location (mid-span) and high leakage rate 
In order to further investigate the cracking and leakage behavior of the epoxy repaired NC4 panel after failure of the epoxy at mid-span crack, the tensile loading was continued up to $380 \mathrm{kN}$. Table 4-11 summarizes the mid-span crack width and leakage rate of the NC panel under various water pressures of $4,8,12$, and $16 \mathrm{psi}$ in the range of tensile loading between $256 \mathrm{kN}$, at which failure of the epoxy occurred, and $380 \mathrm{kN}$, at which the test stopped due to safety reasons, as the tensile strain in the rebar exceeded the yielding strain. It can be observed that further increasing the tensile load resulted in increasing the mid-span, 1/3-span, and end-span crack width of the panel. As the leakage rate has an intimate relationship with leakage rate, at higher levels of tensile loading, which is accompanied with larger crack widths, a considerable increase in the leakage rate was observed. To illustrate, at loading level of $256 \mathrm{kN}$, mid-span crack was measured as $0.65-0.7 \mathrm{~mm}$ along with leakage rate of 7864, 16354, 23155, and $31357 \mathrm{ml} / \mathrm{hr}$ under water pressures of 4, 8, 12, and $16 \mathrm{psi}$, respectively, while substantially larger crack width of 1.1-1.15 mm along with considerably higher leakage rate of $12810,27625,38106$, and $50124 \mathrm{ml} / \mathrm{hr}$ under the same water pressures was recorded at tensile loading stage of $380 \mathrm{kN}$.

Figure 4-31 illustrates the tensile steel strain development in NC3 repaired with high-strength epoxy. Steel strain started from the residual strain in the rebar from previous stage of loading (before repair). Gradual increasing of the tensile strain in the rebar was observed by incrementally applying the direct tensile loading on the specimen. At initial levels of loading, epoxy is active in tension and contributes in carrying the tensile load. Therefore, tensile loading is transferred to the epoxy through the bond between the rebar and NC panel. Tensile strain in the rebar at mid-span experienced a sharp increase at loading stage of $256 \mathrm{kN}$, at which failure of the high-strength epoxy occurred. At this level of loading, epoxy is no longer capable of carrying the tensile load and the 
whole tensile is tolerated by the rebar. Sudden increase in the tensile strain of the rebar at load of $256 \mathrm{kN}$ can be attributed to the brittle failure of the epoxy at mid-span crack.

Table 4-11: Summary of the tensile load, crack width, and leakage rate of the high-strength epoxy repaired NC4 panel under various water pressures

\begin{tabular}{|c|c|c|c|}
\hline Tensile load $(\mathrm{kN})$ & $\begin{array}{l}\text { Mid-span crack } \\
\text { width (mm) }\end{array}$ & $\begin{array}{l}\text { Water pressure } \\
(\mathrm{psi})\end{array}$ & $\begin{array}{c}\text { Leakage rate } \\
(\mathrm{ml} / \mathrm{hr})\end{array}$ \\
\hline \multirow{4}{*}{256} & \multirow{4}{*}{$0.65-0.7$} & 4 & 7864 \\
\hline & & 8 & 16354 \\
\hline & & 12 & 23155 \\
\hline & & 16 & 31357 \\
\hline \multirow{4}{*}{280} & \multirow{4}{*}{0.75} & 4 & 8447 \\
\hline & & 8 & 17955 \\
\hline & & 12 & 25052 \\
\hline & & 16 & 33496 \\
\hline \multirow{4}{*}{300} & \multirow{4}{*}{$0.8-0.85$} & 4 & 9123 \\
\hline & & 8 & 20504 \\
\hline & & 12 & 28272 \\
\hline & & 16 & 38470 \\
\hline \multirow{4}{*}{320} & \multirow{4}{*}{0.9} & 4 & 10245 \\
\hline & & 8 & 22510 \\
\hline & & 12 & 30712 \\
\hline & & 16 & 42213 \\
\hline \multirow{4}{*}{340} & \multirow{4}{*}{1.0} & 4 & 11054 \\
\hline & & 8 & 24923 \\
\hline & & 12 & 34657 \\
\hline & & 16 & 45234 \\
\hline \multirow{4}{*}{360} & \multirow{4}{*}{$1.05-1.1$} & 4 & 12130 \\
\hline & & 8 & 26123 \\
\hline & & 12 & 36289 \\
\hline & & 16 & 47741 \\
\hline \multirow{4}{*}{380} & \multirow{4}{*}{$1.1-1.15$} & 4 & 12810 \\
\hline & & 8 & 27625 \\
\hline & & 12 & 38106 \\
\hline & & 16 & 50124 \\
\hline
\end{tabular}

Tensile load versus relative horizontal displacement of the NC4 panel with respect to the mid-span (crack location) is shown in Figure 4-32. The horizontal displacement of the specimen starts from 
zero and does not include the residual crack from previous steps of loadings (before repair). The horizontal displacement of the NC4 panel increased slowly by increasing the tensile loading applied on the specimen, followed by sudden sharp increase at tensile loading level of $256 \mathrm{kN}$ due to brittle failure of the epoxy and substantial widening of the mid-span crack.

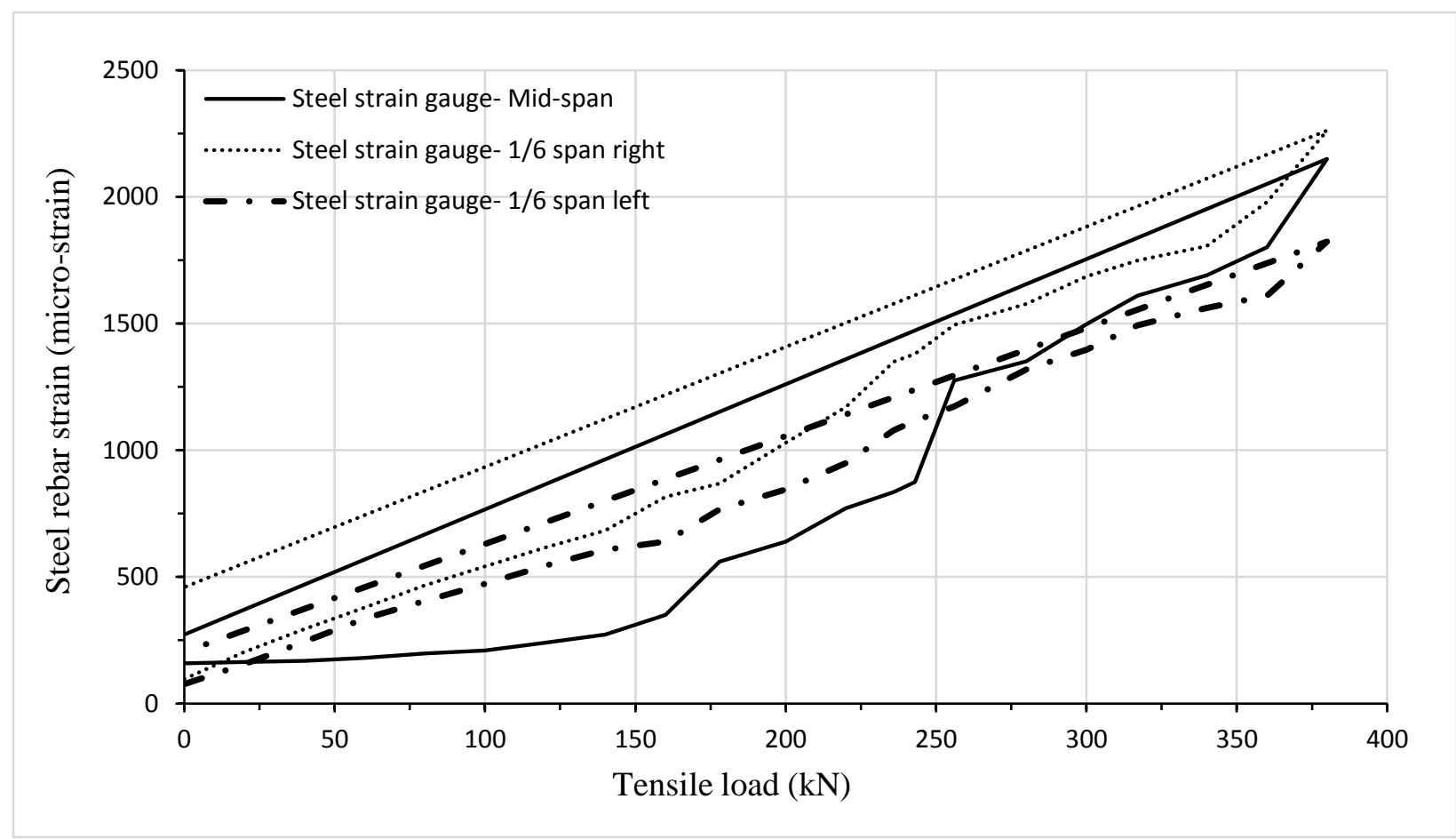

Figure 4-31- Tensile steel strain development in NC4 panel repaired using high-strength epoxy 


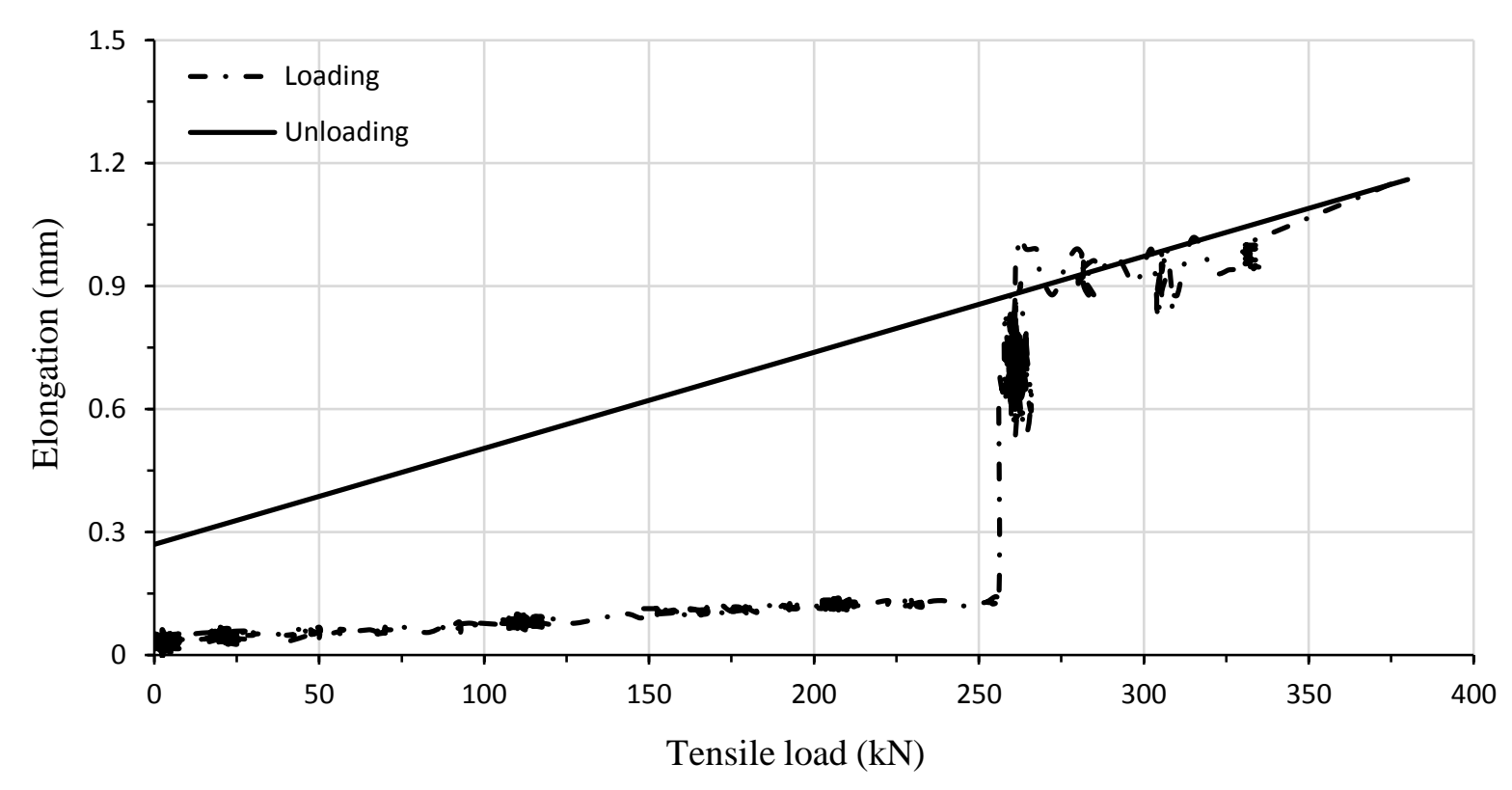

Figure 4-32: Tensile load versus relative horizontal displacement of the NC4 panel with respect to the mid-span (crack location)

Table 4-12 summarizes the performance of the high-strength repaired NC4 specimen in terms of strength, crack width and leakage behavior and compares to the NC4 panel performance before the repair. By incrementally applying the tensile load on NC4 panel before repair, a major crack formed at mid-span at load of $127 \mathrm{kN}$, along with crack width as wide as 0.35-0.4 mm, which was followed by a high rate of leakage at mid-span crack. However, repairing the mid-span crack of the NC4 panel with high-pressure injection of the high-strength epoxy significantly improved the tensile load bearing capacity of the panel. The tensile load at which leakage initiated increased considerably from $127 \mathrm{kN}$ before repair to $256 \mathrm{kN}$ after the repair. It is interesting to note that up to the loading stage of $256 \mathrm{kN}$, no failure was observed in epoxy at crack location and epoxy was still capable of carrying the tensile load and preventing crack width growth and leakage initiation. This can be attributed to the material properties of the epoxy including high tensile strength and bond strength several times stronger than concrete. Due to brittle nature of the epoxy, failure of the high-strength epoxy at high levels of loading resulted in a substantial increase of the crack 
width (up to $0.65-0.7 \mathrm{~mm}$ ), followed by a high rate of leakage at mid-span crack. High rate of leakage after failure of the epoxy can be explained by the intimate relationship between the crack width and leakage rate.

Epoxy injection is known as a local repair solution for cracked RC structures. High tensile and bond strength of the epoxy resulted in formation of cracks at other locations (1/6-span at $240 \mathrm{kN})$ before failure of the epoxy at mid-span crack (at $256 \mathrm{kN}$ ), which shows the effectiveness of the high-pressure injection of the epoxy in recovering the structural strength at the cracked section of the damaged RC member. However, cracks may occur at other locations under the same level of tensile loading, which requires repeating the repair program with epoxy injection. This may affect the efficiency of the epoxy injection solution for repair of RC structures especially in case of liquid containing structures in which leakage prevention is of prime importance.

Table 4-12 Comparison of the performance of NC4 panel before and after repair using highstrength epoxy

\begin{tabular}{|c|c|c|c|c|}
\hline & $\begin{array}{c}\text { Tensile load } \\
(\mathrm{kN})\end{array}$ & $\begin{array}{l}\text { Crack width } \\
(\mathrm{mm})\end{array}$ & $\begin{array}{l}\text { Water pressure } \\
(\mathrm{psi})\end{array}$ & $\begin{array}{c}\text { Leakage rate } \\
(\mathrm{ml} / \mathrm{hr})\end{array}$ \\
\hline \multirow{4}{*}{ Before repair } & \multirow{4}{*}{127} & \multirow{4}{*}{$0.35-0.4$} & 4 & 5404 \\
\hline & & & 8 & 10614 \\
\hline & & & 12 & 13872 \\
\hline & & & 16 & 17038 \\
\hline \multirow{4}{*}{ Epoxy injection repair } & \multirow{4}{*}{256} & \multirow{4}{*}{$0.65-0.7$} & 4 & 7864 \\
\hline & & & 8 & 16354 \\
\hline & & & 12 & 23155 \\
\hline & & & 16 & 31357 \\
\hline
\end{tabular}

\section{4-3 Comparison of the efficiency of different repair materials}

Table 4-13 summarizes the performance of the NC panel before repair and after repair with different repair materials in terms of strength, crack width and leakage behavior. As the tensile 
loading was incrementally applied on the NC panel before the repair, a major crack was formed at mid-span (notch location) at loading level of 114-127 kN along with a crack width of 0.3-0.4 mm, which was immediately followed by a high rate leakage. Similar behavior was observed for all the NC panels before repair which can be explained by brittle nature of NC.

By applying the monotonic tensile loading to the NC panel repaired with $50 \mathrm{~mm}$ of ECC slag, a low rate of leakage initiated at the load of $139 \mathrm{kN}$ by developing micro-cracks in ECC slag repair layer with maximum crack width of $0.15 \mathrm{~mm}$. ECC slag repair layer retained the structural strength and improved cracking and leakage behavior of the NC panel. This can be attributed to the multiple micro-cracking behavior of ECC along with its strain hardening and high tensile strain capacity. Use of slag as the supplementary cementitious material (SCM) resulted in higher strength as well as developing larger crack width in ECC repair layer in comparison with ECC fly ash.

$\mathrm{NC}$ panel repaired with $50 \mathrm{~mm}$ ECC fly ash repair layer experienced a very low rate of leakage at tensile load of $125 \mathrm{kN}$, by formation of micro-cracks with maximum crack width of 50 to 100 Microns. ECC fly ash repair layer significantly improved the cracking and leakage behavior of the NC panel. Moreover, ECC fly ash repair layer successfully recovered the structural strength of the cracked NC panel. This can be explained by multiple micro-cracking behavior of ECC along with strain hardening and high tensile strain capacity, which makes ECC an ideal choice for repairing $\mathrm{RC}$ structures including water tanks in which superior cracking and leakage behavior is of prime importance. Use of fly ash F as supplementary cementitious material contributes to the multiple micro-cracking behavior of ECC, superior leakage behavior and its high self-healing ability.

Superior leakage behavior of the NC panels repaired with ECC slag and ECC fly ash can be attributed to the formation of micro-cracks in ECC layer. Therefore, water should penetrate through the full depth of micro-cracks in ECC layer, passing through the bond between the ECC 
layer and NC panel, and travel the full depth of the NC panel to leak from the bottom face of the $\mathrm{NC}$ panel. This mechanism potentially reduced the leakage rate of the NC panels repaired with ECC.

NC panel repaired with GFRP laminate was loaded in tension up to $400 \mathrm{kN}$ and the test was stopped at this level of loading due to exceeding the yielding strain of the rebar and safety reasons. In spite of existence and widening the crack up to $0.78 \mathrm{~mm}$ at $400 \mathrm{kN}$, no failure was observed in GFRP laminate due to its high tensile strength and consequently no leakage was observed in NC panel. Moreover, GFRP laminates showed a significant restraining effect on crack width of NC panel especially at high levels of loading. GFRP laminate successfully improved performance of $\mathrm{NC}$ panel in terms of strength, crack width, and leakage behavior, which indicates effectiveness of the GFRP laminates in waterproofing and recovering the structural strength of the damaged RC members exposed to water pressure and monotonic direct tensile loading such water tanks.

By applying the direct tensile loading on the NC panel repaired with high-strength epoxy injection, failure of epoxy at crack location occurred at tensile load level of $256 \mathrm{kN}$, along with a large crack as wide as $0.65-0.7 \mathrm{~mm}$, which was immediately followed by a high leakage rate. Repairing the mid-span crack of the NC panel with high-pressure injection of the high-strength epoxy significantly improved the tensile load bearing capacity of the panel. Up to the loading stage of $256 \mathrm{kN}$, no failure was observed in epoxy at crack location and epoxy was still capable of carrying the tensile load and preventing crack width growth and leakage initiation. This can be attributed to the high tensile strength and bond strength several times stronger than concrete. Due to brittle nature of the epoxy, failure of the high-strength epoxy at high levels of loading resulted in a substantial increase of the crack width (up to $0.65-0.7 \mathrm{~mm}$ ), followed by a high rate of leakage at mid-span crack. High rate of leakage after failure of the epoxy can be explained by the intimate 
relationship between the crack width and leakage rate. Epoxy injection is known as a local repair solution for cracked RC structures. However, cracks may occur at other locations under the same level of tensile loading, which requires repeating the repair program with epoxy injection. This may affect the efficiency of the epoxy injection solution for repair of RC structures especially in case of liquid containing structures, in which leakage prevention is of prime importance.

Table 4-13: Summary of the performance of different repair materials

\begin{tabular}{|c|c|c|c|c|c|}
\hline Specimen & $\begin{array}{l}\text { Load at leakage } \\
\text { initiation }(\mathrm{kN})\end{array}$ & \multicolumn{2}{|c|}{ Crack width (mm) } & $\begin{array}{l}\text { Water pressure } \\
\quad(\mathrm{psi})\end{array}$ & $\begin{array}{c}\text { Leakage rate } \\
(\mathrm{ml} / \mathrm{hr})\end{array}$ \\
\hline \multirow{4}{*}{ NC (average) } & \multirow{4}{*}{$114-127$} & \multirow{4}{*}{\multicolumn{2}{|c|}{$0.3-0.4$}} & 4 & 4418 \\
\hline & & & & 8 & 9298 \\
\hline & & & & 12 & 12604 \\
\hline & & & & 16 & 15183 \\
\hline \multirow{4}{*}{$\begin{array}{l}\text { NC1- ECC slag } \\
\text { repair }\end{array}$} & \multirow{4}{*}{139} & \multirow{4}{*}{$\begin{array}{l}\text { ECC } \\
0.15\end{array}$} & \multirow{4}{*}{$\begin{array}{l}\mathrm{NC} \\
0.42\end{array}$} & 4 & 166 \\
\hline & & & & 8 & 369 \\
\hline & & & & 12 & 447 \\
\hline & & & & 16 & 517 \\
\hline \multirow{4}{*}{ NC2- FRP repair } & \multirow{4}{*}{$\mathrm{NA}^{*}$} & \multirow{4}{*}{\multicolumn{2}{|c|}{0.78}} & 4 & No leakage \\
\hline & & & & 8 & No leakage \\
\hline & & & & 12 & No leakage \\
\hline & & & & 16 & No leakage \\
\hline \multirow{4}{*}{$\begin{array}{l}\text { NC3- ECC fly ash } \\
\text { repair }\end{array}$} & \multirow{4}{*}{125} & \multirow{4}{*}{$\begin{array}{c}\text { ECC } \\
0.05-0.1\end{array}$} & \multirow{4}{*}{$\begin{array}{l}\mathrm{NC} \\
0.37\end{array}$} & 4 & 67 \\
\hline & & & & 8 & 145 \\
\hline & & & & 12 & 179 \\
\hline & & & & 16 & 215 \\
\hline \multirow{4}{*}{$\begin{array}{l}\text { NC4- Epoxy } \\
\text { Injection repair }\end{array}$} & \multirow{4}{*}{256} & \multirow{4}{*}{\multicolumn{2}{|c|}{$0.65-0.7$}} & 4 & 7864 \\
\hline & & & & 8 & 16354 \\
\hline & & & & 12 & 23155 \\
\hline & & & & 16 & 31357 \\
\hline
\end{tabular}

* Test stopped at $400 \mathrm{kN}$ due to rebar yielding, no failure in GFRP and no leakage was observed.

Comparison of the performance of the repair materials suggests that GFRP laminate exhibited the highest tensile load capacity and prevented the leakage along with restraining the crack width in 
NC panel. High-strength epoxy injection showed high tensile and bond strength, while the brittle nature of epoxy resulted in substantial increase in crack width and leakage rate of NC panel. Moreover, $50 \mathrm{~mm}$ ECC repair layer significantly improved the cracking and leakage behavior of the NC panel and recovered tensile strength of NC panel. Use of fly ash as a partial replacement of cement contributed to the superior cracking and leakage behavior of the ECC, while using slag in ECC resulted in higher tensile capacity but larger crack width and higher leakage rate compared to ECC fly ash.

\section{4-4 Comparison of the self-healing behavior before and after repair using ECC}

Figure 4-33 summarizes and compares the self-healing behavior of the NC panels before and after repair using ECC slag and ECC fly ash. Self-healing behavior of the panels was investigated through measuring the leakage rate of the panel under sustained tensile loading and constant water pressure of 7 psi. As the time prolonged, tension induced crack started to heal and resulted in reducing the leakage rate. $\mathrm{NC}$ panel experienced a sharp drop in the leakage rate during the first 8 hours. Leakage rate of the NC panel before repair decreased from $7867 \mathrm{ml} / \mathrm{hr}$ to $4983 \mathrm{ml} / \mathrm{hr}$ by 37\% after 4 days (as shown on secondary axes in Figure 4-33). However, no significant change in the leakage rate occurred after 35 hours. This can be explained by the fact that there are more available un-reacted cementitious materials during the first few hours to hydrate and block the water pathway through the crack by self-healing products. Therefore, self-healing rate decays over time.

The leakage rate of the NC panel repaired with ECC slag reduced by 68\% from $338 \mathrm{ml} / \mathrm{hr}$ to 107 $\mathrm{ml} / \mathrm{hr}$ during the first 35 hours. A sharp drop was observed in leakage rate during the first 10 hours in which the leakage rate decreased by $41 \%$ from $338 \mathrm{ml} / \mathrm{hr}$ to $199 \mathrm{ml} / \mathrm{hr}$. The rate of leakage 
reduction subsided as the time prolonged which is due to reduction in self-healing capacity of ECC repair layer and NC panel over time. This can be explained by the fact that there are less available cementitious materials over time to participate in self-healing of the concrete panels. Leakage rate of the ECC slag repaired panel reduced to zero after 13 days of monitoring the leakage rate which indicates the water flow with pressure of 7 psi was completely blocked by the self-healing products. Blockage of the water flow could occur either in the ECC repair layer or in the interface between ECC repair layer and NC panel.

The leakage rate of the NC panel repaired with ECC fly ash reduced by $75 \%$ from $138 \mathrm{ml} / \mathrm{hr}$ to 35 $\mathrm{ml} / \mathrm{hr}$ during the first 35 hours. A sharp drop was observed in leakage rate during the first 8 hours in which the leakage rate decreased by $50 \%$ from $138 \mathrm{ml} / \mathrm{hr}$ to $68 \mathrm{ml} / \mathrm{hr}$. The rate of leakage reduction dwindled as the time prolonged which is due to reduction in self-healing capacity of ECC repair layer and NC panel over time. This may attribute to less available cementitious materials over time to take part in self-healing of the concrete panels. Leakage rate in the NC panel repaired with ECC fly ash reduced to zero after 7 days of monitoring the leakage rate which indicates the water flow with pressure of 7 psi was completely blocked by the self-healing products. Blockage of the water flow could occur either in the ECC repair layer or in the interface between ECC repair layer and NC panel. 


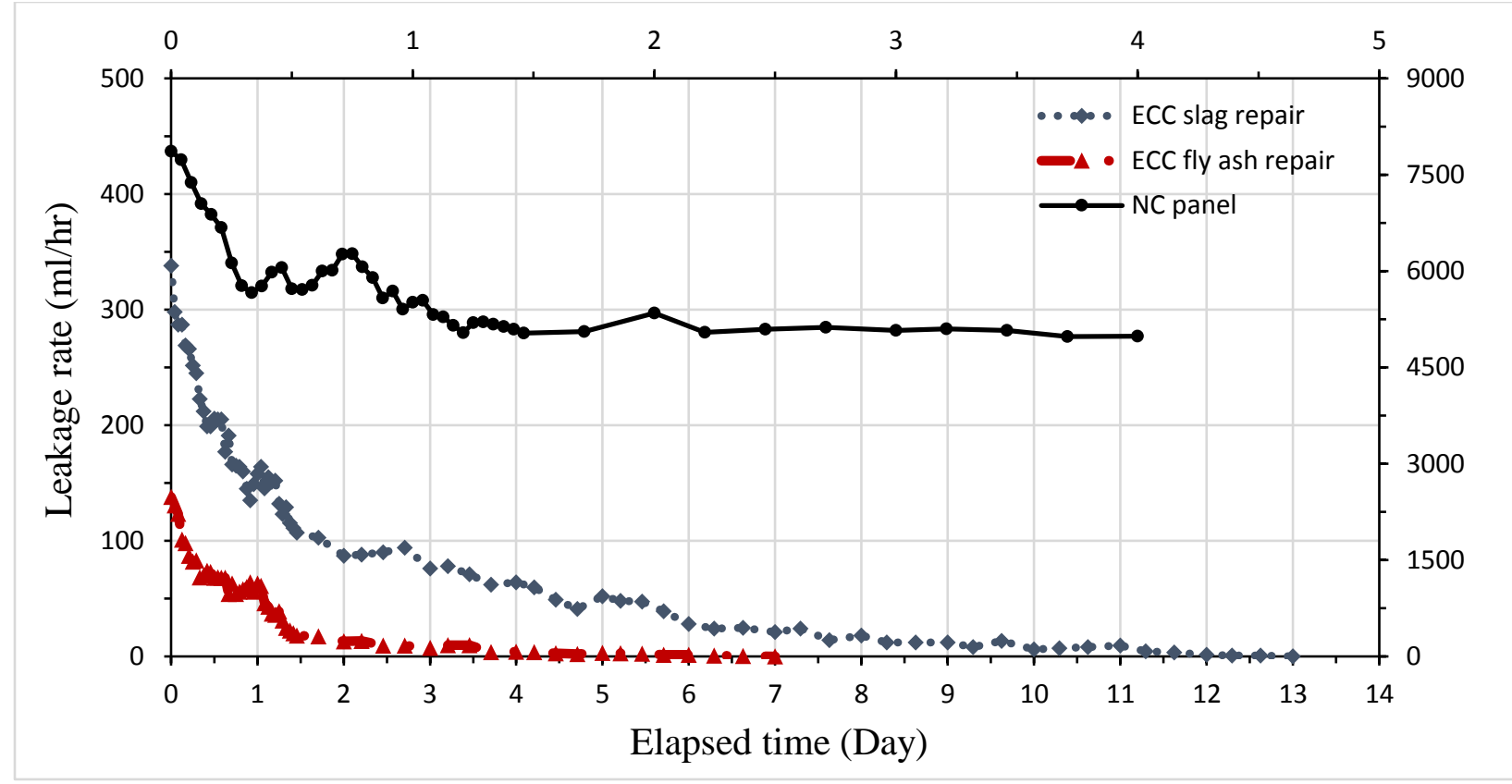

Figure 4-33: Comparison of the self-healing behavior of the NC panel before and after repair using ECC slag and ECC fly ash

Comparison between self-healing behavior of the NC panel and ECC slag and ECC fly ash repaired panels reveals superior self-healing ability of the ECC compared to NC panel. Repair of NC panel with $50 \mathrm{~mm}$ thick layer of ECC significantly improved self-healing behavior of the panel. High self-healing potential of the ECC can be attributed to the availability of high volumes of unreacted cementitious materials as well as its multiple micro-cracking behavior. Multiple micro-cracking behavior by developing cracks as small as $0.1 \mathrm{~mm}$ in ECC results in an initial leakage rate significantly lower than NC panel as well as higher possibility of sealing the crack by self-healing products resulted from hydration of unreacted cementitious materials. Use of fly ash in ECC as supplementary cementitious material resulted in smaller crack width along with higher self-healing capacity of the ECC fly ash repair layer compared to the ECC slag repair layer. 


\section{4-5 Cost analysis}

$\mathrm{RC}$ tanks are constructed to restore water, liquid petroleum, and other liquids. Design of RC tanks are generally performed based on placement condition and shape of the tank. Tanks are usually made in different shapes including circular, rectangular, spherical, intze, and circular tanks with conical bottom. Circular tanks have the lowest surface area in comparison with other shapes for a specific capacity of the tank, resulting in use of less materials for construction of circular tanks. However, fabrication of form-work for circular tanks is more challenging and expensive compared to other tank shapes. In terms of placement condition, RC tanks can be divided to underground tanks, tanks resting on the ground, and overhead tanks. Classification of the tanks based on tank shape and location is shown in Figures 4-34 and 4-35.

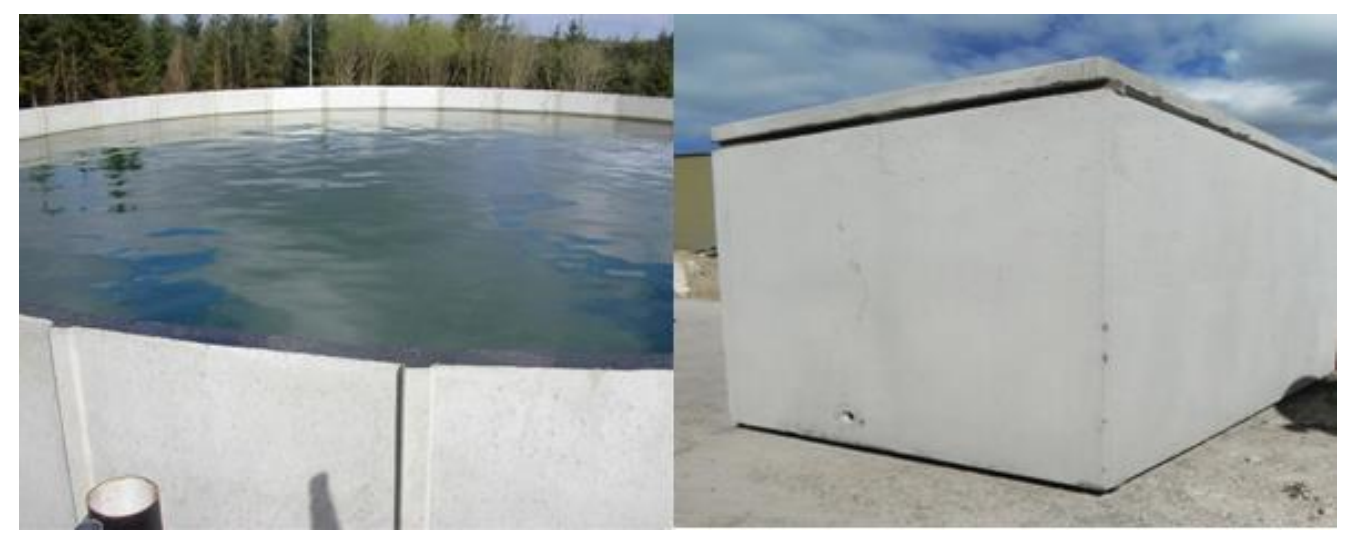

(a)

(b)

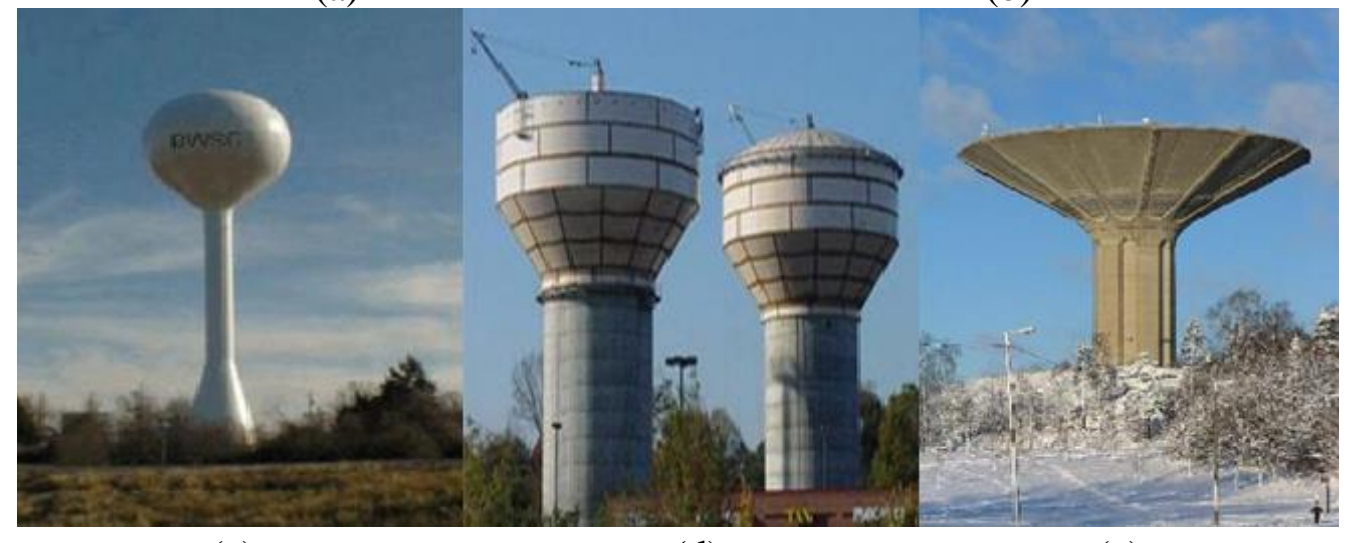

(c)

(d)

(e)

Figure 4-34: Different shapes of the tanks: a) Circular b) Rectangular c) Spherical d) Iznte e) Circular with conical bottom 


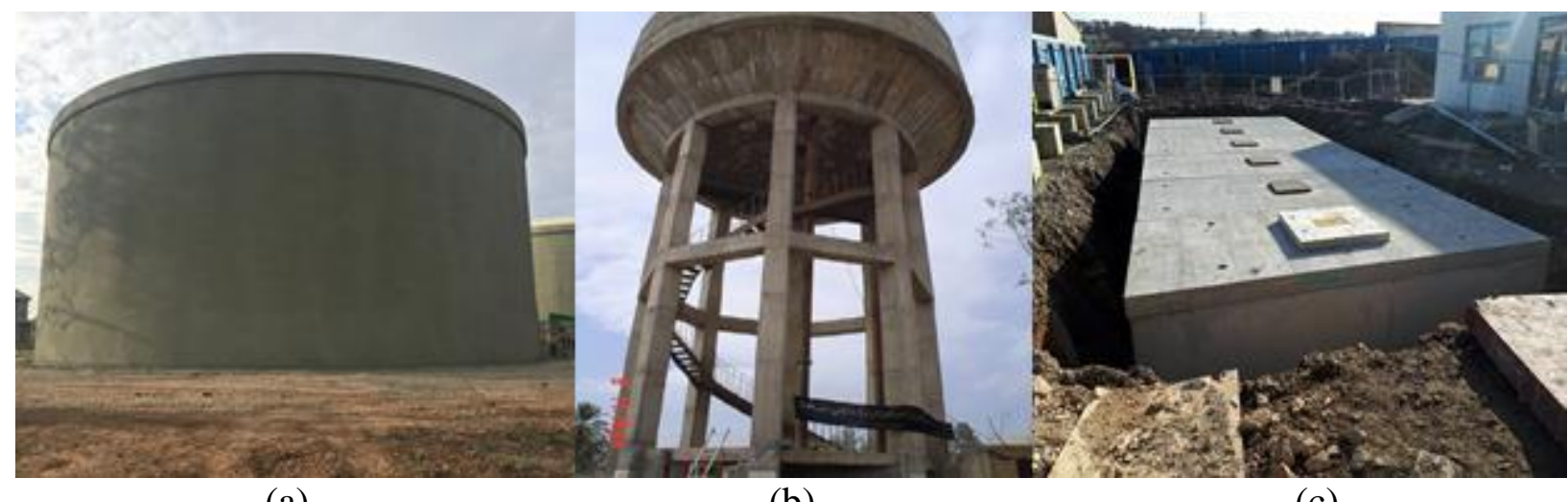

(a)

(b)

(c)

Figure 4-35: Classification of the tanks based on placement condition: a) Resting on the ground b) Elevated c) Underground

Crack control and water tightness are two major factors to be taken into account in design of LCS including water tanks and significantly affect both functionality and durability of these structures. Among different types of cracks, direct tension cracks are most detrimental as they cause leakage initiation in the tank. In this research, circular water tanks resting on the ground with flexible joint at the base are considered and a cost analysis is performed on repair of direct tension cracks in $\mathrm{RC}$ tanks with different capacities by incorporating high-performance materials including ECC fly ash, ECC slag, GFRP laminate, and Carbon Fiber reinforced (CFRP) laminate. The walls of cylindrical tanks are subjected to internal hydro-static water pressure causing hoop tensile stress in the wall which may lead to crack formation and leakage initiation in case of exceeding the tensile strength of concrete. Hydro-static pressure varies from zero at the top to the maximum value at the base in circular tanks with flexible joint at the base. Hoop tension force resulted from hydro-static pressure is calculated for the tanks with various capacities and then the required quantity of each material for repairing the tanks are computed. Next, the total cost of each repair technique is calculated and compared to each other. A wide range of tank capacities are considered to include residential, commercial, and industrial tanks. Residential tanks with capacities ranging from 10500 to 500,000 Liter as well as commercial and industrial tank sizes up to 4,000,000 Liter 
are considered in the cost analysis in the cost analysis. A limited range of $H^{2} / D t$ parameter (4.0 to 4.5) is selected in order to minimize the effect of depth to diameter ratio in the cost analysis. Material properties of GFRP laminate, ECC slag, and ECC fly ash were discussed and presented in Tables 3-6 and 3-7. Furthermore, CFRP laminate has the tensile strength of $1355 \mathrm{MPa}$, tensile modulus of $115700 \mathrm{MPa}$. nominal cured laminate thickness of $1.3 \mathrm{~mm}$, and tensile elongation of $0.95 \%$. It is assumed that the repair program includes applying the ECC or FRP laminate to the full height of the tank. Moreover, the minimum practical thickness of ECC repair layer is considered to be $20 \mathrm{~mm}$ increased with increments of $5 \mathrm{~mm}$. It is worth-mentioning that the prices include materials, form-work, surface preparation, labor, and other miscellaneous expenses. The results of the calculation of hoop tension force, required quantity of repair materials and total cost for each repair technique for different tank capacities are presented in Tables 4-14 to 4-17. It can be observed that increasing the tank capacity resulted in higher water pressure and consequently higher hoop tension force in the wall. Higher levels of hoop tension force also lead to higher quantity of ECC and FRP laminates required for repairing the tank and higher cost of repair. To illustrate, by increasing the tank capacity from 10500 Liter to 4,000,000 Liter, hoop tension force increased from $18.1 \mathrm{kN}$ to $894.1 \mathrm{kN}$ per meter width of the wall. Moreover, the required quantity of ECC fly ash, ECC slag, GFRP laminate, and CFRP laminate enhanced from 0.26 to $77.5 \mathrm{~m}^{3}$, 0.26 to $71.6 \mathrm{~m}^{3}, 13.4$ to $1433 \mathrm{~m}^{2}$, and 13.4 to $717 \mathrm{~m}^{2}$, respectively, by increasing the tank capacity from 10500 Liter to 4.000.000 Liter. Furthermore, the total repair cost of ECC fly ash, ECC slag, GFRP laminate, and CFRP laminate increased from 2080 to 310,069 CAD, 2106 to 292,373 CAD, 780 to $83,642 \mathrm{CAD}$, and 3953 to $212,262 \mathrm{CAD}$, respectively, by enhancing the tank capacity from 10500 Liter to 4,000,000 Liter. 
Table 4-14: Calculation of repair cost for tank capacities between 10500 Liter and 65000 Liter

\begin{tabular}{|c|c|c|c|c|c|c|c|}
\hline \multicolumn{2}{|c|}{ Capacity (Liter) } & 10500 & 15500 & 22500 & 30000 & 50000 & 65000 \\
\hline \multicolumn{2}{|c|}{ Clear height $(\mathrm{mm})$} & 1200 & 1250 & 1400 & 1850 & 1950 & 2100 \\
\hline \multicolumn{2}{|c|}{ Internal diameter $(\mathrm{mm})$} & 3450 & 3900 & 4550 & 5050 & 6350 & 6800 \\
\hline \multicolumn{2}{|c|}{ Depth of water (mm) } & 1050 & 1100 & 1250 & 1650 & 1750 & 1900 \\
\hline \multicolumn{2}{|c|}{ Thickness (mm) } & 100 & 100 & 100 & 150 & 150 & 150 \\
\hline \multicolumn{2}{|c|}{$H^{2} / D t$} & 4.2 & 4.0 & 4.3 & 4.5 & 4.0 & 4.3 \\
\hline \multicolumn{2}{|c|}{ Max hoop tension (kN per unit width) } & 18.1 & 21.5 & 28.4 & 41.7 & 55.6 & 64.6 \\
\hline \multirow{4}{*}{ ECC fly ash } & Thickness (mm) & 20 & 20 & 20 & 20 & 20 & 20 \\
\hline & Price per $m^{3}$ & 8000 & 8000 & 8000 & 8000 & 8000 & 8000 \\
\hline & Volume $\left(\mathrm{m}^{3}\right)$ & 0.260 & 0.306 & 0.400 & 0.587 & 0.778 & 0.897 \\
\hline & Total price $(\mathrm{CAD})$ & 2080 & 2449 & 3200 & 4694 & 6221 & 7174 \\
\hline \multirow{4}{*}{ ECC slag } & Thickness (mm) & 20 & 20 & 20 & 20 & 20 & 20 \\
\hline & Price per $m^{3}$ & 8100 & 8100 & 8100 & 8100 & 8100 & 8100 \\
\hline & Volume $\left(m^{3}\right)$ & 0.260 & 0.306 & 0.400 & 0.587 & 0.778 & 0.897 \\
\hline & Total price $(\mathrm{CAD})$ & 2106 & 2480 & 3240 & 4752 & 6299 & 7264 \\
\hline \multirow{4}{*}{ GFRP laminate } & Number of layers & 1 & 1 & 1 & 1 & 1 & 1 \\
\hline & Price per $m^{2}$ & 58 & 58 & 58 & 58 & 58 & 58 \\
\hline & Area $\left(m^{2}\right)$ & 13.4 & 15.7 & 24.7 & 34.6 & 45.4 & 51.9 \\
\hline & Total price (CAD) & 780 & 915 & 1442 & 2022 & 2653 & 3028 \\
\hline \multirow{4}{*}{ CFRP laminate } & Number of layers & 1 & 1 & 1 & 1 & 1 & 1 \\
\hline & Price per $m^{2}$ & 296 & 296 & 296 & 296 & 296 & 296 \\
\hline & Area $\left(m^{2}\right)$ & 13.4 & 15.7 & 24.7 & 34.6 & 45.4 & 51.9 \\
\hline & Total price (CAD) & 3953 & 4640 & 7311 & 10252 & 13448 & 15350 \\
\hline
\end{tabular}


Table 4-15: Calculation of repair cost for tank capacities between 107000 Liter and 260000 Liter

\begin{tabular}{|c|c|c|c|c|c|c|c|}
\hline \multicolumn{2}{|c|}{ Capacity (Liter) } & 107000 & 120000 & 150000 & 170000 & 200000 & 260000 \\
\hline \multicolumn{2}{|c|}{ Clear height $(\mathrm{mm})$} & 2250 & 2300 & 2400 & 2500 & 2600 & 2700 \\
\hline \multicolumn{2}{|c|}{ Internal diameter (mm) } & 8250 & 8650 & 9450 & 9850 & 10550 & 11750 \\
\hline \multicolumn{2}{|c|}{ Depth of water (mm) } & 2050 & 2100 & 2200 & 2250 & 2350 & 2450 \\
\hline \multicolumn{2}{|c|}{ Thickness (mm) } & 150 & 150 & 150 & 150 & 150 & 150 \\
\hline \multicolumn{2}{|c|}{$H^{2} / D t$} & 4.1 & 4.1 & 4.1 & 4.2 & 4.3 & 4.1 \\
\hline \multicolumn{2}{|c|}{ Max hoop tension (kN per unit width) } & 84.6 & 90.8 & 104.0 & 110.8 & 124.0 & 143.9 \\
\hline \multirow{4}{*}{ ECC fly ash } & Thickness (mm) & 20 & 20 & 20 & 20 & 20 & 25 \\
\hline & Price per $m^{3}$ & 7000 & 7000 & 7000 & 7000 & 7000 & 7000 \\
\hline & Volume $\left(m^{3}\right)$ & 1.166 & 1.249 & 1.424 & 1.546 & 1.723 & 2.490 \\
\hline & Total price (CAD) & 8160 & 8746 & 9970 & 10825 & 12058 & 17433 \\
\hline \multirow{4}{*}{ ECC slag } & Thickness (mm) & 20 & 20 & 20 & 20 & 20 & 25 \\
\hline & Price per $m^{3}$ & 7100 & 7100 & 7100 & 7100 & 7100 & 7100 \\
\hline & Volume $\left(\mathrm{m}^{3}\right)$ & 1.166 & 1.249 & 1.424 & 1.546 & 1.723 & 2.490 \\
\hline & Total price (CAD) & 8277 & 8871 & 10113 & 10980 & 12230 & 17682 \\
\hline \multirow{4}{*}{ GFRP laminate } & Number of layers & 1 & 1 & 1 & 1 & 1 & 1 \\
\hline & Price per $m^{2}$ & 58 & 58 & 58 & 58 & 58 & 58 \\
\hline & Area $\left(m^{2}\right)$ & 66.7 & 71.3 & 80.8 & 87.4 & 106.8 & 122.6 \\
\hline & Total price (CAD) & 3895 & 4162 & 4719 & 5099 & 6233 & 7154 \\
\hline \multirow{4}{*}{ CFRP laminate } & Number of layers & 1 & 1 & 1 & 1 & 1 & 1 \\
\hline & Price per $m^{2}$ & 296 & 296 & 296 & 296 & 296 & 296 \\
\hline & $\operatorname{Area}\left(m^{2}\right)$ & 66.7 & 71.3 & 80.8 & 87.4 & 106.8 & 122.6 \\
\hline & Total price (CAD) & 19746 & 21100 & 23920 & 25847 & 31598 & 36267 \\
\hline
\end{tabular}


Table 4-16: Calculation of repair cost for tank capacities between 315000 Liter and 850000 Liter

\begin{tabular}{|c|c|c|c|c|c|c|c|}
\hline \multicolumn{2}{|c|}{ Capacity (Liter) } & 315000 & 400000 & 500000 & 650000 & 750000 & 850000 \\
\hline \multicolumn{2}{|c|}{ Clear height $(\mathrm{mm})$} & 2800 & 2900 & 3000 & 3600 & 3750 & 3850 \\
\hline \multicolumn{2}{|c|}{ Internal diameter (mm) } & 12700 & 14000 & 15100 & 15600 & 16400 & 17250 \\
\hline \multicolumn{2}{|c|}{ Depth of water (mm) } & 2550 & 2650 & 2750 & 3350 & 3500 & 3600 \\
\hline \multicolumn{2}{|c|}{ Thickness (mm) } & 150 & 150 & 150 & 200 & 200 & 200 \\
\hline \multicolumn{2}{|c|}{$H^{2} / D t$} & 4.1 & 4.0 & 4.0 & 4.2 & 4.3 & 4.3 \\
\hline \multicolumn{2}{|c|}{ Max hoop tension (kN per unit width) } & 161.9 & 185.5 & 207.6 & 261.3 & 287.0 & 310.5 \\
\hline \multirow{4}{*}{ ECC fly ash } & Thickness (mm) & 25 & 30 & 35 & 45 & 45 & 50 \\
\hline & Price per $m^{3}$ & 7000 & 7000 & 7000 & 6000 & 6000 & 6000 \\
\hline & Volume $\left(m^{3}\right)$ & 2.791 & 3.825 & 4.978 & 7.935 & 8.690 & 10.427 \\
\hline & Total price (CAD) & 19540 & 26772 & 34849 & 47612 & 52140 & 62561 \\
\hline \multirow{4}{*}{ ECC slag } & Thickness (mm) & 25 & 25 & 30 & 40 & 45 & 45 \\
\hline & Price per $m^{3}$ & 7100 & 7100 & 7100 & 6100 & 6100 & 6100 \\
\hline & Volume $\left(\mathrm{m}^{3}\right)$ & 2.791 & 3.187 & 4.267 & 7.054 & 8.690 & 9.384 \\
\hline & Total price (CAD) & 19819 & 22628 & 30298 & 43028 & 53009 & 57243 \\
\hline \multirow{4}{*}{ GFRP laminate } & Number of layers & 1 & 1 & 1 & 1 & 1 & 1 \\
\hline & Price per $m^{2}$ & 58 & 58 & 58 & 58 & 58 & 58 \\
\hline & Area $\left(m^{2}\right)$ & 136.4 & 154.7 & 171.6 & 206.8 & 225.1 & 258.4 \\
\hline & Total price (CAD) & 7964 & 9032 & 10016 & 12072 & 13142 & 15086 \\
\hline \multirow{4}{*}{ CFRP laminate } & Number of layers & 1 & 1 & 1 & 1 & 1 & 1 \\
\hline & Price per $m^{2}$ & 296 & 296 & 296 & 296 & 296 & 296 \\
\hline & $\operatorname{Area}\left(m^{2}\right)$ & 136.4 & 154.7 & 171.6 & 206.8 & 225.1 & 258.4 \\
\hline & Total price $(\mathrm{CAD})$ & 40368 & 45785 & 50774 & 61196 & 66617 & 76472 \\
\hline
\end{tabular}


Table 4-17: Calculation of repair cost for tank capacities between 950000 Liter and 4000000 Liter

\begin{tabular}{|c|c|c|c|c|c|c|}
\hline \multicolumn{2}{|c|}{ Capacity (Liter) } & 950000 & 1000000 & 2000000 & 3000000 & 4000000 \\
\hline \multicolumn{2}{|c|}{ Clear height $(\mathrm{mm})$} & 3950 & 4000 & 4500 & 5500 & 6000 \\
\hline \multicolumn{2}{|c|}{ Internal diameter $(\mathrm{mm})$} & 18000 & 18350 & 24350 & 28250 & 31650 \\
\hline \multicolumn{2}{|c|}{ Depth of water (mm) } & 3700 & 3750 & 4250 & 5200 & 5650 \\
\hline \multicolumn{2}{|c|}{ Thickness (mm) } & 200 & 200 & 200 & 250 & 250 \\
\hline \multicolumn{2}{|c|}{$H^{2} / D t$} & 4.3 & 4.4 & 4.2 & 4.3 & 4.5 \\
\hline \multicolumn{2}{|c|}{ Max hoop tension (kN per unit width) } & 333.0 & 344.1 & 517.4 & 734.5 & 894.1 \\
\hline \multirow{4}{*}{ ECC fly ash } & Thickness (mm) & 55 & 55 & 85 & 110 & 130 \\
\hline & Price per $m^{3}$ & 6000 & 6000 & 5000 & 4000 & 4000 \\
\hline & Volume $\left(m^{3}\right)$ & 12.279 & 12.676 & 29.246 & 53.667 & 77.517 \\
\hline & Total price (CAD) & 73674 & 76057 & 146228 & 214666 & 310069 \\
\hline \multirow{4}{*}{ ECC slag } & Thickness (mm) & 50 & 50 & 75 & 100 & 120 \\
\hline & Price per $m^{3}$ & 6100 & 6100 & 5100 & 4100 & 4100 \\
\hline & Volume $\left(m^{3}\right)$ & 11.163 & 11.524 & 25.805 & 48.788 & 71.554 \\
\hline & Total price (CAD) & 68092 & 70295 & 131605 & 200030 & 293373 \\
\hline \multirow{4}{*}{ GFRP laminate } & Number of layers & 1 & 1 & 2 & 2 & 2 \\
\hline & Price per $m^{2}$ & 58 & 58 & 58 & 58 & 58 \\
\hline & Area $\left(m^{2}\right)$ & 275.3 & 283.5 & 827.1 & 1190.3 & 1432.9 \\
\hline & Total price (CAD) & 16070 & 16551 & 48281 & 69481 & 83642 \\
\hline \multirow{4}{*}{ CFRP laminate } & Number of layers & 1 & 1 & 1 & 1 & 1 \\
\hline & Price per $m^{2}$ & 296 & 296 & 296 & 296 & 296 \\
\hline & Area $\left(m^{2}\right)$ & 275.3 & 283.5 & 414.2 & 596.0 & 717.3 \\
\hline & Total price (CAD) & 81463 & 83897 & 122570 & 176349 & 212262 \\
\hline
\end{tabular}


Figures 4.36 illustrates the total cost of repairing the commercial and industrial RC tanks with capacity up to 4,000,000 Liter. It can be observed that the repair cost of all materials increased continuously by enlarging the tanks. GFRP laminate exhibited the lowest price among all repair materials in repairing RC tanks with capacities up to 4,000,000 Liter. ECC fly ash and ECC slag showed almost the same cost of repair due to similar quantity required for the repair and similar mix design. For the tank capacities up to almost 1,500,000 Liter, repairing the tank using CFRP laminate showed the highest price, while for the tank capacity greater than 1,500,000 Liter, ECC overlay system exhibited the highest price. The total price of repairing a RC tank with capacity of 1,000,000 using ECC fly ash, ECC slag, GFRP laminate, and CFRP laminate was 76057, 70295, 16551 , and $83897 \mathrm{CAD}$, respectively. However, by enlarging the tank to a capacity of 4,000,000 Liter, the total cost of repair by incorporating ECC fly ash, ECC slag, GFRP laminate, and CFRP laminate were $310069,293373,83642$, and $21262 \mathrm{CAD}$, respectively.

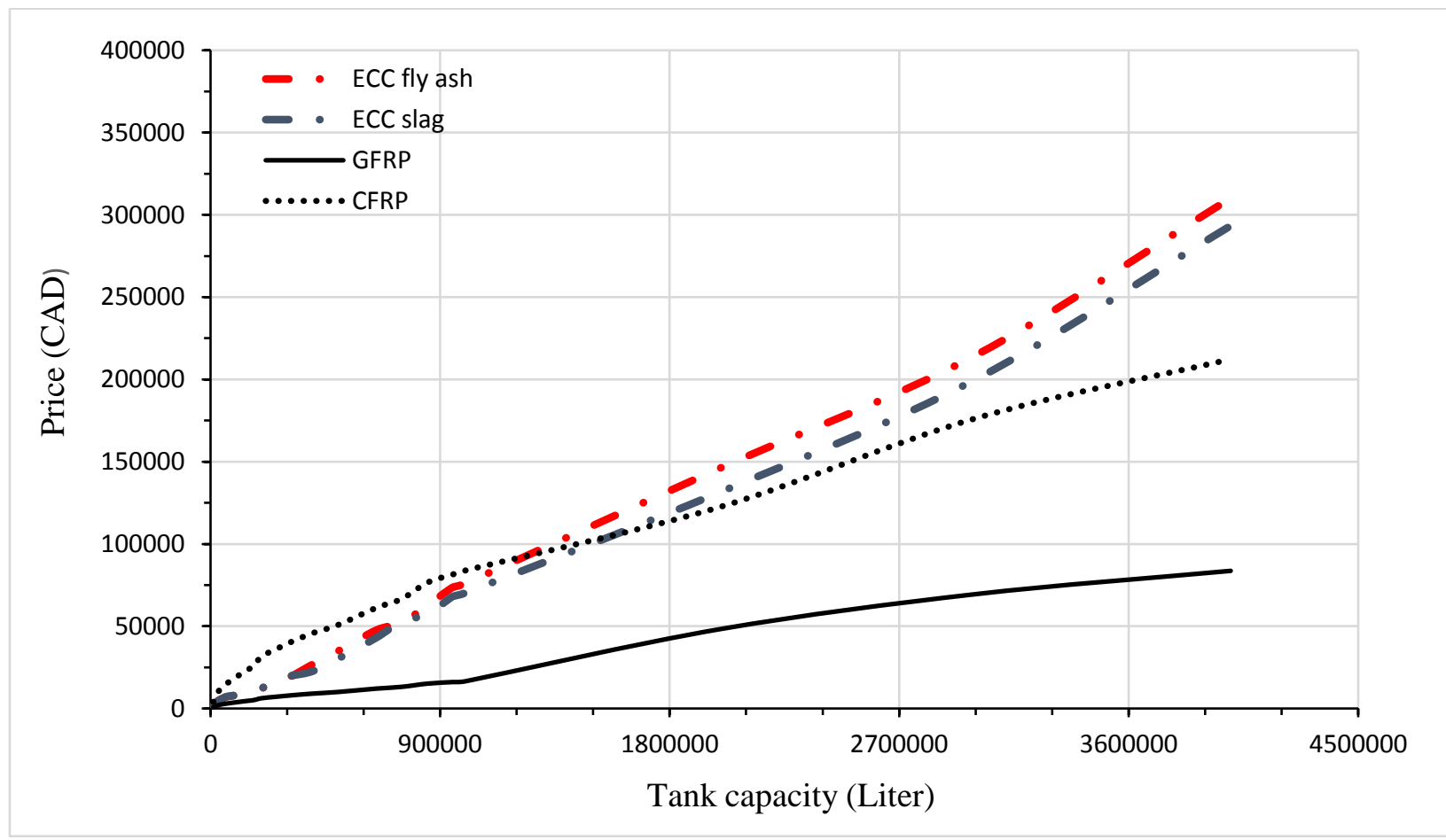

Figure 4-36: Total cost of repair for commercial and industrial tanks (up to 4,000,000 Liter) 
Figure 4-37 shows the repair cost of residential RC tanks with capacity up to 500,000 Liter. It can be observed that ECC fly ash and ECC slag showed almost the same price in repairing the RC tanks. Moreover, repairing the tank with CFRP laminate exhibited the highest price, while GFRP laminate showed the lowest price in repairing residential tanks with capacity up to 500,000 Liter compared to other repair materials. For example, the total price of repairing a RC tank with capacity of 500,000 Liter using ECC fly ash, ECC slag, GFRP laminate, and CFRP laminate were 34849, 30298, 10016, and 50774 CAD, respectively.

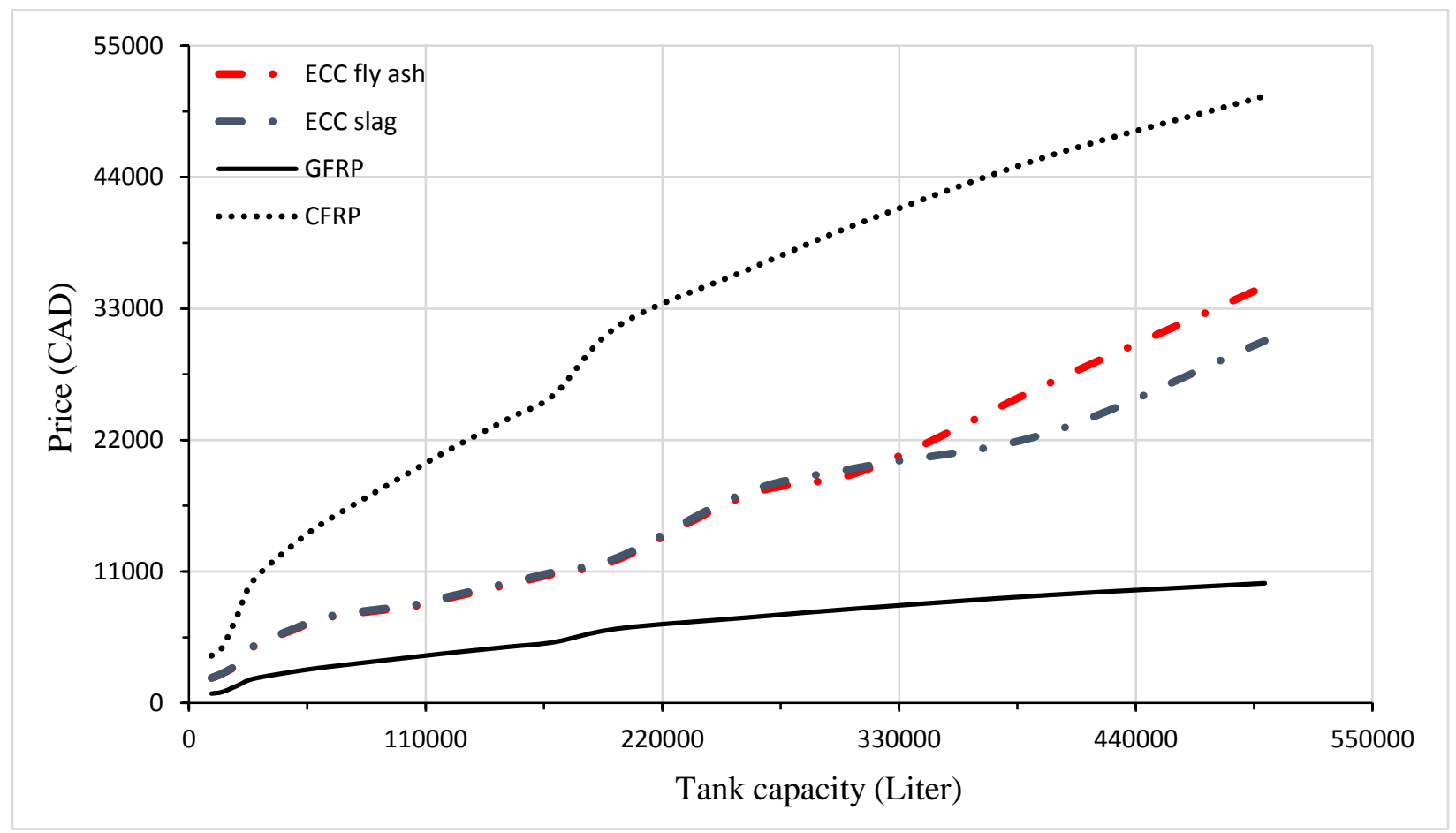

Figure 4-37: Total cost of repair for residential tanks (up to 500,000 Liter) 


\section{4-6 Conclusions}

This chapter describes the performance of ECC slag, ECC fly ash, high-strength epoxy, and GFRP laminates in repairing the NC panel exposed to direct tensile loading and hydrostatic water pressure based on experimental results. Load versus horizontal displacement responses, strain development in the steel rebar, crack development and crack width, leakage rate under various water pressures were analyzed to evaluate the performance of each repair material in terms of tensile load capacity, cracking and leakage behavior. Self-healing behavior of the concrete materials were also investigated. The analysis revealed the effectiveness of the GFRP laminate in waterproofing and repair of damaged structures under monotonic tensile loading. The results pointed out to the superior cracking and leakage behavior, high self-healing ability, and capability of ECC in recovering the tensile strength of the damaged structures, making it an appropriate choice for repair of RC members specially water tanks. The comparison of the experimental results also suggested the applicability of epoxy injection for local repair of the cracked RC members. Based on the results of cost analysis, GFRP laminate was found to be the most economical method of repairing $\mathrm{RC}$ water tanks. 


\section{Chapter Five: Conclusions}

\section{5-1 General}

The thesis studied the repair and self-healing of tension induced cracks in reinforced concrete (RC) panels under direct tensile loading by incorporating high-strength epoxy injection, Glass Fiber Reinforced Polymer (GFRP) laminates, Engineered Cementitious Composite (ECC) with slag, and ECC with fly ash. Two types of supplementary cementitious materials (SCMs), slag and fly ash, were used as a partial replacement of the cement in ECC mix design to produce ECC slag and ECC fly ash, respectively. A total number of 4 full-scale RC panels made with normal concrete (NC) were fabricated and tested under monotonic direct tensile loading and hydrostatic water pressure. Cracked RC panels were repaired with above-mentioned high-performance materials. Load at leakage initiation, crack development and crack width, leakage rate under various water pressures, load versus horizontal displacement responses, and strain development in the steel rebar were analyzed to evaluate the performance of RC panels before repair as well as efficiency of each repair material in terms of tensile load capacity, cracking and leakage behavior. Short-term and long-term self-healing behavior of the RC panels before and after repair using ECC slag and ECC fly ash was also investigated. A cost analysis was also performed to evaluate the cost-effectiveness of different high-performance materials in repair of RC water tanks.

Each RC panel in this experimental program represents the wall segment of a water tank which is under internal hydrostatic pressure of water. Test setup simulates repairing the cracked wall segment of the water tank with high-strength epoxy injection, ECC slag and ECC fly ash repair 
layer, GFRP laminates. Applying the repair on top surface of RC panels represents repairing the inside surface of the water tank, which is in contact with hydrostatic water pressure.

\section{5-2 Conclusions}

The following conclusions are obtained based on this experimental program:

- NC panels before repair showed similar cracking and leakage behavior under direct tensile loading and hydrostatic water pressure. Sudden cracking of NC panel along with simultaneous strength failure and leakage initiation indicate how brittle and undesired regular RC panels could be in terms of serviceability performance. Moreover, NC panels showed only a partial self-healing capacity due to development of a large crack width along with low quantity of available unreacted cementitious materials.

- No failure occurred due to tensile rupture and debonding of the GFRP laminate (due to its high tensile strength) and consequently no leakage was observed in GFRP repaired NC panel even at loading levels close to or beyond the yield of reinforcement. GFRP laminate significantly improved performance of $\mathrm{NC}$ panel in terms of strength, and cracking control, indicating the effectiveness of the GFRP laminates in waterproofing and recovering the structural strength of the damaged RC members exposed to water pressure and monotonic direct tensile loading such as water tanks.

- Selecting the appropriate number of GFRP layers is of prime importance. Insufficient number of GFRP layers may result in a brittle failure of the laminate under tensile loading followed by a high leakage rate at crack location, which potentially threatens the efficiency of the repair program especially in case of water tanks. 
- Repairing the mid-span crack of NC panel with high-pressure injection of the high-strength epoxy significantly improved the tensile load at which leakage initiated. This can be attributed to the high tensile and bond strengths of epoxy several times stronger than concrete. However, due to brittle nature of epoxy, failure of the high-strength epoxy at high levels of loading resulted in a substantial increase of the crack width followed by a high rate of leakage at mid-span crack.

- Epoxy injection is known as a local repair solution for cracked RC structures. However, cracks may occur at other locations under the same level of tensile loading, which requires repeating the repair program with epoxy injection. This may affect the efficiency of the epoxy injection solution for repair of RC structures especially in case of LCS, in which leakage prevention is of prime importance.

- ECC fly ash and ECC slag repair layers significantly improved the cracking and leakage behavior along with recovering the structural strength of the cracked NC panel. This can be explained by multiple micro-cracking behavior of ECC along with strain hardening and high tensile strain capacity, making ECC an ideal choice for repairing RC structures including water tanks, in which superior cracking and leakage behavior is of prime importance. Use of fly ash as a partial replacement of cement contributed to the superior cracking and leakage behavior of ECC, while using slag in ECC resulted in higher tensile capacity but larger crack width and higher leakage rate compared to ECC fly ash.

- Superior leakage behavior of NC panels repaired using ECC can be attributed to the formation of micro-cracks in ECC layer. Mechanism of traveling of water through the micro-cracks in ECC layer, interface of ECC and NC panel, and full depth of NC panel potentially reduced the leakage rate of ECC repaired NC panels. 
- Full self-healing of cracks was achieved in ECC repaired NC panels due to large quantity of available unreacted cementitious materials and multiple cracking behavior of ECC.

- The study confirmed the applicability of ECC fly ash in repairing LCS with superior cracking and leakage behavior along with ability to restore the structural strength of the damaged member. Higher thicknesses of ECC repair layer can be used to further improve the tensile strength of the damaged RC member.

- The results of cost analysis suggested GFRP laminate as the most economical technique for repair of RC water tanks compared to ECC fly ash, ECC slag, and CFRP laminate.

\section{5-3 Recommendations for future research}

The following recommendations are suggested for further research studies:

- Investigations are to be conducted on the effect of the ECC repair layer thickness on improving the cracking, leakage, and self-healing behavior as well as structural strength of the damaged $\mathrm{RC}$ members under direct tensile loading and hydrostatic water pressure such as liquid containing structures. Moreover, effect of the level of substrate surface roughness on the performance of ECC overlay system could be an interesting topic for further research.

- Additional experimental investigations would be helpful to study the effectiveness of other types of high-performance concrete such as Ultra High-Performance Concrete (UHPC) in the repair of NC panels under direct tensile loading and hydrostatic water pressure. This will help to assess the applicability of the UHPC in repairing of liquid containing structures. It is expected that high tensile capacity of the UHPC will significantly improve structural strength of the damaged NC panel. However, presence of silica fume in UHPC mix design 
would negatively affect the cracking, leakage behavior of the NC panel repaired with UHPC. Moreover, since self-healing capacity depends on the available unreacted cementitious materials, use of silica fume with potentially high rate of hydration may affect the self-healing ability of the NC panel repaired with UHPC. Effect of incorporating various UHPC repair layer thicknesses can be investigated as well.

- In addition to water tanks, further research on the effectiveness of the high-strength epoxy, GFRP laminates, and ECC in repairing concrete tanks containing chemicals can enlarge the applicability of these high-performance materials in repair of LCS.

- Investigations can be carried out on the application of ECC repair layers on both top and bottom surfaces of the NC panel which simulates repairing both inside and outside of the cracked wall of a water tank. This repair method is expected to improve cracking, leakage, and self-healing behavior as well as structural strength of the cracked wall of the water tank. Moreover, the ECC repair layer outside of the tank would provide a protective layer for the tank against ingress of aggressive agents such as chloride, acid, and sulphate attacks. This may significantly reduce corrosion of the rebars as well as other modes of deteriorations which threatens both durability and functionality of water tanks. 


\section{References}

AASHTO M235-13, (2018). Specification for Epoxy Resin Adhesives, American Association of State Highway Transportation Officials (AASHTO), Washington, DC, USA, 2013

ACI 224R-01, (2001). Control of Cracking in Concrete Structures, American Concrete Institute, Farmington Hills, Mich., 2001, 46 pp.

ACI 224.1R-07, (2007). Causes, Evaluation, and Repair of Cracks in Concrete Structures. American Concrete Institute International.

ACI 350-06, (2006). Code Requirements for Environmental Engineering Concrete Structures, American Concrete Institute.

ACI 440.2R-08, (2008). Guide for the design and construction of externally bonded FRP systems for strengthening concrete structures. Reported by ACI committee 440.

ACI 546R-14, (2014). Guide to Concrete Repair. American Concrete Institute. Reported by ACI Committee 546.

Ahmad, S., Elahi, A., Barbhuiya, S., \& Farooqi, Y. (2013). Repair of cracks in simply supported beams using epoxy injection technique. Materials and Structures/Materiaux et Constructions, 46(9), 1547-1559.

AL-Gemeel, A. N., \& Zhuge, Y. (2018). Experimental investigation of textile reinforced engineered cementitious composite (ECC) for square concrete column confinement. Construction and Building Materials, 174, 594-602.

Allen, H. G. (1971). Stiffness and strength of two glass-fiber reinforced cement laminates. Journal of Composite Materials, 5(2), 194-207.

Anwar, A. M., Hattori, K., Ogata, H., Ashraf, M., \& M. (2009). Engineered Cementitious Composites for Repair of Initially Cracked Concrete Beams. Asian Journal of Applied Sciences.

ASTM C39, (2012). Standard test method for compressive strength of cylindrical concrete specimens., ASTM International, West Conshohocken, PA.

ASTM C78/C78M-18, (2018). Standard test method for flexural strength of concrete (using simple beam with third-point loading). ASTM International, West Conshohocken, PA.

ASTM C496/C496M-17, (2017). Standard Test Method for Splitting Tensile Strength of Cylindrical Concrete Specimens, ASTM International, West Conshohocken, PA.

ASTM Standard C642, (2006). Standard Test Method for Density, Absorption, and Voids in Hardened Concrete. West Conshohocken, PA: ASTM International.

ASTM Standard C666/C666M-03, (2008). Standard Test Method for Resistance of Concrete to Rapid Freezing and Thawing. West Conshohocken, PA: ASTM International. 
ASTM C672/C672M, (2012). Standard test method for resistance of concrete to rapid freezing and thawing. ASTM International, West Conshohocken, PA.

ASTM C881/C881M-15, (2015), Standard specification for epoxy-resin-base bonding systems for concrete. ASTM International, West Conshohocken, PA.

ASTM Standard C1260-94, (1994). Standard Test Method for Potential Alkali Reactivity of Aggregates (Mortar-bar method). West Conshohocken, PA: ASTM International.

ASTM Standard C1585, (2011). Standard Test Method for Measurement of Rate of Absorption of Water by Hydraulic-Cement Concretes. West Conshohocken, PA: ASTM International.

Aveston, J., Cooper, G. A., \& Kelly, A. (1971). Single and multiple fracture. Paper presented at The Properties of Fiber Composites Conference, Guildford, U.K., (pp. 15-24). IPC Science and Technology Press.

Baker, J. W. (2007). Measuring bias in structural response caused by ground motion scaling. Pacific Conference on Earthquake Engineering, (056), 1-6.

Çavdar, A. (2012). A study on the effects of high temperature on mechanical properties of fiber reinforced cementitious composites, Composites: Part B, 43(5) 2452-2463.

Chang, P., Peng, Y. and Hwang, C. (2001). A Design Consideration for Durability of HighPerformance Concrete. Cement and Concrete Composites, 23(4-5), pp. 375-380.

Chu, K. (2014). Axial load behaviour of steel tube columns infilled with various high-performance concretes, Master Thesis, Ryerson University, Toronto, Ontario.

Deng, M., Zhang, Y., \& Li, Q. (2018). Shear strengthening of RC short columns with ECC jacket: Cyclic behavior tests. Engineering Structures, 160(November 2017), 535-545.

Edvardsen, C. (1999). Water permeability and autogenous healing of cracks in concrete. ACI Mater. J. 96.

Federal Highway Administration (FHWA), (1992). Corossion Detection in Reinforced Concrete Bridge Structures. Washington, DC: Project 84.

Fischer, G. and Li, V.C. (2002). Effect of matrix ductility on deformation behavior of steel reinforced ECC flexural members under reversed cyclic loading conditions. ACI Structural J. 99 (6):781-790.

Fischer, G., Li, V.C. (2003). Deformation Behavior of Fiber-Reinforced Polymer Reinforced Engineered Cementitious Composite (ECC) Flexural Members under Reversed Cyclic Loading Conditions. Struct. J. 100, 25-35.

Fischer, G., Wang, S., \& Li, V. C. (2003). Design of engineered cementitious composites for processing and workability requirements. Paper presented at the 7 th International Symposium on Brittle Matrix Composites, Warsaw, Poland, 13-15 October (pp. 29-35).

Giulietti, \& Assumpção. (2019). 済無No Title No Title. Journal of Chemical Information and Modeling, 53(9), 1689-1699. 
Griffin, S., Askarinejad, H., \& Farrant, B. (2017). Evaluation of Epoxy Injection Method for Concrete Crack Repair. International Journal of Structural and Civil Engineering Research, (July), 177-181.

Hag-Elsafi, O., Alampalli, S., \& Kunin, J. (2001). Application of FRP laminates for strengthening of a reinforcedconcrete T-beam bridge structure. Composite Structures, 52(3-4), 453-466.

Herbert, E., Li, V. (2013). Self-Healing of Microcracks in Engineered Cementitious Composites (ECC) Under a Natural Environment. Materials 6, 2831-2845.

Hossain, K.M.A., and Anwar, M.S. (2014). Strength and Deformation Characteristic of ECC Link Slab in Joint-Free Bridge Decks, Istanbul Bridge Conference, August, Istanbul, Turkey.

Hooshmand, A., Kianoush, M. R., Lachemi, M., Siad, H., \& Moslemi, M. (2019). An experimental study on self-healing on tension cracks in ECC and concrete panels, CSCE annual conference, Laval, 2019.

Hou, W., Li, Z. Q., Gao, W. Y., Zheng, P. D., \& Guo, Z. X. (2020). Flexural behavior of RC beams strengthened with BFRP bars-reinforced ECC matrix. Composite Structures, 241(February), 112092.

Hung, C. C., \& Chen, Y. S. (2016). Innovative ECC jacketing for retrofitting shear-deficient RC members. Construction and Building Materials, 111, 408-418.

Hwang, C., Liu, J., Lee, L. and Lin, F. (1996). Densified Mixture Design Algorithm and Early Properties of High-Performance Concrete. Chinese Institute of Civil and Hydraulic Engineering, 8(2), pp. 217-229.

Issa, C. A., \& Debs, P. (2007). Experimental study of epoxy repairing of cracks in concrete. Construction and Building Materials, 21(1), 157-163.

Kan, L.-L., Shi, H.-S., Sakulich, A.R., Li, V.C. (2010). Self-Healing Characterization of Engineered Cementitious Composite Materials. Mater. J. 107, 619-626.

Kanda, T., Saito, T. and Sakata, N. (2003). Tensile and Anti-Spalling Properties of Direct Sprayed ECC. Advanced Concrete Technology, 1(3), pp. 269-282.

Kianoush, M.R., Acarcan, M., Ziari, A. (2008). Behavior of base restrained reinforced concrete walls under volumetric change. Eng. Struct. 30, 1526-1534.

Kim, Y. Y., Lee, B. Y., Bang, J. W., Han, B. C., Feo, L., \& Cho, C. G. (2014). Flexural performance of reinforced concrete beams strengthened with strain-hardening cementitious composite and high strength reinforcing steel bar. Composites Part B: Engineering, 56, 512519.

Kojima, S., Sakat, N., Kanda, T. and Hiraishi, T. (2004). Application of direct sprayed ECC for retrofitting dam structure surface - application for Mitaka-Dam. Concrete Journal 42(5):3539. 
Krenchel, H., \& Stang, H. (1989). Stable microcracking in cementitious materials. In A. M. Brandt, \& J. H. Marshall (Eds.), Brittle Matrix Composites 2, (pp. 20-33). Elsevier, Amsterdam.

Kunieda, M., and Rokugo, K. (2006), "Recent progress on HPFRCC in Japan: Required performance and applications", Journal of Advances in Concrete Technology, 4(1) 19-33.

Lankard, D. R. (1986). Preparation, properties and applications of cement-based composites containing 5-20 percent steel fiber reinforcement. In S. P. Shah, \& A. Skarendahl (Eds.), Steel Fiber Concrete. Elsevier, Amsterdam.

Lepech, M., Li, V.C. (2005a). Water Permeability of Cracked Cementitious Composites, in: Proceedings of ICF11, Paper 4539. CD-ROM.

Lepech, M. and Li, V. (2005b). Durability and Long-term Performance of Engineered Cementitious Composites. Honolulu, Hawaii, In Proceedings of International RILEM Workshop on HPFRCC in Structural Applications, pp. 165-174.

Lepech, M.D. and Li, V. (2006). Long Term Durability Performance of Engineered Cementitious Composites. Journal of Restoration of Buildings and Monuments, 12(2), pp. 119-132.

Lepech, M. D., \& Li, V. C. (2008). Large-scale processing of engineered cementitious composites. ACI Materials Journal, 105(4), 358.

Lin, Z., Kanda, T. and Li, V. (1999). On Interface Property Characterization and Performance of Fiber Reinforced Cementitious Composites. Concrete Science and Engineering, RILEM, V(I), pp. 173- 184.

Li, M., Li, V.C. (2011). Cracking and Healing of Engineered Cementitious Composites under Chloride Environment. Mater. J. 108, 333-340.

Li, V.C. (1993). From micromechanics to structural engineering: the design of cementitious composites for civil engineering applications, Journal of Structural Mechanics and Earthquake Engineering, 10(2) 37-48.

Li, V. C. (1998). Engineered Cementitious Composites for Structural Applications. 10(2), pp. 6669.

Li, V. (2003). on Engineered Cementitious Composites (ECC) - A Review of The Material and Its Applications. Journal of Advanced Concrete Technology, 1(3), pp. 215-230.

Li, V.C. (2008). Engineered cementitious composites (ECC) - material, structural, and durability performance, in: E. Nawy (Ed.), Concrete Construction Engineering Handbook, CRC Press, p. 78.

Li, V. C. (2011). High-Ductility Concrete for Resilient Infrastructures. Journal of Advanced and High-Performance Materials, pp. 16-21.

Li, V. C. (2019). Engineered Cementitious Composites (ECC): Bendable Concrete for Sustainable and Resilient Infrastructure. Springer. 
Li, V.C. and Lepech, M. (2004). Crack resistant concrete material for transportation construction. In TRB 83rd Annual Meeting, Washington, D.C., Compendium of Papers CD ROM, Paper 04-4680.

Li, V. and Stang, H. (2004). Elevating FRC Material Ductility to Infrastructure Durability. Varenna, Lake Como, Italy, Proceedings of BEFIB, pp. 171-186.

Li, V.C., Fischer, G., Kim, Y.Y., Lepech, M., Qian, S., Weimann, M., and Wang, S. (2003). Durable Link Slabs For Jointless Bridge Decks Based on Strain-Hardening Cementitious Composites: Report for Michigan Department of Transportation RC- 1438.

Li, V.C., Horikoshi, T., Ogawa, A., Torigoe, S., and Saito, T. (2004). Micromechanics-based Durability Study of Polyvinyl Alcohol Engineered Cementitious Composite (PVA-ECC). ACI Materials Journal, 101(3), pp. 242-248.

Li Victor C., Leung Christopher K. Y. (1992). Steady-State and Multiple Cracking of Short Random Fiber Composites. J. Eng. Mech. 118, 2246-2264.

Li, V. C., Wang, S. and Wu, C. (2001). Tensile Strain Hardening Behaviour of PVAECC. ACI Material Journal, 98(6), pp. 483-492.

Manning, D. (1996). Corrosion Performance of Epoxy-Coated Reinforcing Steel: North American Experience. Construction and Building Materials, 10(5), pp. 349-365.

Martys, N. and Ferraris, C. (1997). Capillary Transport In Mortars and Concrete. Cement and Concrete Research, 27(5), pp. 747-760.

Maruta, M., Kanda, T., Nagai, S. and Yamamoto, Y. (2005). New high-rise RC Structure Using Pre-Cast ECC Coupling Beam. Concrete Journal, 43(11), pp. 18-26.

Mavani, M. B. (2012). Fresh/mechanical/durability properties and structural performance of engineered cementitious composite (ECC). MASc Thesis, Ryerson University, Toronto, Canada.

Mehta, P. (1986). Concrete: Structure, Properties, and Materials. Englewood Cliffs New Jersey: Prentice-Hall.

Mihashi, H. and De Leite, J. (2004). State-of-the-Art Report on Control of Cracking in Early Age Concrete. Advanced Concrete Technology, 2(2), pp. 141-154.

Mitamura, H., Sakata, N., Shakushiro, K., Suda, K. and Hiraishi, T. (2005). Application of overlay reinforcement method on steel deck utilizing engineering cementitious composites - Mihara Bridge. Bridge and Foundation Engineering 39(8):88-91.

Miyazato, S. and Hiraishi, Y. (2005). Transport Properties and Steel Corrosion In Ductile Fiber Reinforced Cement Composites. Turin, Italy, Proceedings of the Eleventh International Conference on Fracture, pp. 20-25.

Naaman, A. E. (1992). SIFCON: tailored properties for structural performance. In H. W. Reinhardt, \& A. E. Naaman (Eds.), High-performance fiber-reinforced cement composites, (pp. 18-38). E \& FN Spon,

Nawy, E. G. (2008). Concrete construction engineering handbook. CRC Press, Boca Raton, FL. 
Nikopour, H., \& Nehdi, M. (2011). Shear repair of RC beams using epoxy injection and hybrid external FRP. Materials and Structures/Materiaux et Constructions, 44(10), 1865-1877.

Oh, B.H., and Shin, K.J. (2006). Cracking, ductility and durability characteristics of HPFRCC with various mixture proportions and fibers, in Proc., Int'l RILEM Workshop on High Performance Fiber Reinforced Cementitious Composites in Structural Application. Eds. Fischer, G., and V.C. Li, Eds. Fischer, G., and V.C. Li, published by RILEM SARL, pp. 213222.

Özbay, E. Karahan, O. Lachemi, M. Hossain, K.M.A. and Atis, C.D. (2012). Investigation of Properties of Engineered Cementitious Composites Incorporating High Volumes of Fly Ash and Metakaolin. ACI materials journal, 109(5), pp. 565-571.

Özbay, E. Karahan, O. Lachemi, M. Hossain, K. M. A. and Atis C. D. (2013). Dual Effectiveness of Freezing-Thawing and Sulfate Attack on High-Volume Slag-Incorporated ECC. Composites Part B: Engineering, 45(1), pp. 1384-1390.

Qian, S., Lepech, M. D., Kim, Y. Y. and Li, V. C. (2009). Introduction of Transition Zone Design for Bridge Deck Link Slabs Using Ductile Concrete. ACI Stuctural Journal, 106(1), pp. 96105.

Rafiei S. (2011). Behaviour of double skin profiled composite shear wall system under inplane monotonic, cyclic and impact loadings. (PhD Thesis) Toronto, Canada: Department of Civil Engineering, Ryerson University

Rashed A., Rogowsky David M., Elwi A. E. (2000). Tests on Reinforced Partially Prestressed Concrete Tank Walls. J. Struct. Eng. 126, 675-683.

Reinhardt, H.-W., Jooss, M. (2003). Permeability and self-healing of cracked concrete as a function of temperature and crack width. Cem. Concr. Res. 33, 981-985.

Rokugo, K., Kunieda, M., Lim, S.C. (2005). Patching repair with ECC on cracked concrete surface. Proc. CONMAT 5.

Sadjadi, R., Ziari, A., \& Kianoush, M. R. (2010). Experimental study of RC rectangular liquid containing structures retrofitted with GFRP composites. Applied Composite Materials, 17(2), 195-207.

Sagues, A., Powers, R. and Locke, C. (1994). Corrosion Processes and Field Performance of Epoxy-Coated Reinforcing Steel In Marine Structures. Houston, TX: Corossion 94.

Şahmaran, M.L, Keskin, S.B., Ozerkan, G., Yaman, I.O. (2008). Self-healing of mechanically loaded self-consolidating concretes with high volumes of fly ash. Cem. Concr. Compos. 30, 872879.

Şahmaran, M.L, Lachemi, M., Hossain, K. M. A., Ranade, R., \& Li, V. C. (2009). Influence of aggregate type and size on ductility and mechanical properties of engineered cementitious. ACI Materials Journal, 106(3), 308-316.

Sahmaran, M.L, Li, V. and Li, M. (2007). Transport Properties of Engineered Cementitious Composites under Chloride Exposure. 104(6), pp. 604-611. 
Sahmaran, M.L and Li, V. (2007). De-Icing Salt Scaling Resistance of Mechanically Loaded Engineered Cementitious Composites. Cement and Concrete Research, Issue 37, pp. 10351046.

Sahmaran, M.L, Li, V. and Andrade, C. (2008). Corrosion Resistance Performance of SteelReinforced Engineered Cementitious Composite Beams. ACI Material Journal, 105(3), pp. 243-250.

Sahmaran, M.L and Li, V. (2008). Durability of mechanically loaded Engineered Cementitious Composites under high alkaline environment. Cement and Concrete Composites, 30(2), pp. 72- 81 .

Şahmaran, M.L., and Li, V. C. (2009a), "Durability properties of micro-cracked ECC containing high volumes fly ash", Cement and Concrete Research, 39(11) 1033-1043.

Sahmaran, M.L and Li, V. (2009b). Influence of Microcracking on Water Absorption and Sorptivity of ECC. 42(5), pp. 593-603.

Şahmaran, M.L and Li, V.C. (2009c), "Engineered cementitious composites: An innovative concrete for durable structure," Proceedings of the 2009 Structures Congress - Don't Mess with Structural Engineers: Expanding Our Role, pp. 2219-2231.

Sahmaran, M.L and Li, V. C. (2010). Engineered Cementitious Composites Can Composites Be Accepted as Crack-Free Concrete? Journal of the Transportation Research Board, No.2164, pp. 1-8.

Şahmaran, M.L., Özbay,O., Yüce, H.E., Lachemi, M., and Li, V.C. (2011), "Effect of Fly Ash and PVA Fiber on Micro-structural Damage and Residual Properties of Engineered Cementitious Composites Exposed to High Temperatures", Journal of Materials in Civil Engineering, December 2011 1735-1745.

Schlangen, E. (2010). Fracture mechanics. CT5146 Lecture Notes. In: Hua X. Self-healing of Engineered Cementitious Composites (ECC) in concrete repair system. Master Thesis Delft Univ. Technol.

Sherir, M. A. A. (2012). Fracture, fatigue and creep performance of ECC mixtures with reference bridge deck applications. MASc Thesis, Dept. of Civil Engineering, Ryerson University.

Sherir, M.A.A., Hossain, K.M.A., and Lachemi, M. (2014), Fracture Energy Characteristics of Engineered Cementitious Composites Incorporating Different Aggregates, CSCE 2014 4th International Structural Specialty Conference, Halifax, NS, May 28 to 31, 2014.

Sherir, M.A.A., Hossain, K.M.A., and Lachemi, M. (2015), Structural Performance of Polymer Fiber Reinforced Engineered Cementitious Composites Subjected to Static and Fatigue Flexural Loading, Polymers 2015, 7, 1299-1330.

Shriram, J., Sreenath, S., \& Saravana Raja Mohan, K. (2018). Strengthening of reinforced concrete beams using engineered cementitious composites. International Journal of Civil Engineering and Technology, 9(4), 608-613. 
Singh, S. B., \& Sivasubramanian, M. V. R. (2013). Flexural response of ECC strengthened reinforced concrete beams. Indian Concrete Journal, 87(7), 35-44.

Torabi, A., \& Maheri, M. R. (2017). Seismic repair and retrofit of RC beam-column joints using stiffened steel plates. Iranian Journal of Science and Technology - Transactions of Civil Engineering, 41(1), 13-26.

Thanoon, W. A., Jaafar, M. S., Kadir, M. R. A., \& Noorzaei, J. (2005). Repair and structural performance of initially cracked reinforced concrete slabs. Construction and Building Materials, 19(8), 595-603.

Wang, S., and Li, V.C. (2007), "Engineered Cementitious Composites with High-Volume Fly Ash", ACI Materials Journal, 104(3) 233-241.

Wang, G., Yang, C., Pan, Y., Zhu, F., Jin, K., Li, K., \& Nanni, A. (2019). Shear behaviors of RC beams externally strengthened with engineered cementitious composite layers. Materials, 12(13).

Wang, W., \& Zheng, Y. (2015). Flexural Strengthening Rc Beams Using a Composite Reinforcement. The 12th International Symposium on Fiber Reinforced Polymers for Reinforced Concrete Structures (FRPRCS-12), (December), 14-16.

Weimann, M. and Li, V. (2003). Hygral Behaviour of Engineered Cementitious Composites (ECC). International Journal for Restoration of Buildings and Monuments, 9, 513-534., 9(5), pp. 513-534.

Wittmann, F. (2002). Crack Formation and Fracture Energy of Normal and High Strength Concrete. Sadhana, 27(4), pp. 413-423.

Wu, M., Johannesson, B., Geiker, M. (2012). A review: Self-healing in cementitious materials and engineered cementitious composite as a self-healing material. Constr. Build. Mater. 28, 571583.

Yang, X., Gao, W. Y., Dai, J. G., Lu, Z. D., \& Yu, K. Q. (2018). Flexural strengthening of RC beams with CFRP grid-reinforced ECC matrix. Composite Structures, 189(January), 9-26.

Yang, Y., Lepech, M.D., Yang, E.-H., Li, V.C. (2009). Autogenous healing of engineered cementitious composites under wet-dry cycles. Cem. Concr. Res. 39, 382-390.

Yeganeh, A. E. (2013). Structural behavior of reinforced high-performance concrete frames subjected to monotonic lateral loading, M. Sc. Thesis, Ryerson University, Toronto, Ontario.

Yun, H. D., \& Rokugo, K. (2012). Freeze-thaw influence on the flexural properties of ductile fiber reinforced cementitious composites. Cold Regions Science and Technology, 78(1), 8288.

Zhang, Z., Zhang, Q. (2017). Self-healing ability of Engineered Cementitious Composites (ECC) under different exposure environments. Constr. Build. Mater. 156, 142-151. 
Zhu, Z., \& Wang, W. (2016). Experimental study on mechanical behaviour of circular reinforced concrete columns strengthened with FRP textile and ECC. Dongnan Daxue Xuebao (Ziran Kexue Ban)/Journal of Southeast University (Natural Science Edition), 46(5), 1082-1087.

Ziari, A., \& Kianoush, M. R. (2009). Investigation of direct tension cracking and leakage in RC elements. Engineering structures, 31(2), 466-474. 